\title{
DESIGN AND SIMULATION OF THE nuSTORM FACILITY
}

\author{
Ao Liu
}

Submitted to the faculty of the University Graduate School

in partial fulfillment of the requirements

for the degree

Doctor of Philosophy

in the Department of Physics,

Indiana University

April 2015 
Accepted by the Graduate Faculty, Indiana University, in partial fulfillment of the requirements for the degree of Doctor of Philosophy.

Doctoral Committee

\begin{tabular}{r}
\hline Shyh-yuan Lee, Ph.D., Committee Chair \\
\hline John P. Carini, Ph.D. \\
\hline Chen-yu Liu, Ph.D. \\
Mark D. Messier, Ph.D.
\end{tabular}

Fermilab advisors

Alan D. Bross, Ph.D.

$02 / 24 / 2015$

David V. Neuffer, Ph.D. 
Copyright (C) 2015

Ao Liu 
To my beloved parents and Jie 


\section{ACKNOWLEDGMENTS}

The thankful words in my mind for those who have provided invaluable help in my $\mathrm{Ph} . \mathrm{D}$. career are beyond what this page can carry. It is therefore more than necessary to generalize these words in the following short form.

Firstly, I would like to sincerely thank my supervisor and mentor, Prof. S.Y. Lee, who brought me to the world of accelerator physics and provided me with the chance to study and work at Fermilab as a Joint Ph.D program student. His useful academic advice has guided me through my Ph.D life. He is strict in physics research but very kind and easy-going in our daily life. He inspired me so much to pursue a better research career and to make my Ph.D life more meaningful and enjoyable.

In parallel, I want to offer special thanks to my supervisors at Fermilab, Dr. Alan Bross and Dr. David Neuffer, who opened the joint Ph.D. program position at Fermilab. The work with Dr. Bross and Dr. Neuffer was very enjoyable and fruitful. Without them, the idea of nuSTORM would simply not exist. They have deep understandings of both the physics of our project but also the details of the facility design. I want to thank Dr. Bross for his financial support during my stay in Fermilab to

make my office life more comfortable. His support for me to attend a broad range of conferences worldwide helped me gain better experience of presenting my work, know people in the community, and more enthusiastically put effort in research. I also want to thank Dr. Neuffer and his family for offering Christmas dinners at his house during 
these years. I specially thank Dr. Bross and Dr. Neuffer for their fatherly advices and discussions

The help from my friends is enormous. Among them I owe special thanks to my colleague and friend, Dr. David Adey, for his help in analyzing the data to produce neutrino flux from the simulation and his advices to help me express myself better in English. Also, I should not forget about the help from Ms. Margie Bruce, which enlightened the days in APC. I also would like to thank Dr. JB Lagrange, who helped me understand the goals and challenges for nuSTORM ring design better using his FFAG scenario. I sincerely thank M. Geelhoed and S. Striganov for their help in the beamline design.

I also met excellent friends in Fermilab from both the high-energy and the accelerator communities. The time spent with Dr. Ziqing Hong (quoted from his self-description: A Ping-pong fan with the best fishing skills among HEP-ex physicists), Dr. Zhen Hu (Also from his words: A Ping-pong fan with the best Gobang skills among HEP-ex physicists) and his family, Mr. Zijun Xu, Dr. Honghuan Liu, and Mr. Jeff Eldred was very pleasant and memorable.

Last but not the least, I cordially thank my family, especially my fiancée Jie Gao who gave me love, appreciation and courage to enjoy my life and work. 
Ao Liu

\section{DESIGN AND SIMULATION OF THE nuSTORM FACILITY}

This thesis provides a full design and simulation of the nuSTORM beam line facility including the pion beam line, the pion and muon orbit combination section, and the muon decay ring. The study also includes the development of numerical optimization methods and their application to the design.

At nuSTORM, one proton batch from the Main Injector (MI) is extracted and transported to the target station to bombard a solid target. A magnetic horn is used as the collection device for the secondary particles such as pions and kaons. A pion beam line is designed to transport the secondary particles downstream to the muon decay ring. In order to avoid the use of a fast kicker with a large aperture for the injection, an Orbit Combination Section (OCS) was designed to combine the reference pion orbit with the circulating muon orbit. This fulfills the stochastic injection scheme proposed in the 1980s. It is shown through the simulation that the pion beam line is able to deliver 0.011 muons per proton on target within the acceptance of the ring.

In order to improve the circulating muon flux, an optimization of the magnetic collection horn was studied. A Multi-Objective Genetic Algorithm (MOGA), that was modified to integrate Message Passing Interface (MPI) in the process, is applied to the optimization. The number of muons within the ring acceptance from the pion decay is estimated by the pions after the collection horn. This new method was proposed to lower the requirement on the computing resources for the optimization. The study

gives a new and better target and horn configuration after a full investigation of the

vii 
system.

The nuSTORM ring is examined with two optics designs. With different features, the two designs are optimized via correcting the nonlinearities with sextupolar field distribution in the ring. A modified MPI-based Simulated Annealing (SA) algorithm was applied to obtain the optimal ring acceptance. The comparison with the GA shows good agreements on the achieved optimal ring acceptance. The pion and muon beams at nuSTORM were simulated and yield flavor-pure and precisely measurable neutrino beams, which can be used to provide a definite statement about the existence of a sterile neutrino. 


\section{CONTENTS}

ACKNOWLEDGMENTS V v

ABSTRACT vii

1 Introduction to Accelerator and beam Physics 1

1.1 Curvilinear Coordinate System . . . . . . . . . . . . . 4

1.2 Transverse Motion ................... 6

1.2.1 Linear Betatron Motion . . . . . . . . . . . . . 6

1.2.2 Particle Beam . . . . . . . . . . . . . . . . 10

1.2.3 Off-momentum Orbit . . . . . . . . . . . . . . . . . 12

1.2.4 Momentum compaction factor . . . . . . . . . . . 13

1.3 Synchrotron Motion . . . . . . . . . . . . . . . . . . . . 14

2 Introduction to Neutrinos and nuSTORM 16

2.1 History of Neutrinos . . . . . . . . . . . . . . 16

2.2 Basic Neutrino Properties . . . . . . . . . . . . . . . 18

2.2.1 Neutrinos in the Standard Model . . . . . . . . . . . . . . . 18

2.2.2 Conservation of Total Lepton Number and Lepton-Family number 20

2.2.3 Neutrino Mixing . . . . . . . . . . . . . . . 21

2.2.4 CP Violation .................... . . 21

ix 
2.2 .5 Neutrino Oscillations . . . . . . . . . . . . . . . . . 22

2.2 .6 Sterile Neutrinos . . . . . . . . . . . . . . . . . 26

2.2 .7 Neutrino-Nucleon Scattering . . . . . . . . . . . . . . . 26

2.2.8 Total cross section for neutrino-nucleon scattering . . . . . 28

2.3 Introduction to nuSTORM . . . . . . . . . . . . . . . . 28

2.3 .1 Overview and Motivation . . . . . . . . . . 30

2.3.2 Introduction to the target and horn . . . . . . . . . . . 34

2.3.3 Introduction to the simulation tools . . . . . . . . . . . 37

3 Pion Beamline D\&S for nuSTORM 40

3.1 Definition of the pion beamline and stochastic injection . . . . . . 41

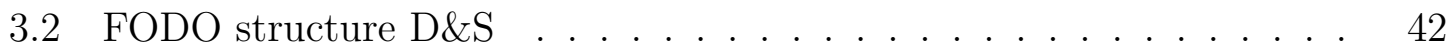

$3.2 .1 \quad$ FODO cell design strategy . . . . . . . . . . . . . 43

3.2.2 Twiss functions for the two reference momenta . . . . . . . 44

3.3 From the FODO to the OCS . . . . . . . . . . . . . . . . 45

3.3 .1 Introduction . . . . . . . . . . . . . . . 45

3.3.2 Design procedure . . . . . . . . . . . . . . . . . 48

3.4 From the OCS to the horn . . . . . . . . . . . . . . . 53

3.4 .1 Introduction . . . . . . . . . . . . . 53

3.4.2 Twiss functions of pions after the horn . . . . . . . . 55

3.4.3 Dispersion Suppression and matching to the horn . . . . . 56

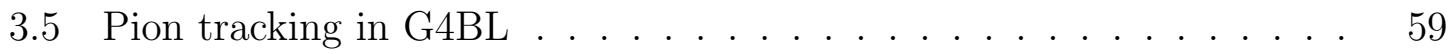

3.5.1 The muons at the end of the production straight . . . . . . 60

3.5.2 Possible contamination of the injected beam . . . . . . . . . 61

3.6 Summary . . . . . . . . . . . . . . . . . 66 
4 Optimization of the magnetic horn for nuSTORM $\quad 68$

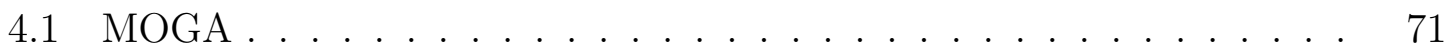

4.2 MOGA extended and modified . . . . . . . . . . . 73

4.3 Objective 1: Maximizing $N_{\mu, P} \ldots \ldots \ldots 76$

4.3.1 Decay kinematics of a single pion with momentum $p_{\pi} \ldots$. . . 76

4.3.2 Decay kinematics of a pion beam with a linear momentum dis-

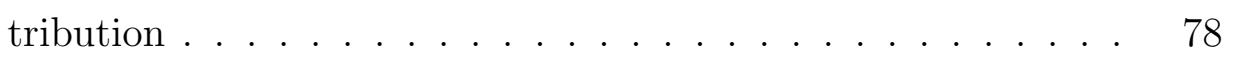

4.3.3 Estimating the the number of $\mu^{+}$in $\Omega_{P}$ at the end of the production straight . . . . . . . . . . . . . . 82

4.4 Objective 2: Maximizing $N_{\pi, \epsilon} \ldots \ldots \ldots$. . . . . . . 87

4.5 Application of pyOPTmpi . . . . . . . . . . . . . . 89

4.5.1 Algorithm flowchart . . . . . . . . . . . . . . . 89

4.5.2 Optimization results .................. 89

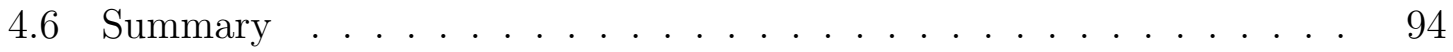

5 nuSTORM decay ring design and simulation and neutrino flux at $\begin{array}{ll}\text { nuSTORM } & 96\end{array}$

5.1 Decay Ring Design and Simulation . . . . . . . . . . . . . . 99

5.1.1 Arc design with superconducting magnets . . . . . . . . . . 100

5.1 .2 Sextupole correction optimization . . . . . . . . . . . . 102

5.1.3 Arc design with conventional, non-superconducting magnets . 116

5.2 Neutrino flux at nuSTORM . . . . . . . . . . . . . 120

5.2.1 Neutrino flux from the pion beam . . . . . . . . . . . . 122

5.2.2 Neutrino flux from the muon beam . . . . . . . . . . . . . . 122

5.3 Summary . . . . . . . . . . . . . . . . . . 124 
A.1 Gauss-Newton Algorithm . . . . . . . . . . . . . . . . . . . 130

A.1.1 Gauss-Newton in bivariate Gaussian fitting . . . . . . . . . . . 131

A.2 Simulated Binary Crossover Operator . . . . . . . . . . . . . 132

A.3 Simulated Annealing Algorithm . . . . . . . . . . . . . . . . . . . 134

A.4 Converting MAD-X outputs to G4BL input . . . . . . . . . . . 136

A.4.1 Requirements and Strategies . . . . . . . . . . . . 136

A.4.2 Pseudo-code of "mad2g4bl.py" . . . . . . . . . . . . . . 137

A.5 Definition of the MADX pole-face rotation angles . . . . . . . . 138

\section{Curriculum Vitae}




\section{LIST OF FIGURES}

1.1 The proposed nuSTORM siting at in Fermilab. . . . . . . . . . . 3

1.2 Curvilinear coordinate system for particle motion in a synchrotron. . 5

1.3 Invariant ellipse of the particle motion in the phase space. . . . . . . 10

2.1 The standard model particles. The mass for the Higgs boson was measured by CMS and ATLAS, to be $125.03_{-0.27}^{+0.26}$ (stat) ${ }_{-0.15}^{+0.13}$ (sys) and $125.36 \pm 0.37$ (stat) \pm 0.18 (sys) $\mathrm{GeV} / \mathrm{c}^{2}[5,6]$ (Courtesy of schoolswikipedia.org) . . . . . . . . . . . . . . . . . 19

2.2 The sterile neutrino search from different experiments. The results from MINOS that were presented in June, 2014 exclude everything to

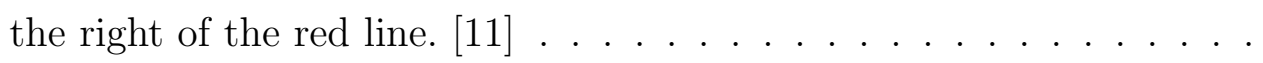

2.3 The total CC cross section for $\nu_{\mu}$ (upper) and $\bar{\nu}_{\mu}$ (lower) as a function of neutrino energy. Solid lines show the calculated total cross sections. The dashed, dot, and dash-dotted lines are the calculated quasi-elastic single-meson and deep-inelastic scatterings, respectively. [12] . . . . .

2.4 The schematic drawing and the engineering layout of the nuSTORM facility (upper and lower, respectively). . . . . . . . . . . 
2.5 The schematic drawing of the NuMI horn 1 (upper) and LBNE horn 1 (middle). Both of the horns have a parabolic Inner Conductor (IC) for the sections before and after the neck. The schematic drawing of the nuSTORM baseline target and horn (bottom) shows the actual size of these target station elements. The nuSTORM horn runs at a peak current of $230 \mathrm{kA} \ldots \ldots \ldots \ldots \ldots \ldots$

2.6 The phase space distribution of pions at the downstream end of the nuSTORM baseline horn. The simulation was done in MARS15 [20] and the numbers correspond to $2.4 \times 10^{7} 120 \mathrm{GeV}$ POT. The meaning of the green and red ellipses will be explained later in Section 3.4. . .

3.1 The schematic drawing of a FODO cell (upper) and the model in G4BL (lower). The quadrupoles are in red and the drift spaces are in white in the G4BL drawing. . . . . . . . . . . . . . . . .

3.2 The absolute values of the three criteria. The smaller values are always represented in blue and the larger values are in red. The number of muons in $3.8 \pm 10 \% \mathrm{GeV} / \mathrm{c}$ (upper left) and $1 / \sigma_{\theta}^{2}$ (upper right) are to be maximized, while the number of cells is to be minimized (lower). The three criteria favor (lower $\beta_{F}$, lower $\beta_{D}$ ), (higher $\beta_{F}$, higher $\beta_{D}$ ), (higher $\beta_{F}$, lower $\beta_{D}$ ), respectively. . . . . . . . . . . . . . . . . 46 
3.3 The periodic Twiss parameters for the two reference particles in the 5 nuSTORM production straight FODO cells (upper left - $3.8 \mathrm{GeV} / \mathrm{c}$ muon; upper right - $5 \mathrm{GeV} / \mathrm{c}$ pion). The setup of the FODO cell is the same for both left and right. As a comparison, the Twiss parameters for $5 \mathrm{GeV} / \mathrm{c}$ pion in the 5 cells starting from the same values with the muon is shown in the lower plot. The $\beta_{u}(u=x, y)$ quickly jumps to very large values that cause an excessively large beam size. . . . . . . 47

3.4 The schematic drawing of the OCS. The beams move left-to-right. 49

3.5 The optics of the $3.8 \mathrm{GeV} / \mathrm{c}$ reference muon from the FODO structure to the injection point. Both the injected pions and circulating muons move right-to-left. $\beta_{x}$ : black, $\beta_{y}$ : red, $D_{x}$ : green. . . . . . . . . . .

3.6 The schematic drawing of the two orbits from the downstream end of the dipole to the injection point. The specific points of interest are marked in red, where the pion orbit is tracked and recorded to obtain the correct optics. . . . . . . . . . . . . . . . .

3.7 The corresponding reference pion optics from the FODO structure to the injection point. The magnets have been changed in MADX to match the $5 \mathrm{GeV} / \mathrm{c}$ pion's reference orbit. $\beta_{x}$ : black, $\beta_{y}$ : red, $D_{x}$ : green. 54

3.8 The IGN flowchart of the Iterative Gauss-Newton (IGN) fitting algorithm. An example of its application to fitting the transverse phase space distribution at the downstream end of the NuMI-like nuSTORM baseline horn is shown in Figure 2.6. The IGN algorithm (green) can better preserve the core of the phase space than the fitting using a covariance matrix method. . . . . . . . . . . . . . . 
3.9 The pion beamline optics plotted in the pion beam direction, from the downstream end of the horn to the start of the FODO structure. The location of OCS is highlighted by the red block. The entire production straight section is represented by a single FODO cell to avoid repetitive plotting of the Twiss functions. The pions move from the left to the right in the drawing. $\beta_{x}$ : black, $\beta_{y}$ : red, $D_{x}:$ green. . . . . . . . . . 58

3.10 A screenshot of the pion beamline structure in G4BL display mode. The white, red and yellow blocks correspond to drift spaces, quadrupoles, and sector dipoles, respectively. The beam moves from the left to the right. . . . . . . . . . . . . . . . . .

3.11 The X-X' phase space, X-Y real space and momentum distribution of the pions after the "simple" nuSTORM baseline horn with a $38 \mathrm{~cm}$ Inconel target. The data corresponds to $2.4 \times 10^{7} 120 \mathrm{GeV}$ POT. The pion yield in $5 \pm 1 \mathrm{GeV} / \mathrm{c}$ and $r \leq 15 \mathrm{~cm}$ is 0.29 per POT, with no cuts on the particle actions $J_{i} \ldots \ldots \ldots \ldots$

3.12 The phase space (upper left), real space (upper right) and momentum (lower) distributions of muons at the end of the production straight. The data corresponds to $2.4 \times 10^{7} 120 \mathrm{GeV}$ POT. The green $P$ band $(3.8 \pm 10 \% \mathrm{GeV} / \mathrm{c})$ is the momentum acceptance range of the decay ring, and the red band $(5 \pm 10 \% \mathrm{GeV} / \mathrm{c})$ is the OCS mirror extraction momentum range. . . . . . . . . . . . . . . . . .

3.13 The X-X' phase space, $\mathrm{X}-\mathrm{Y}$ real space and momentum distributions of the pions after a "real" nuSTORM baseline horn with a $38 \mathrm{~cm}$ Inconel target. The data correspond to $2.4 \times 10^{7} 120 \mathrm{GeV}$ POT. The pion yield in $5 \pm 1 \mathrm{GeV} / \mathrm{c}$ and $r \leq 15 \mathrm{~cm}$ is 0.32 per POT. . . . . . . . . 
4.1 Upper: The transverse phase space histogram of the pions produced by bombarding a $38 \mathrm{~cm}$ (left) and a $46 \mathrm{~cm}$ (right) Inconel target with 120 $\mathrm{GeV}$ protons, simulated by MARS. The data corresponds to $2.4 \times 10^{7}$ Protons On Target (POT). Lower: the histogram of longitudinal positions where the pions are emitted from a $38 \mathrm{~cm}$ target. . . . . . . . .

4.2 The crowding distance that estimates the density of the population surrounding one particular individual $i$ by measuring the average side length of the cuboid formed by its nearest neighbors. $f_{k}$ and $f_{k+1}$ are two objectives. In this figure, individual $i$ has larger crowding distance than $j \ldots \ldots \ldots \ldots \ldots \ldots \ldots \ldots \ldots$

4.3 The momentum distribution of pions at the downstream face of an example horn, simulated by MARS15. The fitted probability density function and the fitting are shown in red. . . . . . . . . . .

4.4 Comparison of the density function $f_{\mathbf{p}}(p)$ obtained from the analytical formula in Equation (4.13) (blue solid line) and sampling the MC decay data in G4BL (gray bar plot), using a pion beam with $m=0.1, P_{0}=5$ $\mathrm{GeV} / \mathrm{c}$. The red and black curves are obtained by Equation(4.13) for pion beams with $m=0.164$ and $m=0.2$, respectively. $N_{0}$ and $P_{0}$ are the same for all curves. The $\Omega_{P}$ range is marked in green. . . . . . . .

4.5 The transmission efficiency of $\pi^{+}$in the pion beamline with different momentum spreads. The tracking results from numerically generated Gaussian pion beams with different $m$ are plotted with red triangles. The function fitted to those results is plotted with the black solid line. The tracking results from the MARS simulated pion beam after the horn is plotted in green rhombuses. . . . . . . . . . . . . 
4.6 Comparison of $N_{\mu, P}(m)$ (formula) and the results from G4Beamline tracking of the pion beam from an Inconel target and the horn (red) and with the target changed to Graphite (green). The numbers are scaled to compare the normalized production rate. . . . . . . . .

4.7 The feasible range of Twiss parameters at the downstream end of the horn that can be matched by conventional capture quads. The fitness of horns that yield Twiss parameters outside the range and their priorities in making the offspring are evaluated using the distance to the boundary of the feasible range. . . . . . . . . . . . . .

4.8 Schematic drawing of the horn showing the parameters that form the genes in the MOGA for this horn optimization study. Eight of the nine are shown by the symbols $\mathrm{L} 1, \ldots, \Delta \mathrm{Z}$, and the ninth is the horn current $I .90$

4.9 The flowchart for the MOGA applied to the horn optimization. The loop that has the MPI implemented is marked with the blue circle. . 91

4.10 The evolution of the objective values for the two searches for optimum horn configurations with $38 \mathrm{~cm}$ (above) and $46 \mathrm{~cm}$ (below) Inconel targets. The arrows are formed when the best individual in an older generation is replaced by another in a newer generation. . . . . . .

4.11 A comparison of the phase space distributions of pions focused by the pre-optimized and the optimized horn (top and bottom, respectively). The red ellipses are the fitted Gaussian acceptance ellipses. The number of pions included in the acceptance is $6.9 \times 10^{5}$ and $8.2 \times 10^{5}$ (a $19 \%$ increase) from $2.4 \times 10^{6} 120 \mathrm{GeV}$ POT, respectively. For reference, $1.2 \times 10^{6}$ pions emitted from the target surface, respectively. . . . . . . 
4.12 The shape of the pre-optimized horn (left) and the optimized horn (right) for a $46 \mathrm{~cm}$ Inconel target. The algorithm stopped at the $81^{\text {st }}$ generation. The effective rear boundary of the horn conductor is shown with the dashed line considering that only forward pions are useful. The optimized horn has a simpler configuration, which can reduce the maintenance and the operation costs. The optimized current is reduced from $230 \mathrm{KA}$ to $\sim 219 \mathrm{kA} . \ldots \ldots \ldots$

5.1 The linear optics of a nuSTORM decay ring design with superconducting combined function dipoles in the arcs. $\beta_{x}$ : black, $\beta_{y}$ : red, $D_{x}$ : green. . . . . . . . . . . . . . . . . . . . 101

5.2 The Frequency Map Analysis (FMA) of the ring design with combined function dipoles in the arcs in the $\mathrm{X}-\mathrm{Y}$ space (left) and on the betatron tune plane (right), on-momentum particles (upper) and off-momentum particles within the full range of $\delta= \pm 0.1$ (lower) are analyzed. No sextupole corrections added. . . . . . . . . . . . . . . . . . 103

5.3 The FMA of the post-sextupole optimization ring design with combined function dipoles in the arcs in the $\mathrm{X}-\mathrm{Y}$ space (left) and on the betatron tune plane (right), on-momentum particles (upper) and offmomentum particles within the full range of $\delta= \pm 0.1$ (lower) are analyzed. . . . . . . . . . . . . . . . . 106 
5.4 The average number of turns that particles in each momentum bin survive. The data were obtained from the tracking of $10^{5}$ particles, with $2 \mathrm{~mm} \cdot \mathrm{rad}$ transverse emittance and a momentum spread of $\delta \in$ $\pm 10 \%$. The upper, middle, and lower plots correspond to data from the pre-optimized lattice, post-optimized lattice, and their difference (post minus pre) . . . . . . . . . . . . . . . . . . . 107

5.5 The average number of turns that particles survive for each $\epsilon_{x}$ bin. The data were obtained from tracking of $10^{5}$ particles, with $2 \mathrm{~mm} \cdot \mathrm{rad}$ transverse admittance and a momentum spread of $\delta \in \pm 10 \%$. The upper, middle, and lower plots correspond to data from the pre-optimized lattice, post-optimized lattice, and their difference (post minus pre). . 109

5.6 The effectiveness of the sextupole correction optimization. Upper: the acceptance of different initial beams without optimization; Lower: the acceptance of different initial beams with an optimized lattice. . . . . 110

5.7 The increase in the average number of turns that particles survive for each momentum and $\epsilon_{x}$ bin. The data were obtained from tracking of $10^{5}$ particles, with $1 \mathrm{~mm} \cdot \mathrm{rad}$ transverse admittance and a momentum

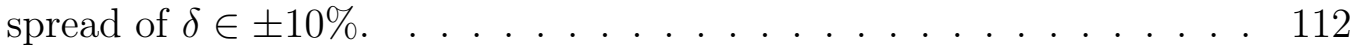

5.8 The increase in acceptance of different beams, represented by the ratio of the acceptance of the optimized lattices to the linear lattice. Upper: the original optimized lattice; Lower: the optimized lattice for a beam with $1 \mathrm{~mm} \cdot \mathrm{rad}$ transverse admittance and a momentum spread of $\delta \in$ $\pm 10 \% \ldots \ldots \ldots \ldots \ldots \ldots \ldots \ldots \ldots$ 
5.9 The increase in the average number of turns that particles survive for each momentum and $\epsilon_{x}$ bin. The data were obtained from tracking of $10^{5}$ particles, with $2 \mathrm{~mm} \cdot \mathrm{rad}$ transverse admittance and a momentum spread of $\delta \in \pm 5 \% \ldots \ldots \ldots \ldots \ldots \ldots$

5.10 The increase in acceptance of different beams, represented by the ratio of the acceptance of the optimized lattices to the linear lattice. Upper: the original optimized lattice; Lower: the optimized lattice for a beam with $2 \mathrm{~mm} \cdot \mathrm{rad}$ transverse admittance and a momentum spread of $\delta \in$ $\pm 5 \% \ldots \ldots \ldots \ldots \ldots \ldots \ldots \ldots$

5.11 The average number of turns that particles in each momentum bin survive. The data were obtained from tracking of $10^{5}$ particles, with 2 $\mathrm{mm} \cdot \mathrm{rad}$ transverse emittance and a momentum spread of $\delta \in[0.02,0.04]$. The upper, middle, and lower plots correspond to data from the preoptimized lattice, post-optimized lattice, and their difference (postminus pre-). . . . . . . . . . . . . . . . . 11

5.12 The increase in acceptance of different beams, represented by the ratio of the acceptance of the optimized lattices to the linear lattice. Upper: the original optimized lattice; Lower: the optimized lattice for a beam with $2 \mathrm{~mm} \cdot \mathrm{rad}$ transverse admittance and a momentum spread of $\delta \in$ $[0.02,0.04] \ldots \ldots \ldots \ldots \ldots \ldots \ldots$

5.13 The linear optics of a nuSTORM decay ring design with conventional combined function dipoles in the arcs. $\beta_{x}$ : black, $\beta_{y}$ : red, $D_{x}$ : green. . 119 
5.14 The average number of turns that particles in each momentum bin survive. The data were obtained from tracking of $10^{5}$ particles, with 2 $\mathrm{mm} \cdot \mathrm{rad}$ transverse emittance and a momentum spread of $\delta \in \pm 10 \%$. The upper, middle, and lower plots correspond to data from the preoptimized lattice, post-optimized lattice, and their difference (post minus pre). . . . . . . . . . . . . . . . . . . 121

5.15 Neutrino flux from the sampled injection beam of all particle types, normalized to $10^{21} 120 \mathrm{GeV}$ POT. The top and bottom plots correspond to the flux at the near $(50 \mathrm{~m})$ detector and far $(2000 \mathrm{~m})$ detector, respectively. . . . . . . . . . . . . . . . . . . . 123

5.16 Neutrino flux from the sampled muon beam along the production straight, normalized to $10^{21} 120 \mathrm{GeV}$ POT. The top and bottom plots correspond to the flux at the near detector and far detector, respectively for the beam in the first $1.6 \ldots$. . . . . . . . . . . . . . 126

A.1 Distribution of offspring values from two parents 2 and 3 with different $\eta_{c}$. The child generation is closer to the parents with greater $\eta_{c}$. . . 133

A.2 Examples of bending magnets with (in solid and blue) and without (in dashed and black) pole-face rotation angles. The upper and lower figures represent the SBEND and RBEND configurations, respectively. 140 


\section{CHAPTER 1}

\section{INTRODUCTION TO ACCELERATOR AND BEAM PHYSICS}

Since the atomic nucleus was discovered by Rutherford in 1911, there has been tremendous development in modern particle accelerators and their wide spread application in science and industry. The development of particle storage rings and circular colliders has provided particle beams of high intensity, which can be used in collisions to study the physics of elementary particles, for studying the properties of materials, and even for handling nuclear wastes. Besides the conventionally accelerated and stored particles like electrons, protons, and heavy ions, the benefit of studying muon accelerators and muon storage rings was motivated by their applications in particle physics. Among the muon accelerator facilities that have been investigated, a muon storage ring that can produce neutrino beams with well known flux and flavor has been studied. This so-called "neutrino factory" is a design concept based on muon storage rings.

A neutrino factory consists of a target, a buncher, a phase rotator, a cooling device, $\mathrm{RF}$ acceleration and storage rings that result in a clean, high intensity stored muon beam. The R\&D challenges and cost of such a facility are still being actively investigated $[1,2]$.

The neutrinos from STORed Muons (nuSTORM) physics program uses a muon 
storage ring to provide neutrino beams for multiple purposes. It can serve future longbaseline neutrino-oscillation programs by providing definitive (percent-level precision) measurements of ${ }^{\complement} \vec{\nu}_{e}{ }_{e} N$ and ${ }^{\complement} \vec{\nu}_{\mu}{ }_{\mu} N$ (interactions of neutrinos and anti-neutrinos with hadrons) scattering cross sections over a wide ( 0.5 to $\sim 4 \mathrm{GeV}$ ) neutrino energy range. It can also search for the existence of sterile neutrinos [3]. It can provide a testbed for future muon facilities, such as the full-scale neutrino factories, 6D muon cooling and the muon collider. It relies on well-demonstrated technology and can be built with components that are technically ready at the time of this thesis. In particular, the nuSTORM facility excludes two of the most expensive parts in a full-scale neutrino factory: the cooling and RF devices.

The current design of the nuSTORM facility is composed of a primary proton extraction beamline from the Fermilab Main Injector (MI), a target station, a pion beamline, a muon storage ring that shares the production straight section with the pion beamline, and the neutrino detectors. The pion beamline (see Chapter 3) is the section of the facility within which the pion beam is transported. The schematic drawing of the proposed facility sited at Fermilab is shown in Figure 1.1.

The design and simulation work on the nuSTORM beamline described in this thesis provides understanding of the beam dynamics, the requirements on instrumentation, and an evaluation of the neutrino flux provided by this facility. The Design and Simulation (D\&S) of nuSTORM is a combination of the beamline lattice design, the multi-particle tracking in magnetic fields, with the Monte Carlo (MC) simulation of stochastic processes such as particle interactions with matter and particle decay. In this thesis, the criteria and strategies for the nuSTORM D\&S are described. Since there is no existing facility that is similar to nuSTORM, the understanding of such a beamline as a neutrino source is the central goal of this thesis. Therefore, opti- 


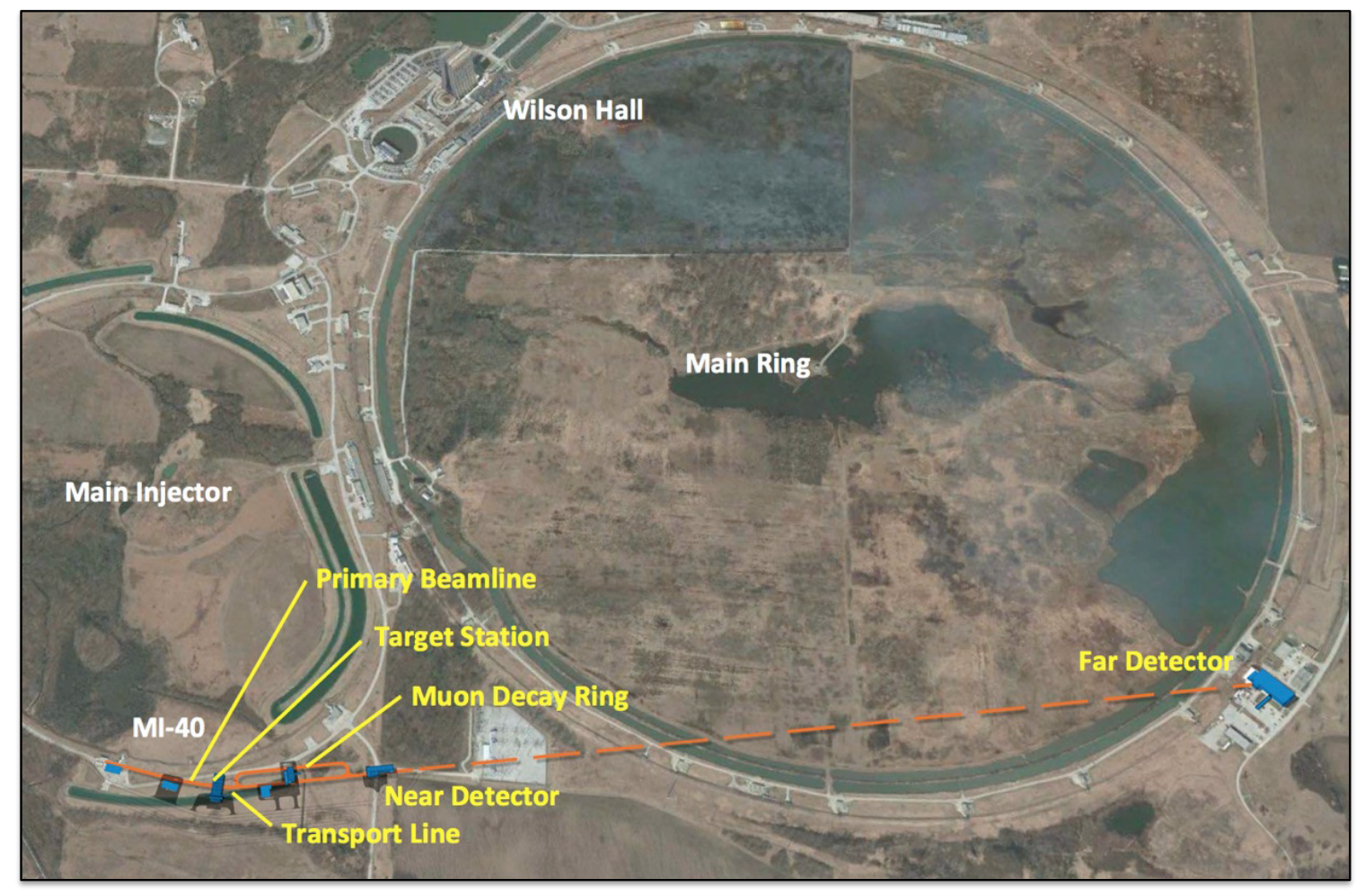

Figure 1.1: The proposed nuSTORM siting at in Fermilab. 
mization of the beamline elements is critical to understand the potential of such a facility. The optimization algorithms and strategies are discussed in this thesis. The thesis is a summary of the work conducted on the nuSTORM beamline D\&S and a record of the most recent results. The basic concepts of accelerator physics, including the curvilinear coordinate system, linear betatron motion, nonlinear effects and chromatic aberrations, and synchrotron motion are introduced in Chapter 1 (this chapter). Chapter 2 gives an introduction to the basics of neutrino physics, to the physics motivation for nuSTORM and gives an overview of its facilities. Chapter 3 describes the detailed design of the pion beamline and demonstrates its capability of delivering an intense pion beam from the target to the ring. Chapter 4 will be dedicated to the development of a new numerical method to optimize the nuSTORM magnetic horn. Chapter 5 describes the nuSTORM muon decay ring design, and the neutrino beams at nuSTORM.

In this chapter, the basic accelerator and beam physics will be introduced, which will serve as the basis for the design work hereafter. The most important concepts will be summarized, but the detailed derivations will be left out. Accelerator physics is an established branch of physics and it includes many topics and has tight connections to other fields in physics. It is a composite application of theoretical mechanics, electrodynamics, special relativity, electrical and mechanical engineering.

\subsection{Curvilinear Coordinate System}

An accelerator beamline design starts with a reference particle, which forms a theoretical trajectory through the center of a series of magnets along its path. In a circular accelerator, namely an accelerator ring, this theoretical trajectory is closed

and is referred to as the reference orbit, or the design orbit. It is the orbit that the 


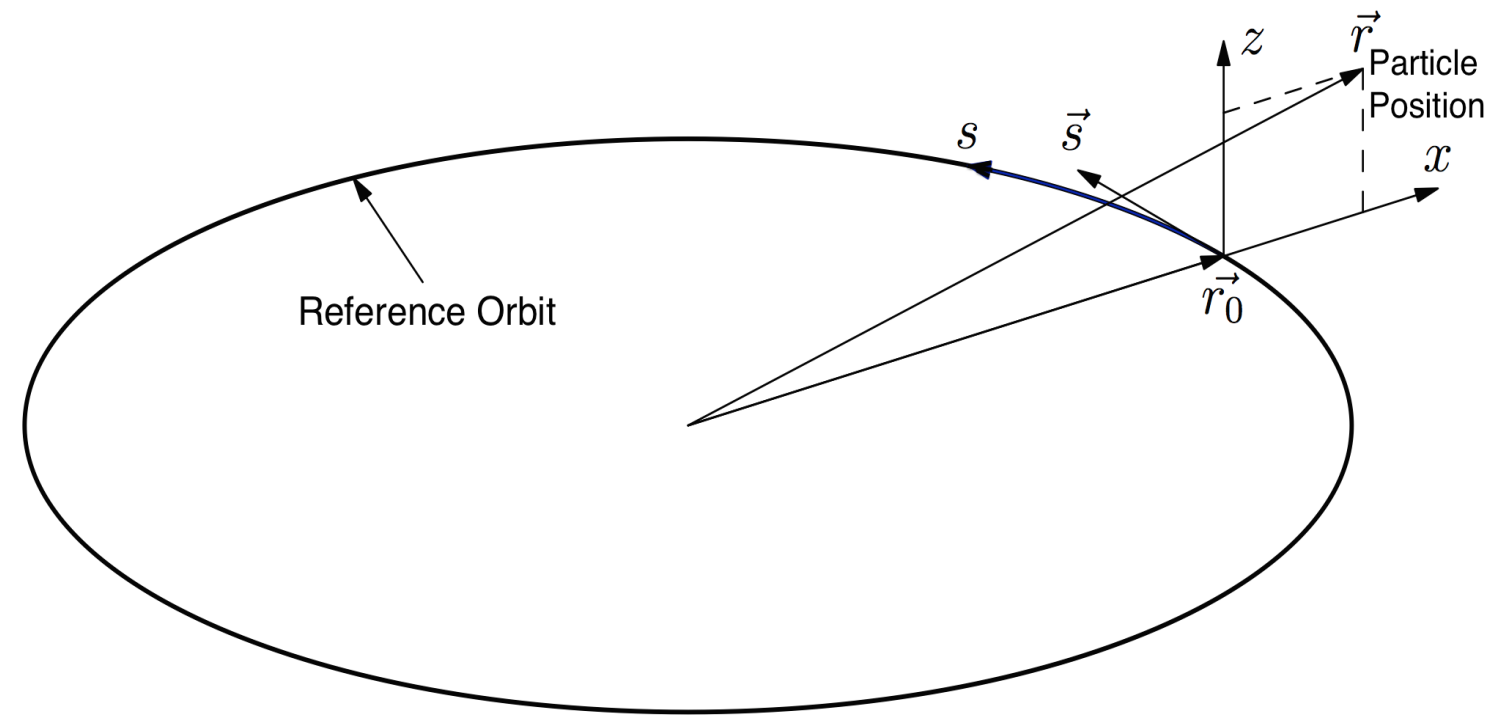

Figure 1.2: Curvilinear coordinate system for particle motion in a synchrotron.

reference particle follows under ideal conditions. However, real particles normally start their motion with small deviations from the reference orbit, which eventually result in oscillations around the reference orbit. Instead of Cartesian or cylindrical coordinate systems, the oscillations can be more conveniently described by a curvilinear coordinate system.

Let $\mathbf{r}_{0}(s)$ be the reference orbit on a plane, and $s$ measured along the orbit from a certain starting point. The coordinate system in Figure 1.2 can be described using the unit vectors as follows

$$
\hat{\mathbf{s}}(s)=\frac{d \mathbf{r}_{0}(s)}{d s}, \quad \hat{\mathbf{x}}(s)=-\rho(s) \frac{d \hat{\mathbf{s}}(s)}{d s}, \quad \hat{\mathbf{z}}(s)=\hat{\mathbf{x}}(s) \times \hat{\mathbf{s}}(s)
$$

where $\rho(s)$ defines the radius of the local curvature. The position of a single particle can be written as

$$
\mathbf{r}(s)=\mathbf{r}_{\mathbf{0}}(s)+x \hat{\mathbf{x}}+z \hat{\mathbf{z}}
$$


Instead of time $t, s$ can be used as the independent variable in the system. The particle motion can then be described by a new Hamiltonian

$$
\tilde{H}=-\left(1+\frac{x}{\rho}\right)\left[\frac{(H-e \Phi)^{2}}{c^{2}}-m^{2} c^{2}-\left(p_{x}-e A_{x}\right)^{2}-\left(p_{z}-e A_{z}\right)^{2}\right]^{1 / 2}-e A_{s}
$$

where $\mathbf{A}\left(A_{x}, A_{z}, A_{s}\right)$ is the vector potential of the magnetic field, $p_{x}, p_{z}, p_{s}$ are the conjugate momenta of coordinates $(x, z, s)$, and $\Phi$ is the electrostatic potential. For transverse magnetic fields in an accelerator, $A_{x}=A_{z}=0$, and the two-dimensional magnetic field can be expressed as $\mathbf{B}=B_{x}(x, z) \hat{x}+B_{z}(x, z) \hat{z}=-\frac{1}{h_{s}} \frac{\partial A_{s}}{\partial z} \hat{x}+\frac{1}{h_{s}} \frac{\partial A_{s}}{\partial x} \hat{z}$ where $h_{s}$ is the scale factor for the Frenet-Serret coordinate system, $h_{s}=1+\frac{x}{\rho}$. Remembering that $p=\sqrt{E^{2} / c^{2}-m^{2} c^{2}}$, the above Hamiltonian can be expanded to second order in $p_{x}$ and $p_{z}$, assuming that the transverse momenta $p_{x}$ and $p_{z}$ are much smaller than $p$,

$$
\tilde{H} \approx-p\left(1+\frac{x}{\rho}\right)+\frac{1+x / \rho}{2 p}\left[\left(p_{x}-e A_{x}\right)^{2}-\left(p_{z}-e A_{z}\right)^{2}\right]-e A_{s}
$$

\subsection{Transverse Motion}

\subsubsection{Linear Betatron Motion}

The transverse motion of a single particle in a synchrotron is the combination of its

closed orbit and a small-amplitude betatron oscillation. The closed orbit is a complete revolution of the particle beam guided by an arrangement of dipoles. The betatron motion around the closed orbit is determined by an arrangement of quadrupoles, which define the accelerator lattice.

Considering the Hamilton's equations of motion,

$$
x^{\prime}=\frac{\partial \tilde{H}}{\partial p_{x}}, \quad p_{x}^{\prime}=-\frac{\partial \tilde{H}}{\partial x}, \quad z^{\prime}=\frac{\partial \tilde{H}}{\partial p_{z}}, \quad p_{z}^{\prime}=-\frac{\partial \tilde{H}}{\partial z}
$$


and Equation 1.4, the betatron equations of motion are written as follows,

$$
x^{\prime \prime}-\frac{\rho+x}{\rho^{2}}= \pm \frac{B_{z}}{B \rho} \frac{p_{0}}{p}\left(1+\frac{x}{\rho}\right)^{2}, \quad z^{\prime \prime}=\mp \frac{B_{x}}{B \rho} \frac{p_{0}}{p}\left(1+\frac{x}{\rho}\right)^{2}
$$

With an expansion of the magnetic field up to first order in $x$ and $z, B_{z}=\mp B_{0}+B_{1} x$, $B_{x}=B_{1} z$ where $B_{1}=\frac{\partial B_{z}}{\partial x}$, the betatron equations of motion for an on-momentum particle with $p=p_{0}$ become,

$$
x^{\prime \prime}+K_{x}(s)=0, \quad z^{\prime \prime}+K_{z}(s) z=0
$$

where $K_{x}(s)=1 / \rho^{2} \mp K_{1}(s) / B \rho$ and $K_{z}(s)= \pm K_{1}(s) . K_{1}(s)=B_{1}(s) / B \rho$ is the effective focusing function and $B \rho=p / q$ is the magnet rigidity. $p$ and $q$ are the momentum and charge of the particle. The upper and lower signs correspond to positive and negatively charged particles, respectively. For example, for a pure quadrupole, $K_{x}=-K_{z}$, while for a pure sector dipole that bends the beam solely in the $x$ direction, $K_{x}=1 / \rho^{2}$ and $K_{z}=0$.

Normally, the focusing functions are arranged so that they satisfy the periodic condition $K_{y}(s+L)=K_{y}(s)$, where $y$ stands for either $x$ or $z$. The general solution to Equation 1.7 is the linear combination of the two principal solutions $C(s)=$ $\cos (\sqrt{K} s)$ and $S(s)=\frac{1}{\sqrt{K}} \sin (\sqrt{K} s)$ for $K>0$ or $C(s)=\cosh (\sqrt{-K} s)$ and $S(s)=\frac{1}{\sqrt{K}} \sinh (\sqrt{K} s)$ for $K<0$,

$$
y(s)=C(s) y_{0}+S(s) y_{0}^{\prime}, \quad y^{\prime}(s)=C^{\prime}(s) y_{0}+S^{\prime}(s) y_{0}^{\prime}
$$

where $y_{0}$ and $y_{0}^{\prime}$ are any initial phase space coordinates at $s_{0}$. It can be expressed in terms of the transfer matrix as

$$
\mathbf{y}(s)=M\left(s \mid s_{0}\right) \mathbf{y}\left(s_{0}\right), \quad M\left(s \mid s_{0}\right)=\left(\begin{array}{cc}
C(s) & S(s) \\
C^{\prime}(s) & S^{\prime}(s)
\end{array}\right), \quad \mathbf{y}(s)=\left(\begin{array}{c}
y(s) \\
y^{\prime}(s)
\end{array}\right)
$$


For instance, the transfer matrices for linear magnetic elements are,

$$
M\left(s \mid s_{0}\right)=\left\{\begin{array}{cc}
\left(\begin{array}{cc}
\cos \sqrt{K} l & \frac{1}{\sqrt{K}} \sin \sqrt{K} l \\
-\sqrt{K} \sin \sqrt{K} l & \cos \sqrt{K} l
\end{array}\right) & K>0, \text { focusing quad } \\
\left(\begin{array}{cc}
\cosh \sqrt{-K} l & \frac{1}{\sqrt{-K}} \sinh \sqrt{-K} l \\
-\sqrt{-K} \sinh \sqrt{-K} l & \cosh \sqrt{-K} l
\end{array}\right) & K<0, \text { defocusing quad } \\
\left(\begin{array}{cc}
1 & l \\
0 & 1
\end{array}\right) & K=0 \text {, drift space } \\
\left(\begin{array}{cc}
\cos \theta & \rho \sin \theta \\
-\frac{1}{\rho} \sin \theta & \cos \theta
\end{array}\right) & K=0, \text { pure sector dipole }
\end{array}\right.
$$

where $l=s-s_{0}$ is the length of the magnet, $\theta$ is the bending angle of the sector dipole, $\rho$ is the bending radius. A thin lens approximation with $f=1 /(|K| l)$ reduces the matrices to

$$
\mathbf{M}_{\mathbf{F}}=\left(\begin{array}{cc}
1 & 0 \\
-\frac{1}{f} & 1
\end{array}\right), \quad \mathbf{M}_{\mathbf{D}}=\left(\begin{array}{cc}
1 & 0 \\
\frac{1}{f} & 1
\end{array}\right)
$$

The most general form of $\mathbf{M}$ can be written as

$$
\mathbf{M}=\left(\begin{array}{cc}
\cos \Phi+\alpha \sin \Phi & \beta \sin \Phi \\
-\gamma \sin \Phi & \cos \Phi-\alpha \sin \Phi
\end{array}\right)=\mathbf{I} \cos \Phi+\mathbf{J} \sin \Phi
$$

where $\alpha, \beta$ and $\gamma$ are called the Courant - Snyder (C-S) parameters, $\Phi$ is the phase advance, $\mathbf{I}$ is the unit matrix. The matrix $\mathbf{J}$ satisfies $\operatorname{Trace}(\mathbf{J})=0$, and $\mathbf{J}^{2}=-\mathbf{I}$ or $\beta \gamma=1+\alpha^{2}$. The C-S parameters at $s_{2}$ are related to those at $s_{1}$ by

$$
\left(\begin{array}{c}
\beta \\
\alpha \\
\gamma
\end{array}\right)_{2}=\left(\begin{array}{ccc}
M_{11}^{2} & -2 M_{11} M_{12} & M_{12}^{2} \\
-M_{11} M_{21} & M_{11} M_{22}+M_{12} M_{21} & -M_{12} M_{22} \\
M_{21}^{2} & -2 M_{21} M_{22} & M_{22}^{2}
\end{array}\right)\left(\begin{array}{l}
\beta \\
\alpha \\
\gamma
\end{array}\right)_{1}
$$


where $M_{i j}$ are the matrix elements of $M\left(s_{2} \mid s_{1}\right)$. Using the Floquet transformation, the solution of Equation (1.7) can be expressed as a linear combination of two independent solutions $y(s)=a w(s) e^{i \psi(s)}$ and $y^{*}(s)=a w(s) e^{-i \psi(s)}$, where $w(s)$ and $\psi(s)$ satisfy the betatron envelope and phase equations, $w^{\prime \prime}+K w-1 / w^{3}=0, \psi^{\prime}=1 / w^{2}$. Checking the transfer matrix $\mathbf{M}$ for a complete period, using the Floquet transformation and Equation (1.12), gives the relations as follows.

$$
w^{2}=\beta, \quad \alpha=-w w^{\prime}=-\beta^{\prime} / 2
$$

This implies that the $\mathrm{C}-\mathrm{S}$ parameter $\beta(s)$ can be referred to as the betatron amplitude function, which is the square of the betatron motion amplitude. The betatron phase advance of one period is $\Phi=\int_{0}^{L} \frac{d s}{\beta(s)}$. With these relations, the transfer matrix from $s_{1}$ to $s_{2}$ becomes

$$
M\left(s_{2} \mid s_{1}\right)=\left(\begin{array}{cc}
\sqrt{\frac{\beta_{2}}{\beta_{1}}}\left(\cos \psi+\alpha_{1} \sin \psi\right) & \sqrt{\beta_{1} \beta_{2}} \sin \psi \\
-\frac{1+\alpha_{1} \alpha_{2}}{\sqrt{\beta_{1} \beta_{2}}} \sin (\psi)+\frac{\alpha_{1}-\alpha_{2}}{\sqrt{\beta_{1} \beta_{2}}} \cos \psi & \sqrt{\frac{\beta_{1}}{\beta_{2}}}\left(\cos \psi-\alpha_{2} \sin \psi\right)
\end{array}\right)
$$

The general solution of Equation (1.7) can be expressed now as

$$
y(s)=a \sqrt{\beta_{y}(s)} \cos \left[\psi_{y}(s)+\phi_{y}\right], \quad \psi_{y}(s)=\int_{0}^{s} \frac{d s}{\beta_{y}(s)}
$$

where $a$ and $\phi_{y}(s)$ are integration constants described by the initial condition. The betatron tune $\nu_{y}$ is defined as the number of betatron oscillations in one revolution, or namely $\nu_{y}=\frac{1}{2 \pi} \int_{s}^{s+C} \frac{d s}{\beta_{y}(s)}$, where $C$ is the circumference of the revolution.

In order to use $\psi$ as the angle-coordinate, a pseudo-Hamiltonian, $H=\frac{1}{2} y^{\prime 2}+$ $\frac{1}{2} K(s) y^{2}$ can be used to derive the Hill's equation. With the generating function $F_{1}(y, \psi)=\int_{0}^{y} y^{\prime} d s$, the new Hamiltonian can be organized as $\tilde{H}=H+\frac{\partial F_{1}}{\partial s}=\frac{J}{\beta}$ so that the solutions of the Hill's equation are

$$
y(s)=\sqrt{2 \beta J} \cos \psi, \quad y^{\prime}(s)=-\sqrt{\frac{2 J}{\beta}}[\sin \psi+\alpha \cos \psi]
$$




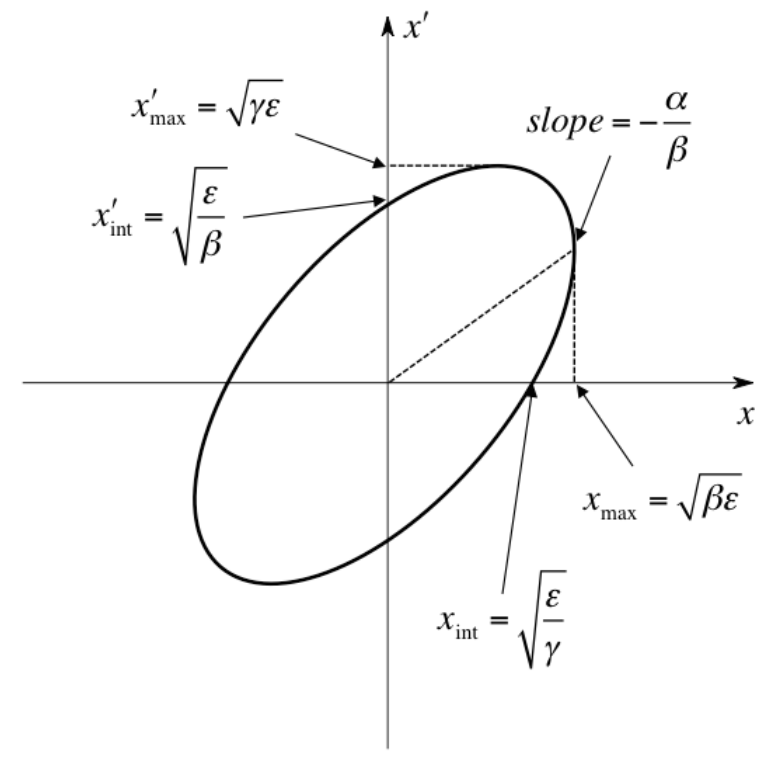

Figure 1.3: Invariant ellipse of the particle motion in the phase space.

where $J=\frac{1}{2 \beta}\left[y^{2}+\left(\beta y^{\prime}+\alpha y\right)^{2}\right]$ is the action variable, and $2 \pi J=\pi \epsilon$ describes the phase space area the betatron motion encloses. $\epsilon$ is conventionally referred to as the emittance of the particle. Since the new Hamiltonian does not depend on the angle variable $\psi, \epsilon$ is invariant of $s$. The particle trajectory in the phase space is an ellipse defined by

$$
\epsilon=\gamma y^{2}+2 \alpha y y^{\prime}+\beta y^{\prime 2}
$$

The maximum angular divergence and amplitude of the motion are described by $\sqrt{\gamma \epsilon}$ and $\sqrt{\beta \epsilon}$, respectively. The shape of the ellipse is drawn in Figure 1.3.

\subsubsection{Particle Beam}

Consider a multi-particle beam, where its particles are distributed in phase space. According to the above section, each of the particles has its own invariant C-S ellipse, 
when only linear elements such as dipoles and quadrupoles are used in the beamline. Consider a beam of particles in a 2-D phase space, for which the single particle emittance is given by Equation (1.18). The rms emittance of the beam is defined as

$$
\epsilon_{\mathrm{rms}}=\sqrt{\sigma_{11}^{2} \sigma_{22}^{2}-\sigma_{12}^{2}}
$$

where $\sigma$ is the sigma matrix of the beam, defined by

$$
\boldsymbol{\sigma}=\left(\begin{array}{ll}
\sigma_{11} & \sigma_{12} \\
\sigma_{21} & \sigma_{22}
\end{array}\right)=\left(\begin{array}{cc}
\sigma_{x}^{2} & \sigma_{x x^{\prime}} \\
\sigma_{x x^{\prime}} & \sigma_{x^{\prime}}^{2}
\end{array}\right) .
$$

Applying the coordinate transformation in Equation (1.17), and remembering that $\beta \gamma=1+\alpha^{2}$, the components of the sigma matrix can be obtained as

$$
\begin{aligned}
& \sigma_{11}=\left\langle x_{i}^{2}\right\rangle=\epsilon_{\mathrm{rms}} \beta \\
& \sigma_{12}=\left\langle x_{i} x_{i}^{\prime}\right\rangle=-\epsilon_{\mathrm{rms}} \alpha \\
& \sigma_{22}=\left\langle x_{i}^{\prime 2}\right\rangle=\epsilon_{\mathrm{rms}} \gamma
\end{aligned}
$$

where the subscript $i$ represents the properties for the $i$ th particle. With $\mathbf{x}=\left(x, x^{\prime}\right)$, the equation of motion of reach particle becomes

$$
\mathbf{x} \boldsymbol{\sigma}^{-1} \mathbf{x}=\frac{1}{\epsilon_{\mathrm{rms}}}\left(\gamma x^{2}+2 \alpha x x^{\prime}+\beta x^{2}\right) .
$$

For a bivariate Gaussian beam, the probability density function of its variables is

$$
f\left(x, x^{\prime}\right)=\frac{1}{2 \pi \sqrt{|\boldsymbol{\sigma}|}} e^{-\frac{1}{2|\boldsymbol{\sigma}|}\left(\sigma_{22} x^{2}-2 \sigma_{12} x x^{\prime}+\sigma_{11} x^{\prime 2}\right)} .
$$

With all the above equations considered, the function readily shows

$$
f(\epsilon)=\frac{1}{2 \epsilon_{\mathrm{rms}}} e^{-\epsilon / 2 \epsilon_{\mathrm{rms}}}
$$

The fraction of particles contained within $\epsilon=n \epsilon_{\mathrm{rms}}$ is then $1-e^{-n / 2}$. A conventional assumption is that the admittance of the machine, which is the maximum phase-space area that particles can survive, equals $6 \epsilon_{\mathrm{rms}}$. 


\subsubsection{Off-momentum Orbit}

A particle beam is usually composed of particles within a range of momenta centered at the design momentum $p_{0}$. For a particle with momentum $p$, the momentum deviation is $\Delta p=p-p_{0}$, and the fractional momentum deviation is then $\delta=\Delta p / p_{0}$. Expanding Equation (1.6) gives,

$$
x^{\prime \prime}+\left(\frac{1-\delta}{\rho^{2}(1+\delta)}-\frac{K(s)}{(1+\delta)}\right) x=\frac{\delta}{\rho(1+\delta)} .
$$

The solution of this inhomogeneous equation can be expressed as a linear superposition of the particular solution and the solution of the homogeneous equation, namely $x=x_{\beta}(s)+D(s) \delta$, where $x_{\beta}$ and $D$ are the solutions of

$$
\begin{aligned}
& x_{\beta}^{\prime \prime}+\left(K_{x}(s)+\Delta K_{x}\right) x_{\beta}=0 \\
& D^{\prime \prime}+\left(K_{x}(s)+\Delta K_{x}\right) D=\frac{1}{\rho}+O(\delta)
\end{aligned}
$$

where

$$
K_{x}=\frac{1}{\rho^{2}}-K(s)
$$

and

$$
\Delta K_{x}=\left[-\frac{2}{\rho^{2}}+K(s)\right] \delta+O\left(\delta^{2}\right) .
$$

If the chromatic perturbation term $\Delta K_{x}(s)$ can be ignored, $x_{\beta}$ is the betatron motion around the off-momentum closed orbit. Equations (1.28) can be solved using the matrix method

$$
\left(\begin{array}{c}
D\left(s_{2}\right) \\
D^{\prime}\left(s_{2}\right) \\
1
\end{array}\right)=\left(\begin{array}{cc}
M\left(s_{2} \mid s_{1}\right) & \bar{d} \\
0 & 1
\end{array}\right)\left(\begin{array}{c}
D\left(s_{1}\right) \\
D^{\prime}\left(s_{1}\right) \\
1
\end{array}\right)
$$


where $\bar{d}$ is

$$
\begin{aligned}
& \left(\begin{array}{c}
0 \\
0
\end{array}\right) \quad \text { for drift spaces and quadrupoles } \\
& \left(\begin{array}{c}
\frac{1}{\rho K_{x}}\left(1-\cos \sqrt{K_{x}} s\right) \\
\frac{1}{\rho \sqrt{K_{x}}} \sin \sqrt{K_{x}} s
\end{array}\right) \quad \text { for dipoles with } K_{x}>0 \\
& \left(\begin{array}{c}
\frac{1}{\rho\left|K_{x}\right|}\left(1-\cosh \sqrt{\left|K_{x}\right|} s\right) \\
\frac{1}{\rho \sqrt{\left|K_{x}\right|}} \sinh \sqrt{\left|K_{x}\right|} s
\end{array}\right) \quad \text { for dipoles with } K_{x}<0 .
\end{aligned}
$$

For instance, the transfer matrix for a pure sector dipole is

$$
\left(\begin{array}{ccc}
\cos \theta & \rho \sin \theta & \rho(1-\cos \theta) \\
-(1 / \rho) \sin \theta & \cos \theta & \sin \theta \\
0 & 0 & 1
\end{array}\right)
$$

where $\theta$ is the bend angle.

\subsubsection{Momentum compaction factor}

The path length difference between a circulating particle and a reference particle can be expressed by

$$
\Delta C=\oint \frac{x}{\rho} d s
$$

For an off-momentum particle this becomes

$$
\Delta C=\left[\oint \frac{D(s)}{\rho} d s\right] \delta .
$$

The momentum compaction factor is defined as

$$
\alpha_{c} \equiv \frac{1}{C} \frac{d \Delta C}{d \delta}=\frac{1}{C} \oint \frac{D(s) d s}{\rho} .
$$

Correspondingly, the phase-slip factor $\eta$ is defined as,

$$
\eta=-\frac{\Delta f}{f_{0}} \frac{1}{\delta}=-\left(-\frac{\Delta C}{C}+\frac{\Delta v}{v}\right) \frac{1}{\delta}=\left(\alpha_{c}-\frac{1}{\gamma^{2}}\right) \frac{\Delta p}{p_{0}} \frac{1}{\delta}=\left(\alpha_{c}-\frac{1}{\gamma^{2}}\right) .
$$


The transition- $\gamma$ is defined as $\gamma_{T} \equiv \sqrt{1 / \alpha_{c}}$, so that $\eta=1 / \gamma_{T}^{2}-1 / \gamma^{2}$. Equation (1.37) implies that the particles with higher momentum will revolve faster than a synchronous particle at below the transition energy $\left(\gamma<\gamma_{T}\right)$ and the opposite is true when it's above the transition energy $\left(\gamma>\gamma_{T}\right)$. When $\gamma=\gamma_{T}$, it is called the isochronous condition, at which point all the particles have the same revolution frequency.

\subsection{Synchrotron Motion}

RF cavities accelerate or decelerate particles by providing longitudinal electric field at a frequency $f_{r f}$. In order to provide in-phase acceleration or deceleration, the frequency $f_{r f}$ is synchronized with the revolution frequency $\left(f_{0}\right)$ of the reference particle with momentum $p_{0}$ so that $f_{r f}=h f_{0}$, where $h$ is called the harmonic number. The synchronous phase of the reference particle is normally denoted as $\phi_{s}$, and the phase of a particle with momentum $p$ is denoted by $\phi$. The linearized synchrotron equation of motion is denoted by [4],

$$
\frac{d^{2}}{d t^{2}}\left(\phi-\phi_{s}\right)=\frac{h \omega_{0}^{2} e V \eta \cos \phi_{s}}{2 \pi \beta^{2} E_{0}}\left(\phi-\phi_{s}\right)
$$

where $V$ is the maximum RF voltage, $\omega_{0}$ and $E_{0}$ are the revolution frequency and the energy of the synchronous particle. The simple differential equation in (1.38) yields a stable solution for $\left(\phi-\phi_{s}\right)$ when

$$
\left\{\begin{array}{l}
0 \leq \phi_{s} \leq \pi / 2, \quad \eta<0 \\
\pi / 2 \leq \phi_{s} \leq \pi, \quad \eta>0
\end{array}\right.
$$

Equation (1.39) is physically meaningful, since low energy particles will arrive later than the synchronous particle when $\eta<0$ and thus gain more energy. The syn- 
chrotron tune is defined as the synchrotron oscillation frequency divided by $\omega_{0}$ :

$$
Q_{s}=\frac{\omega_{s y n}}{\omega_{0}}=\sqrt{\frac{h e V_{0}\left|\eta \cos \phi_{s}\right|}{2 \pi \beta^{2} E_{0}}} .
$$




\section{CHAPTER 2}

\section{INTRODUCTION TO NEUTRINOS AND NUSTORM}

In this chapter, the current basic understanding of neutrinos is introduced, which will lead to the physics motivation for nuSTORM. This chapter will cover historical findings about neutrinos, and also some of the current neutrino experimental results that are closely related to nuSTORM. As it is the accelerator physics that is the major topic of the thesis, only a basic discussion of neutrino physics will be given. The study of neutrinos is a very wide and deep topic that has been highly active since 1930.

\subsection{History of Neutrinos}

In late 1930, W. Pauli proposed a new subatomic particle that shares the decay energy with the electron in beta-decay $\left(\beta-\right.$ decay: $(Z, A) \rightarrow(Z+1, A)+e^{-}+\bar{\nu}_{e}, \beta+$ decay: $\left.(Z, A) \rightarrow(Z-1, A)+e^{+}+\nu_{e}\right)$. To produce the observed energy spectrum, this new particle, which was later named the neutrino (by E. Fermi), could have a mass no larger than that of the electron. It has no electric charge and it has half-integer spin, which is the definition of a fermion. It obeys the Pauli exclusion principle that forbids two identical fermions to be in the same state at the same time.

Later, E. Fermi proposed a theory that a weak force, which is dramatically weaker 
than the electromagnetic force, turns a neutron into a proton and simultaneously creates an electron and an anti-neutrino. The theory explained all the observed properties of $\beta$ decay. Shortly thereafter, H. Bethe and R. Peierls used the weak force to predict that the neutrinos can be stopped through the inverse beta decay process, that a neutrino could be captured by a nucleus with the emission of an electron or a positron.

The description of this inverse beta-decay process can be described by

$$
\bar{\nu}+N(Z, A) \rightarrow e^{+}+X(Z-1, A) .
$$

If a hydrogen nucleus is used, then this simply turns into

$$
\bar{\nu}+p \rightarrow e^{+}+n
$$

Scientists on the Savannah River Experiments announced the data in 1956 that confirmed the discovery of neutrinos. The experimental results also agreed to within $5 \%$ with the theoretically predicted value for the inverse-beta-decay cross section. Another experiment in 1963 proved that a second (muon) neutrino was paired with the muon, similar to the pairing of the known (electron) neutrino with the electron, and that the muon neutrino is not identical to the electron neutrino. Later, S. Glashow at al. proposed the theory with a Z-boson, which predicted the existence of neutral currents (NC) events first observed in the Gargamelle experiment in $1973\left(\nu_{\mu}+N \rightarrow\right.$ $\nu_{\mu}+X, \bar{\nu}_{\mu}+N \rightarrow \bar{\nu}_{\mu}+X$, where $\mathrm{X}$ stands for the final hadronic state). Charged current (CC) events $\left(\nu_{\mu}+N \rightarrow \mu^{-}+X, \bar{\nu}_{\mu}+N \rightarrow \mu^{+}+X\right)$ were also detected along with the NC events. Afterwards, purely leptonic NC events $\left(\bar{\nu}_{\mu}+e \rightarrow \bar{\nu}_{\mu}+e\right)$ were also discovered.

In 1987, neutrinos were observed from one of the most important events in the history of astronomy, supernova (SN) 1987A. It was recorded as the brightest SN since 
Kepler's SN in 1604. The SN 1987A was the first time neutrinos were observed from an astrophysical source other than the Sun. Experiments also discovered that the electron neutrinos arriving at the Earth from the Sun were too few in number, which indicated that the electron neutrinos had changed into another flavor, either muon or tau neutrinos. It also suggested that the neutrinos have masses, which differ among the neutrino flavors. In the 1990s, the LSND (Liquid Scintillator Neutrino Detector) experiment observed data consistent with a neutrino oscillation, into a sterile state that is not part of Standard Model (SM) lepton families.

\section{$2.2 \quad$ Basic Neutrino Properties}

\subsubsection{Neutrinos in the Standard Model}

There are 12 basic building blocks of matter in the SM, 6 quarks ( $\mathrm{u}, \mathrm{c}, \mathrm{t}, \mathrm{d}, \mathrm{s}, \mathrm{b})$ and 6 leptons $\left(\nu_{e}, \nu_{\mu}, \nu_{\tau}, \mathrm{e}, \mu, \tau\right)$, all having half-integer spin and identified as fermions. Quarks have quantized electric charge and interact primarily through the strong nuclear force. Together with gluons they form the neutron, the proton, and all nuclei and hadrons that interact through the strong force. Charged leptons interact primarily through weak and electromagnetic forces. Therefore, leptons are not bound in the nucleus through the strong force. Neutrinos, with no electric charge, interact only through the weak force. There are 4 gauge particles, gluons, $\mathrm{Z}$ and $\mathrm{W}$ bosons, and the $\gamma$ that carry the forces of strong, weak, and electromagnetic interactions. They have integer spins, so are bosons. The recently discovered spin 0 boson that causes particle mass states and has spin of 0 , the Higgs boson $(\mathrm{H})$, is another building block of the SM. The chart of all the Standard Model particles is shown in Figure 2.1.

The Higgs mechanism implies that the mass states of neutrinos are mixtures of 


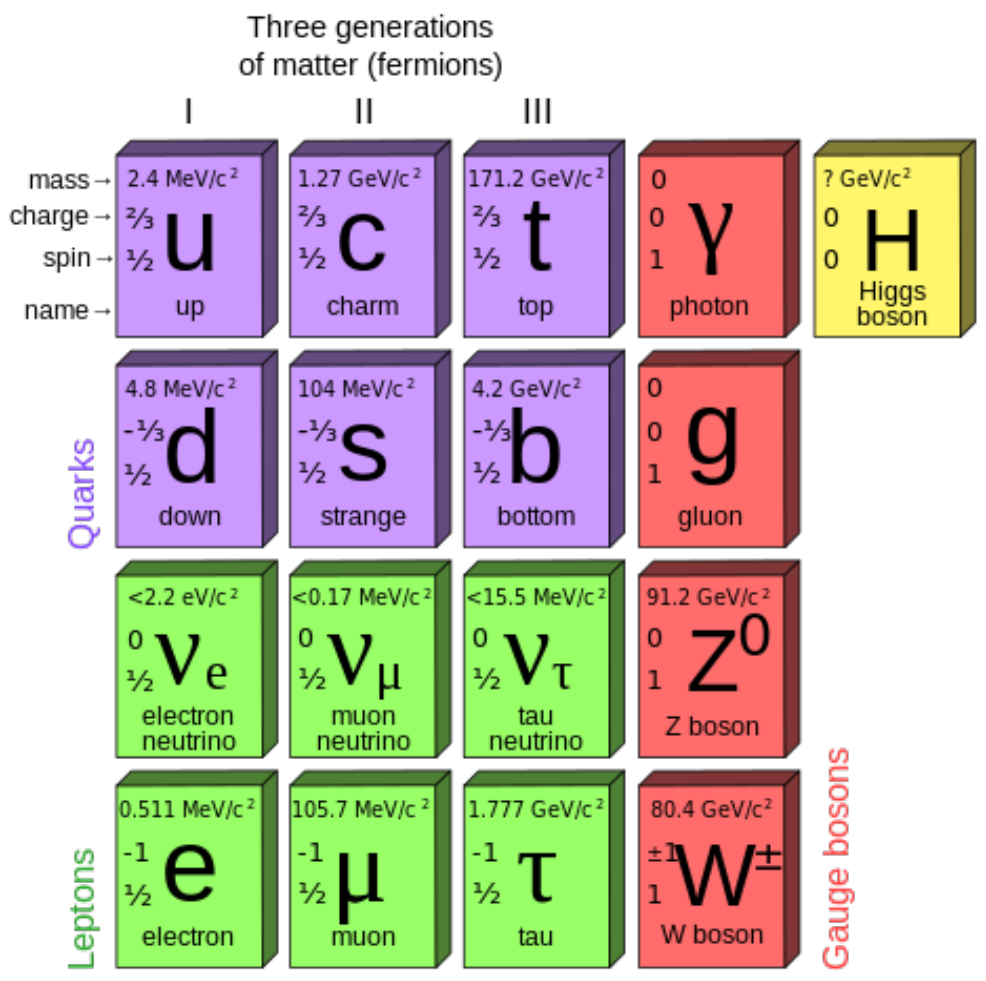

Figure 2.1: The standard model particles. The mass for the Higgs boson was measured by CMS and ATLAS, to be $125.03_{-0.27}^{+0.26}$ (stat) ${ }_{-0.15}^{+0.13}$ (sys) and $125.36 \pm 0.37$ (stat) \pm 0.18 (sys) $\mathrm{GeV} / \mathrm{c}^{2}[5,6]$ (Courtesy of schools-wikipedia.org)

the flavor states. However, it has not been determined if the neutrinos acquire masses through the Higgs mechanism or the Majorana mechanism, in which the neutrino is its own anti-particle. Neutrino oscillation measurements will provide information on neutrino mass and mixing. 


\begin{tabular}{cllll}
\hline Particle & Lepton & Electron- & Muon- & Tau- \\
& Number & Family & Family & Family \\
& $\boldsymbol{L}$ & Number & Number & Number \\
& & $\boldsymbol{L}_{\boldsymbol{e}}$ & $\boldsymbol{L}_{\boldsymbol{\mu}}$ & $\boldsymbol{L}_{\boldsymbol{\tau}}$ \\
& & & & \\
\hline$e^{-}$ & +1 & +1 & 0 & 0 \\
$\nu_{e}$ & +1 & +1 & 0 & 0 \\
$e^{+}$ & -1 & -1 & 0 & 0 \\
$\bar{\nu}_{e}$ & -1 & -1 & 0 & 0 \\
$\mu^{-}$ & +1 & 0 & +1 & 0 \\
$\nu_{\mu}$ & +1 & 0 & +1 & 0 \\
$\mu^{+}$ & -1 & 0 & -1 & 0 \\
$\bar{\nu}_{\mu}$ & -1 & 0 & -1 & 0 \\
$\tau^{-}$ & +1 & 0 & 0 & +1 \\
$\nu_{\tau}$ & +1 & 0 & 0 & +1 \\
$\tau^{+}$ & -1 & 0 & 0 & -1 \\
$\bar{\nu}_{\tau}$ & -1 & 0 & 0 & -1 \\
\hline
\end{tabular}

Table 2.1: The Lepton and Lepton-Family Numbers of leptons

\subsubsection{Conservation of Total Lepton Number and Lepton-Family number}

In the baseline Standard Model, there is no mixing among the leptons and the neutrinos from different lepton families can not transmute into each other. This suggests there are two numbers that are conserved during weak interactions, which are the total lepton number and the individual lepton-family number. The list of lepton numbers and lepton-family numbers are shown in Table 2.1. The conservation laws forbid the decays like $\mu^{+} \rightarrow e^{+}+\gamma$, and $\mu^{+} \rightarrow e^{+}+e^{-}+e^{+}$. 


\subsubsection{Neutrino Mixing}

If the neutrinos are massive, the neutrino flavor states $\nu_{e}, \nu_{\mu}$ and $\nu_{\tau}$ are linear combinations of the mass eigenstates $\nu_{1}, \nu_{2}$, and $\nu_{3}$. The weak and mass eigenvectors are given by

$$
\nu_{W} \equiv\left(\begin{array}{c}
\nu_{e} \\
\nu_{\mu} \\
\nu_{\tau}
\end{array}\right)=U \nu_{M} \equiv U\left(\begin{array}{c}
\nu_{1} \\
\nu_{2} \\
\nu_{3}
\end{array}\right)
$$

where $U$ is a unitary matrix. The Pontecorvo-Maki-Nakagawa-Sakata matrix (PMNS matrix) representation of $U$ for neutrinos has the following form:

$$
U=\left(\begin{array}{ccc}
c_{12} c_{13} & s_{12} c_{13} & s_{13} e^{-i \delta} \\
-s_{12} c_{23}-c_{12} s_{23} s_{13} e^{i \delta} & c_{12} c_{23}-s_{12} s_{23} s_{13} e^{i \delta} & s_{23} c_{13} \\
s_{12} s_{23}-c_{12} c_{23} s_{13} e^{i \delta} & -c_{12} s_{23}-s_{12} c_{23} s_{13} e^{i \delta} & c_{23} c_{13}
\end{array}\right)
$$

where $c_{i j}=\cos \theta_{i j}$ and $s_{i j}=\sin \theta_{i j}, i, j=1,2,3$.

\subsection{4 $C P$ Violation}

The $C P$ violation is directly related to the phase term $e^{i \delta}$ in the PMNS matrix. To be $C P$ invariant, the CKM matrix should be identical to its complex conjugate, which means $e^{i \delta}$ should be real. In the 3 -family mixing matrix, this is only true when $\delta=0, \pi$ or $2 \pi$. If $\delta \neq 0, \pi$ or $2 \pi, C P$ is violated. The "Neutrino Factory" (NF) [7] provides the most precise method to measure the phase of $C P$ violation in the leptonic sector. Instead of using neutrinos from decays of secondary particles, the NF uses the neutrinos from decays of muons that are injected into a storage ring. The muon decay, $\mu^{+} \rightarrow e^{+} \nu_{e} \bar{\nu}_{\mu}$, is well understood with the neutrino spectrum from 
$\mu^{+}$decay in the muon rest frame given by [8]

$$
\begin{aligned}
& \frac{d^{2} N_{\nu_{\mu}}}{d y d A}=\frac{4 n_{\mu}}{\pi L^{2} m_{\mu}^{6}} E_{\mu}^{4} y^{2}(1-\beta \cos \phi)\left[3 m_{\mu}^{2}-4 E_{\mu}^{2} y(1-\beta \cos \phi)\right] \\
& \frac{d^{2} N_{\nu_{e}}}{d y d A}=\frac{24 n_{\mu}}{\pi L^{2} m_{\mu}^{6}} E_{\mu}^{4} y^{2}(1-\beta \cos \phi)\left[m_{\mu}^{2}-2 E_{\mu}^{2} y(1-\beta \cos \phi)\right]
\end{aligned}
$$

where $y=E_{\nu} / E_{\mu}, n_{\mu}$ is the number of muons, $\beta$ is the relativistic factor, $A$ is an area, and $L$ is the longitudinal distance from the decay point to $A$.

\subsubsection{Neutrino Oscillations}

The theory of neutrino oscillation is based on the assumption that the lepton flavor states and lepton mass states are not the same. The neutrino starts in a pure flavor state, which can oscillate to a flavor state from a different lepton family. It is the mass states that the original flavor state are composed of that evolves in time.

If neutrinos are massive and mix, a neutrino field with flavor $\alpha(\alpha=e, \mu, \tau)$ is a linear combination of neutrino fields with definite masses, $\nu_{\alpha}(\alpha=1,2,3)$, given by [9]

$$
\nu_{\alpha}=\sum_{a=1,2,3} U_{\alpha a} \nu_{a}
$$

where $U_{\alpha a}$ are the matrix elements of a unitary matrix $U$. The time evolution of a mass eigenstate is given by

$$
\left|\psi_{a}^{M}(t)>=e^{-i H t}\right| \psi_{a}^{M}>=\int \frac{d \vec{p}}{(2 \pi)^{3 / 2}} \psi_{a}^{M}(\vec{p}) e^{-i E_{a} t} \mid \vec{p}>.
$$

where $H$ is the Hamiltonian. The coordinate representation of the above equation is

$$
\begin{aligned}
\psi_{a}^{M}(\vec{x}, t) & =<\vec{x} \mid \psi_{a}^{M}(t)> \\
& =\int \frac{d \vec{p}}{(2 \pi)^{3 / 2}} \psi_{a}^{M}(\vec{p}) e^{-i E_{a} t}<\vec{x} \mid \vec{p}> \\
& =\int \frac{d \vec{p}}{(2 \pi)^{3 / 2}} \psi_{a}^{M}(\vec{p}) e^{i \vec{p} \cdot \vec{x}-i E_{a} t} .
\end{aligned}
$$


A propagating mass eigenstate in the coordinate space is then

$$
\left|\Psi_{a}^{M}(\vec{x}, t)>=<\vec{x}\right| \Psi_{a}^{M}>=<\vec{x}\left|\psi_{a}^{M}(t)>\otimes\right| \nu_{a}>=\psi_{a}^{M}(\vec{x}, t) \mid \nu_{a}>
$$

The flavor state is the linear combination of the mass states, which can be expressed as

$$
\left|\nu_{\alpha}>=\sum_{a} U_{\alpha a}^{*}\right| \nu_{a}>
$$

Therefore, the propagating flavor state is finally given by

$$
\begin{aligned}
\mid \Psi_{a}^{W}(\vec{x}, t)> & =\sum_{a} U_{\alpha a}^{*} \mid \Psi_{a}^{M}(\vec{x}, t)> \\
& =\sum_{a} U_{\alpha a}^{*} \psi_{a}^{M}(\vec{x}, t) \mid \nu_{a}>
\end{aligned}
$$

A flavor state neutrino $\nu_{\alpha}$ that was created at the space-time origin is

$$
\left|\Psi_{a}^{W}(0,0)>=\sum_{a} U_{\alpha a}^{*} \psi_{a}^{M}(0,0)\right| \nu_{a}>
$$

It is now straightforward to obtain the probability of the transition from $\nu_{\alpha}$ to $\nu_{\beta}$ after time $t$ as follows

$$
\begin{aligned}
P(\alpha \rightarrow \beta ; t) & =\left|<\Psi_{a}^{W}(\vec{x}, t)\right| \Psi_{a}^{W}(0,0)>\left.\right|^{2} \\
& =\left|\sum_{a} U_{\beta a} U_{\alpha a}^{*} \psi_{a}^{M}(0,0) \psi_{a}^{M *}(\vec{x}, t)\right|^{2}
\end{aligned}
$$

where $\left\langle\nu_{a} \mid \nu_{b}\right\rangle=\delta_{a b}$ has been applied.

The plane wave formalism of the neutrino mass state can be given by the energy and momentum of the neutrino,

$$
\psi_{a}^{M}(\vec{x}, t)=e^{i \vec{p}_{a} \cdot \vec{x}-i E_{a} t}
$$


where $E_{a} \approx p_{a}+\frac{m_{a}^{2}}{2 p_{a}}$. Taking the direction of the neutrino propagation to be along the $x$-axis, the oscillation probability can be shown to be:

$$
\begin{aligned}
P(\alpha \rightarrow \beta ; t) & =\left|\sum_{a} U_{\beta a} e^{i p_{a}(x-t)-i \frac{m_{a}^{2}}{2 p_{a}} t} U_{\alpha a}^{*}\right|^{2} \\
& =\sum_{a}\left|U_{\alpha a}^{*}\right|^{2}\left|U_{\beta a}\right|^{2} \\
& +2 \operatorname{Re}\left[\sum_{a \neq b} U_{\alpha a}^{*} U_{\beta a} U_{\alpha b} U_{\beta b}^{*} e^{i\left(p_{a}-p_{b}\right)(x-t)-i \frac{\Delta m_{a b}^{2}}{2 p} t}\right] \\
& =\sum_{a}\left|U_{\alpha a}^{*}\right|^{2}\left|U_{\beta a}\right|^{2} \\
& +2 \operatorname{Re}\left[\sum_{a \neq b} U_{\alpha a}^{*} U_{\beta a} U_{\alpha b} U_{\beta b}^{*} e^{-i \frac{\Delta m_{a b}^{2}}{2} \frac{L}{E}}\right]
\end{aligned}
$$

where $\Delta m_{a b}^{2}=m_{a}^{2}-m_{b}^{2}$. The second term is spatially dependent. $C P$ violation is suggested when $P\left(\nu_{\alpha} \rightarrow \nu_{\beta}\right) \neq P\left(\bar{\nu}_{\alpha} \rightarrow \bar{\nu}_{\beta}\right)$, while $T$ violation is suggested when $P\left(\nu_{\alpha} \rightarrow \nu_{\beta}\right) \neq P\left(\nu_{\beta} \rightarrow \nu_{\alpha}\right)(\alpha \neq \beta)$. If $C P T$ is conserved, then violation of $C P$ is equivalent to violation of $T$.

In the context of 2-dimensions, where only $\nu_{e}$ and $\nu_{\mu}$ are considered, the unitary matrix $U$ is

$$
U^{*}=U=\left(\begin{array}{cc}
\cos \theta & \sin \theta \\
-\sin \theta & \cos \theta
\end{array}\right)
$$

The expressions for $\nu_{e}$ oscillation are then

$$
\begin{aligned}
P\left(\nu_{e} \rightarrow \nu_{e}\right) & =\left[1-\frac{1}{2} \sin ^{2}(2 \theta)\right]+\frac{1}{2} \sin ^{2}(2 \theta) \cos \left(\frac{\Delta m_{21}^{2} L}{2 E}\right) \\
& =1-\sin ^{2}(2 \theta) \sin ^{2}\left(\frac{\Delta m_{21}^{2} L}{4 E}\right) \\
P\left(\nu_{e} \rightarrow \nu_{\mu}\right) & =\frac{1}{2} \sin ^{2}(2 \theta)-\frac{1}{2} \sin ^{2}(2 \theta) \cos \left(\frac{\Delta m_{21}^{2} L}{2 E}\right) \\
& =\sin ^{2}(2 \theta) \sin ^{2}\left(\frac{\Delta m_{21}^{2} L}{4 E}\right)
\end{aligned}
$$




\begin{tabular}{clll}
\hline Parameter & Unit & $\begin{array}{l}3 \sigma \text { range } \\
\text { normal } \\
\text { mass } \\
\text { hierarchy }\end{array}$ & $\begin{array}{l}3 \sigma \text { inverse } \\
\text { normal } \\
\text { mass } \\
\text { hierarchy }\end{array}$ \\
& & & \\
\hline$\Delta m_{21}^{2}$ & $10^{-5} \mathrm{eV}^{2}$ & $7.12-8.20$ & $7.12-8.20$ \\
$\left|\Delta m_{31}^{2}\right|$ & $10^{-3} \mathrm{eV}^{2}$ & $2.31-2.74$ & $2.21-2.64$ \\
$\sin ^{2} \theta_{12}$ & 1 & $0.27-0.37$ & $0.27-0.37$ \\
$\sin ^{2} \theta_{23}$ & 1 & $0.36-0.68$ & $0.37-0.67$ \\
$\sin ^{2} \theta_{13}$ & 1 & $0.017-0.033$ & $0.017-0.033$ \\
$\delta$ & 1 & $0-2 \pi$ & $0-2 \pi$ \\
\hline
\end{tabular}

Table 2.2: Oscillation parameters for normal and inverted mass hierarchies.

The second sin term in Equations (2.17-2.18) can be rewritten in the following form when units of $L, \Delta m^{2}$ and $E$ are taken as $\mathrm{m}, \mathrm{eV}^{2}$ and $\mathrm{MeV}$, respectively:

$$
\sin ^{2}\left(\frac{\Delta m_{21}^{2} L}{4 E}\right)=\sin ^{2}\left(1.27 \frac{\Delta m_{21}^{2} L}{E}\right)
$$

Notice that this oscillation probability (2.18) also oscillates with $L$ and has a maximum value of $\sin ^{2} 2 \theta$. The oscillation happens when $\Delta m_{21}^{2}$ has a non-zero value. The violation of the lepton family number and the non-zero neutrino mass present physics beyond the Standard Model. Table 2.2 gives the current data on oscillation parameters for normal and inverted mass hierarchies. [10].

With non-zero mass, the neutrino might be a Dirac particle and would have 4 independent states $-\nu_{R}, \nu_{L}, \bar{\nu}_{R}$ and $\bar{\nu}_{L}$, where $R$ and $L$ stand for right-handedness and left-handedness, respectively. Two of these four states need to be added to the Standard Model if that be the case. The right-handed neutrino $\nu_{R}$ and the left-handed anti-neutrino $\bar{\nu}_{L}$ would be sterile neutrinos since they would not interact through any force except for gravity. $\nu_{R}$ and $\bar{\nu}_{L}$ would exist if the neutrinos are Dirac neutrinos. 
They differ in mass from the ordinary neutrino states, which could oscillate into the sterile states. If the neutrinos are Majorana particles, the left-handed $\nu_{L}$ and righthanded $\bar{\nu}_{R}$ are the only two states. They would transform to each other and be their own antiparticles.

\subsubsection{Sterile Neutrinos}

The solar and atmospheric neutrino oscillation experiments give two mass scales $\Delta m_{12}^{2}=8 \times 10^{-5} \mathrm{eV}^{2}$ and $\left|\Delta m_{23}^{2}\right|=2.3 \times 10^{-3} \mathrm{eV}^{2}$, respectively. The LSND result, however, suggests that there is another mass splitting, $\left|\Delta m_{L S N D}^{2}\right|=1.2 \mathrm{eV}^{2}$. LEP results, on the other hand, conclude that there are only 3 neutrinos, which implies (if all experimental results are correct) that there is at least one sterile neutrino that doesn't interact through the weak force. Other experiments, such as KARMEN, NOMAD, and miniBooNE, combined with the atmospheric and solar data, do not fit well with a one sterile neutrino $((3+1)$ model $)$. The $(3+2)$ and $(3+3)$ models do somewhat better.

In June, 2014, MINOS presented new results on their sterile neutrino search. The results in Figure 2.2 narrowed the possible region where the sterile neutrinos might exist. In order to do a more precise measurement, one could take advantage of the oscillation channels available at nuSTORM.

\subsubsection{Neutrino-Nucleon Scattering}

Neutrino-nucleus scattering consists of both charged-current interactions (CC), and neutral-current interactions (NC). In the charged-current interactions, the neutrino converts into its corresponding lepton (in the same family). The detection is then

performed by measuring the charged lepton. The quasi-elastic CC interactions are 


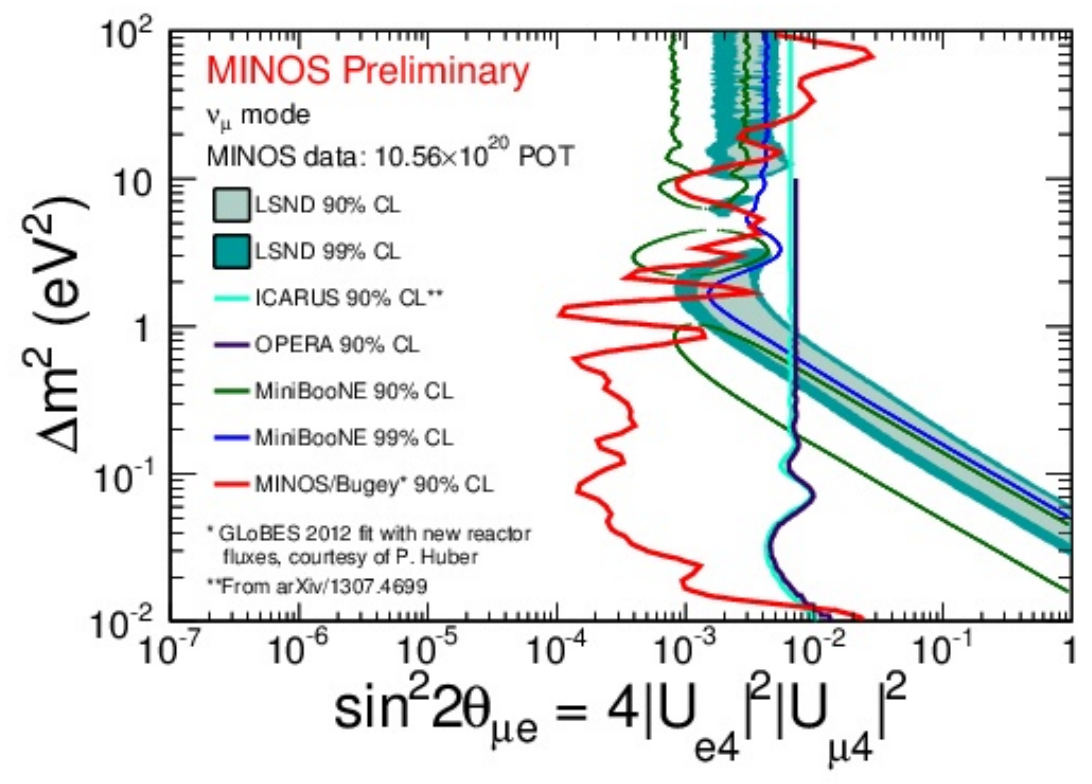

Figure 2.2: The sterile neutrino search from different experiments. The results from MINOS that were presented in June, 2014 exclude everything to the right of the red line. [11] 
listed below

$$
\begin{gathered}
\nu_{e}+n \rightarrow p+e^{-} \\
\nu_{\mu}+n \rightarrow p+\mu^{-} \\
\bar{\nu}_{e}+p \rightarrow n+e^{+} \\
\bar{\nu}_{\mu}+p \rightarrow n+\mu^{+} .
\end{gathered}
$$

In $\mathrm{NC}$ interactions, neutrinos are the outgoing leptons, when energy transfer takes place. CC interactions involve the exchange of either a $W^{+}$or a $W^{-}$boson, while $\mathrm{NC}$ interactions involve the exchange of a $Z^{0}$ boson. The $\mathrm{CC}$ interactions have the advantage in neutrino detection since they identify the neutrino flavor state through generating a lepton in the same family. The electrons or muons in the final state can then be identified in an appropriate particle detector.

\subsubsection{Total cross section for neutrino-nucleon scattering}

The total cross sections of muon neutrinos and antineutrinos have been measured by numerous experiments. The calculated and the measured values are plotted in Figure 2.3. However, there is still little data on $\nu_{e}$ cross sections in the few $\mathrm{GeV}$ range. Muon storage rings such as nuSTORM can provide the opportunity to explore this electron-neutrino energy regime.

\subsection{Introduction to nuSTORM}

Although a number of results have reported hints for neutrino oscillations involving sterile neutrinos, the experimented situation regarding their existence is far from clear. A systematically different and definitive experiment to confirm or refute their existence is warranted. nuSTORM is unique in that it can provide the definitive experimental search for sterile neutrinos and, furthermore, presents the possibility to 

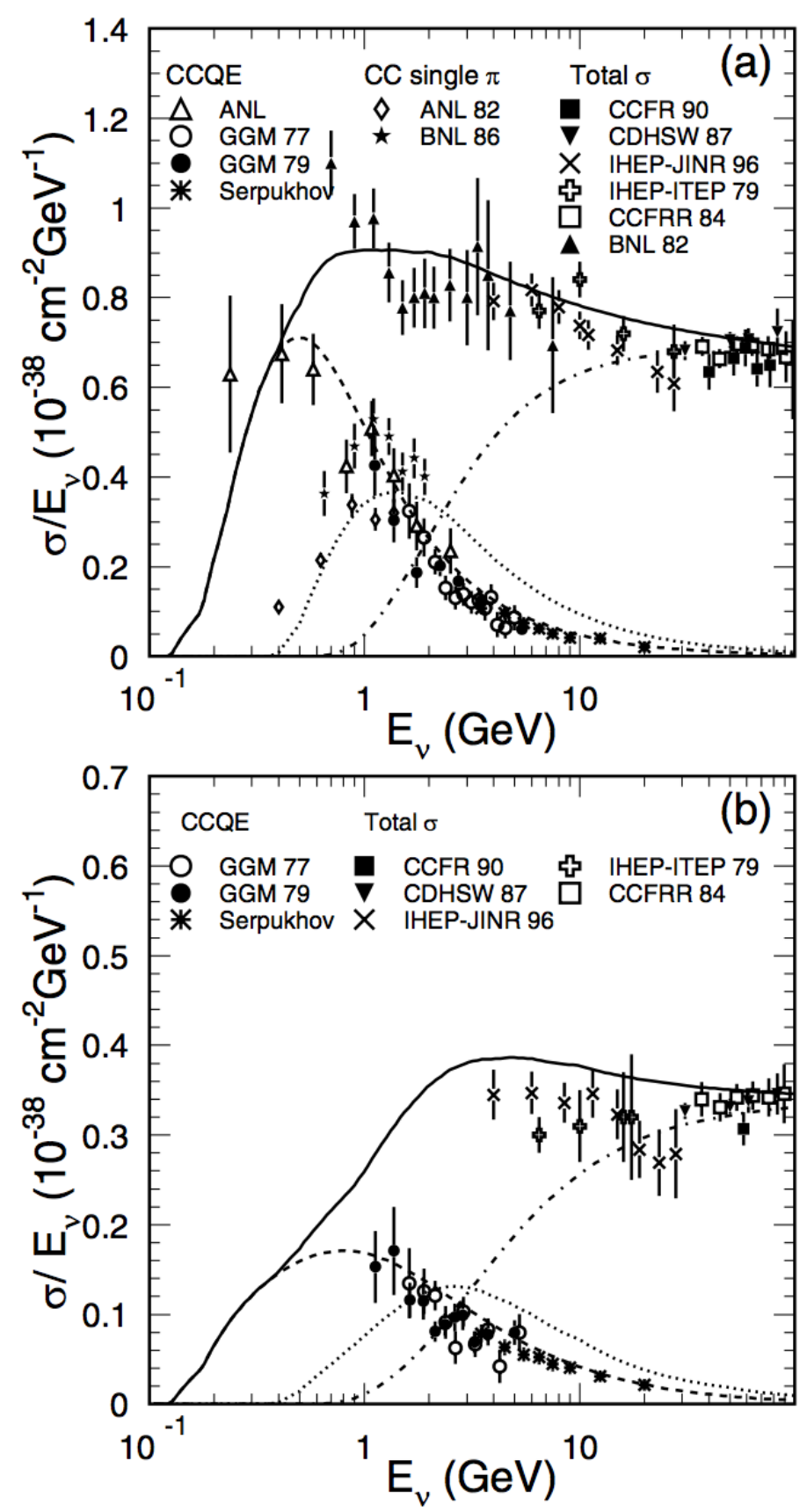

Figure 2.3: The total CC cross section for $\nu_{\mu}$ (upper) and $\bar{\nu}_{\mu}$ (lower) as a function of neutrino energy. Solid lines show the calculated total cross sections. The dashed, dot, and dash-dotted lines are the calculated quasi-elastic single-meson and deep-inelastic scatterings, respectively. [12] 
measure $\stackrel{c}{\nu}_{e} N$ and $\stackrel{c}{\nu}_{\mu} N$ interactions with a precision of $\simeq 1 \%$ over a neutrino energy range of $0.5<E_{\nu}<4 \mathrm{GeV}$.

nuSTORM is a unique short-baseline neutrino project, with three separate goals. It will allow searches for sterile neutrinos of extraordinary sensitivity, provide definitive measurement of $\stackrel{\complement}{\nu}_{e}$-nucleus and $\stackrel{C}{\nu}_{\mu}{ }_{\mu}$-nucleus cross sections with percent-level precision, and offer the next step in developing future muon accelerators. It provides a testbed for secondary beam detection, and possibly low energy muon cooling experiments, etc. Using a combination of a muon storage ring, and a near and a far magnetized iron neutrino detector (ND and FD) will enable searches for neutrino oscillations in both the appearance and disappearance channels. The statistics will be large enough at nuSTORM to completely exclude the presently allowed parameter space for $\stackrel{c}{\nu}_{e}$ oscillation in the LSND and MiniBooNE appearance channels. nuSTORM with its combined advantages of having instrumentation in the storage ring, knowing the neutrino flavor and energy spectrum precisely, and measuring the beam at a near detector allows the neutrino flux to be determined with $1 \%$ or better precision.

Last but not the least, the extraction configuration of nuSTORM will offer the opportunity to use the extracted muon beam for muon ionization cooling experiments. The demonstration of muon cooling is essential in the development of other muon accelerator facilities, such a neutrino factory, and ultimately a muon collider.

\subsubsection{Overview and Motivation}

nuSTORM by itself is the simplest representation of the Neutrino Factory (NF) concept [13]. In nuSTORM, pions are produced off a conventional solid target, which could be Carbon or Inconel, then are collected by a magnetic horn. A "pion beamline" 
that is composed of a series of linear magnets, namely dipoles and quadrupoles, then transports and injects the pion beam into the production straight section of the muon storage ring. The pions decay to muons and muon neutrinos while being transported in the pion beamline. Some of the muons generated in this process will be accepted within the muon storage ring acceptance. The accepted muons circulate in the storage ring and decay to electrons or positrons and corresponding neutrinos, depending on the muon flavor. Neutrinos produced in the straight section in which the direction of muons points to the detectors would be received by the detectors. Therefore, this straight section is referred to as the "production straight". The schematic drawing and the engineering layout of the nuSTORM facility is shown in Figure 2.4.

A near detector for oscillation measurements can be placed at 50 meters from the end of the production straight. It is identical to the far detector, but with only one tenth the fiducial mass. The far detector, at approximately $2 \mathrm{~km}$, will study the neutrino oscillations and search in both appearance and disappearance channels. If $\mu^{+}$are stored in the ring, the $\nu_{e} \rightarrow \nu_{\mu}$ oscillation, which is referred to as the golden channel, will result in the observation of $\mu^{-}$events in the detectors. To distinguish a $\mu^{-}$event from an ordinary $\mu^{+} \mathrm{CC}$ event from $\bar{\nu}_{\mu}$ in the beam, the detector must be magnetized. Therefore, the magnetized iron detector will be used.

The experiments that provide hints for neutrino oscillations involving light sterile neutrinos include LSND, MiniBooNE, and the reactor experiment anomaly. In LSND, the study of $\bar{\nu}_{e}$ to $\bar{\nu}_{\mu}$ oscillation resulted in the observation of $\bar{\nu}_{e}$ events with an experimental significance of more than $3 \sigma$. The corresponding mass splitting is $\Delta m^{2} \gtrsim 0.1 \mathrm{eV}^{2}$ and the corresponding $\bar{\nu}_{\mu}-\bar{\nu}_{e}$ mixing angle is $\sin ^{2} 2 \theta_{\mu e} \gtrsim 2 \times 10^{-3}$. In MiniBooNE, both the $\nu_{\mu}$ to $\nu_{e}$ and the $\bar{\nu}_{\mu}$ to $\bar{\nu}_{e}$ channels were searched for. The experiment also reported events with a significance of above $3 \sigma$ level, which are con- 

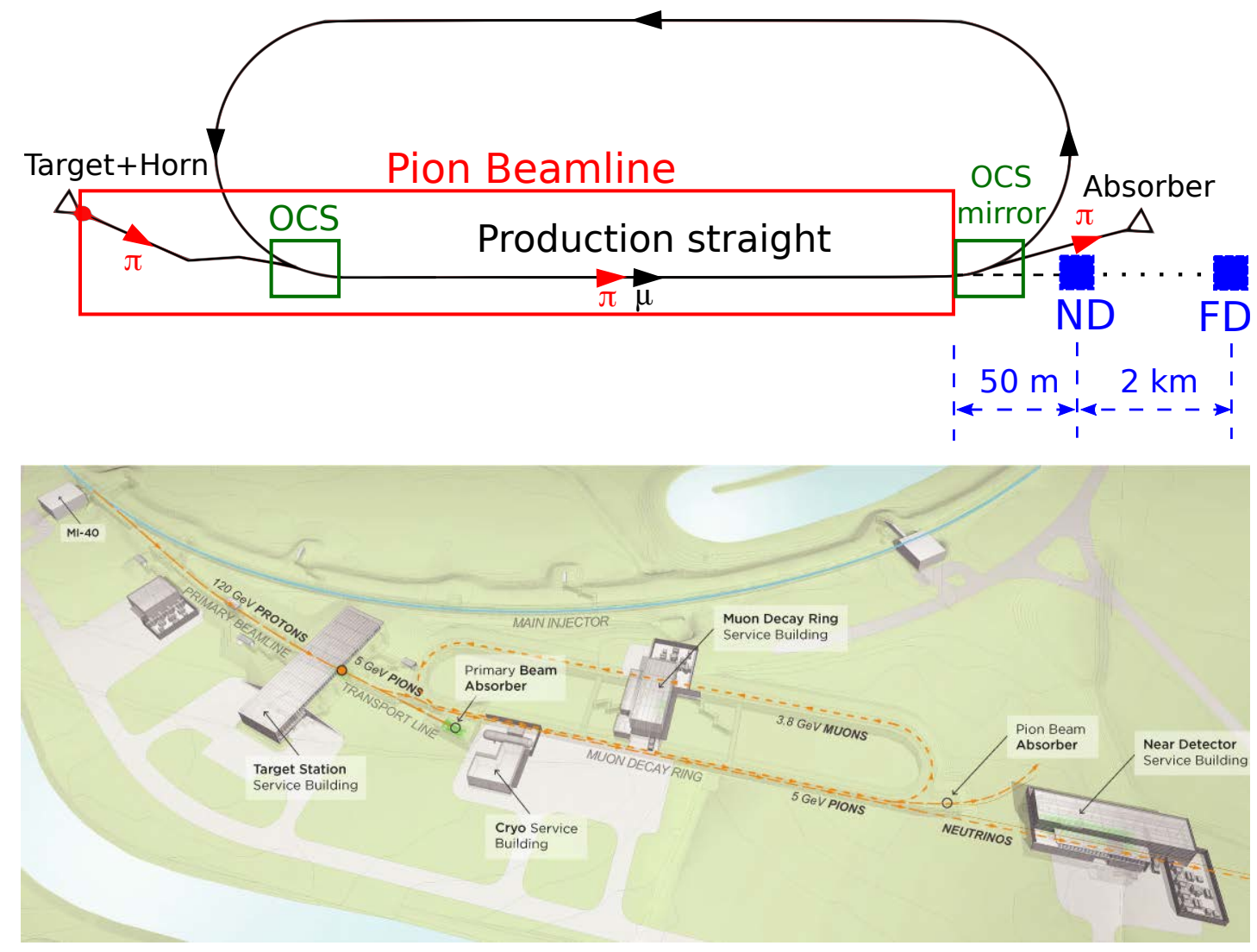

Figure 2.4: The schematic drawing and the engineering layout of the nuSTORM facility (upper and lower, respectively). 
sistent with the LSND result. If the reactor antineutrino anomaly is interpreted as $\bar{\nu}_{e} \rightarrow \bar{\nu}_{s}$ disappearance via oscillation, the corresponding mass split and mixing angle are $\Delta m^{2} \gtrsim 0.3 \mathrm{eV}^{2}$ and $\sin ^{2} 2 \theta_{e e} \sim 0.1$. The solar neutrino experiments GALLEX and SAGE both gave hints for $\bar{\nu}_{e} \rightarrow \bar{\nu}_{s}$ disappearance with $\Delta m^{2} \gtrsim 1 \mathrm{eV}^{2}$ and $\sin ^{2} 2 \theta_{e e} \sim 0.1-0.8$, at a significance level of around $3 \sigma$ from their calibration source $[14,15]$.

In a global fit of these data, including the results from MINOS, the solar neutrino experiments, etc., it is difficult to simultaneously accommodate all of these hints. Therefore, nuSTORM with its high sensitivity and precisely known systematic uncertainties has a great potential to either confirm a new type of neutrino oscillation or exclude this type of oscillation. nuSTORM has the capability to conduct both the muon appearance and disappearance measurements to thoroughly explore light sterile neutrinos.

Neutrino-nucleus interaction cross sections measurements are being performed by T2K, MiniBooNE, NOMAD, MINERvA, and MicroBooNE, etc. A main constraint on knowing these cross sections accurately is the knowledge of the neutrino flux. The accuracy MiniBooNE and NOMAD can obtain for the charged-current quasi-elastic cross section measurement is $\sim 8 \%$ for both experiments [16]. nuSTORM with its flux resolution of $\leq 1 \%$ can measure the $\mathrm{CC}$ quasi-elastic cross section to a precision that is nearly an order of magnitude smaller than either MiniBooNE or NOMAD. Other similar experiments attempting the same measurement also use a conventional muon beam from pion decay, where it is difficult to reach an accuracy of less than $5-7 \%$, compared with $\lesssim 1 \%$ in nuSTORM. 


\subsubsection{Introduction to the target and horn}

nuSTORM is designed to take one full booster proton batch from the Fermilab Main Injector (MI) every 1.33 seconds. The proton energy from the MI can range from 60 $\mathrm{GeV}$ to $120 \mathrm{GeV}$, while the pion production rate per proton in the pion momentum region of several $\mathrm{GeV} / \mathrm{c}$ increases proportionally with the proton energy. For the study presented in this thesis, the simulations were done based on $120 \mathrm{GeV}$ Protons On Target (POT). The proton beam pulse has $8 \times 10^{12}$ protons with a length of $1.6 \mu \mathrm{s}$, and a cycle period of 1.33 seconds. The beam has an RMS width of $1.1 \mathrm{~mm}$.

In selecting the material of the nuSTORM target, Graphite was chosen as the baseline due to its long use in the MINOS and MINER $\nu \mathrm{A}$ experiments. It also has favorable properties that minimize the dynamic stress waves and thermal stress gradients. It is able to survive at high temperatures in an environment that lacks oxidizing components. A Carbon target has been successfully operated for $6 \times 10^{20}$ POT at proton beam powers near $400 \mathrm{~kW}$ and $4.4 \times 10^{13}$ Protons Per Pulse (PPP) (NuMI target NT-02). Thus for the approximately $10^{21}$ POT exposure for nuSTORM, no target replacement is expected.

Efforts were made to investigate medium to high-Z target materials. More specifically, Inconel $718^{1}$ and Gold targets were studied to enhance the pion yield. It was realized that Gold and Inconel targets with the same interaction lengths can produce approximately $50 \%$ and $40 \%$ more pions in the momentum range of nuSTORM than the baseline Graphite target. However, since the energy deposition on the target and horn is proportional to $\mathrm{Z}$, the thermal load with Gold $(\mathrm{Z}=79)$ is about three times that of Inconel. The Inconel target analysis was performed via simulations, which showed tolerable energy deposition and thermal load/stress, when forced Helium cooling was

\footnotetext{
${ }^{1}$ a family of austenitic nickel-chromium-based superalloys
} 
used. Although further analysis is still needed to determine the optimized target geometry, the vibration modes of the target, etc., Inconel has produced promising results and was chosen to be the baseline target material in this thesis.

The magnetic horn is a focusing device commonly used to collect secondary particles from bombarding a target with a primary beam [17]. The "conventional horns" designed for NuMI and the Long Baseline Neutrino Facility (LBNF) were optimized for obtaining neutrinos from pion decay in a drift space after the horn collection $[18,19]$. For the case of nuSTORM, the primary goal is to collect the pions into a $2000 \mu \mathrm{m}$ phase space acceptance, so that they are captured by the pion beamline downstream the horn. The horn is designed to have a very thin inner conductor such that the off-axis pions produced from the target can penetrate through the conductor from the inner bore of the horn to the field region and get focused by the azimuthal field vector. The on-axis pions simply travel through the inner core that is field-free. A schematic drawing of the NuMI horn 1 and the LBNE horn 1 are shown in Figure 2.5.

The nuSTORM baseline horn design is based on the successful NuMI horn. It uses a configuration similar to the NuMI horn, with a modified horn length and target position in the horn. The schematic drawing of the horn and the target is shown in Figure 2.5, in which the scale in the drawing describes the real configuration in the simulation. This minimizes the cost of manpower by avoiding substantial redesigns. However, it is possible to keep the overall shape of the horn and modify configuration details such as the aperture sizes at the neck, at the entrance and exit of the horn, the length of the neck, and so forth. Details of the optimization study of the nuSTORM horn will be given in Chapter 4. With the previous target and horn settings, the phase distribution of the pions after collection by a NuMI-like horn is plotted in Figure 2.6. 

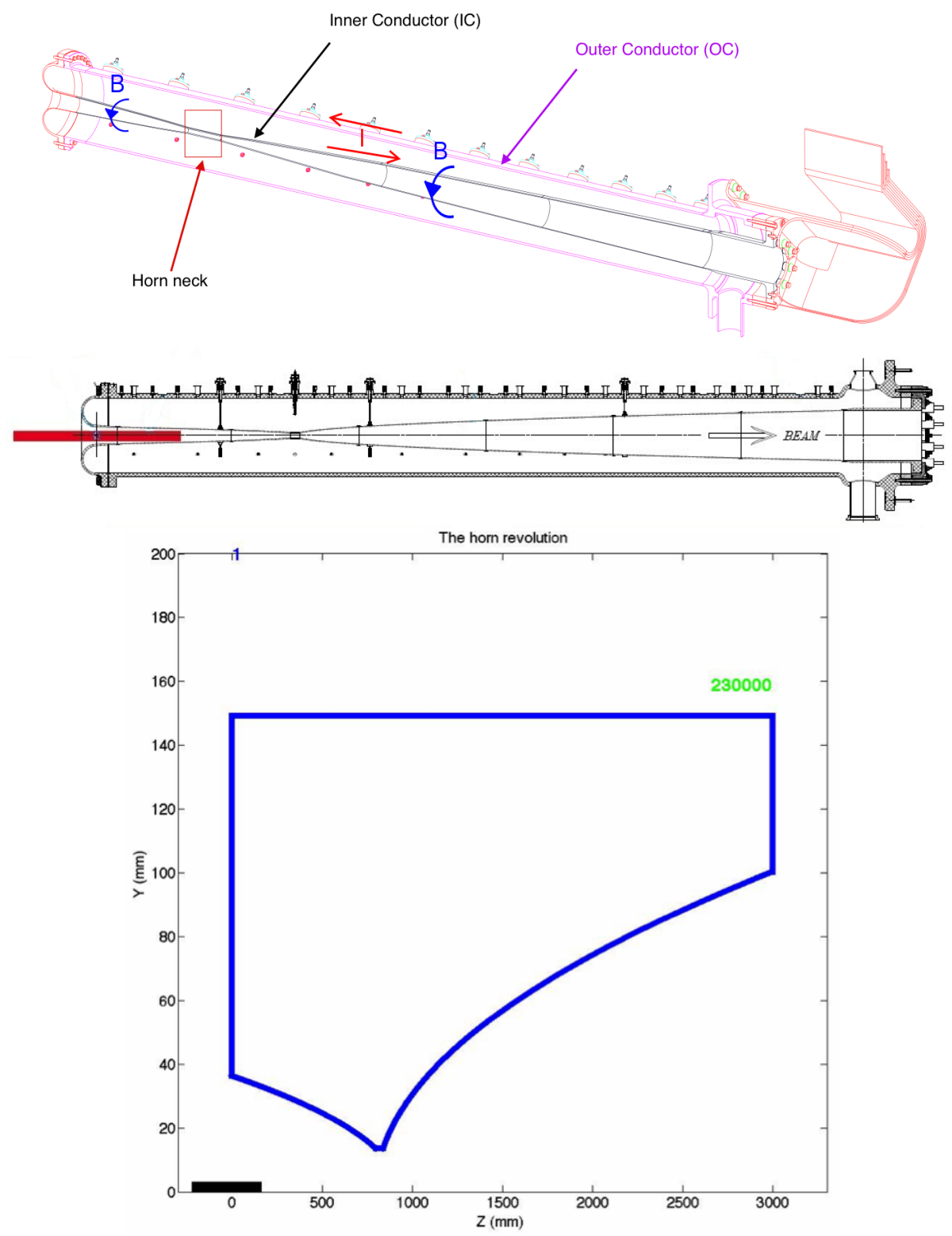

Figure 2.5: The schematic drawing of the NuMI horn 1 (upper) and LBNE horn 1 (middle). Both of the horns have a parabolic Inner Conductor (IC) for the sections before and after the neck. The schematic drawing of the nuSTORM baseline target and horn (bottom) shows the actual size of these target station elements. The nuSTORM horn runs at a peak current of $230 \mathrm{kA}$. 


\subsubsection{Introduction to the simulation tools}

Most of the beamline lattice D\&S is done in two parts: beam optics design and particle tracking. MAD-X is used for the nuSTORM optics design. It is also the software used for optics drawing in this thesis. MAD has been serving as one of the most frequently used tools at the forefront of computational physics in the field of particle accelerator design and simulation $[21,22]$. The MAD scripting language is widely recognized as a standard to describe particle accelerators, simulate beam dynamics and optimize beam optics. MAD-X is the successor of an older MAD version, MAD8. It includes the Polymorphic Tracking Code (PTC) written by E. Forest, which is frequently used for tracking purposes [23-25]. The MAD-X PTC tracking is symplectic so that it preserves the phase space volume obeying Liouville's theorem.

G4Beamline (G4BL) is used for the nuSTORM particle tracking when particle decay processes are needed. It is also used for the visualization of the beamline in this thesis. G4BL is a software based on Geant4, which is a computer code toolkit designed for simulating the passage of particles through matter [26]. Compared to Geant4, G4BL is optimized for simulating beamlines, especially when stochastic processes are needed in the tracking. Typical cases are particle tracking for experiments such as MICE, g-2, mu2e, etc. It is also useful in simulating the radiation doses, due to particle interactions with matter. For example it is very helpful in simulating the muons passing through a block of particle degrader with solenoidal field around

the iron, and examining the phase space of the residual muons. It is one of the most frequently used simulation tools in the muon community [27,28], and is being imported to other studies for general particle tracking, for example in $[29,30]$. 
Besides the tools for the optics and tracking D\&S, a number of codes in MATLAB and Python were used in the nuSTORM simulation and optimization. Specifically, mpi4py [31] and numpy [32] are used in the Python optimization codes discussed in Chapter 4 and Chapter 5. An optimization toolkit called "pyOPTmpi" was developed to adopt the MPI-implemented mpi4py module and integrate the MPI with selected optimization algorithms. The toolkit was tested in a number of selected optimization problems, and the optimization studies in this thesis were run on NERSC's [33] Edison system in the Portable Batch Scheduling (PBS) job assignment environment. The usage of MPI and NERSC shows obvious improvement in the computing speed. The details of the optimization toolkit are reviewed in Chapter 4 and Chapter 5. 
Horizontal Phase Space of $\pi+$ after the NuMI-like Horn Collection Fitted by covariance matrix (red) and iterative Gauss-Newton method (green)

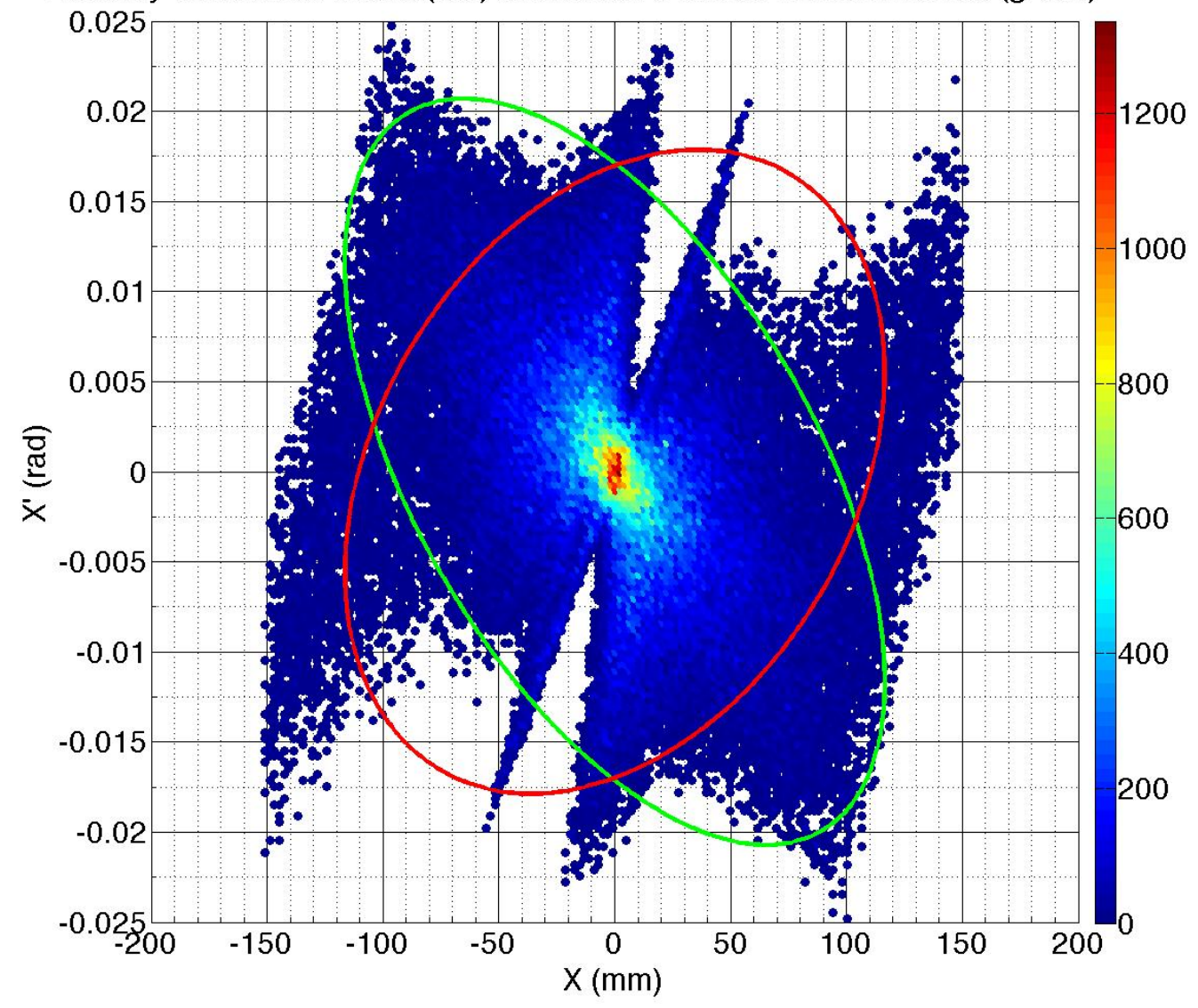

Figure 2.6: The phase space distribution of pions at the downstream end of the nuSTORM baseline horn. The simulation was done in MARS15 [20] and the numbers correspond to $2.4 \times 10^{7} 120 \mathrm{GeV}$ POT. The meaning of the green and red ellipses will be explained later in Section 3.4. 


\section{CHAPTER 3}

\section{PION BEAMLINE D\&S FOR nuSTORM}

One of the novel aspects of the nuSTORM facility design is the lack of a kicker system. The neutrino beams are obtained from muon decays in the production straight section of the FODO racetrack ring. However, it is difficult to create enough muons with the intensity that is needed directly from bombarding a target. In a conventional NF $[34,35]$ or even muon collider [36] scenarios, a medium to high $Z$ target is used to produce pions. The pions decay to muons, and the muons are then cooled to reduce the emittance, accelerated again by $\mathrm{RF}$ cavities, and collected by a beamline. The muons after collection are kicked into the ring and start circulating. This pion transport and muon collection beamline consists of at least a long decay section, a muon cooling section, an RF acceleration section, and a kicker system. This system requires not only devices with accurate timing control and current control, but also is quite long. Consequently, it would be much cheaper in cost if the whole system can be simplified.

In this chapter, a novel injection scenario, "stochastic injection" (initially proposed by D. Neuffer [37] in 1980) is introduced and discussed. A pion beamline that includes the stochastic injection is described that has a reference momentum $P_{\pi 0}=5 \mathrm{GeV} / \mathrm{c}$ to transport the pions from the target to the end of the production straight. It also 
accommodates the muons produced during the transport. This chapter also serves as a more detailed introduction to the general nuSTORM facility and beams.

\subsection{Definition of the pion beamline and stochastic injection}

As shown in Figure 2.4, the pions are propagated from the target and through the horn. They pass through the beam transport line, the injection section, a group of FODO cells, and are finally extracted by a mirror of the injection section. The pion beamline is defined as the complete path that the reference pion passes through before extraction.

The stochastic injection avoids the use of a fast muon beam kicker, but uses a transition part of the ring from a dispersive area to a non-dispersive area to combine the beam path of the injected pions with the beam path of circulating muons. The injection section of nuSTORM is named the "Orbit Combination Section (OCS)" due to this feature. Once the pion orbits are combined non-dispersively with the muon orbits in the ring straight section, the pions start regular betatron motion in the production straight and decay to muons. Since the muons are already in the ring structure, beam kickers are not needed.

Because the neutrinos mainly come from the production straight of the ring, which points directly at the detectors, the primary concern is to design it efficiently to produce a high neutrino yield. Therefore, the complete optics design of the pion beamline is done in the reverse beam direction, or namely from the production straight back to the horn. Specifically, the design process starts with the FODO structure (Section 3.2), is matched back to the OCS, and ends at the downstream face of the horn. It has to efficiently transport both pions and muons in the production straight, obtain enough beam separation at the injection section, and match the start and end 

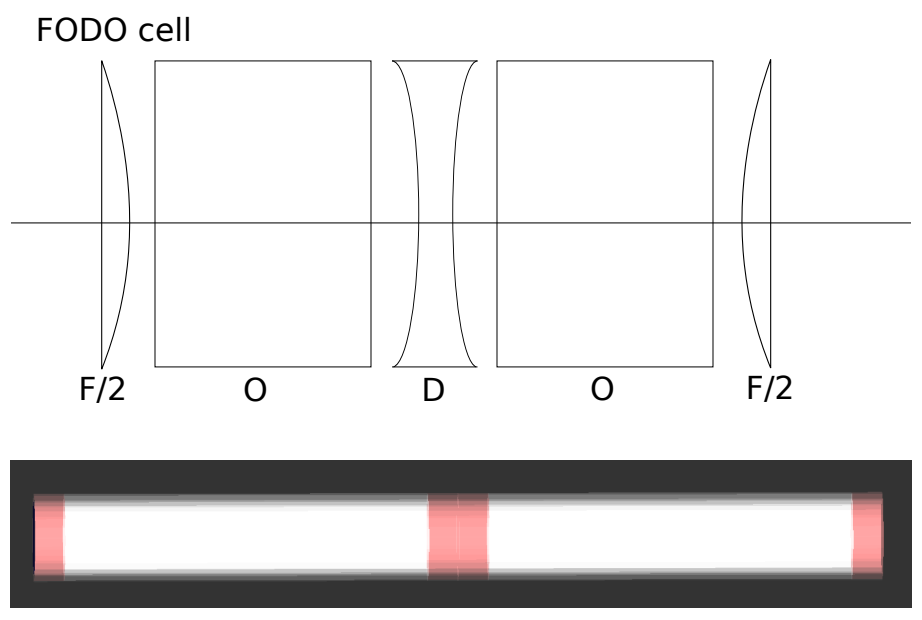

Figure 3.1: The schematic drawing of a FODO cell (upper) and the model in G4BL (lower). The quadrupoles are in red and the drift spaces are in white in the G4BL drawing.

to zero dispersion.

\subsection{FODO structure D\&S}

A FODO structure is frequently used in particle storage rings and transport beamlines. It is composed of a number of FODO cells that are formed by a pair of focusing and defocusing quadrupoles with either drift spaces or dipoles in between. A representation of a FODO cell can be written as

$$
\left\{\frac{1}{2} \mathrm{~F} \quad \mathrm{O} \quad \mathrm{D} \quad \mathrm{O} \quad \frac{1}{2} \mathrm{~F}\right\}
$$

where $\mathrm{O}$ is a drift space or a dipole. A schematic drawing of a FODO cell and its representation in G4Beamline (G4BL) are shown in Figure 3.1.

The transfer matrix of a FODO cell with $\mathrm{O}$ as drift spaces in the thin-lens ap- 
proximation is

$$
\mathbf{M}=\left(\begin{array}{cc}
1-\frac{L^{2}}{2 f^{2}} & 2 L\left(1+\frac{L}{2 f}\right) \\
-\frac{L}{2 f^{2}}\left(1-\frac{L}{2 f}\right) & 1-\frac{L^{2}}{2 f^{2}}
\end{array}\right)
$$

where $f$ is the focal length for the focusing quadrupole, and the defocusing quadrupole is assumed to have the same absolute focal length but with the opposite sign, $-f$. $L$ is the half cell length. The Twiss functions at the center of the focusing and defocusing quadrupoles are

$$
\begin{gathered}
\beta_{F}=\frac{2 L(1+\sin \Phi / 2)}{\sin \Phi}, \quad \beta_{D}=\frac{2 L(1-\sin \Phi / 2)}{\sin \Phi}, \alpha=0 \\
\cos \Phi=1-\frac{L^{2}}{2 f^{2}}
\end{gathered}
$$

\subsubsection{FODO cell design strategy}

The periodic Twiss functions for the same FODO cell (same quadrupole gradient $B_{1}$ ) change with different particle rigidities $B \rho$, since $f$ is a function of $K_{1}=B_{1} / B \rho$. The pions and muons with different momenta move simultaneously in the nuSTORM FODO structure, therefore, two sets of periodic Twiss functions of the two reference particle orbits need to be determined for the same configuration of one FODO structure.

The momentum $P_{\pi 0}$ of the reference pion was picked as a trade-off between the muon yield within $3.8 \pm 10 \% \mathrm{GeV} / \mathrm{c}$ and the dispersion needed at the OCS. For smaller $P_{\pi 0}$, higher muon yield can be expected. However, the dispersion $D_{x}$ required for combining the two reference orbits from a certain separation interval is also larger. Since the statistical horizontal beam size in a linear approximation is $\sqrt{D_{x}^{2} \delta^{2}+\epsilon_{x} \beta_{x}}$, a large $D_{x}$ is a disadvantage for the circulating muons as the beam size is greatly enlarged. Three $P_{\pi 0}, 4.5,5$, and $5.5 \mathrm{GeV} / \mathrm{c}$ were tested in the design. The effective muon yield is approximately $12 \%$ and $14 \%$ higher for the case where $P_{\pi 0}=5 \mathrm{GeV} / \mathrm{c}$ 
compared to the other two. This value was chosen as the reference momentum of the injected pion beam.

\subsubsection{Twiss functions for the two reference momenta}

The two sets of periodic Twiss functions for the $3.8 \mathrm{GeV} / \mathrm{c}$ reference muon and 5 $\mathrm{GeV} / \mathrm{c}$ reference pion can be determined in the following order:

- Scan a group of combinations of $\left(\beta_{F}, \beta_{D}\right)$ for the $3.8 \mathrm{GeV} / \mathrm{c}$ muon, and use MADX to find the exact FODO cell setup that gives each of the periodic solutions;

- Keeping the same setup for each $\beta$ combination, try to find the periodic solution for $\left(\beta_{\pi F}, \beta_{\pi D}\right)^{1}$ corresponding to the $5 \mathrm{GeV} / \mathrm{c}$ pion;

- In G4BL, construct the production straight FODO structure for each combination, which is approximately 150 meters. Count the number of cells needed for the straight section and start multi-particle tracking with 100,000 pions, which are Gaussian and uniformly distributed in the transverse and longitudinal directions.

- Use virtual detectors in G4BL to record and calculate the average angular divergence of the produced muon beam and the number of muons within $3.8 \pm 10 \%$ $\mathrm{GeV} / \mathrm{c}$ at the end of the production straight.

The three criteria in the determination are as follows. In order to collect more circulating muons in the decay ring, the number of muons within $3.8 \pm 10 \% \mathrm{GeV} / \mathrm{c}$ at the end of the production straight needs to be maximized; on the other hand, for the purpose of collecting more neutrinos at the detectors, the angular divergence of the

${ }^{1}$ The subscript $\pi$ is used to distinguish the $\beta$ functions of the pion from that of the muon. 
muon beam needs to be minimized, which can be characterized by the maximization of $1 / \sigma_{\theta}^{2}$. Last but not the least, the number of cells needed for the same length of production straight needs to be minimized to lower the facility cost.

It is hard to find a balance among the above criteria in a completely quantitative way because there is no direct analytical relationship between them. The absolute values of the above criteria for a scan of the $\left(\beta_{F}, \beta_{D}\right)$ of the reference muon are plotted in Figure 3.2. Notice that $\beta_{F}$ is always larger than $\beta_{D}$ in the scan, so only combinations in the lower triangle region were tested. The white blocks in the appropriate region represent combinations that cannot yield valid period Twiss solutions of a FODO cell with non-superconducting (or "conventional") quadrupoles. As a balanced solution for the three criteria, the FODO cell was set to be 7 meters long, with 0.5 $\mathrm{m}$ long conventional quadrupoles and $\left(\beta_{F}, \beta_{D}\right) \approx(30.2,23.3) \mathrm{m}$. The corresponding $\left(\beta_{\pi F}, \beta_{\pi D}\right) \approx(38.5,31.6) \mathrm{m}$. The periodic Twiss functions for 5 consecutive cells are plotted in Figure 3.3. The importance of using the different Twiss functions in the optics design is shown by illustrating the Twiss of the $5 \mathrm{GeV} / \mathrm{c}$ pion in 5 FODO cells in the figure, supposing the initial values were the same with those of the $3.8 \mathrm{GeV} / \mathrm{c}$ muon. The $\beta_{u}(u=x, y)$ quickly amplifies, which eventually would result in beam loss due to a large beam size.

\subsection{From the FODO to the OCS}

\subsubsection{Introduction}

The FODO structure in Section 3.2 was designed to have the trajectories of the reference pion and muon at the center of the structure, which indicates that the structure is non-dispersive. In a regular injection scenario of a storage ring, this is usually obtained by using a fast beam kicker to kick the beam onto the circulating 

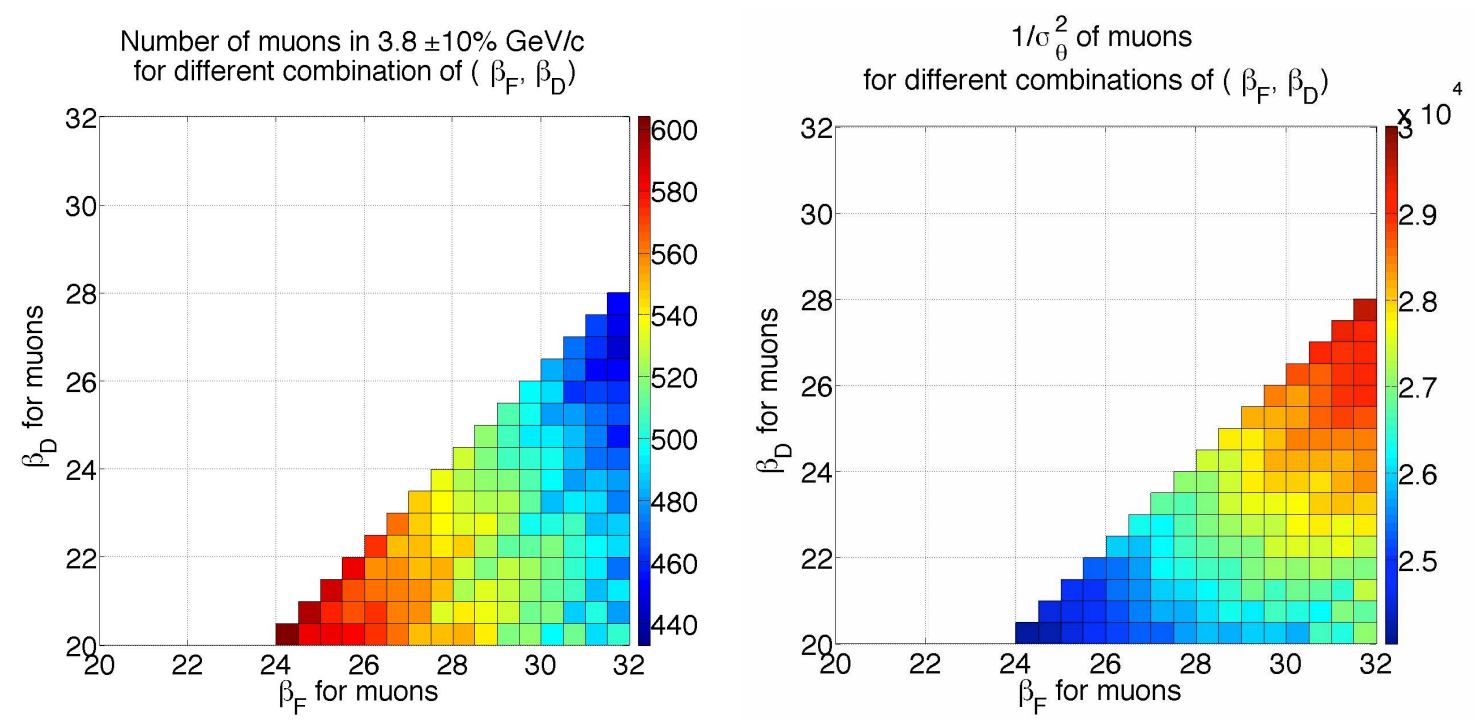

Number of FODO cells in $\sim 150 \mathrm{~m}$ production straight for different combinations of $\left(\beta_{F}, \beta_{D}\right)$

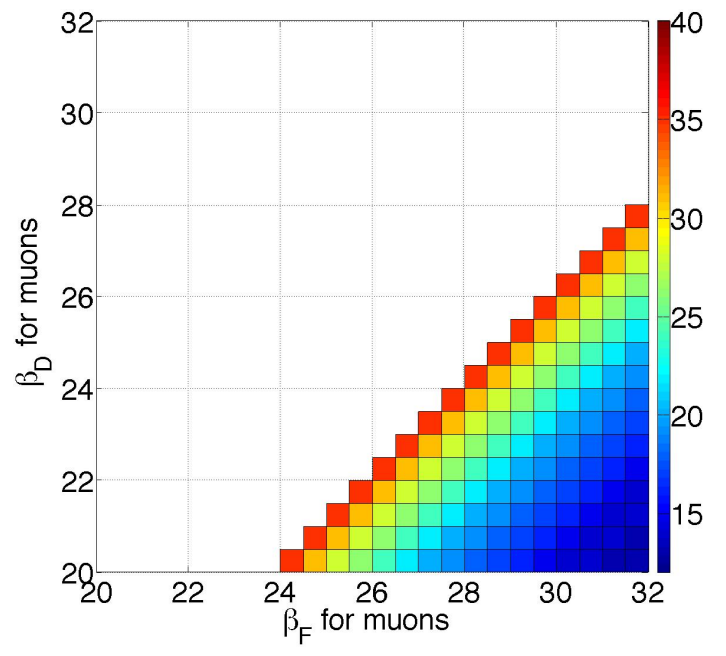

Figure 3.2: The absolute values of the three criteria. The smaller values are always represented in blue and the larger values are in red. The number of muons in $3.8 \pm 10 \%$ $\mathrm{GeV} / \mathrm{c}$ (upper left) and $1 / \sigma_{\theta}^{2}$ (upper right) are to be maximized, while the number of cells is to be minimized (lower). The three criteria favor (lower $\beta_{F}$, lower $\beta_{D}$ ), (higher $\beta_{F}$, higher $\beta_{D}$ ), (higher $\beta_{F}$, lower $\beta_{D}$ ), respectively. 

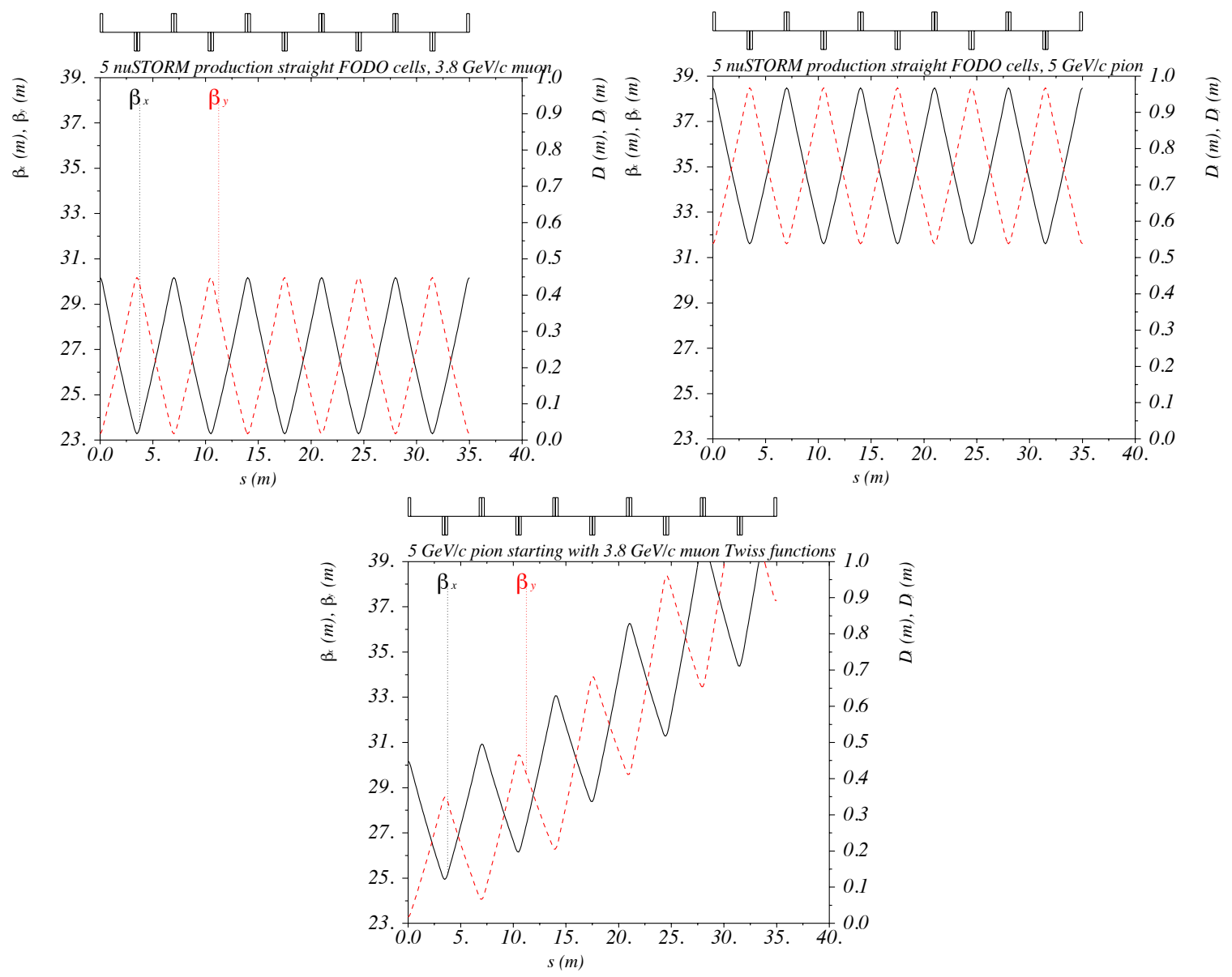

Figure 3.3: The periodic Twiss parameters for the two reference particles in the 5 nuSTORM production straight FODO cells (upper left - $3.8 \mathrm{GeV} / \mathrm{c}$ muon; upper right - $5 \mathrm{GeV} / \mathrm{c}$ pion). The setup of the FODO cell is the same for both left and right. As a comparison, the Twiss parameters for $5 \mathrm{GeV} / \mathrm{c}$ pion in the 5 cells starting from the same values with the muon is shown in the lower plot. The $\beta_{u}(u=x, y)$ quickly jumps to very large values that cause an excessively large beam size. 
orbit. A fast beam kicker for the nuSTORM muon beam with a beam size on the scale of $20 \mathrm{~cm}$ is pushing the state-of-the-art.

Matching backward toward the arc, it is possible to create a dispersive area starting from the very beginning of the FODO structure with enough separation between the two reference orbits at a location to be used as the injection point. The beam pipe is also expected to divide at the injection point, into a separate pion beamline and the muon decay ring. Given a $5 \mathrm{GeV} / \mathrm{c}$ pion starting off from the center of the FODO structure, the position and angle of the pion in the local phase space coordinates of the muon can be calculated. Since the particle trajectory in a beamline is reversible, the orbit of the reference pion when being injected is at the same location but with the direction reversed. It will be guided to the center of the decay ring and combined with the orbit of the reference muon. All the magnets used in the injection can be fed by DC current, thus use of a fast kicker is avoided. The dispersion is created only horizontally in order to place the whole beamline at the same elevation. Thus the injection will be done horizontally with the dispersion created in $x, D_{x}$.

The section in which the reference pion and muon orbits are combined is referred to as the Orbit Combination Section (OCS). A schematic drawing of the OCS is shown in Figure 3.4, where the OCS is enclosed in the red rectangle.

\subsubsection{Design procedure}

The design was done in the reverse direction of the injected or circulating beams. From the transfer matrices presented in Equation (1.32), dispersion can be created from a non-dispersive area using a dipole. The dispersion will be amplified if the 


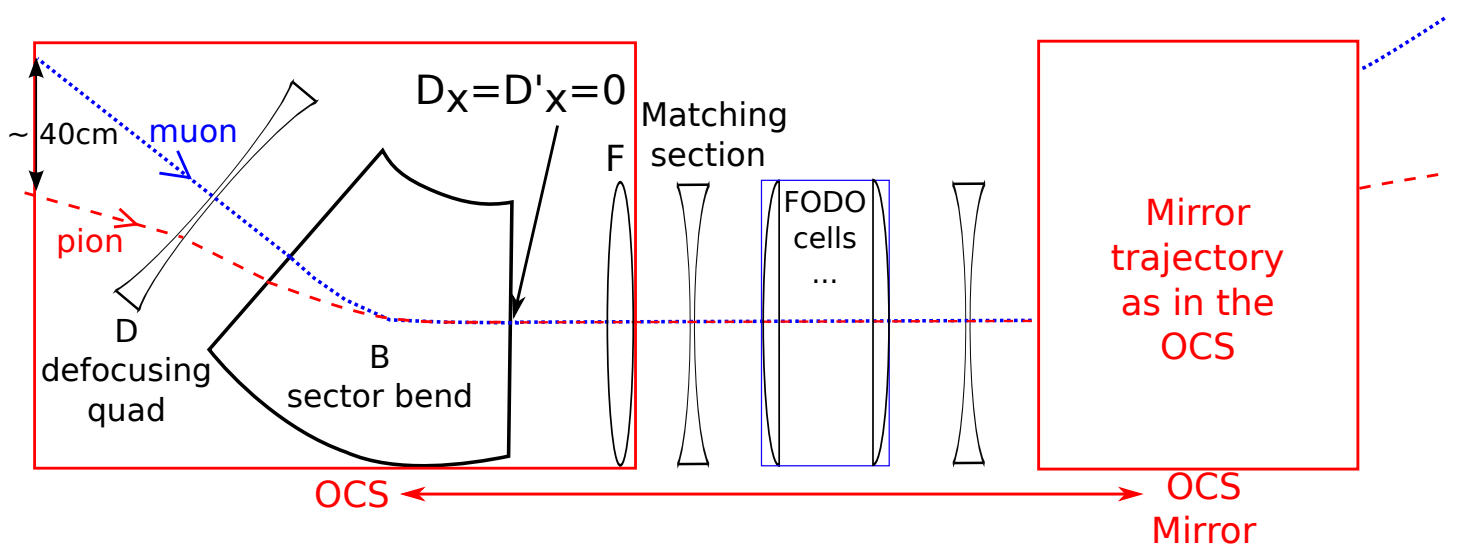

Figure 3.4: The schematic drawing of the OCS. The beams move left-to-right.

dipole is followed by a defocusing quadrupole:

$$
\begin{aligned}
\left(\begin{array}{c}
D_{1} \\
D_{1}^{\prime} \\
1
\end{array}\right)= & \left(\begin{array}{ccc}
\cosh \sqrt{-K_{D}} l_{D} & \frac{1}{\sqrt{-K_{D}}} \sinh \sqrt{-K_{D}} l_{D} & 0 \\
\sqrt{-K_{D}} \sinh \sqrt{-K_{D}} l_{D} & \cosh \sqrt{-K_{D}} l_{D} & 0 \\
0 & 0 & 1
\end{array}\right)\left(\begin{array}{ccc}
1 & l_{O} & 0 \\
0 & 1 & 0 \\
0 & 0 & 1
\end{array}\right) \cdots \\
& \ldots\left(\begin{array}{ccc}
\cos \theta & \rho \sin \theta & \rho(1-\cos \theta) \\
-(1 / \rho) \sin \theta & \cos \theta & \sin \theta \\
0 & 0 & 1
\end{array}\right)\left(\begin{array}{l}
0 \\
0 \\
1
\end{array}\right) \\
= & \left(\begin{array}{c}
\cosh \sqrt{-K_{D}} l_{D}\left(\rho(1-\cos \theta)+l_{O} \sin \theta\right)+\frac{\sin \theta}{\sqrt{-K_{D}}} \sinh \sqrt{-K_{D}} l_{D} \\
\sqrt{-K_{D}} \sinh \sqrt{-K_{D}} l_{D}\left(\rho(1-\cos \theta)+l_{O} \sin \theta\right)+\cosh \sqrt{-K_{D}} l_{D} \sin \theta \\
1
\end{array}\right)
\end{aligned}
$$

where $K_{D}$ and $l_{D}$ are the effective gradient and the length of the defocusing quadrupole, $\rho$ and $\theta$ are the bending radius and bending angle of the sector dipole. $l_{O}$ is the drift space length in between the two magnets. With a positive $D_{1}^{\prime}$, the dispersion will be 
larger after another drift space that is $l_{O 2}$ long.

$$
\left(\begin{array}{c}
D_{2} \\
D_{2}^{\prime} \\
1
\end{array}\right)=\left(\begin{array}{c}
\left(\cosh \sqrt{-K_{D}} l_{D}+l_{O 2} \sqrt{-K_{D}} \sinh \sqrt{-K_{D}} l_{D}\right)\left(\rho(1-\cos \theta)+l_{O} \sin \theta\right)+ \\
\sin \theta\left(\frac{\sinh \sqrt{-K_{D}} l_{D}}{\sqrt{-K_{D}}}+l_{O 2} \cosh \sqrt{-K_{D}} l_{D}\right) \\
\sqrt{-K_{D}} \sinh \sqrt{-K_{D}} l_{D}\left(\rho(1-\cos \theta)+l_{O} \sin \theta\right)+\cosh \sqrt{-K_{D}} l_{D} \sin \theta \\
1
\end{array}\right)
$$

The other requirement for the OCS is that the $\beta$ functions need to be reduced while $D_{x}$ is created. The optics of the $3.8 \mathrm{GeV} / \mathrm{c}$ reference muon from the FODO structure to the injection point is shown in Figure 3.5. The OCS red rectangle corresponds to the same one shown in Figure 3.4.

If the tracking is started simultaneously at the center of the magnets with $p_{x}=$ $p_{y}=0$ for a $3.8 \mathrm{GeV} / \mathrm{c}$ muon and a $5 \mathrm{GeV} / \mathrm{c}$ pion, and transported left-to-right in the section, shown in Figure 3.5, the two orbits will start to deviate at the beginning of the sector dipole (B).

In order to find the optics for the $5 \mathrm{GeV} / \mathrm{c}$ pion in the same section, the actual deviation and angle of the pion in the local phase-space frame of the muon at each position $s$ are needed. This can be done by using the MADX PTC_TRACK module or with G4BL tracking. Tracking, rather than direct calculation using the product of $\delta=\Delta P / P_{0}$ with $D_{x}$ in Equation (3.5), is preferred because $D_{x}$ was obtained with a linear approximation in $\delta$. For $\delta=(5-3.8) / 3.8 \sim 0.32$, the higher order terms in $\delta$ are significant. Results from the two tracking methods are compared to ensure compatibility. The schematic drawing of the two orbits from the downstream end of the dipole to the injection point is shown in Figure 3.6.

The horizontal coordinate and angular divergence of the reference pion are specifically recorded at three points $\mathbf{a}, \mathbf{b}, \mathbf{c}$ (marked in red) in Figure 3.6. The coordinates in both MADX and G4BL are measured with respect to the ideal orbit, which is the 


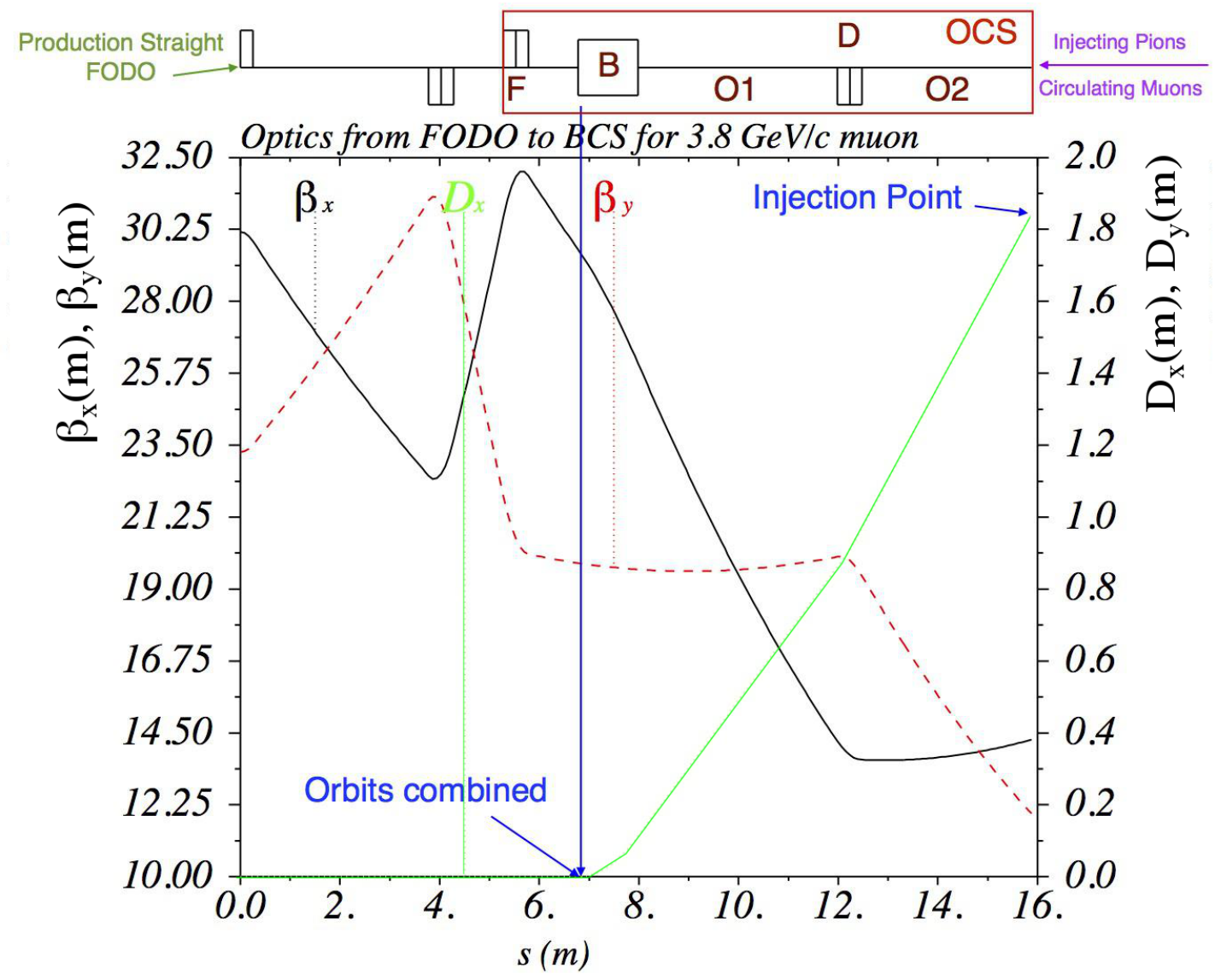

Figure 3.5: The optics of the $3.8 \mathrm{GeV} / \mathrm{c}$ reference muon from the FODO structure to the injection point. Both the injected pions and circulating muons move right-to-left. $\beta_{x}$ : black, $\beta_{y}$ : red, $D_{x}$ : green. 


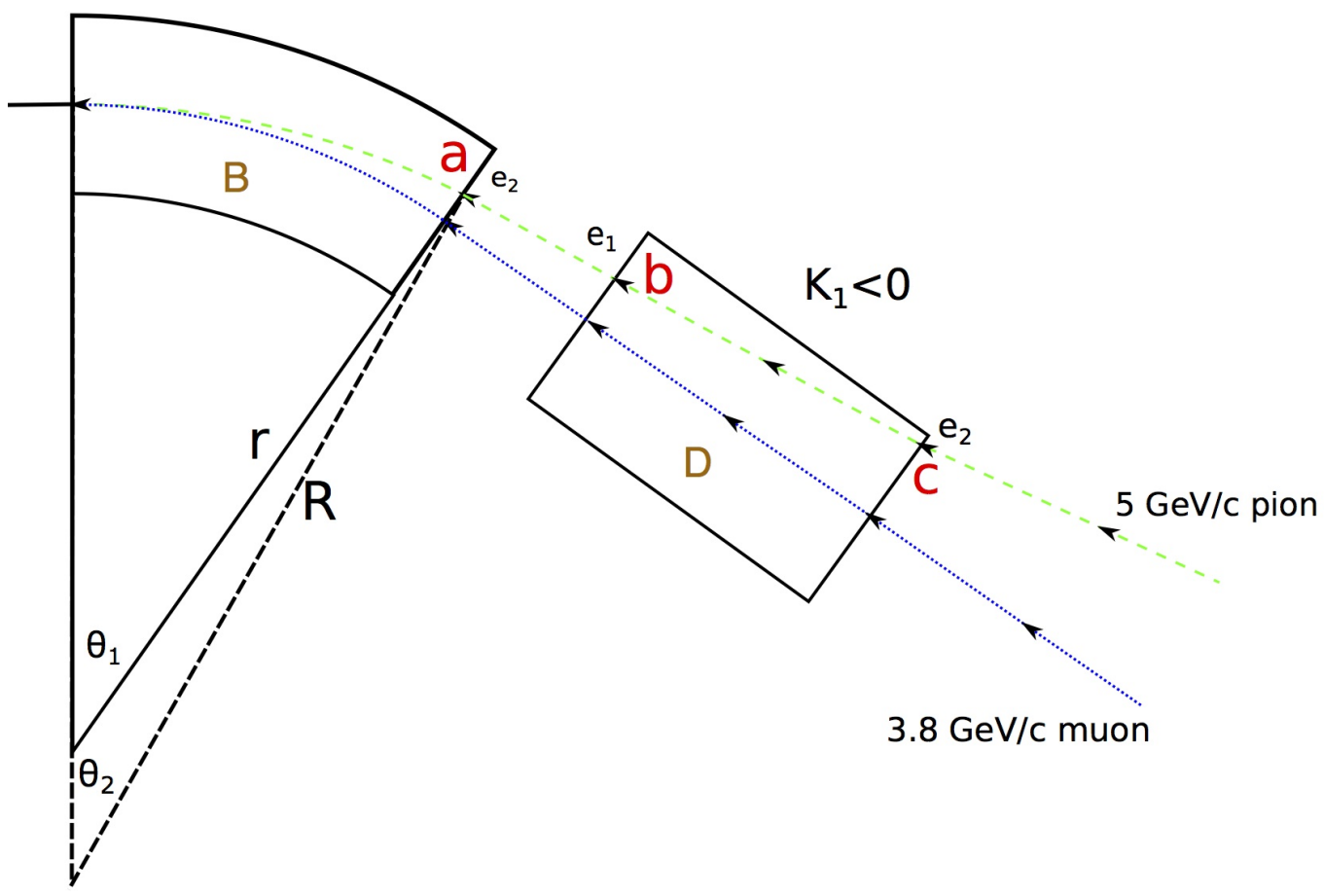

Figure 3.6: The schematic drawing of the two orbits from the downstream end of the dipole to the injection point. The specific points of interest are marked in red, where the pion orbit is tracked and recorded to obtain the correct optics. 
orbit of the reference muon in this case. The angle is measured in $P_{X}$ in MADX, which is the canonical momentum $p_{x}$ divided by the ideal momentum $p_{0}$, while it is measured in $p_{x}$ and $p_{z}$ in the local reference frame of the muon in G4BL. A pure sector dipole, B, for the muon transport has an effective exiting pole-face rotation angle $e_{2}$ for the pion at a. Similarly, the pure defocusing quadrupole, D, for the muon becomes a combined function dipole for the pion, which has both the entering and the exiting pole-face rotation angles $e_{1}$ and $e_{2}$ at $\mathbf{b}$ and $\mathbf{c}$, respectively. The sign convention of the pole-face rotation angles can be found in Appendix A.5. The corresponding reference pion optics in the same section after the above details are considered is given in Figure 3.7.

\subsection{From the OCS to the horn}

\subsubsection{Introduction}

After the reference pion optics is found for the OCS, the rest of the pion beamline is designed to satisfy two requirements. First, the beam optics at the injection point must be matched to the values at the downstream end of the horn (This includes both dispersion suppression and the matching of Twiss functions). Second, the suppression of the dispersion and its derivative $\left(D_{x}\right.$ and $\left.D_{x}^{\prime}\right)$ requires a bending magnet somewhere downstream of the straight section of the collection beamline after the horn. Viewed in the direction of the pion beam, it is the first bending magnet the beam encounters after leaving the horn (referred as the "first bend" for the rest of the thesis). The remaining primary $120 \mathrm{GeV}$ protons in the beam will be significantly less affected by the bending

field compared with the pion beam and continue practically straight thereafter. At a sufficient length after the first bend, the two beams can be further separated from each other with a stronger bending field. When the beams are separated enough 


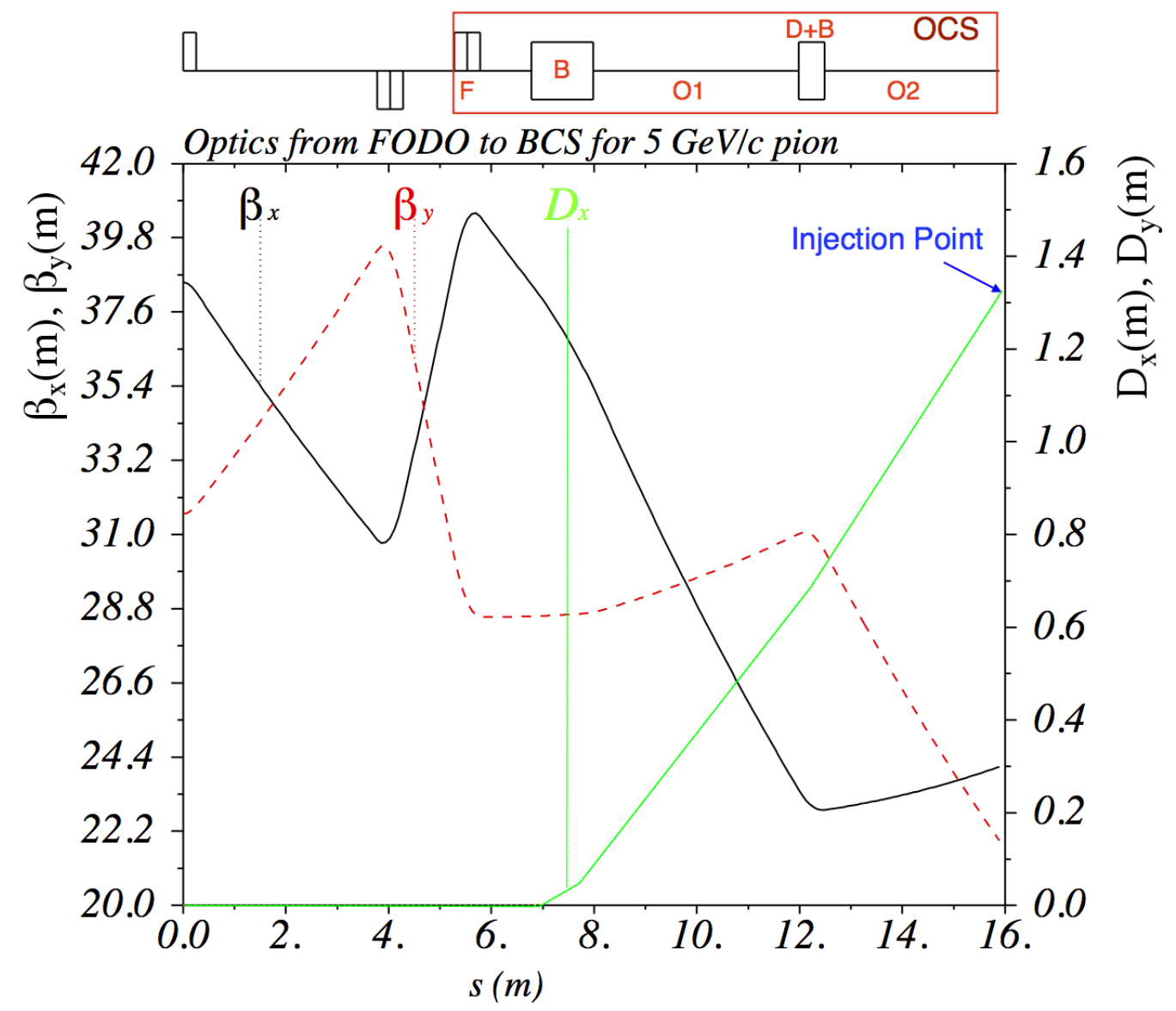

Figure 3.7: The corresponding reference pion optics from the FODO structure to the injection point. The magnets have been changed in MADX to match the $5 \mathrm{GeV} / \mathrm{c}$ pion's reference orbit. $\beta_{x}$ : black, $\beta_{y}$ : red, $D_{x}$ : green. 
from each other, the protons are directed onto a beam absorber in which they will be dissipated. In this section, the design of this initial part of the pion beamline will be discussed.

\subsubsection{Twiss functions of pions after the horn}

The Twiss functions of the pion beam at the downstream end of the horn can be found by fitting the transverse phase space distribution to a bivariate Gaussian Probability Distribution Function (PDF). The PDF is assumed to be the same for both vertical and horizontal directions following the azimuthal symmetry of the target and horn configurations.

As demonstrated by the example in Figure 3.11, the pions after the horn have a very wide angular divergence and momentum spectrum, which makes the transverse phase space diluted and distorted from a regular bivariate Gaussian distribution. In order to find more accurate Twiss functions from the distribution, a new fitting algorithm is required. A standard method of fitting data to a certain distribution probability function, the Gauss-Newton (GN) algorithm, is chosen to be the baseline of the new algorithm. The GN algorithm is a powerful tool to solve non-linear least squares problems, and can be used to minimize a sum of squared function values. As a result, the algorithm is widely used in data fitting in order to find the minimum summed squared residual values from the difference between the model and observation. More details of the algorithm are found in Appendix A.1.

Consider this secondary particle beam, for which the action of the $i$ th particle is defined by $J_{i}=\gamma u_{i}^{2}+2 \alpha u_{i} u_{i}^{\prime}+\beta u_{i}^{\prime 2}$, where $\alpha, \beta, \gamma$ are the Twiss parameters. Most of the particles with the largest action can not be accepted by the beamline. The bias in the fitting also comes mostly from the particles with the largest action, which 
means most of the particles are not matched by the fit. Straightforwardly obtaining the Twiss functions from the covariance matrix or a one-time GN fitting does not represent the core part of the beam, which is more likely to be accepted by the beamline and thus needs to be matched to the beamline.

It was realized that a new algorithm needs to take the above effect into account. Therefore, the Iterative GN (IGN) algorithm was developed to adapt the GN algorithm to the matching. This has an additional process that iteratively removes particles with the largest action. In the $n$th iteration, the GN algorithm is applied first to obtain the most accurate Twiss functions, $\alpha_{n}, \beta_{n}, \gamma_{n}$ for the current beam. Then the admittance $\Omega_{a d}$, defined conventionally by $\Omega_{a d}=6 \Omega_{r m s}$, of the current beam is calculated. If the $\Omega_{a d}$ is larger than the acceptance $\Omega_{\epsilon}$ of the beamline, the particles are then sorted by their action and a certain portion of the particles with the largest action are removed for the next iteration. The iterative adjustment shrinks the phase space area and is repeated until the beam satisfies $\Omega_{a d} \leq \Omega_{r m s}$. The GN fitting is then applied for the last time to obtain the final parameters. An illustrative flowchart of the IGN algorithm is shown in Figure 3.8.

\subsubsection{Dispersion Suppression and matching to the horn}

Two bending magnets with opposite-sign $\left(B_{y}\right)$ dipole field directions are used to suppress the dispersion functions $D_{x}$ and $D_{x}^{\prime}$. Quadrupoles are also used to control the Twiss and dispersion functions in the dispersion suppression. The capture quad channel between the horn and the $1^{\text {st }}$ dipole downstream the horn is required to have a length of at least 20 meters to avoid excessive radiation activation at the first bending magnet and civil construction. The capture channel matches the Twiss parameters exactly to the values obtained by the IGN fitting after the horn. Since the optics are 


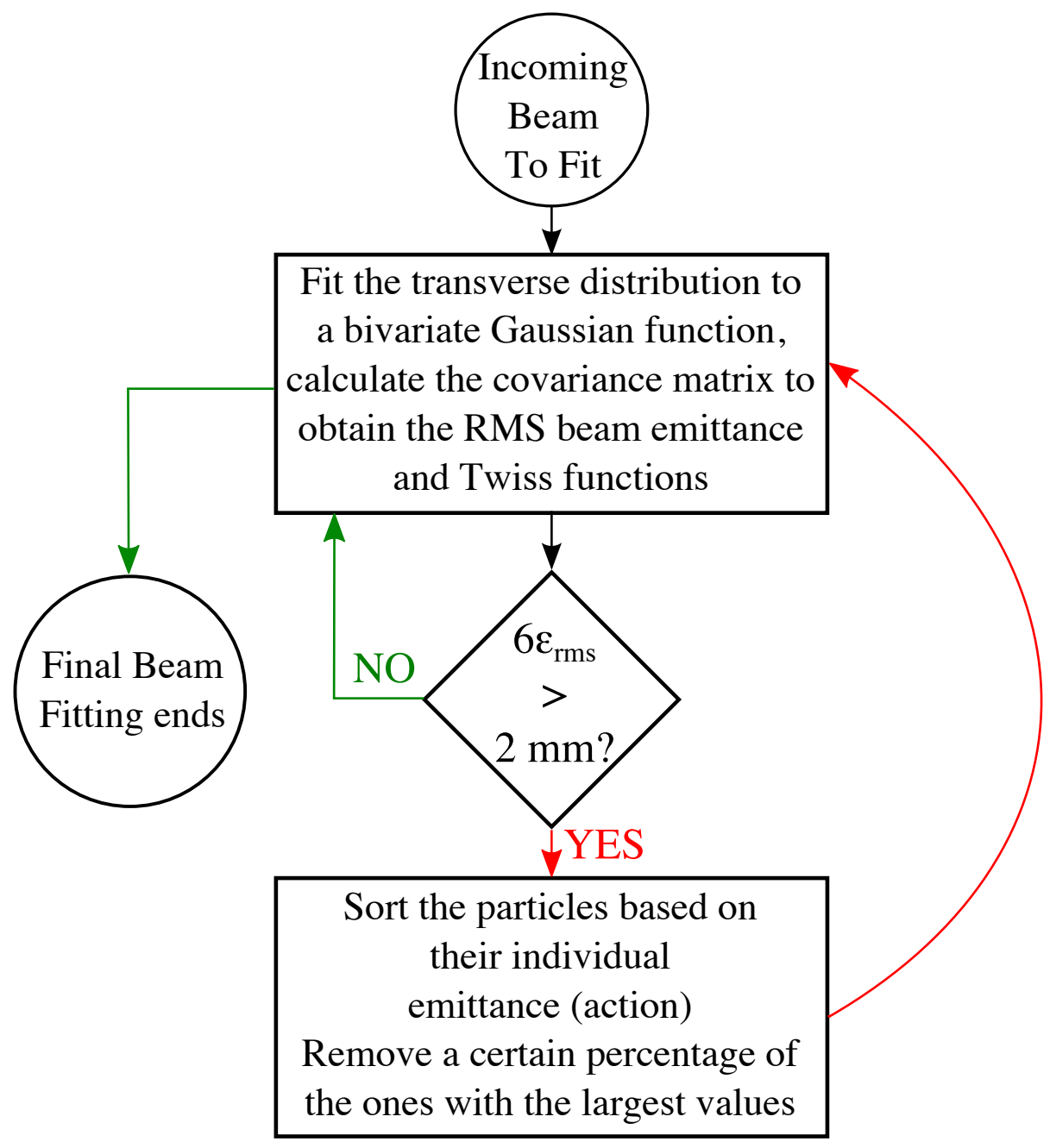

Figure 3.8: The IGN flowchart of the Iterative Gauss-Newton (IGN) fitting algorithm. An example of its application to fitting the transverse phase space distribution at the downstream end of the NuMI-like nuSTORM baseline horn is shown in Figure 2.6. The IGN algorithm (green) can better preserve the core of the phase space than the fitting using a covariance matrix method. 


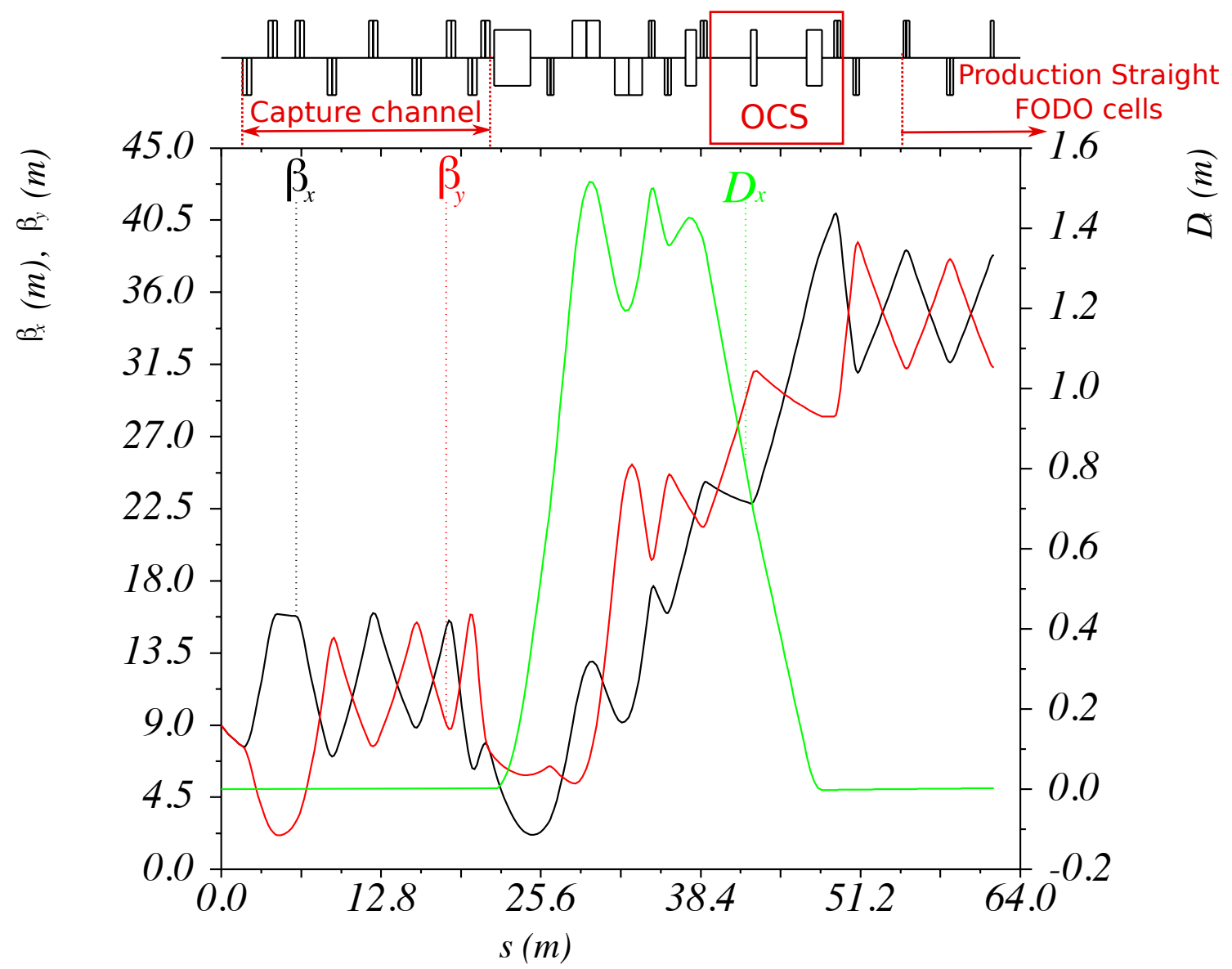

Figure 3.9: The pion beamline optics plotted in the pion beam direction, from the downstream end of the horn to the start of the FODO structure. The location of OCS is highlighted by the red block. The entire production straight section is represented by a single FODO cell to avoid repetitive plotting of the Twiss functions. The pions move from the left to the right in the drawing. $\beta_{x}$ : black, $\beta_{y}$ : red, $D_{x}$ : green. 
designed in the opposite direction to the beam flow, the sign of $\alpha=-\beta^{\prime} / 2$ obtained by the fitting needs to be reversed in the matching. After the matching, the beamline is reversed in MAD-X. The pion beamline optics in the direction of the beam flow is plotted in Figure 3.9, where the OCS is highlighted in the red box. The periodic structure of the FODO cells in the production straight is represented by one cell to avoid repeating Twiss data. The production straight contains 21 cells, with a total length of $\sim 150$ meters. The length of the pion beamline, including the production straight, is $\sim 200$ meters. In this length approximately $50 \%$ of the pions at $5 \mathrm{GeV} / \mathrm{c}$ will decay to muons.

\subsection{Pion tracking in G4BL}

In order to check the effectiveness of the beamline design, pion tracking with stochastic decay processes must be done. In this case, the tracking program of G4BL is used since it integrates the Monte-Carlo processes in Geant4 with the beamline elements, including cavities, linear and nonlinear magnets, solenoids, absorbers, etc. It is straightforward to convert a standard beamline to the G4BL format. In order to improve the D\&S efficiency of not only the nuSTORM beamline but also other facilities, a Python code was developed to convert the MAD-X outputs to the G4BL input (See Appendix A.4).

Because of constraints on the magnet definitions in G4BL, a sector dipole can not have arbitrary entrance and exit angles with respect to the reference particle as it can in MAD-X. However, since the magnets and the trajectory of the reference pion are calculated precisely as shown in Figure 3.6, it is possible to use the G4BL regular dipole definitions $\mathbf{B}$ and $\mathbf{D}$ to model the combined function magnets and the pole-face rotation angles correctly in G4BL. The rotation and displacement of the 
magnets can be calculated based on the PTC_TRACK data of the reference pion. A screen shot of the pion beamline structure is shown in Figure 3.10 (only one cell of the entire FODO production straight is shown).

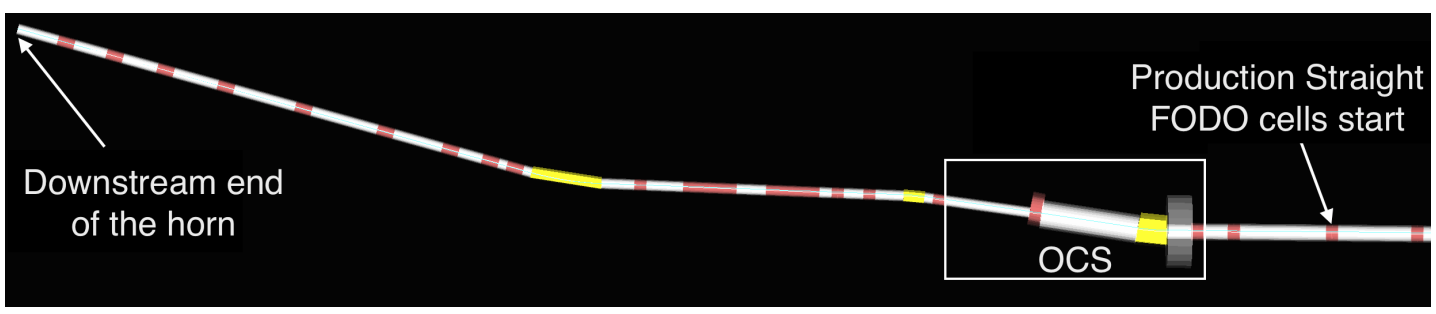

Figure 3.10: A screenshot of the pion beamline structure in G4BL display mode. The white, red and yellow blocks correspond to drift spaces, quadrupoles, and sector dipoles, respectively. The beam moves from the left to the right.

\subsubsection{The muons at the end of the production straight}

The success of the injection can be characterized by the number of muons within both the momentum acceptance $\Omega_{P}= \pm 10 \%$ and the phase space acceptance $\Omega_{\epsilon}=$ $2000 \mu \mathrm{m} \cdot \mathrm{rad}$ of the decay ring. The MARS-simulated pion beam generated from a 38 cm Inconel target and focused by a NuMI-like, 3 meters long nuSTORM baseline horn (see Figure 2.5) is used as the initial beam in the tracking. The MARS simulation was done with $2.4 \times 10^{7} 120 \mathrm{GeV}$ protons on target (POT) and imposed a radius cut of $15 \mathrm{~cm}$, considering the physical outer conductor (OC) limitation of the horn.

The aperture of vacuum chambers in the pion beamline is set to be $40 \mathrm{~cm}$-diameter $(\varnothing=40 \mathrm{~cm})$ for the beamline elements before the OCS. The OCS magnets and drift tubes have $\varnothing=1 \mathrm{~m}$ and for the remaining part of the beamline $\varnothing=60 \mathrm{~cm}$. The large aperture is required to contain the beam because there are no cooling devices in the facility design. 
The MARS simulation of the proton-target-horn interaction was first done using a horn with 0 Inner Conductor (IC) thickness ("simple horn"). This simplification was made based on the known low IC thickness of the horn, which is $4.5 \mathrm{~mm}$ for the NuMI horns. The number of pions in the momentum range of $(1 \pm 20 \%) P_{\pi 0}$ or $5 \pm 1$ $\mathrm{GeV} / \mathrm{c}$ is $\mathbf{0 . 2 9}$ per POT, within the full $r \leq 15 \mathrm{~cm}$ region. The $\mathrm{X}-\mathrm{X}$ ' phase space, $\mathrm{X}-\mathrm{Y}$ real space and momentum distributions are shown in Figure 3.11.

The muons are recorded at the end of the production straight. Because the decay kinematics of the pions give a wide range of muon momenta and angles, a momentum cut of $(1 \pm 10 \%) P_{\mu 0}$ or $3.8 \pm 0.38 \mathrm{GeV} / \mathrm{c}$ is applied to get the muons within the momentum acceptance of the decay ring. The IGN method (see Figure 3.8) is applied to obtain the Twiss parameters at the location. A particle action cut of $J_{i} \leq 2000 \mu \mathrm{m}$ is applied afterwards to obtain the number of useful muons within the full acceptance of the decay ring. A total of 0.05 muons per POT are found at the end of the production straight. With the $\Omega_{\epsilon}$ and $\Omega_{P}$ acceptance cuts, $\mathbf{0 . 0 1 3}$ muons per POT are found in the momentum and phase space acceptance of the ring. The distributions of muons at the end of the production straight before applying the cuts are shown in Figure 3.12. Part of the muons with higher momentum, specifically those within the $5 \pm 10 \% \mathrm{GeV} / \mathrm{c}$ range, can be extracted by an implementation of the OCS mirror at the end of the production straight, along with the pions that have not yet decayed. Extraction, just being the reversed process of the injection, is also stochastic.

\subsubsection{Possible contamination of the injected beam}

The dispersive section of the pion beamline created by the dipoles (see Figure 3.10) applies a momentum selection on the beam being transported. This is especially useful for the neutrino physics owing to the fact that the number of wrong-sign pions 

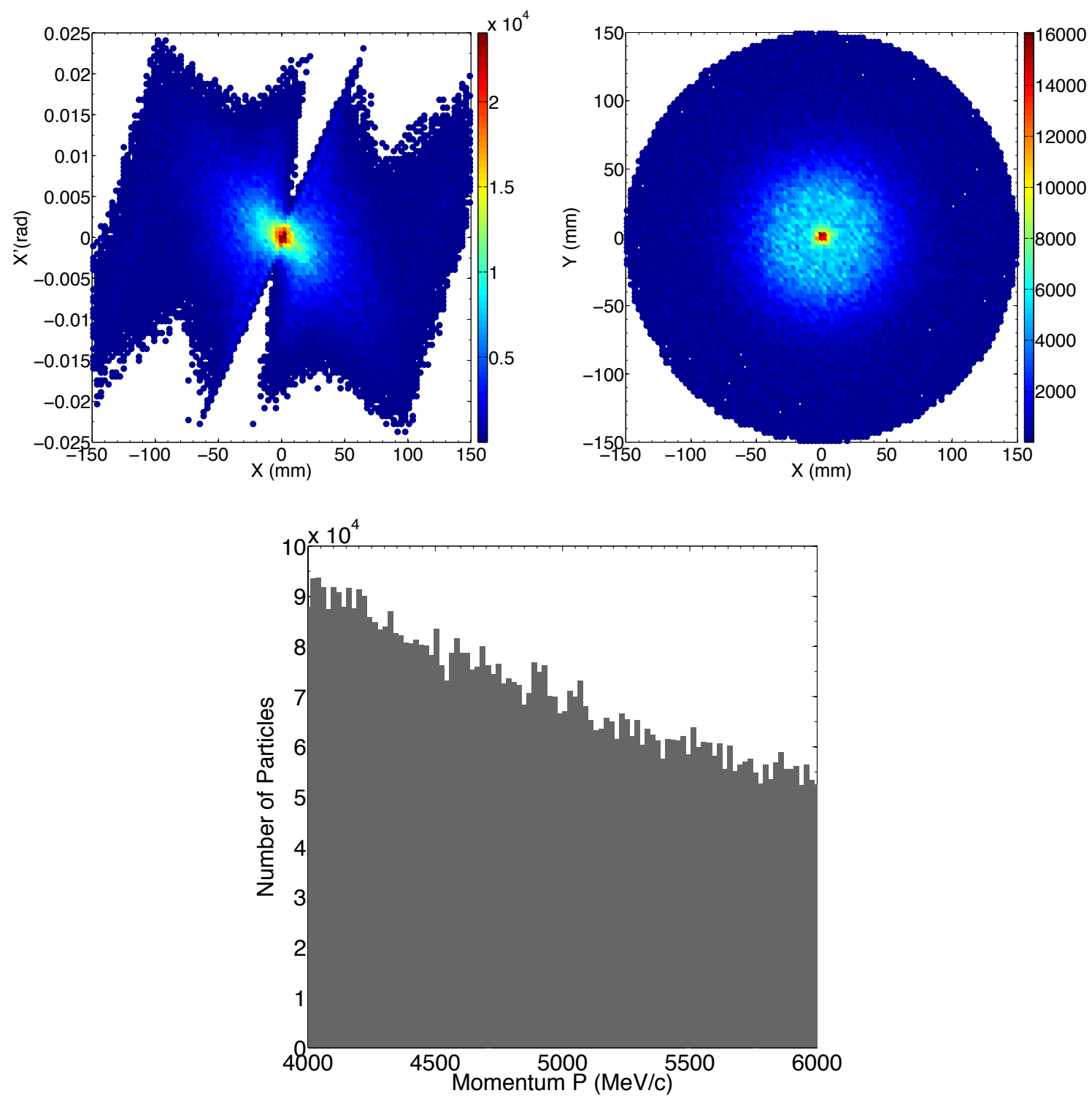

Figure 3.11: The $\mathrm{X}-\mathrm{X}^{\prime}$ phase space, $\mathrm{X}-\mathrm{Y}$ real space and momentum distribution of the pions after the "simple" nuSTORM baseline horn with a $38 \mathrm{~cm}$ Inconel target. The data corresponds to $2.4 \times 10^{7} 120 \mathrm{GeV}$ POT. The pion yield in $5 \pm 1 \mathrm{GeV} / \mathrm{c}$ and $r \leq 15 \mathrm{~cm}$ is 0.29 per POT, with no cuts on the particle actions $J_{i}$. 

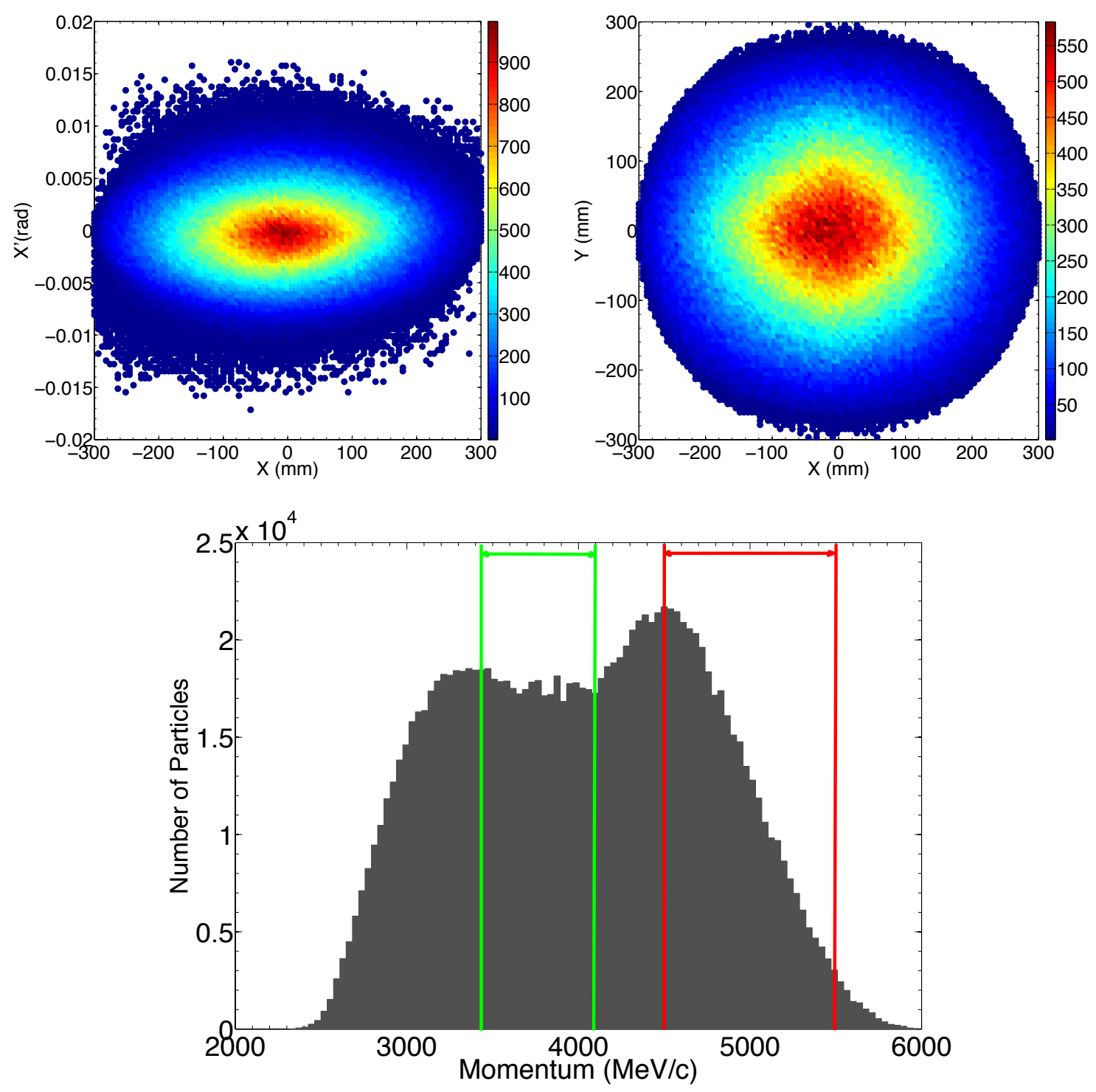

Figure 3.12: The phase space (upper left), real space (upper right) and momentum (lower) distributions of muons at the end of the production straight. The data corresponds to $2.4 \times 10^{7} 120 \mathrm{GeV}$ POT. The green $P$ band $(3.8 \pm 10 \% \mathrm{GeV} / \mathrm{c})$ is the momentum acceptance range of the decay ring, and the red band $(5 \pm 10 \% \mathrm{GeV} / \mathrm{c})$ is the OCS mirror extraction momentum range. 
or muons is greatly reduced after injection. However, the beamline can not eliminate the Kaons that are produced by the target and/or the horn conductor and are in the same momentum range as that of the pions. The Kaons have shorter natural lifetime and larger mass than the pions, thus will decay faster than the pions in the beamline. Therefore, in order to check the composition of the beam in the ring with possible contaminations from the wrong-sign particles and Kaons, particles of all-types are tracked through the pion beamline and analyzed.

The number of particles of each type off a $38 \mathrm{~cm}$ Inconel target bombarded by $2.4 \times 10^{7} 120 \mathrm{GeV}$ POT simulated in MARS is

Number of particles of each type within $5 \pm 1 \mathrm{GeV} / \mathrm{c}$, per POT:

$\pi+=0.481, \pi-=0.439, \mathbf{K}^{+}=0.059, \mathbf{K}^{-}=0.032$

The thickness of the horn IC (Aluminum, $4.5 \mathrm{~mm}$ thick) and the particle-matter interaction in the horn are modeled in MARS (closer to a "real horn") to obtain more accurate $\pi^{ \pm}$and $\mathbf{K}^{ \pm}$production rates. Compared with the "simple horn" mentioned in Section 3.5.1, the number of pions within $5 \pm 1 \mathrm{GeV} / \mathrm{c}$ at the downstream end of the "real horn" is increased by $4 \%$ to 0.32 per POT. However, the rms angular divergence of the pions is increased from $\sigma_{x^{\prime}}=6.2 \mathrm{mrad}$ to $16 \mathrm{mrad}$. The distribution of the pions after the "real horn" is shown in Figure 3.13.

The composition of the beam after the "real horn" is listed below. The horn is configured to focus positively charged particles. Since the wrong-sign particles are defocused in the horn, the ratio of the $\mathbf{K}^{-}$and $\pi-$ to $\pi+$ is already reduced by a factor of 10 .

Number of particles of each type within $5 \pm 1 \mathrm{GeV} / \mathrm{c}$, per POT:

$\pi+=0.317, \pi-=0.064, \mathbf{K}^{+}=0.031, \mathbf{K}^{-}=0.0034$ 

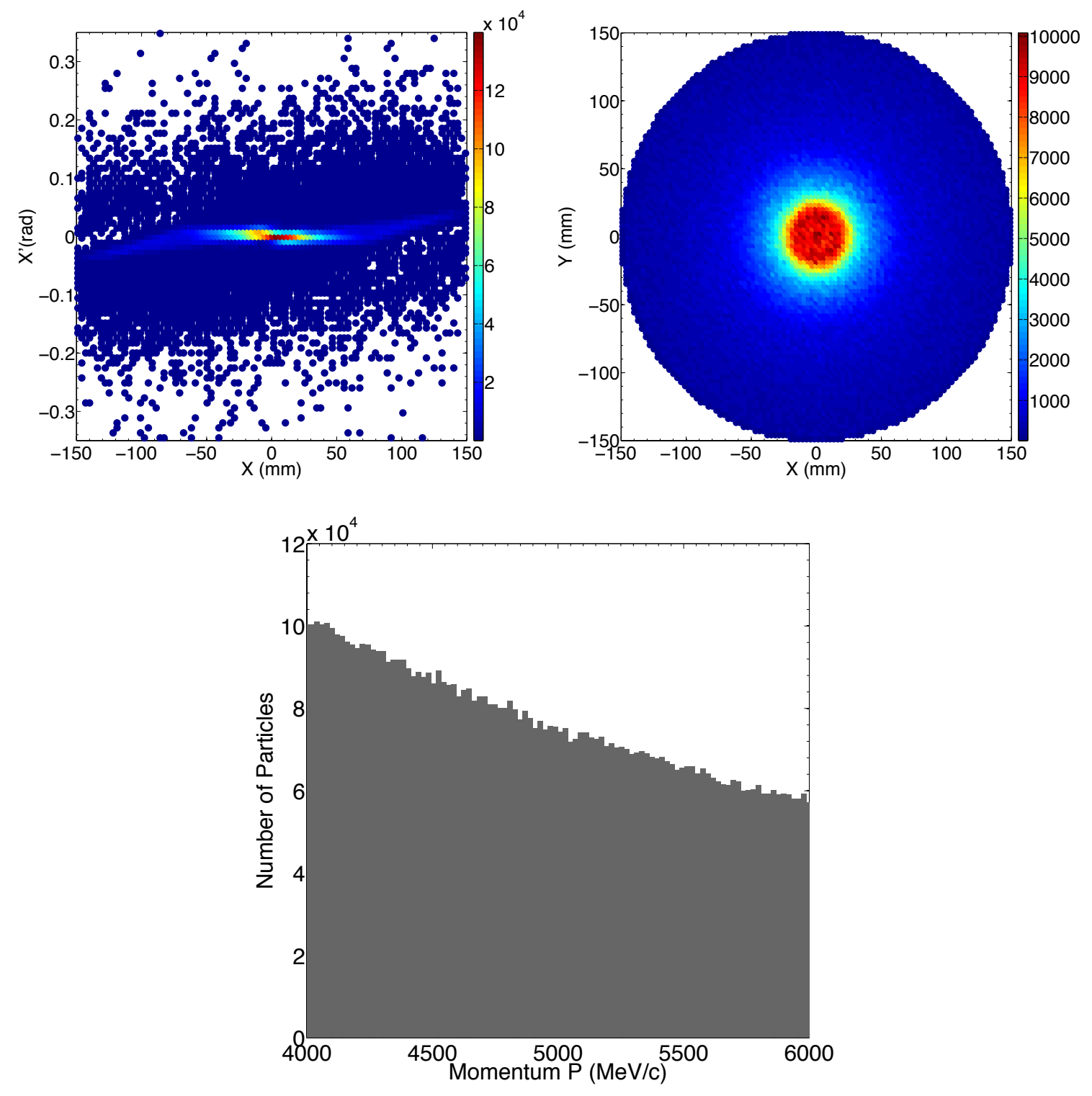

Figure 3.13: The $\mathrm{X}-\mathrm{X}$ ' phase space, $\mathrm{X}-\mathrm{Y}$ real space and momentum distributions of the pions after a "real" nuSTORM baseline horn with a $38 \mathrm{~cm}$ Inconel target. The data correspond to $2.4 \times 10^{7} 120 \mathrm{GeV}$ POT. The pion yield in $5 \pm 1 \mathrm{GeV} / \mathrm{c}$ and $r \leq 15$ $\mathrm{cm}$ is 0.32 per POT. 
All the necessary secondary particles are tracked in the pion beamline, with stochastic decays, and aperture limitations discussed in 3.5.1. The number of particles is recorded at two important locations, which are at the start and the end of the production straight section.

The number of particles of each type, at the beginning of the production straight, per POT:

$\pi+=0.10, \pi-=3.06 \times 10^{-5}, \mathbf{K}^{+}=2.55 \times 10^{-3}, \mathbf{K}^{-}=0, \mu+=0.011, \mu-=$ $1.32 \times 10^{-6}$

The number of particles of each type, at the end of the production straight, per POT:

$\pi+=0.056, \pi-=7.43 \times 10^{-6}, \mathbf{K}^{+}=4.91 \times 10^{-5}, \mathbf{K}^{-}=0, \mu+=0.041$, $\mu-=1.10 \times 10^{-5}$

In relative units with respect to the number of $\pi+$ at each location:

At the beginning of the production straight:

$\pi+=1, \pi-=3.06 \times 10^{-4}, \mathbf{K}^{+}=2.55 \times 10^{-2}, \mathbf{K}^{-}=0, \mu+=0.11, \mu-=1.32 \times 10^{-5}$ At the end of the production straight:

$\pi+=1, \pi-=1.33 \times 10^{-4}, \mathbf{K}^{+}=8.77 \times 10^{-4}, \mathbf{K}^{-}=0, \mu+=0.73, \mu-=1.96 \times 10^{-4}$ The number of $\mu+$ within both the $\Omega_{\epsilon}$ and the $\Omega_{P}$ acceptance of the ring is

0.011 per POT

It is shown by the above numbers that contamination from other particles other than $\pi+$ has been reduced by almost three orders of magnitude, measured as the ratio of the numbers of particles of each type to that of the $\pi+$. The number of useful muons drops by $15 \%$ with the "real horn" including the particle interactions with the horn conductor.

\subsection{Summary}

The pion beamline of nuSTORM has been successfully designed to transport the pions and the muons from pion decay from the horn to the end of the production straight 
of the muon decay ring. The dispersive region created by the dipoles to fulfill the stochastic injection eliminates the wrong-sign pions and kaons, while the correct-sign kaons decay fast enough that the percentage it occupies in the beam quickly drops in the pion beamline. Therefore, this feature of the nuSTORM facility helps to provide a better understood beam with much lower background at the detectors, compared with a conventional neutrino beam. From the particle tracking in magnetic fields and interactions with the nuSTORM baseline collection horn with $120 \mathrm{GeV}$ protons on a $38 \mathrm{~cm}$ Inconel target, 0.011 muons per POT are obtained within the $\Omega_{\epsilon}$ and $\Omega_{P}$ acceptance at the end of the production straight section. 


\section{CHAPTER 4}

\section{OPTIMIZATION OF THE MAGNETIC HORN FOR nuSTORM}

The use of a magnetic horn as a collection device was first proposed by van der Meer in 1961 [38] to focus pions forward in conventional neutrino experiments. Its success has been widely recognized, and it is now a standard element in neutrino experiments world-wide. It is capable of focusing secondary particles with a wide angular divergence and broad momentum spectrum. The transverse phase space distributions of the pions off two Inconel targets, which are 2.5 and 3 interaction lengths, are shown in the upper plots of Figure 4.1. The histogram of longitudinal positions where the pions are emitted from the target is shown in the lower plot of Figure 4.1. Most of the pions are emitted from the downstream face of the target rather than from its sides. Horn designs and the effectiveness of their collection are described in detail in Ref. [38-40].

As discussed in Chapter 3, the goal of designing the pion beamline is to transport as many pions as possible that can decay to muons within both $\Omega_{\epsilon}$ and $\Omega_{P}$. Therefore, the nuSTORM horn is required to focus the pions into a certain phase space, rather than to parallel trajectories. The nuSTORM horn design in the project proposal used the NuMI horn configuration, which was designed for the latter focusing purpose. Although recent studies have shown that a two-horn or even three-horn system can 

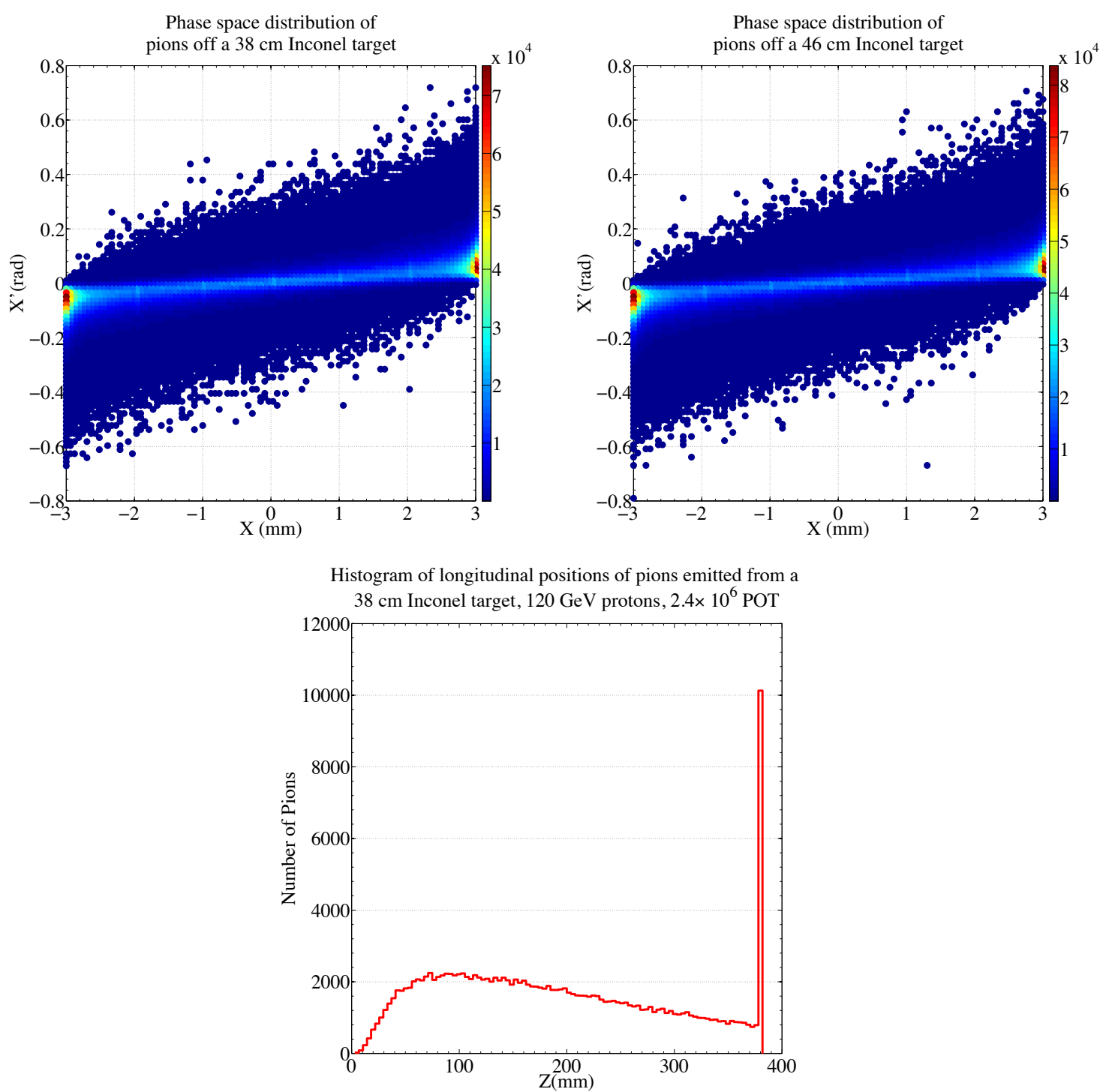

Figure 4.1: Upper: The transverse phase space histogram of the pions produced by bombarding a $38 \mathrm{~cm}$ (left) and a $46 \mathrm{~cm}$ (right) Inconel target with $120 \mathrm{GeV}$ protons, simulated by MARS. The data corresponds to $2.4 \times 10^{7}$ Protons On Target (POT). Lower: the histogram of longitudinal positions where the pions are emitted from a 38 cm target. 
be more effective than a single-horn configuration for conventional neutrino beams $[41,42]$, only one horn is considered in the proposal and also in the optimization study due to the different constraints in the nuSTORM design.

A straightforward optimization of the nuSTORM horn needs the complete tracking of pions collected by the horn through the pion beamline, with stochastic decay processes enabled in G4BL. The number of muons at the end of the pion beamline that are within the acceptance of both $\Omega_{\epsilon}$ and $\Omega_{P}$ is then compared for different horn configurations as the single fitness function value for the optimization. This is essentially a single-objective optimization problem, referred to as the number of muonsin-acceptance $\left(N_{\mu, \circ}\right.$ where $\circ$ is used to represent "circulating"). However, the full tracking simulation in the complete beamline with decay processes generally requires very large computing resources. Furthermore, for the pion phase space distribution after each horn, the optics of the pion beamline must be rematched in MAD-X, which generally adds another significant factor to the running time.

This chapter describes and demonstrates a numerical method that was developed to optimize the horn design to achieve more useful muons in the ring. The strategies used to convert the above primary optimization objective to two, in order to save computing time. This chapter also demonstrates the basic principles of the Genetic Algorithm (GA) and the Multi-Objective GA (MOGA), as well as the modifications to the algorithm that were made to improve the algorithm efficiency. The application of the technique discussed in this chapter is not limited to either the nuSTORM facility or muon based facilities, but can be used on other neutrino experiments that use magnetic horns as the collection devices. 


\subsection{MOGA}

It is possible to eliminate the need for a full tracking by considering the dynamical features of the pion beamline and the well-known two-body decay kinematics of pions. First, the single objective is reexamined with the following assumptions:

- With the beam optics from the first dipole to the end of the pion beamline unchanged, the acceptance of the pion beamline is independent of the initial pion phase space distribution (fitted phase space ellipse) as long as the optics can be matched by a conventional quadrupole capture channel. Furthermore, the number of muons in $\Omega_{\epsilon}\left(N_{\mu, \epsilon}\right)$ from pion decay is proportional to the number of pions in $\Omega_{\epsilon}\left(N_{\pi, \epsilon}\right)$ after the horn.

- Only the non-dispersive capture quads are varied to match the optics for pion beams collected by different horn configurations, so that the momentum acceptance of the pion beamline is the same for any horn collected pion beam.

- The muons in $\Omega_{P}$ at the end of the production straight $\left(N_{\mu, P}\right)$ can be estimated by the decay kinematics of the pions and the momentum acceptance of the pion beamline, which can be obtained from the statistics of the pion beam after the horn.

- Concluding assumption: The number of muons-in-acceptance can be maximized by simultaneously maximizing $N_{\mu, \epsilon}$ and $N_{\mu, P}$ that can both be derived from analyzing the pion beam at the end of the horn.

In conclusion, we are able to turn the single objective to two independent ones. The correlation between the two objectives is not analytically known to assign proper weighting to each of them, therefore, an appropriate multi-objective optimization algorithm is needed for this study. 
Table 4.1: The terminologies in GA

\begin{tabular}{|c|c|}
\hline terms in GA & meaning \\
\hline$i$ th gene & parameter $\alpha_{i}(i=1,2, \ldots, n)$ \\
\hline$j$ th individual & $\begin{array}{l}\text { one combination of the parameter set } \\
\boldsymbol{\alpha}_{j}=\left(\alpha_{1}, \ldots, \alpha_{n}\right)\end{array}$ \\
\hline population (number of individuals) & number of parameter sets \\
\hline dominance & $\begin{array}{l}\text { solution } \boldsymbol{\alpha}_{j} \text { dominates } \boldsymbol{\alpha}_{j^{\prime}} \text { if } \boldsymbol{\alpha}_{j} \text { is no } \\
\text { worse than } \boldsymbol{\alpha}_{j^{\prime}} \text { in any objective, AND } \\
\text { is strictly better than } \boldsymbol{\alpha}_{j^{\prime}} \text { in at least } \\
\text { one objective. }\end{array}$ \\
\hline natural selection & $\begin{array}{l}\text { selection of individuals based on their } \\
\text { fitness values in each objective }\end{array}$ \\
\hline parents & selected individuals \\
\hline offspring & $\begin{array}{l}\text { new individual constructed based on } \\
\text { two parents }\end{array}$ \\
\hline crossover and mutation & $\begin{array}{l}\text { new } \alpha_{i} \text { from two parent } \alpha_{i}^{\prime} \text { and } \alpha_{i}^{\prime \prime} \text { cal- } \\
\text { culated by a crossover operator and a } \\
\text { stochastic random walk. }\end{array}$ \\
\hline$k$ th generation & $k$ th iteration on parameter revolution \\
\hline Pareto front & $\begin{array}{l}\text { individuals that are not dominated by } \\
\text { any other individuals }\end{array}$ \\
\hline
\end{tabular}


The Multi-Objective Genetic Algorithm (MOGA) has been widely recognized as a powerful tool for similar purposes. Specifically, it has been adopted in a number of fields for its success in decision making problems for which the fitness values of multi-objectives are non-analytical and the global optimum is masked by many local optima. It is a heuristic method that mimics the natural selection of individuals with different chromosomes. The basic terminology is listed below in Table 4.1.

The algorithm works iteratively, namely generation by generation, until the entire population stops improving in terms of the fitness functions. The algorithm starts with the 0th population of $N$ random individuals $\left(\boldsymbol{\alpha}_{1}, \ldots, \boldsymbol{\alpha}_{N}, N\right.$ is a even number $)$, which are evaluated by $M$ fitness functions $f_{1}(\boldsymbol{\alpha}), \ldots, f_{M}(\boldsymbol{\alpha})$ for the first time. A dominance number $D_{j}$ that counts the number of other individuals that dominate a certain individual $\boldsymbol{\alpha}_{j}$ is used to sort the population for the first time. The $1^{\text {st }}$ generation of another $N$ offspring is constructed via the simulated binary crossover (SBX) (see Appendix A.2). The $2 N$ individuals are evaluated and sorted again by the objective fitness functions, from which the best $N$ individuals are selected to be

the parents for the $2^{\text {nd }}$ generation of offspring. This procedure is repeated until the population stops improving in all of the objectives.

\subsection{MOGA extended and modified}

In 2002, Deb et al. [43] developed a method to guarantee the diversity in the population, which ensures that the population does not fall into one local spot in either the parameter space or the objective space. In the method he invented, a parameter

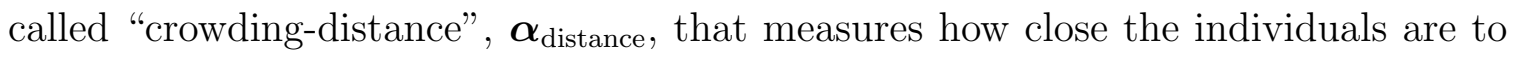
each other in the objective space (Figure 4.2).

Briefly speaking, parents that are in a crowded area have lower priority to produce 


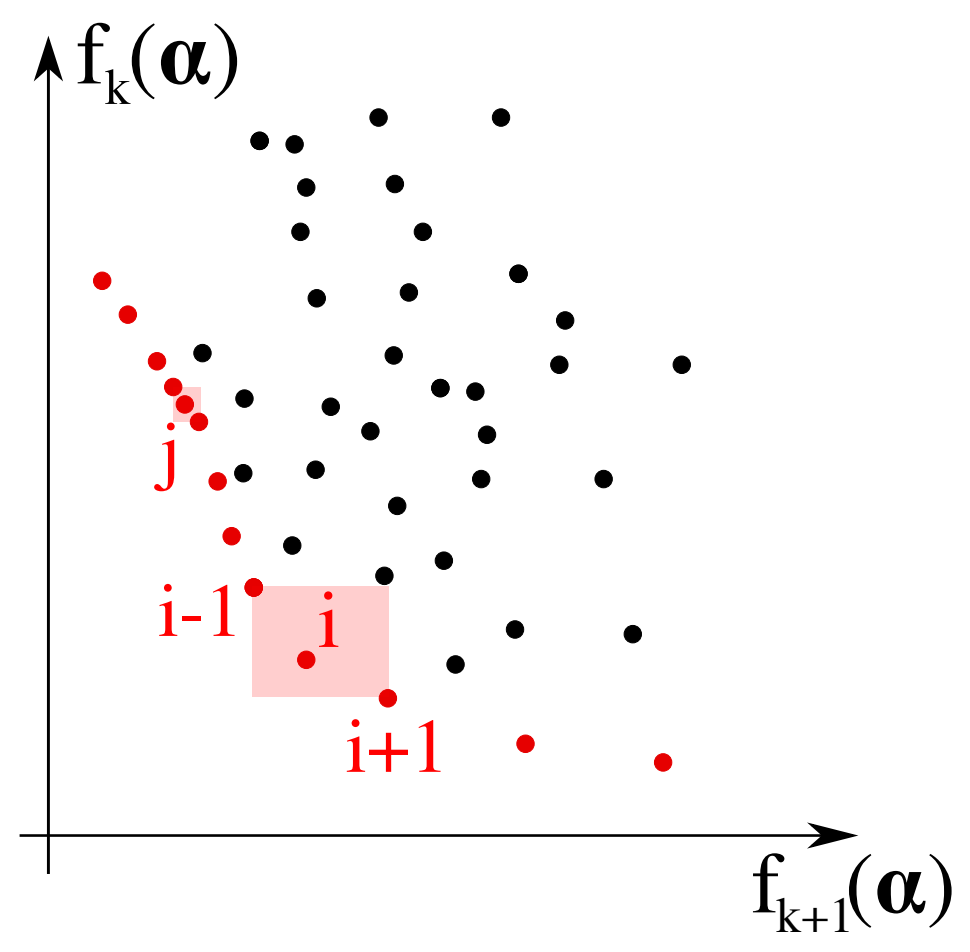

Figure 4.2: The crowding distance that estimates the density of the population surrounding one particular individual $i$ by measuring the average side length of the cuboid formed by its nearest neighbors. $f_{k}$ and $f_{k+1}$ are two objectives. In this figure, individual $i$ has larger crowding distance than $j$. 
offspring, or have a higher chance to be assigned with a mutation when producing offspring. Through this method the MOGA is able to provide a more diverse population at the end of the algorithm. It has been implemented in numerous MOGA applications.

This feature is not always needed for all decision making problems, however. Its convergence speed is usually slow because the local search activity is limited. Following the idea of "metaheuristic", which means the local multiple best candidate solutions are emphasized and improved, a modification on the usage of crowdingdistance is applied. Instead of the most crowded individuals, the most scattered and isolated individuals are more limited. It is shown that the convergence speed can be improved by up to $50 \%$, depending on the complexity of the search, with this modification.

Parallelism can be added in every offspring production stage of the algorithm, since two pairs of parents do not communicate with each other at this stage. As a matter of fact, if the objectives are evaluated independently between individuals, the entire MOGA code can be parallelized. The Message Passing Interface (MPI) was adopted in the MOGA code, which can be run on both small-scale personal computers or on large-scale computing clusters. The MOGA code is built into an optimization toolkit, called "pyOPTmpi", which uses Python to have a portable platform to connect callable SHELL programs and MOGA. The pyOPTmpi toolkit was tested on Edison of the NERSC national computing center based at Lawrence Berkeley National Laboratory (LBNL). For the horn optimization study, the time cost from tracking the pions in the horn and queuing the jobs can be greatly reduced with the MPI feature added. 


\subsection{Objective 1: Maximizing $N_{\mu, P}$}

In order to estimate the number of muons within $\Omega_{P}=3.8 \pm 10 \% \mathrm{GeV} / \mathrm{c}$ at the end of the production straight, both the momentum acceptance of the pion beamline and the pion decay kinematics are considered in the discussion below. nuSTORM is capable of delivering either $\pi^{+}$and $\mu^{+}$or $\pi^{-}$and $\mu^{-}$and perform the corresponding neutrino measurements. This section uses $\pi^{+}$and $\mu^{+}$as a representation of both species.

\subsubsection{Decay kinematics of a single pion with momentum $p_{\pi}$}

Considering a single $\pi^{+}$, the two-body decay kinematics for $\pi \rightarrow \nu_{\mu}+\mu^{+}$is readily given by

$$
\begin{aligned}
p^{2} & =\gamma^{2}\left(p_{\mu}^{\prime} \cos \theta^{\prime}+\frac{\beta E_{\mu}^{\prime}}{c}\right)^{2}+p_{\mu}^{\prime 2} \sin ^{2} \theta^{\prime} \\
& =\left(\gamma^{2}-1\right) p_{\mu}^{\prime 2} \cos ^{2} \theta^{\prime}+2 \gamma^{2} \frac{\beta E_{\mu}^{\prime}}{c} p_{\mu}^{\prime} \cos \theta^{\prime}+p_{\mu}^{\prime 2}+\gamma^{2} \frac{\beta^{2} E_{\mu}^{\prime 2}}{c^{2}}
\end{aligned}
$$

where $p, E_{\mu}$ are the momentum and energy of the $\mu^{+}$in the lab frame and $p_{\mu}^{\prime}=29.81$ $\mathrm{MeV} / \mathrm{c}, E_{\mu}^{\prime}=109.8 \mathrm{MeV}$ are the momentum and energy of the $\mu^{+}$in the rest frame of the $\pi^{+}, \gamma$ and $\beta$ are the relativistic factors of the $\pi^{+} . \theta^{\prime}$ is the decay angle in the rest frame of the $\pi^{+}$with respect to the $\pi^{+}$'s direction in the lab frame. $p$ is a function of only $\cos \theta^{\prime}$ for a certain pion momentum, $p=p\left(\cos \theta^{\prime}\right)$

From Equation (4.1), in order to get the distribution of the muon momenta $p$ in the lab frame, the distribution in $\cos \theta^{\prime}$ is required. Consider the symmetry of the pion decay in its rest frame, the distribution of muons must be uniform over the solid angle, namely $f_{\Omega^{\prime}}\left(\Omega^{\prime}\right)=f_{\cos \theta^{\prime}}\left(\cos \theta^{\prime}\right) f_{\phi^{\prime}}\left(\phi^{\prime}\right)$ is a constant. Notice here in order to avoid future confusion, the notation $f_{\mathbf{X}}(\mathrm{X})$ has been used to describe the 
probability density function of $\mathrm{X}$, where the bold subscript identifies the variable name that is in the density function. Since the variables $\cos \theta^{\prime}$ and $\phi^{\prime}$ are independent of each other, the following relation is readily obtained.

$$
\int f_{\Omega^{\prime}}\left(\Omega^{\prime}\right) d \Omega^{\prime}=\int_{-1}^{1} f_{\cos \theta^{\prime}}\left(\cos \theta^{\prime}\right) d\left(\cos \theta^{\prime}\right) \int_{0}^{2 \pi} f_{\phi^{\prime}}\left(\phi^{\prime}\right) d \phi^{\prime}=\int_{-1}^{1} f_{\cos \theta^{\prime}}\left(\cos \theta^{\prime}\right)=1
$$

which implies that

$$
f_{\cos \theta^{\prime}}\left(\cos \theta^{\prime}\right)=\frac{1}{2}
$$

The cumulative density function (CDF) is defined to be the probability that a random variable $\mathbf{x}$ will be found to have a value less than or equal to $x$. Combining both Equation (4.1) and (4.3), the CDF $F_{\boldsymbol{p}}(p)$ of the muon momentum $p$ in the lab frame is the probability that $\boldsymbol{p}\left(\cos \theta^{\prime}\right)$ has a value less than or equal to $p$, which can be calculated as follows,

$$
\begin{aligned}
& F_{\boldsymbol{p}}(p)=\operatorname{Prob}\left(\sqrt{\left(\gamma^{2}-1\right) p_{\mu}^{\prime 2} \cos ^{2} \theta^{\prime}+2 \gamma^{2} \frac{\beta E_{\mu}^{\prime}}{c} p_{\mu}^{\prime} \cos \theta^{\prime}+p_{\mu}^{\prime 2}+\gamma^{2} \frac{\beta E_{\mu}^{\prime 2}}{c^{2}}} \leq p\right) \\
& =\operatorname{Prob}\left(0 \leq\left[\left(\gamma^{2}-1\right) p_{\mu}^{\prime 2} \cos ^{2} \theta^{\prime}+2 \gamma^{2} \frac{\beta E_{\mu}^{\prime}}{c} p_{\mu}^{\prime} \cos \theta^{\prime}+p_{\mu}^{\prime 2}+\gamma^{2} \frac{\beta E_{\mu}^{\prime 2}}{c^{2}}\right] \leq p^{2}\right) \\
& =\operatorname{Prob}\left(\cos \theta^{\prime}{ }_{-} \leq \cos \theta^{\prime} \leq \cos \theta^{\prime}{ }_{+}\right) \\
& =\operatorname{Prob}\left(-1 \leq \cos \theta^{\prime} \leq \cos \theta^{\prime}{ }_{+}\right) \\
& =F_{\cos \theta^{\prime}}\left(\cos \theta_{+}^{\prime}\right)-F_{\cos \theta^{\prime}}(-1)
\end{aligned}
$$

where $\cos \theta^{\prime}$ - and $\cos \theta^{\prime}+$ are the two roots of the equation

$$
p^{2}=\left(\gamma^{2}-1\right) p_{\mu}^{\prime 2} \cos ^{2} \theta^{\prime}+2 \gamma^{2} \frac{\beta E_{\mu}^{\prime}}{c} p_{\mu}^{\prime} \cos \theta^{\prime}+p_{\mu}^{\prime 2}+\gamma^{2} \frac{\beta^{2} E_{\mu}^{\prime 2}}{c^{2}}
$$

The probability density function is the derivative of the cumulative density function, namely,

$$
f_{p}(p)=f_{\cos \theta^{\prime}}\left(\cos \theta_{+}^{\prime}\right) \frac{d \cos \theta_{+}^{\prime}}{d p}
$$


When $\gamma \gg 1$, the solution of Equation (4.5) can be written as

$$
\begin{aligned}
\cos \theta_{+}^{\prime} & \approx\left[-\frac{2 \gamma^{2} E_{\mu}^{\prime}}{c} p_{\mu}^{\prime}+\sqrt{\frac{4 \gamma^{4} E_{\mu}^{\prime 2}}{c^{2}} p_{\mu}^{\prime 2}-4 \gamma^{2} p_{\mu}^{\prime 2}\left({p^{\prime}}_{\mu}^{2}+\gamma^{2} \frac{E_{\mu}^{\prime 2}}{c^{2}}-p^{2}\right)}\right] / 2 \gamma^{2} p_{\mu}^{\prime 2} \\
& =\left[-\frac{2 \gamma^{2} E_{\mu}^{\prime}}{c} p_{\mu}^{\prime}+\sqrt{4 \gamma^{2}{p_{\mu}^{\prime}}^{2} p^{2}-4 \gamma^{2}{p_{\mu}^{\prime}}^{2}}\right] / 2 \gamma^{2}{p_{\mu}^{\prime}}^{2} \\
& \approx\left[-\frac{2 \gamma^{2} E_{\mu}^{\prime}}{c} p_{\mu}^{\prime}+2 \gamma p_{\mu}^{\prime} p\right] / 2 \gamma^{2} p_{\mu}^{\prime 2}
\end{aligned}
$$

where $p \gg p^{\prime}{ }_{\mu}$ has been applied. This suggests that the Equation (4.6) can be rewritten as

$$
f_{\boldsymbol{p}}(p)=\frac{1}{2} \cdot \frac{1}{\gamma p_{\mu}^{\prime}} \quad p_{\mu, \min }<p<p_{\mu, \max }
$$

where

$$
\begin{aligned}
& p_{\mu, \text { max }}=\sqrt{\gamma^{2}\left(p_{\mu}^{\prime}+\frac{\beta E_{\mu}^{\prime}}{c}\right)^{2}}=\gamma\left(p_{\mu}^{\prime}+\frac{\beta E_{\mu}^{\prime}}{c}\right) \approx 0.14 \gamma(\mathrm{GeV} / \mathrm{c}) \\
& p_{\mu, \text { min }}=\sqrt{\gamma^{2}\left(p_{\mu}^{\prime} \times(-1)+\frac{\beta E_{\mu}^{\prime}}{c}\right)^{2}}=\gamma\left(-p_{\mu}^{\prime}+\frac{\beta E_{\mu}^{\prime}}{c}\right) \approx 0.08 \gamma(\mathrm{GeV} / \mathrm{c})
\end{aligned}
$$

The above result implies that when the relativity factor $\gamma$ is much greater than 1 , the momentum distribution of $\mu^{+}$from $\pi^{+} \rightarrow \nu_{\mu}+\mu^{+}$decay within $[80 \gamma \mathrm{MeV} / \mathrm{c}, 140 \gamma \mathrm{MeV} / \mathrm{c}]$ is uniform.

\subsubsection{Decay kinematics of a pion beam with a linear momentum distri- bution}

Generally, the pions produced by bombarding the target with a high energy proton beam have a momentum distribution that is a decreasing linear function of the pion momentum (see Figure 3.11 for an example). 
Considering a $\pi^{+}$beam with $N_{0}$ pions in the momentum range of $\left[(1-m) P_{0},(1+\right.$ $m) P_{0}$ ] $(0<m<1)$ within which the above probability density function of the beam momentum can be expressed as $f_{p_{\pi}}\left(p_{\pi}\right)=a p_{\pi}+b$, where $a$ and $b$ are negative and positive numbers, respectively. It has been demonstrated by a number of runs of the target and horn simulations that the pions at the downstream face of the horn have a such linear distribution. An example is shown in Figure 4.3. The number of $\pi^{+}$in the infinitesimal momentum range $\left[p_{\pi}, p_{\pi}+d p_{\pi}\right]$ is therefore $N_{0}\left(a p_{\pi}+b\right) d p_{\pi}$.

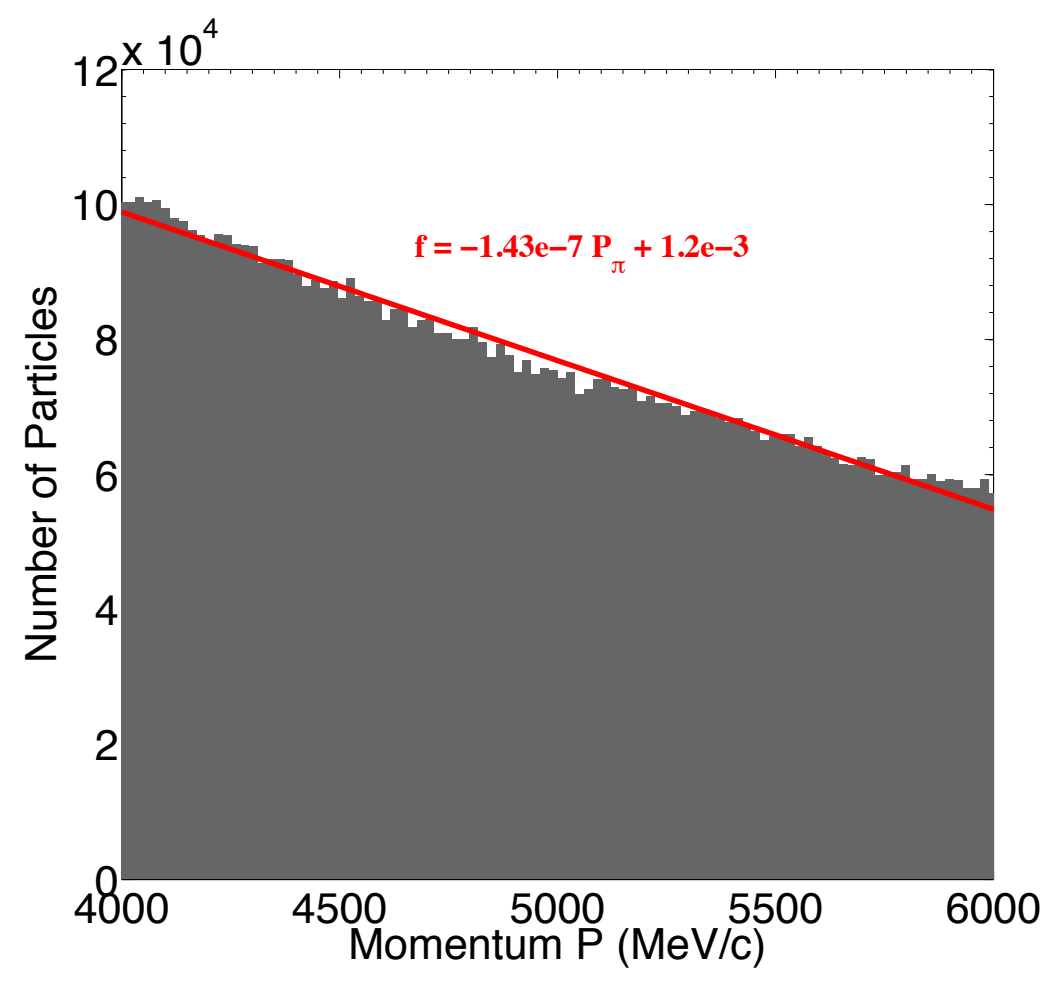

Figure 4.3: The momentum distribution of pions at the downstream face of an example horn, simulated by MARS15. The fitted probability density function and the fitting are shown in red.

Using Equation (4.8), the probability density function of the muon momentum 
distribution from pion decay of this beam can be written as the integration over the $\pi^{+}$momentum $p_{\pi}$ of the product of two density functions,

$$
\begin{aligned}
f_{\boldsymbol{p}_{\boldsymbol{\mu}}}(p) & =\int_{p_{\text {lower }}}^{p_{\text {upper }}}\left(a p_{\pi}+b\right) \cdot \frac{1}{2 \gamma p_{\mu}^{\prime}} d p_{\pi} \\
& =\int_{p_{\text {lower }}}^{p_{\text {upper }}}\left(a p_{\pi}+b\right) \cdot \frac{1}{\frac{2 p_{\pi} p_{\mu}^{\prime}}{m_{\pi}^{\prime} c}} d p_{\pi}
\end{aligned}
$$

where $\beta=1$ is used with the assumption that $\gamma \gg 1$. $p_{\text {lower }}$ and $p_{\text {upper }}$ stand for the lower and upper limits of the momentum of the pion that can decay to a muon with momentum $p$.

Recalling the maximum and minimum momentum of a muon from the decay of a pion with a relativistic factor $\gamma$ is given by Equations (4.9) and (4.10), the maximum and minimum momentum of a pion that can decay to a muon with momentum $p$ is given by

$$
\begin{aligned}
\gamma_{\max }=\frac{p_{\pi, \max }}{m_{\pi}^{\prime} c} & =\frac{p}{80}, \quad \gamma_{\min }=\frac{p_{\pi, \min }}{m_{\pi}^{\prime} c}=\frac{p}{140} \\
p_{\pi, \max } & \approx \frac{7}{4} p, \quad p_{\pi, \min } \approx p
\end{aligned}
$$

Consequently, the integration limits of Equation (4.11) are

- $p_{\text {lower }}=(1-m) P_{0}, p_{\text {upper }}=p_{\pi, \max }$,

$$
\text { if } p_{\pi, \min } \leq(1-m) P_{0} \text { and } p_{\pi, \max } \leq(1+m) P_{0}
$$

- $p_{\text {lower }}=p_{\pi, \min }, p_{\text {upper }}=(1+m) P_{0}$,

$$
\text { if } p_{\pi, \min } \geq(1-m) P_{0} \text { and } p_{\pi, \max } \geq(1+m) P_{0}
$$

- $p_{\text {lower }}=p_{\pi, \min }, p_{\text {upper }}=p_{\pi, \max }$

$$
\text { if } p_{\pi, \min } \geq(1-m) P_{0} \text { and } p_{\pi, \max } \leq(1+m) P_{0}
$$

- $p_{\text {lower }}=(1-m) P_{0}, p_{\text {upper }}=(1+m) P_{0}$,

$$
\text { if } p_{\pi, \min } \leq(1-m) P_{0} \text { and } p_{\pi, \max } \geq(1+m) P_{0}
$$


- $f_{p}(p)$,

otherwise.

Furthermore, considering the fact that most of the particles that have a momentum greater than $1.271 P_{0}$ or less than $0.729 P_{0}$ will not be accepted by the beamline with the design momentum equal to $P_{0}$, the possibility that $p_{\pi, \min } \geq(1-m) P_{0}$ and $p_{\pi, \max } \leq$ $(1+m) P_{0}$ can be eliminated by assuming $m \leq 0.271$. In other words, a beam with momentum spread $\delta P / P_{0} \leq 0.271$ is considered. As a result, $f_{p_{\mu}}(p)$ can be expressed in a piecewise function,

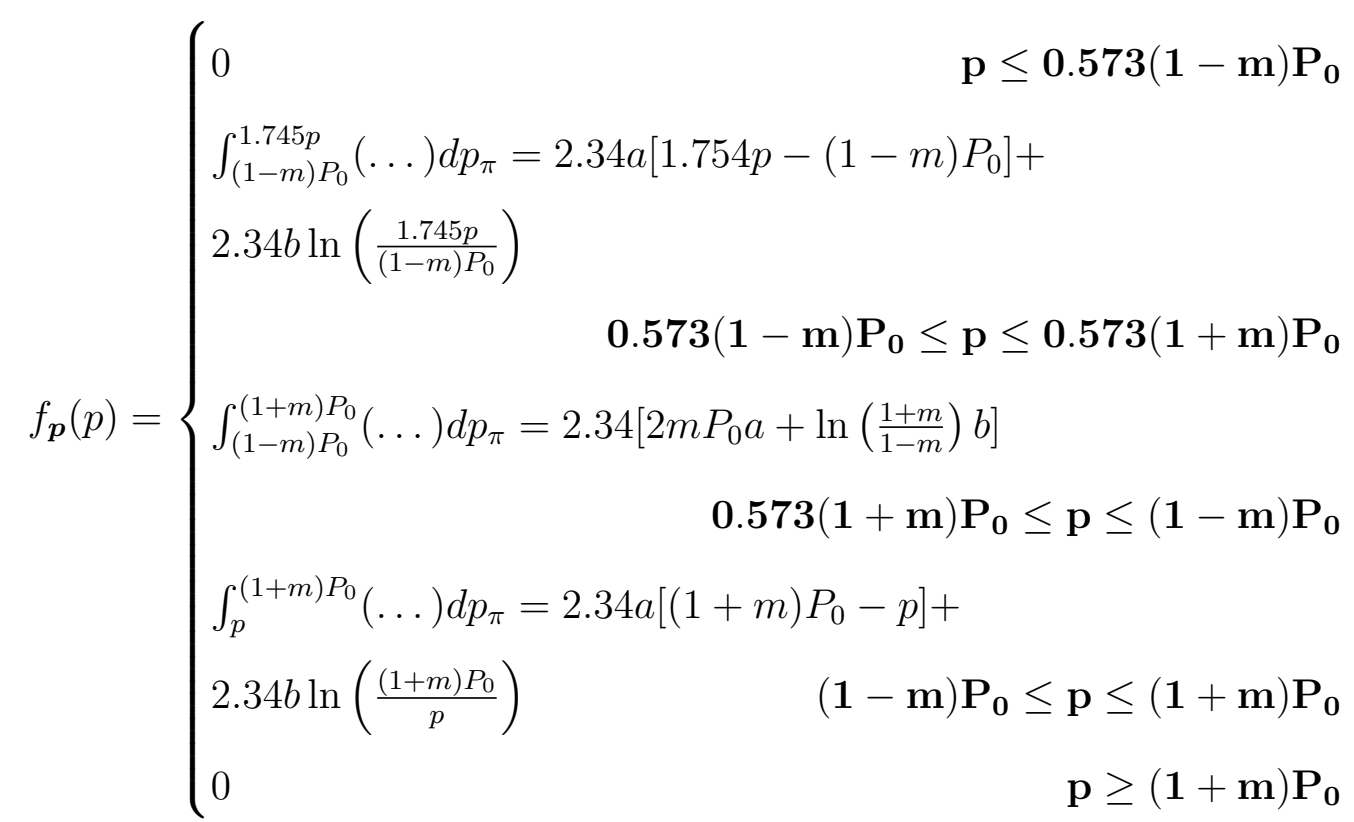

Notice that the expression for $\mathbf{0 . 5 7 3}(\mathbf{1}+\mathbf{m}) \mathbf{P}_{\mathbf{0}} \leq \mathbf{p} \leq(\mathbf{1}-\mathbf{m}) \mathbf{P}_{\mathbf{0}}$ does not explicitly depend on $p$, and the corresponding value is the global maximum of the piecewise function $f_{\boldsymbol{p}}(p)$ for a given $m$ and $P_{0}$. The probability density function $f_{\boldsymbol{p}}(p)$ is compared with the MC data from a G4BL simulation and with the analytical values from Equation (4.13) in Figure 4.4. A sample $\pi^{+}$beam with $m=0.1$ and $P_{0}=5$ $\mathrm{GeV} / \mathrm{c}$ is forced to decay instantaneously in the simulation so that the muons could 
be recorded.

Accordingly, the maximum number of muons within $\Omega_{P}$ that can be obtained from such a pion beam is found when the $3.8 \pm 10 \% \mathrm{GeV} / \mathrm{c}$ region is completely within the pion momentum range of $\left[0.573(1+m) P_{0},(1-m) P_{0}\right]$. The maximum number of muons from these $N_{0}$ pions is thus

$$
N_{\mu, \max }=2.34\left[2 m P_{0} a+\ln \left(\frac{1+m}{1-m}\right) b\right] \times 760 \times N_{0}
$$

Since $a$ is negative, $N_{\mu, \max }$ is thus inversely proportional to $P_{0}$. The choice of $P_{0, \pi}=5$ $\mathrm{GeV} / \mathrm{c}$ was chosen to be a balance between more $\mu^{+}$in the ring acceptance, which is shown in Equation (4.14), and lower dispersion needed at the OCS for combining the pion and muon orbits. The above expression then becomes

$$
N_{\mu, \max }=1.78 \times 10^{3} N_{0}\left[1 \times 10^{4} m a+\ln \left(\frac{1+m}{1-m}\right) b\right]
$$

when the following condition is satisfied.

$$
\begin{gathered}
0.573 \times 5(1+m) \leq 3.8-0.38, \quad 5(1-m) \geq 3.8+0.38 \\
m \in[0,0.164]
\end{gathered}
$$

Equation (4.16) suggests that if the $N_{0}$ pions have a momentum spread that is less than $16.4 \%$ centered at $5 \mathrm{GeV} / \mathrm{c}$, the muons within $3.8 \pm 10 \% \mathrm{GeV} / \mathrm{c}$ is flat and maximized. This can be shown by plotting $f_{\boldsymbol{p}}(p)$ from pion beams for $m=0.164$ (red curve) and $m=0.2$ (black curve) but with the same $N_{0}$ and $P_{0, \pi}$.

\subsubsection{Estimating the the number of $\mu^{+}$in $\Omega_{P}$ at the end of the production straight}

In order to get the final number of $\mu^{+}$at the end of the production straight, the momentum acceptance of the pion beamline must be taken into account. Using the 


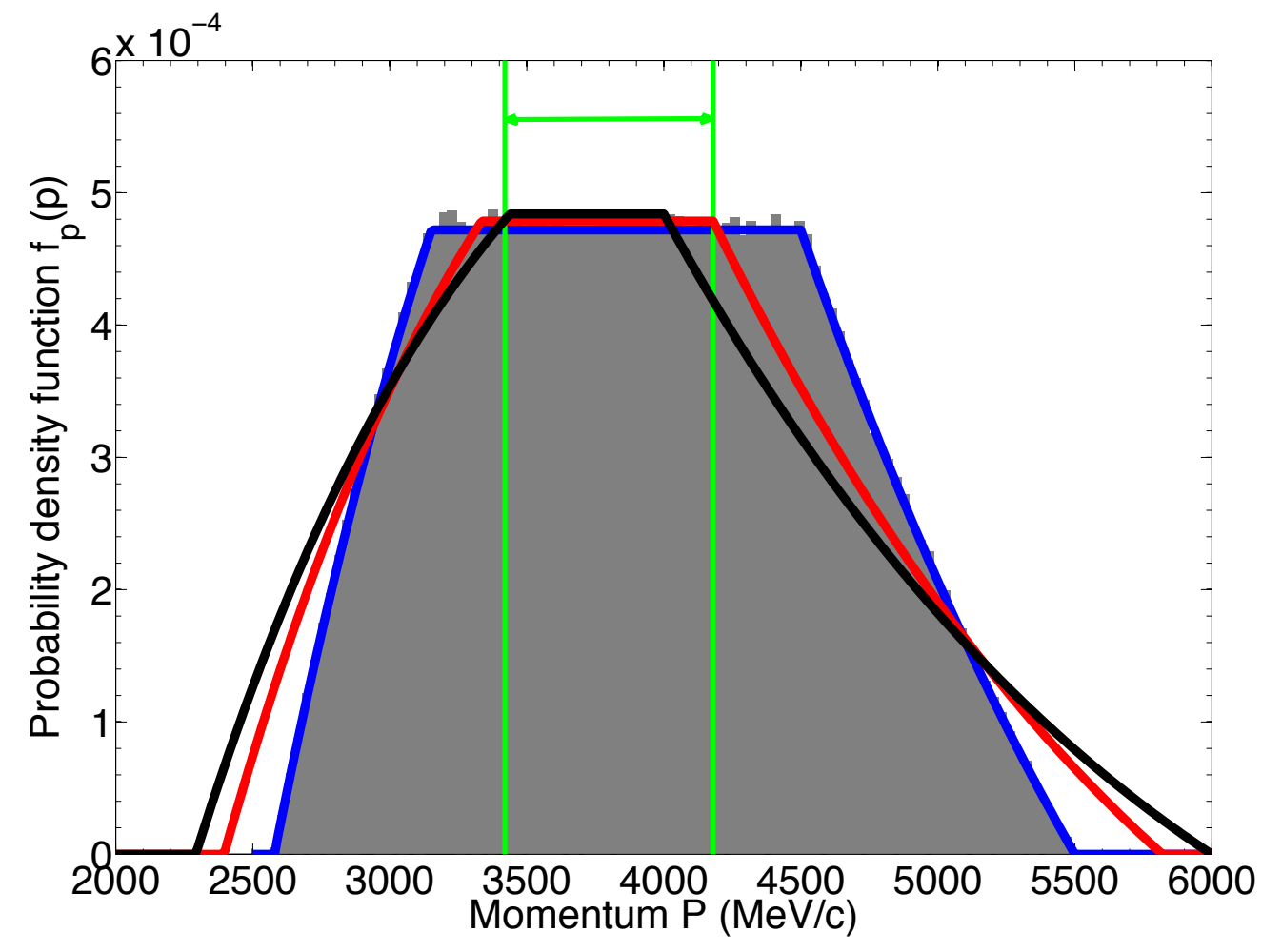

Figure 4.4: Comparison of the density function $f_{\mathbf{p}}(p)$ obtained from the analytical formula in Equation (4.13) (blue solid line) and sampling the MC decay data in G4BL (gray bar plot), using a pion beam with $m=0.1, P_{0}=5 \mathrm{GeV} / \mathrm{c}$. The red and black curves are obtained by Equation(4.13) for pion beams with $m=0.164$ and $m=0.2$, respectively. $N_{0}$ and $P_{0}$ are the same for all curves. The $\Omega_{P}$ range is marked in green. 
G4BL tracking discussed in Section 3.5, the momentum acceptance can be evaluated in the following way. The tracking is done for theoretically generated pion beams with different $m$, but the same transverse phase space admittance $\Omega_{a d}$. The transmission efficiency is then approximated as a $4^{\text {th }}$ order polynomial function of $m$, shown in Figure 4.5. The accuracy of the approximation can be tested by tracking the MARS simulated pion beam after the nuSTORM baseline horn, which is also shown in Figure 4.5 .

The equation in Figure 4.5 can be used in estimating the momentum acceptance of pion beams with matchable Twiss parameters.

With Equation (4.15) and the function in Figure 4.5, the number of muons within $3.8 \pm 10 \% \mathrm{GeV} / \mathrm{c}$ at the end of the pion beamline can be determined by

$$
N_{\mu, P}(m)=1.78 \times 10^{3} N_{0}\left[1 \times 10^{4} m a+\ln \left(\frac{1+m}{1-m}\right) b\right] \operatorname{Prob}(m) \operatorname{Prob}\left(\Omega_{a d}\right)
$$

for $m \leq 0.164$, where $\operatorname{Prob}\left(\Omega_{a d}\right)$ is the phase space acceptance of the pion beamline for $\Omega_{a d}$, which is assumed to be a constant independent of the initial Twiss parameters. The plot of $N_{\mu, P}(m)$ from Equation (4.17) is shown in Figure 4.6, modified by Equation (4.13) for $0.164 \leq m \leq 0.2$. For comparison, $N_{\mu, P}$ from G4BL tracking of two pion beams with an Inconel target and the baseline horn and with a Graphite target are plotted in the same figure. With some simple scaling, the $N_{\mu, P}(m)$ matches well with the simulation results.

Equation (4.17) and the tracking results show that $N_{\mu, P}(n)$ reaches its maximum for $m \geq 0.18$ within stochastic fluctuation errors. The results suggest that the pions outside the $5 \pm 18 \% \mathrm{GeV} / \mathrm{c}$ range can not contribute to providing more muons within the momentum acceptance of the ring. Thus, this critical value of $m=0.18$ and $N_{\mu, P}(0.18)$ can be used as a benchmark in comparing pion beams from different horn configurations. The formula to estimate the number of muons within $3.8 \pm 10 \% \mathrm{GeV} / \mathrm{c}$ 


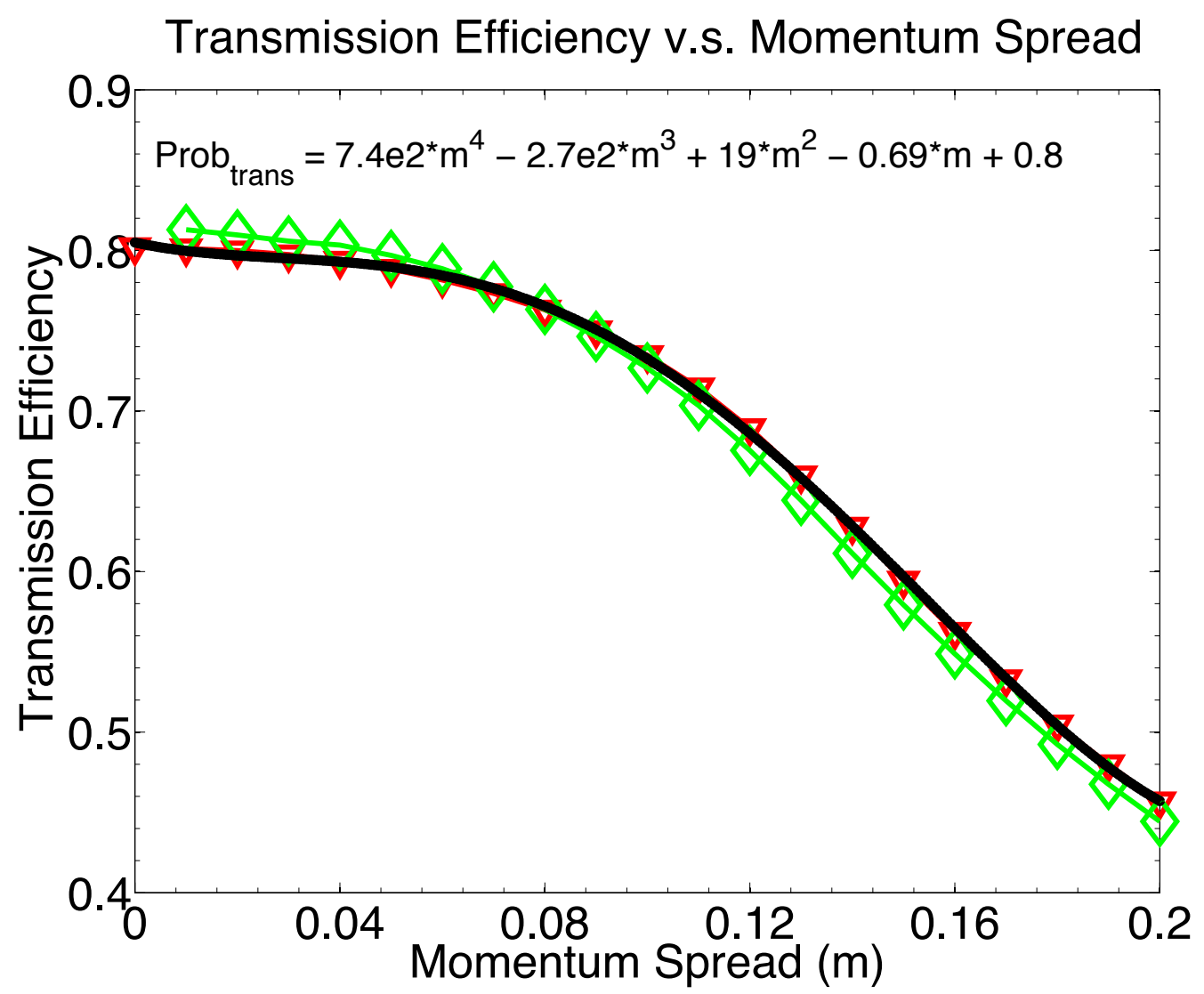

Figure 4.5: The transmission efficiency of $\pi^{+}$in the pion beamline with different momentum spreads. The tracking results from numerically generated Gaussian pion beams with different $m$ are plotted with red triangles. The function fitted to those results is plotted with the black solid line. The tracking results from the MARS simulated pion beam after the horn is plotted in green rhombuses. 


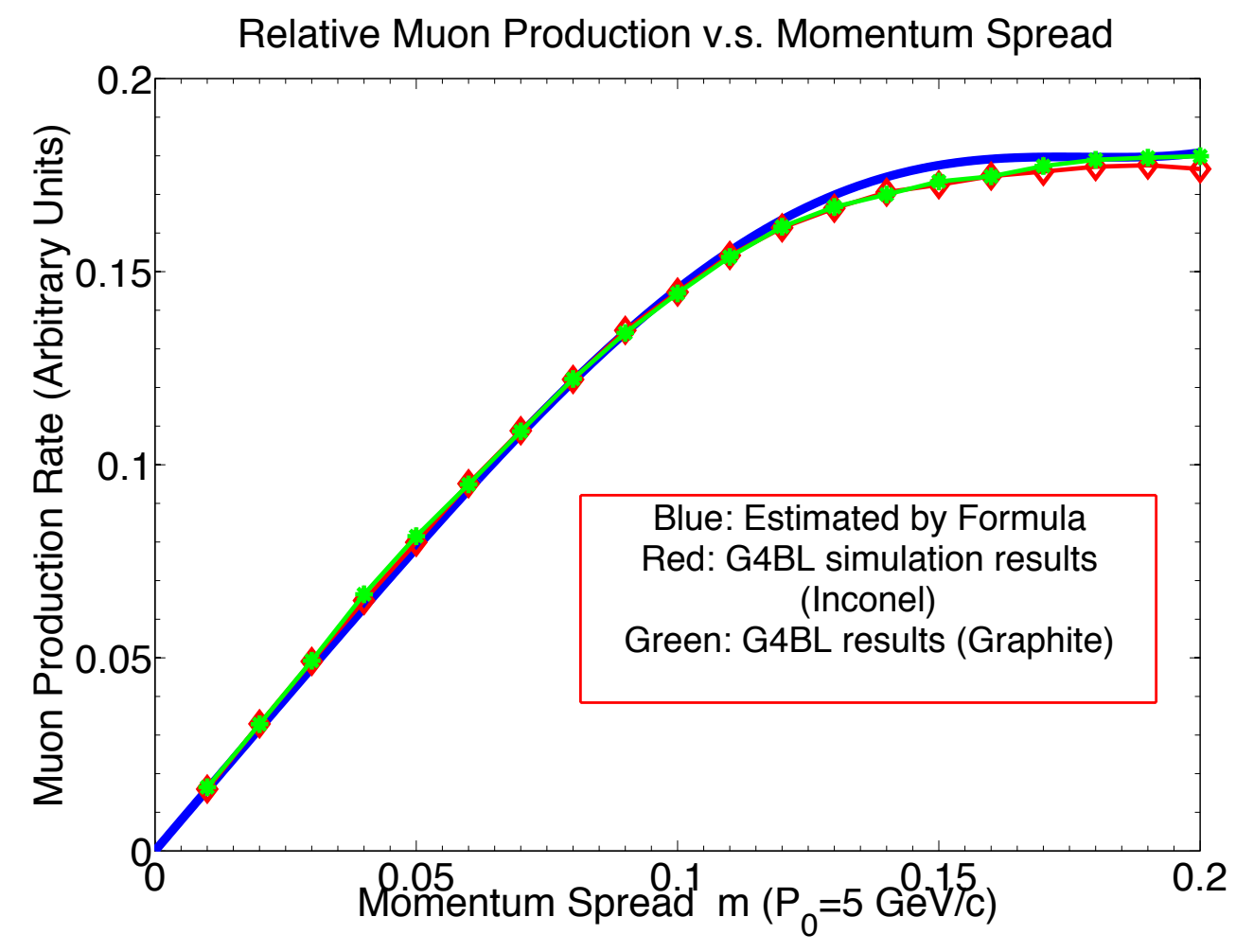

Figure 4.6: Comparison of $N_{\mu, P}(m)$ (formula) and the results from G4Beamline tracking of the pion beam from an Inconel target and the horn (red) and with the target changed to Graphite (green). The numbers are scaled to compare the normalized production rate. 
at the end of the production straight is therefore estimated to possess the following form:

$$
N_{\mu, P}(0.18) \sim 8.8 \times 10^{3} N_{0}\left[1.8 \times 10^{2} a+3.6 b\right] \operatorname{Prob}\left(\Omega_{a d}\right)
$$

where $N_{0}, a$ and $b$ in this expression are obtained at the downstream end of the horn for the momentum range of $5 \pm 18 \% \mathrm{GeV} / \mathrm{c}$. $\Omega_{a d}$ is explained in Equation 4.17. For the same target but different horns, the parameters $N_{0}, a$ and $b$ change accordingly. The first objective in MOGA for this case is to maximize $N_{\mu, P}(0.18)$. For convenience the brackets and the 0.18 are omitted in the rest of the chapter.

\subsection{Objective 2: Maximizing $N_{\pi, \epsilon}$}

To improve the transverse phase space acceptance, the $N_{0}$ pions should be focused into the $\Omega_{a c}=2000 \mu \mathrm{m}$ as much as possible, which is the second objective in this MOGA optimization. However, there is a limited range of initial phase space distributions, described by the Twiss parameters, that can be matched using conventional capture quads (pole-tip field is below $2 \mathrm{~T}$ ). This range is found by attempting to match each $(\alpha-\beta)$ combination in the MADX matching module and obtaining a list of successfully matched values. The Twiss parameters outside this range can not be matched, and therefore the acceptance is not guaranteed even though there could be more particles in the $\Omega_{a c}$. In order to save the computing time spent on rematching the optics and reconstructing the G4BL lattice, the range of feasible Twiss parameters is obtained before an optimization and used as constraints in this MOGA. The feasible range of Twiss parameters is plotted in Figure 4.7.

The IGN method developed in Section 3.4.2 is applied to obtain the Twiss parameters for different horn collected pion beams. Each combination of Twiss parameters 


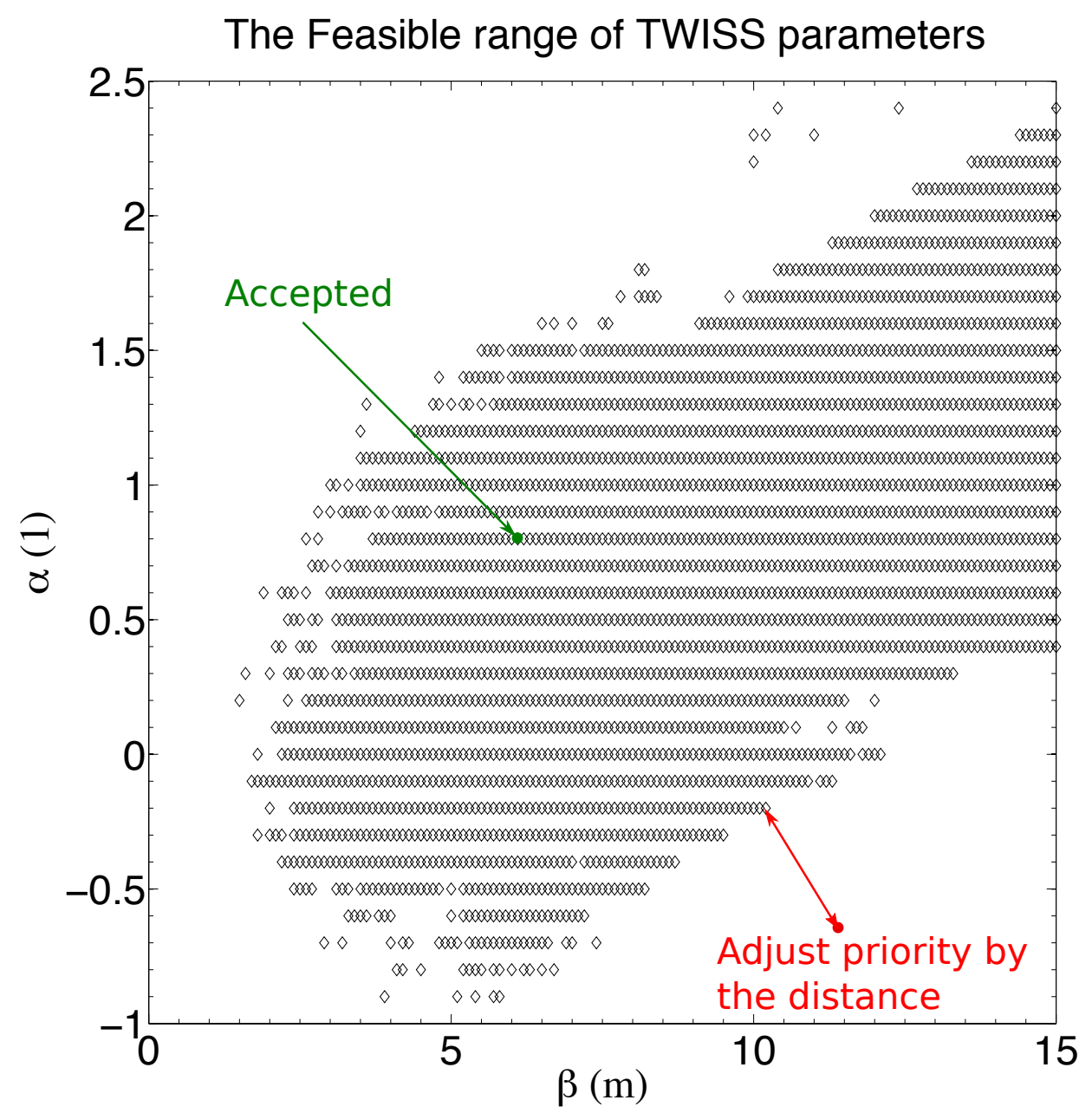

Figure 4.7: The feasible range of Twiss parameters at the downstream end of the horn that can be matched by conventional capture quads. The fitness of horns that yield Twiss parameters outside the range and their priorities in making the offspring are evaluated using the distance to the boundary of the feasible range. 
is checked by its position relative to the successfully matched range. If the parameters are outside the range, the distance to the boundary is calculated and used as a scaling parameter in the MOGA sorting process. The further that distance is, the less priority the corresponding horn gets in producing offspring for the next generation. After the Twiss parameters are found for each MOGA individual, the number of pions in $\Omega_{a c}\left(N_{\pi, \epsilon}\right)$ is assigned as the fitness value for the second objective.

\subsection{Application of pyOPTmpi}

\subsubsection{Algorithm flowchart}

Using the objectives defined above, the MOGA built into pyOPTmpi is applied. Eight of the nine genes in this MOGA search are shown in Figure 4.8 with the schematic drawing of a horn. The ninth gene is the current $I$ in the horn. Additional constraints in the MOGA arise from engineering limitations on the horn. These restrict the smallest size of the neck of the horn, the maximum length of the horn, and the maximum current in the horn. The Edison server of NERSC was used to provide 50 nodes, or 1200 cores for each generation of running. The population size of the MOGA was 100. Each generation takes approximately 30 minutes of the pyOPTmpi and G4BL running. The flowchart of the MOGA is shown in Figure 4.9, where the loop with the MPI implemented is marked with the blue circle.

\subsubsection{Optimization results}

The search for an optimal horn configuration was performed for three different Inconel targets, which are 2.5, 3 and 4 interaction lengths, or $38 \mathrm{~cm}, 46 \mathrm{~cm}, 60 \mathrm{~cm}$ long, respectively. The optimization results are shown by plotting the evolution of objective 


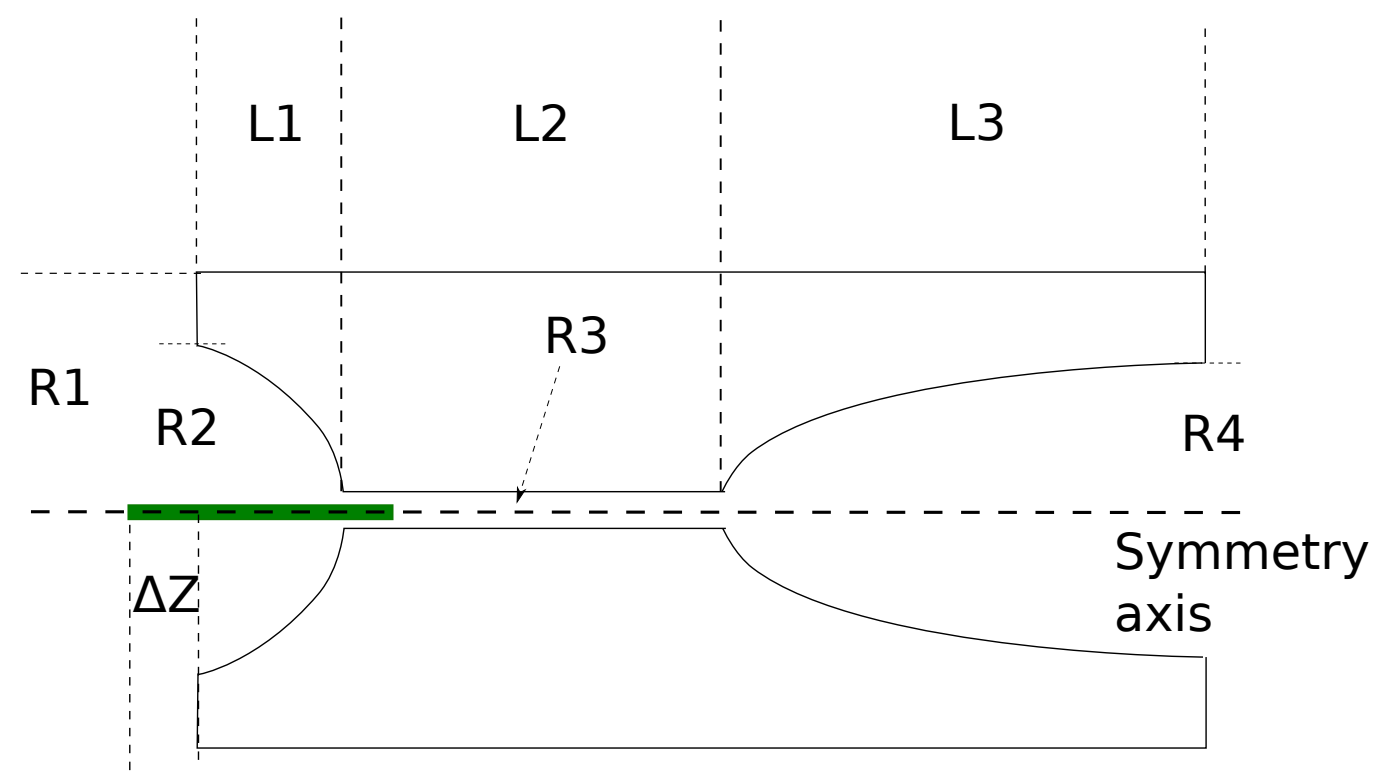

Figure 4.8: Schematic drawing of the horn showing the parameters that form the genes in the MOGA for this horn optimization study. Eight of the nine are shown by the symbols $\mathrm{L} 1, \ldots, \Delta \mathrm{Z}$, and the ninth is the horn current $I$. 


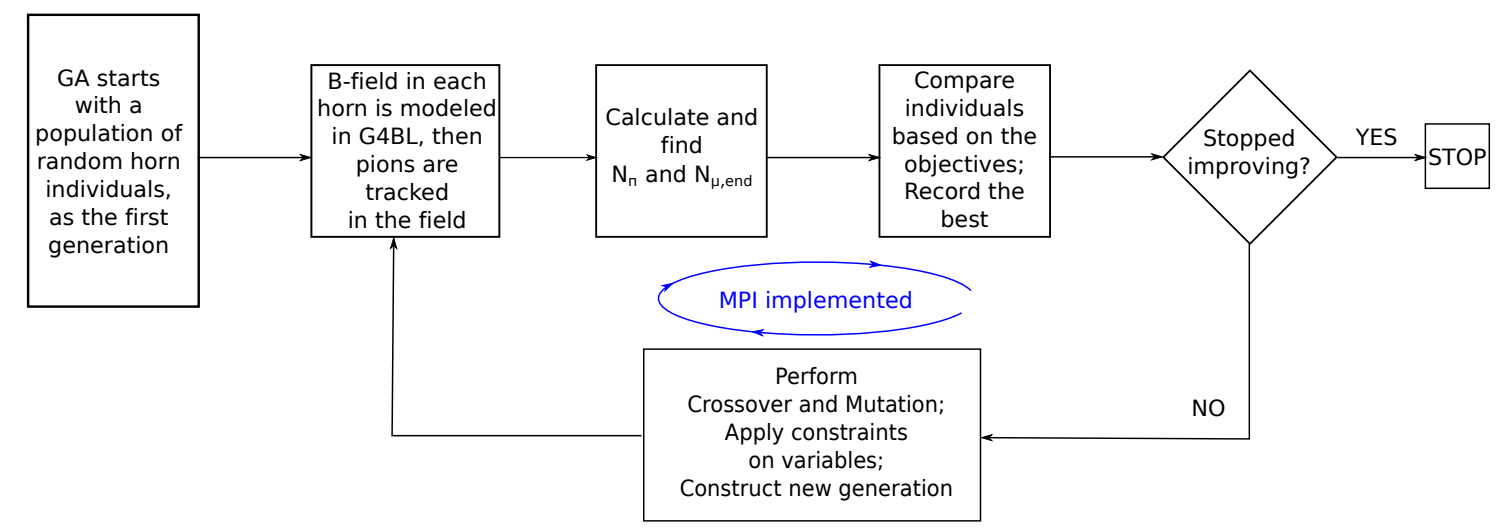

Figure 4.9: The flowchart for the MOGA applied to the horn optimization. The loop that has the MPI implemented is marked with the blue circle.

values in Figure 4.10 for the $38 \mathrm{~cm}$ and $46 \mathrm{~cm}$ targets. Increasing the target length from $46 \mathrm{~cm}$ to $60 \mathrm{~cm}$ did not show any further improvement. The pre-optimized and optimized horn configurations for a $46 \mathrm{~cm}$ Inconel target are shown in Figure 4.12. The algorithm stopped when the population ceased improving at the $81^{\text {st }}$ generation. It is found that the challenges for both horn manufacturing and operation are reduced with this new configuration. It is also expected that the optimized horn is more cost effective. The corresponding pion phase space distributions and the fitted acceptance ellipses are shown in Figure 4.11. In nuSTORM, the pion beamline is designed to transport and match the beam phase space into the ring. This is different from the simple point-to-parallel optimum of a conventional neutrino beam, such as NuMI, LBNE, etc. This can be seen in Figure 4.11 where the pions from the optimized horn have a larger angular divergence.

In order to confirm the optimization benefits, complete tracking was done in G4BL 

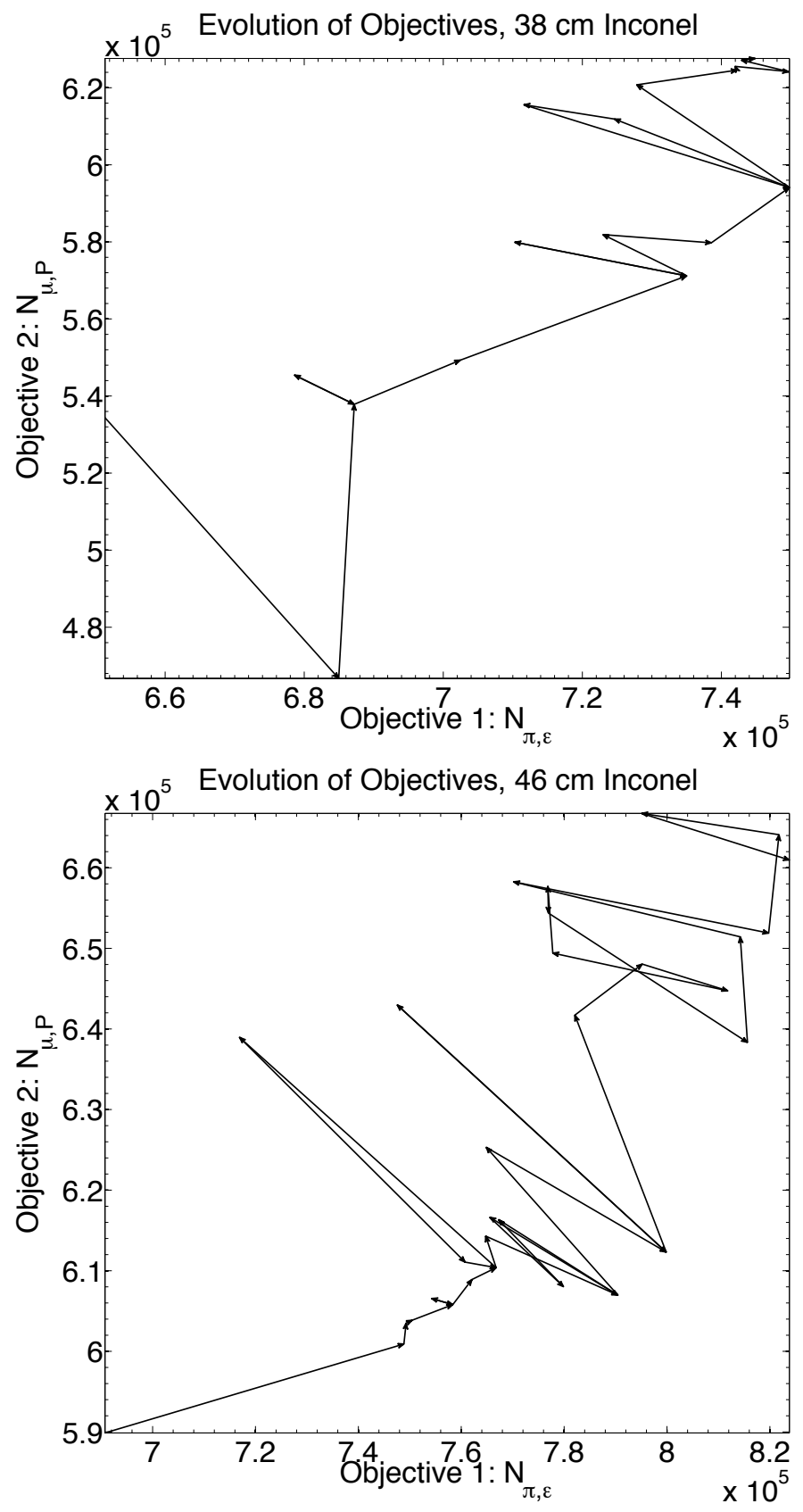

Figure 4.10: The evolution of the objective values for the two searches for optimum horn configurations with $38 \mathrm{~cm}$ (above) and $46 \mathrm{~cm}$ (below) Inconel targets. The arrows are formed when the best individual in an older generation is replaced by another in a newer generation. 

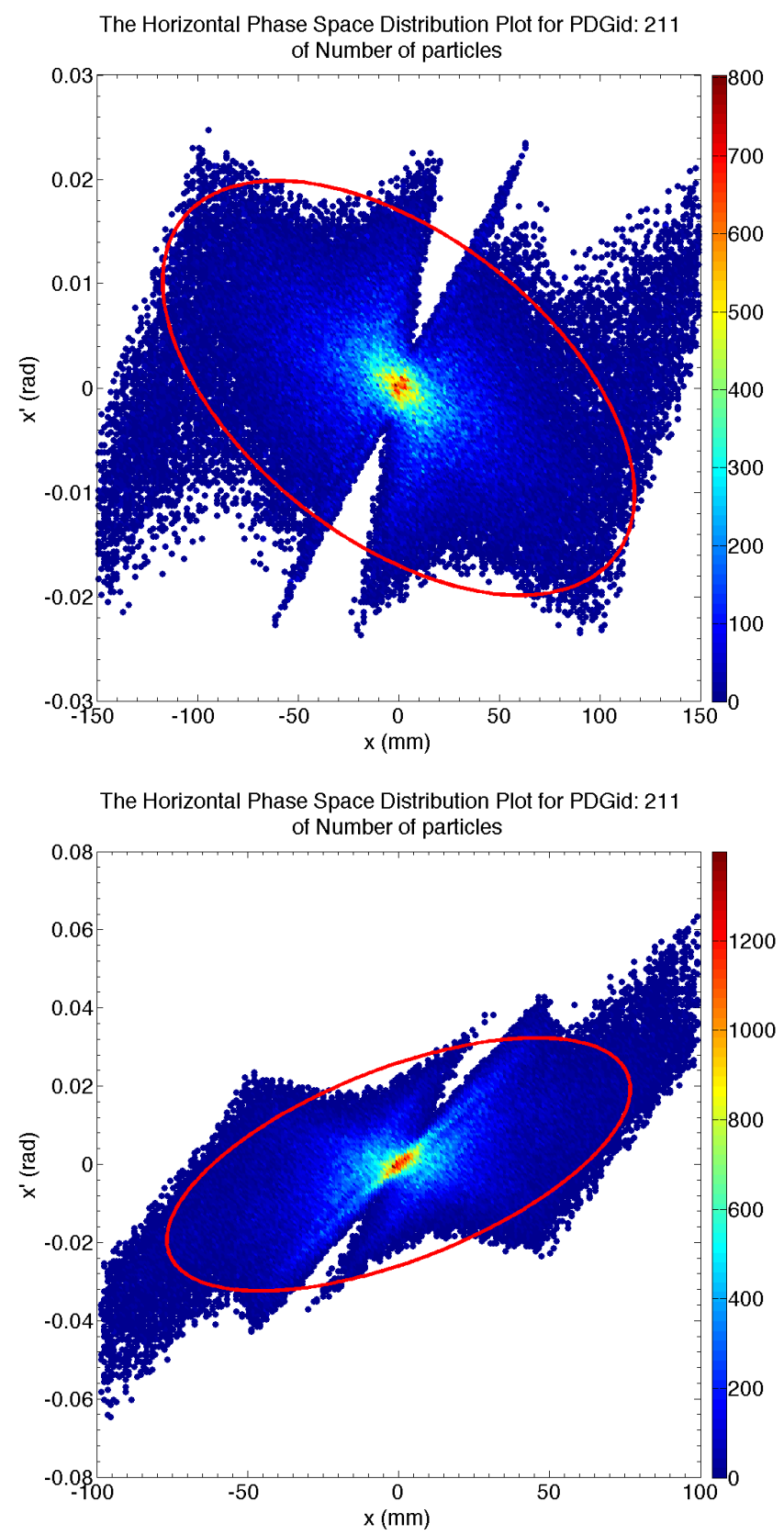

Figure 4.11: A comparison of the phase space distributions of pions focused by the pre-optimized and the optimized horn (top and bottom, respectively). The red ellipses are the fitted Gaussian acceptance ellipses. The number of pions included in the acceptance is $6.9 \times 10^{5}$ and $8.2 \times 10^{5}$ (a 1993 ncrease) from $2.4 \times 10^{6} 120 \mathrm{GeV}$ POT, respectively. For reference, $1.2 \times 10^{6}$ pions emitted from the target surface, respectively. 
with the rematched pion beamline optics to obtain the number of muons in the ring acceptance. An increase of more than $8 \%$ was found for the horn used with the 38 cm Inconel target, compared with the pre-optimized baseline model. Redesigning the horn for the $46 \mathrm{~cm}$ Inconel target yields an increase of $16 \%$ to the number of muons-in-acceptance, compared with the pre-optimized horn+target configuration. As a comparison, increasing the target length from $38 \mathrm{~cm}$ to $46 \mathrm{~cm}$ without changing the horn only provides an increase of $5 \%$. With the same decay ring acceptance, the optimization results in an increase of $16 \%$ in the neutrino flux.

\subsection{Summary}

The optimization of the nuSTORM horn and the numerical technique for the optimization was described in this chapter. The new optimization method takes advantage of the unconventional nuSTORM pion beamline and uses a new strategy for optimizing the configuration of the horn. The MOGA that is implemented in the numerical method optimizes the number of muons in the decay ring acceptance by maximizing two objective functions at the same time and reduces the requirement on the computing resources. After the optimization, two new horn shapes were found for the $38 \mathrm{~cm}$ and $46 \mathrm{~cm}$ Inconel targets, which result in an increase of $\mathbf{8 \%}$ and $\mathbf{1 6 \%}$ in the number of muons-in-acceptance and, accordingly, the $\stackrel{(-)}{\nu})$ and $\stackrel{c}{\nu}$ e flux at the detectors. 

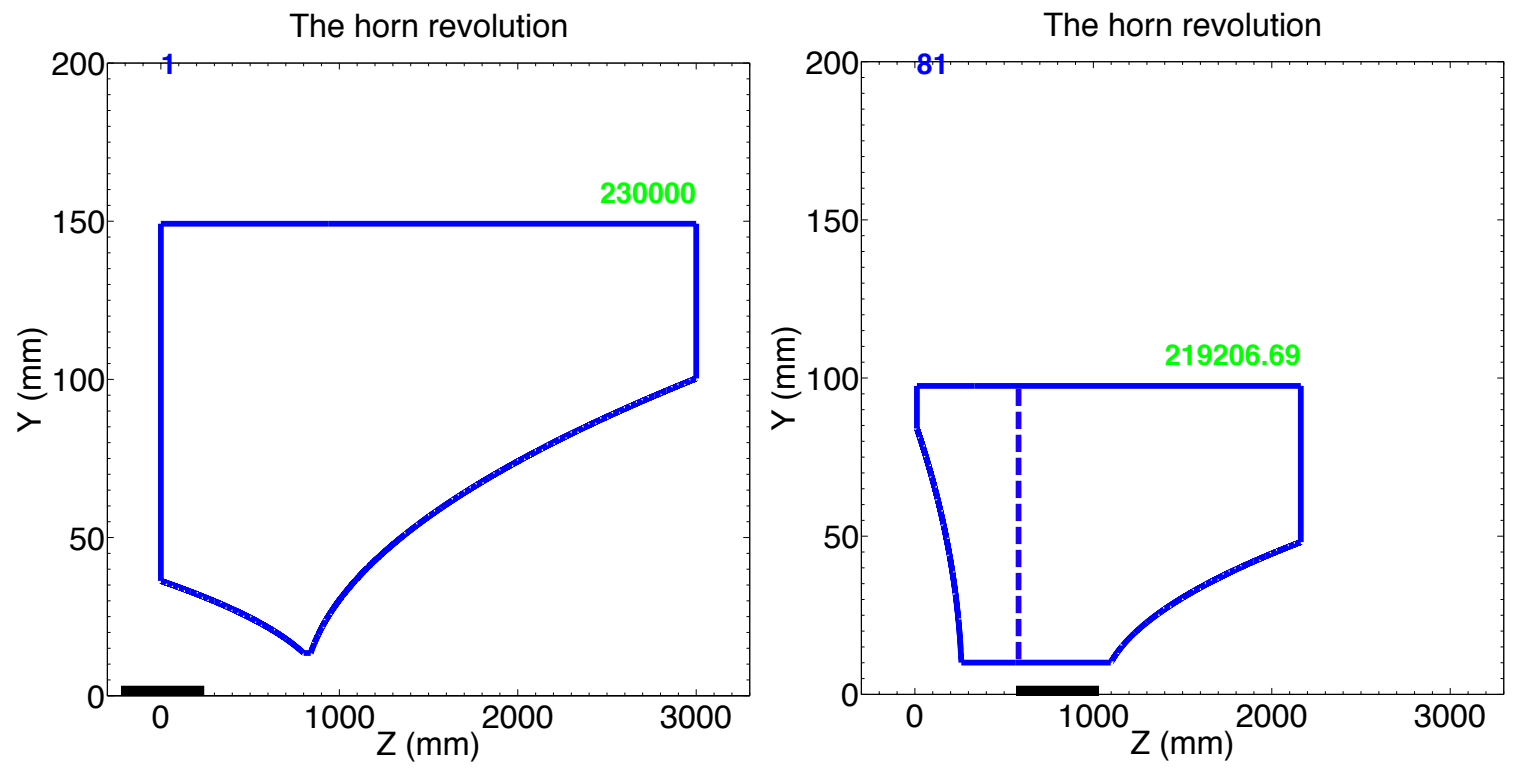

Figure 4.12: The shape of the pre-optimized horn (left) and the optimized horn (right) for a $46 \mathrm{~cm}$ Inconel target. The algorithm stopped at the $81^{\text {st }}$ generation. The effective rear boundary of the horn conductor is shown with the dashed line considering that only forward pions are useful. The optimized horn has a simpler configuration, which can reduce the maintenance and the operation costs. The optimized current is reduced from $230 \mathrm{KA}$ to $\sim 219 \mathrm{kA}$. 


\section{CHAPTER 5}

\section{nuSTORM DECAY RING DESIGN AND SIMULATION AND NEUTRINO FLUX AT nuSTORM}

In this chapter the design of the nuSTORM muon FODO decay ring and the neutrino fluxes at nuSTORM are discussed. The nuSTORM decay ring is a compact racetrack storage ring with a circumference of $\sim 480 \mathrm{~m}$ with large aperture $(\varnothing=60 \mathrm{~cm})$ magnets. The design challenges are described as follows. The dispersion created at the OCS (see Chapter 3) must be suppressed to avoid unacceptably large beam size, and this requires strong focusing and bending magnets immediately following the OCS. Moreover, in order to maximize the number of useful muon decays, strong bending dipoles are needed in the arcs to minimize the arc length. The strong focusing creates large chromaticities for the off-momentum particles, given by

$$
\begin{aligned}
& \Delta \nu_{x}=\frac{1}{4 \pi} \oint \beta_{x} \Delta K_{x} d s \\
& \Delta \nu_{z}=\frac{1}{4 \pi} \oint \beta_{x} \Delta K_{z} d s
\end{aligned}
$$

where $\Delta K_{x}$ and $\Delta K_{z}$ are gradient error terms due to the momentum offset $\delta=$ $\Delta P / P_{0, \mu}$. Including higher order terms in $\delta$ and the dispersion function, $\Delta K_{x}$ and 
$\Delta K_{z}$ can be expressed as follows [4].

$$
\begin{aligned}
& \Delta K_{x}=\left[-\frac{2}{\rho^{2}}+K+2 \frac{D}{\rho}\left(\frac{1}{\rho^{2}}-K\right)-\left(\frac{1}{\rho}\right)^{\prime} D^{\prime}+\frac{\gamma_{x}}{\beta_{x} \rho} D\right]\left(\delta-\delta^{2}+\cdots\right)+\cdots \\
& \Delta K_{z}=\left[-K+\frac{K}{\rho} D+\left(\frac{1}{\rho}\right)^{\prime} D^{\prime}+\frac{\gamma_{x}}{\beta_{x} \rho} D\right]\left(\delta-\delta^{2}+\cdots\right)+\cdots
\end{aligned}
$$

The linear chromaticity term

$$
C_{y, n a t} \approx-\frac{1}{4 \pi} \oint \beta_{y} K_{y} d s
$$

contributes to the tune shift in the first-order in $\delta$ via $\Delta \nu_{y}=C_{y, n a t} \delta$. The chromaticities may induce tune spread across the resonance $m \nu_{x}+n \nu_{y}=$ integer lines and thus need to be corrected. The first-order chromaticities can be corrected by adding sextupoles in nonzero dispersion locations following:

$$
\begin{aligned}
C_{x} & =-\frac{1}{4 \pi} \oint \beta_{x}\left[K_{x}(s)-S(s) D(s)\right] d s \\
C_{z} & =-\frac{1}{4 \pi} \oint \beta_{z}\left[K_{z}(s)-S(s) D(s)\right] d s
\end{aligned}
$$

where $S(s)$ is the sextupole strength. For larger $\delta$, the tune shifts from the second order chromaticities are stronger, which are given by $[44,45]$

$$
C_{x, z}^{(2)}=\frac{1}{2} C_{x, z}^{(1)}+\frac{1}{8 \pi} \oint[\mp K \pm S D] \frac{\partial \beta_{x, z}}{\partial \delta} d s+\frac{1}{8 \pi} \oint \pm S \beta_{x, y} D_{2} d s
$$

where $\partial \beta / \partial \delta$ and $D_{2}$ are the beta-beat from the momentum offset and the secondorder dispersion function, respectively. The higher-order dispersion terms can be written as

$$
D_{n}^{\prime \prime}+\left(\frac{1}{\rho^{2}}-K\right) D_{n}=\eta_{n}
$$


where $\eta_{n}$ are the higher-order perturbation terms of the otherwise homogeneous equation.

$$
\begin{aligned}
& \eta_{1}=\frac{1}{\rho} \\
& \eta_{2}=-\eta_{1}-\frac{1}{2} S D_{1}^{2}-\left(\frac{1}{\rho^{3}}+2 \frac{K}{\rho}\right) D_{1}^{2}+\frac{1}{2} K D_{1}^{\prime 2}+\left(\frac{1}{\rho}\right)^{\prime} D_{1} D_{1}^{\prime}+\left(\frac{2}{\rho^{2}}+K\right) D_{1}
\end{aligned}
$$

It is seen that the higher-order dispersion functions depend on the lower-order ones, and sextupoles change the $D_{2}$ term but also higher-order ones. Adding sextupoles also introduces nonlinear resonances and generates nonlinear betatron detuning following $Q_{x}=\nu_{x}+\alpha_{x x} J_{x}+\alpha_{x z} J_{z}+\cdots$ and $Q_{z}=\nu_{z}+\alpha_{x z} J_{x}+\alpha_{z z} J_{z}+\cdots$, from which the effects are strong with a large beam size. It is obvious that not all of the nonlinearities can be eliminated by adding sextupoles, thus an ideal correction is to find a balance in correcting each of these parameters, in order to minimize the higher-order effects. Individual sextupoles should be placed at locations with high dispersion $D_{1}$ and large beta function ratios $\beta_{x} / \beta_{z}$ or $\beta_{z} / \beta_{x}$.

In this chapter, two optics designs for the ring are proposed, with sextupole correction introduced to improve the acceptance. The location and strength of sextupoles or sextupolar fields in the ring are then optimized using the heuristic algorithms built into pyOPTmpi and multi-particle tracking in MAD-X. The optimization results are obtained from both the Single-Objective GA (SOGA) and an improved Simulated Annealing (SA) algorithm. The neutrino flux at nuSTORM is calculated from beam tracking and Monte Carlo simulations in G4BL, which are also presented in this chapter. 


\subsection{Decay Ring Design and Simulation}

As a general guideline for the ring design, the $D_{x}$ and large $\beta$ created at the OCS must be suppressed to a lower level in a short distance. The $D_{x}$ in the arcs should be kept small, until suppressed to zero exactly where the reference orbit is bent by $180^{\circ}$. Since muon decay in the non-production straight is not utilized for neutrino production, a small $\beta$ is preferred in order to have a smaller beam size in the non-production straight FODO cells. A larger $\beta$ is favored in the production straight because of the smaller $\sigma_{x^{\prime}}$ associated with it. The periodic $\beta$, with no other constraints or requirements on their values, such as in the production straight (see Section 3.2.2), can be adjusted to change the betatron tune of the ring lattice. Thus the ring only possesses one reflection symmetry. The circumference of the ring is approximately 480 meters, which is slightly larger than the Fermilab Booster. With arcs of approximately 60 meters, the fraction of the ring circumference that points to the detectors is $\sim 0.39$. This geometry also makes it possible to utilize the intense $\nu_{\mu}$ beam from the injected $\pi^{+}$beam and its decay into muons and muon-neutrinos and distinguish its signal from that of the $\nu_{e}$ and $\bar{\nu}_{\mu}$ beams through timing. With the lifetime of muons in the lab frame at this energy, approximately $87 \%$ of the $3.8 \mathrm{GeV} / \mathrm{c}$ muons will have decayed in 100 turns. Consequently, the number of muons that survive 100 turns in tracking without decay is used as the "benchmark" for comparing the ring designs. In this section, two lattice designs, together with their linear optics and their optimizations using sextupole corrections, are presented. 


\subsubsection{Arc design with superconducting magnets}

\section{Linear Optics and the FMA}

In order to shorten the length of the arc sections, superconducting, combined-function magnets are used to bend the beam and provide transverse focusing at the same time. The arc starts at the first dispersive element (the dipole in the OCS, see Figure 3.5) and continues to the point where the dispersion is suppressed to zero entering the nonproduction straight section. Furthermore, considering Equation (5.2), the natural chromaticity can be compensated with small $\rho$ at locations with large $K(s)$ and $\beta$, which can not be achieved in separated function dipoles. The linear optics and the design parameters are shown in Figure 5.1 and Table 5.1. After the optics is determined for the arc section, a matching section is added to match both the optics to the non-production straight and match the geometry to precisely close the ring.

Table 5.1: Parameters of the decay ring design with combined function dipole arcs

\begin{tabular}{|l|l|}
\hline Parameters & Values (units) \\
\hline \hline Central momentum $P_{0, \mu}$ & $3800(\mathrm{MeV} / \mathrm{c})$ \\
Circumference & $488.5(\mathrm{~m})$ \\
Arc length & $59.8(\mathrm{~m})$ \\
Production Straight length & $181.56(\mathrm{~m})$ \\
Non-production Straight length & $187.34(\mathrm{~m})$ \\
$\left(\nu_{x}, \nu_{y}\right)$ & $(8.12,4.63)$ \\
$\left(d \nu_{x} / d \delta, d \nu_{y} / d \delta\right)$ & $(-4.11,-6.62)$ \\
\hline
\end{tabular}

In order to find the tune footprint of the ring lattice, a Frequency Map Analysis (FMA) method, analyzed by elegant [46], is employed to gain more insight, especially on the nonlinear properties of the ring. First the tune shifts with amplitude are checked with on-momentum particles. The FMA of on-momentum particles with 


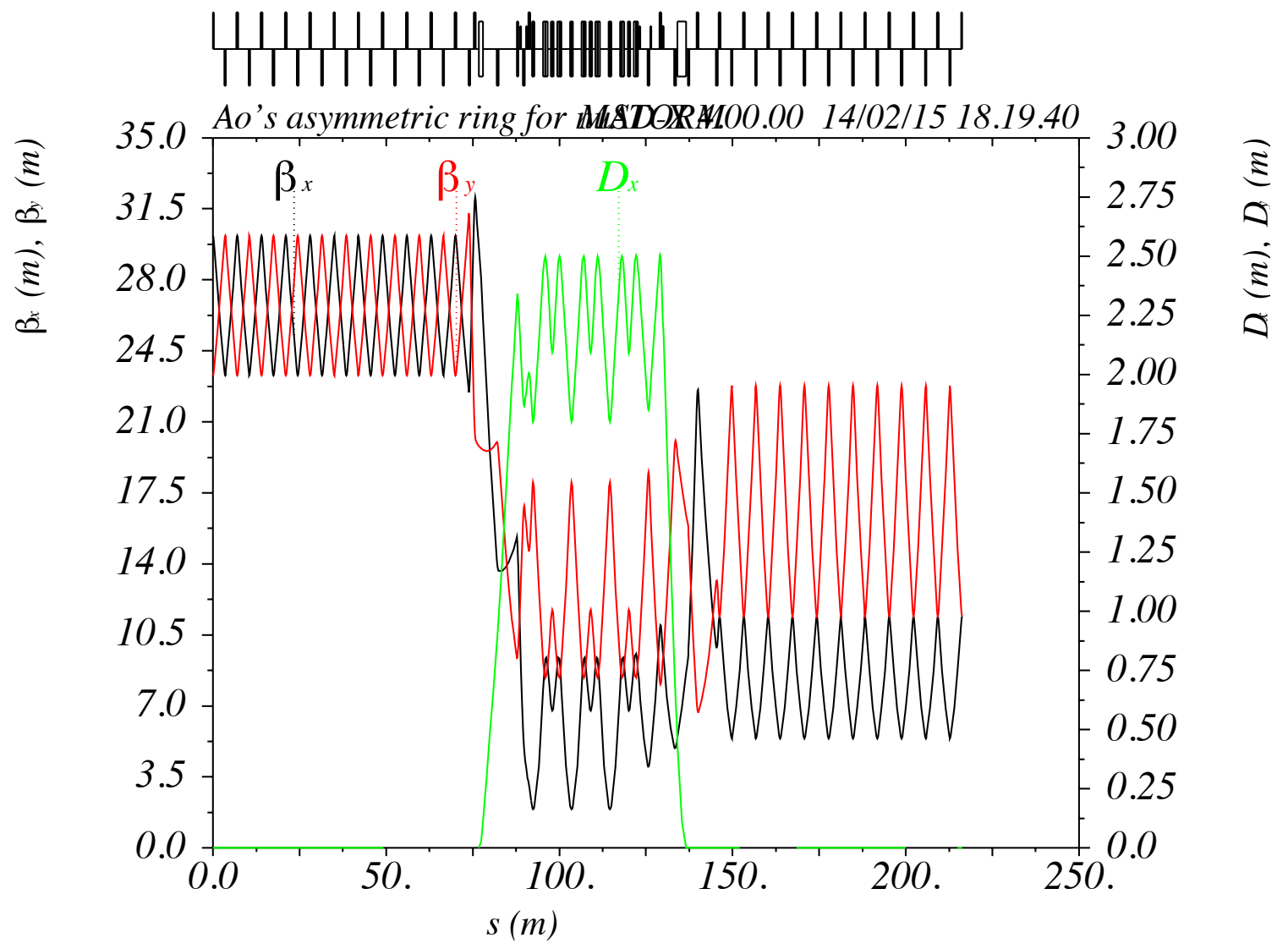

Figure 5.1: The linear optics of a nuSTORM decay ring design with superconducting combined function dipoles in the arcs. $\beta_{x}$ : black, $\beta_{y}$ : red, $D_{x}$ : green. 
proper aperture limits added in elegant is shown in the upper plots of Figure 5.2. The chromatic effects of this design are checked by doing the FMA analysis on particles with a full momentum range of $\delta \in \pm 10 \%$, which is shown by the lower plots of Figure 5.2.

The natural chromaticities are greatly reduced in this scenario, as in an ordinary FODO ring design the chromaticities are slightly larger than the betatron tunes of the ring. The tune dependence on the particle action is small for this design, however the chromatic driving terms still dilute the tune distribution in the tune plane without a clear pattern. Because of the nonlinear properties of the ring, the FMA in the frequency plane showed scattered distributions on the tune plane, with many stop bands. The acceptance is not clear from the plots, and thus needs to be obtained from symplectic tracking. The tracking was done for a muon beam with Gaussian transverse distribution, for which the transverse admittance is $2000 \mu \mathrm{m} \cdot \mathrm{rad}$, and uniform momentum distribution within $3.8 \pm 10 \% \mathrm{GeV} / \mathrm{c}$. Without the sextupole correction, the number of particles surviving 100 turns is $58 \%$.

\subsubsection{Sextupole correction optimization}

Sextupole corrections were added in the ring in order to improve the acceptance of the ring. Due to the limitations on the available space between magnets, and to keep the length of the arcs short, sextupole fields were also added to the quadrupoles in the arcs to form combined function multipoles, which are limited to have less than 4 Tesla pole-tip fields. This allows 3 combined function multipoles and three individual sextupoles, all at dispersive locations, to carry sextupole fields.

The optimization was done with both the GA and the Simulated Annealing (SA) algorithm. The basic concepts of SA are described in the Appendix A.3. It is recog- 


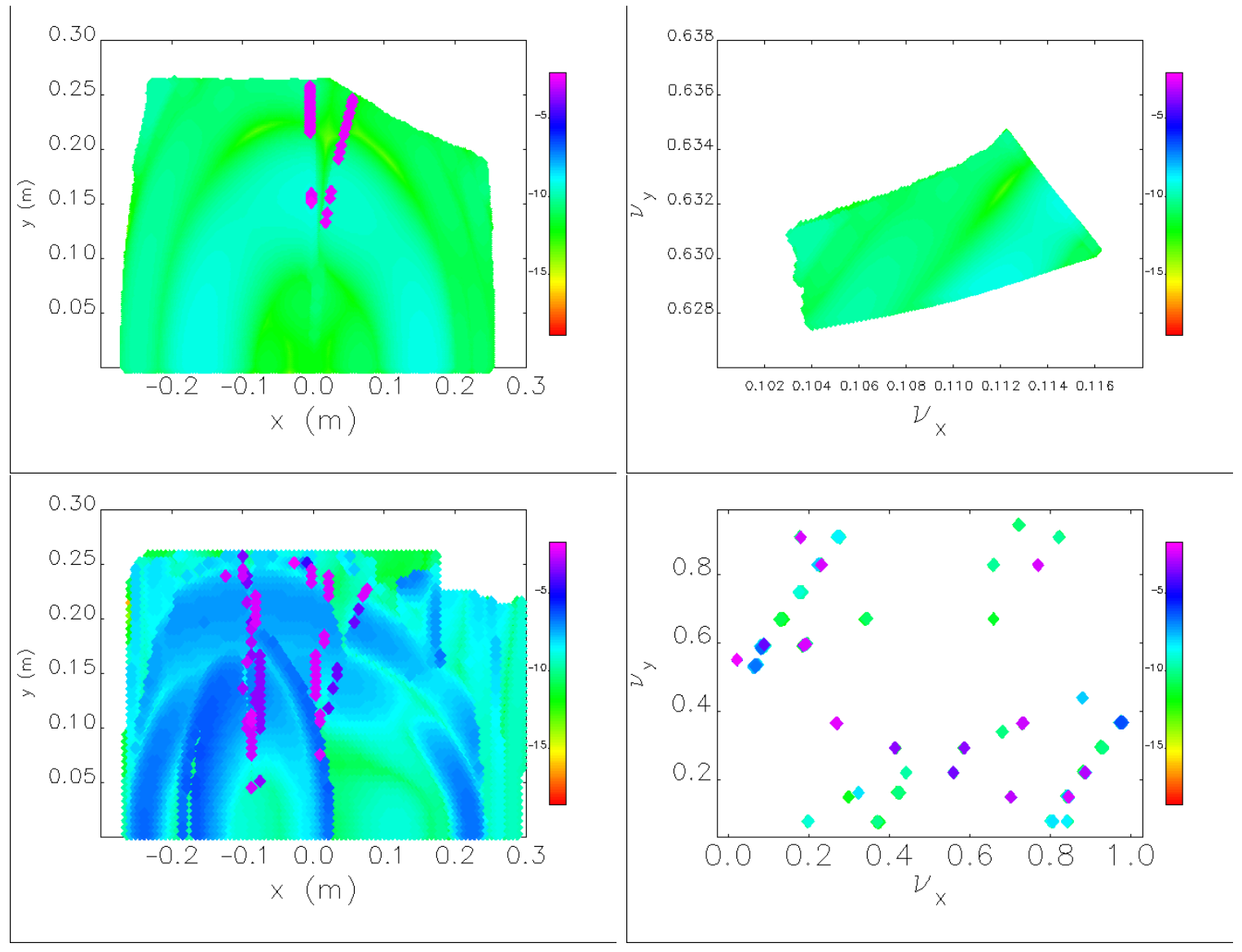

Figure 5.2: The Frequency Map Analysis (FMA) of the ring design with combined function dipoles in the arcs in the $\mathrm{X}-\mathrm{Y}$ space (left) and on the betatron tune plane (right), on-momentum particles (upper) and off-momentum particles within the full range of $\delta= \pm 0.1$ (lower) are analyzed. No sextupole corrections added. 
nized that one of the biggest disadvantages of a single-process SA algorithm is that it loses record of the good solutions at the beginning stage of the algorithm, in which the temperature $T$ that controls the selection probability, Prob $=\exp (-\Delta f / T)$, is large. It is not guaranteed that these solutions can be recovered after they are abandoned by the algorithm.

In order to improve the computing efficiency, a Message Passing Interface (MPI) was added to the standard SA routine. Moreover, the following feature was added to the standard single-process SA algorithm to mitigate the disadvantage of the singleprocess version of the algorithm and enhance the computing efficiency. In the modified SA, each MPI slave node performs an individual search for the global optimum of the problem, as in an ordinary SA. Each search done by the slave nodes is completely independent of the others. A mother node gathers the best solutions from each slave node in every step, and focuses on the neighborhood of the best solution in the entire algorithm run. This prevents information loss in the early stages of the algorithm, and increases the odds of finding a considerably better optimum in a short running time, even if some of the slave processes fail to converge to the global optimum.

In this study, the single objective of the optimization is to increase the number of the particles that may survive 100 turns. The fitness value for this objective is the percentage of the initial muons that can survive 100 turns in multi-particle tracking computed by the MAD-X PTC_TRACK [21] module. The tracking is started from the middle of the production straight shown in Figure 5.1. The initial muon beam is generated based on the covariance matrix of the beam at that point with the momentum of particles uniformly distributed within $3.8 \pm 10 \% \mathrm{GeV} / \mathrm{c}$, and the transverse phase space normally distributed within the $2 \mathrm{~mm} \cdot \mathrm{rad}$ admittance. After the optimization, the acceptance of the beam was increased to $67 \%$ from $58 \%$. 
The FMA of the post-optimization ring design are shown in Figure 5.3. The FMA results imply that the sextupole corrections are not capable of correcting all the higher order terms.

The SA algorithm was used to find the optimal sextupole distribution in this design. The survival rate of the muon beam after 100 turns is increased from $58 \%$ to $67 \%$ from the optimization. The nonlinear chromatic terms are noticeably suppressed by the algorithm (see Table 5.2). Both the GA and SA converged to the same optimum acceptance of $67 \%$. The FMA with the sextupole corrections is shown in Figure 5.3.

Table 5.2: Comparison of the principal terms corrected by the sextupole optimization

\begin{tabular}{|l|l|l|}
\hline Parameters & Before sextupole correction & After sextupole optimization \\
\hline \hline$d \nu_{x} / d \delta$ & -4.29 & -4.61 \\
$d^{2} \nu_{x} / d \delta^{2}$ & -3.62 & 0.41 \\
$d^{3} \nu_{x} / d \delta^{3}$ & -326 & 166 \\
$d \nu_{y} / d \delta$ & -7.34 & -6.68 \\
$d^{2} \nu_{y} / d \delta^{2}$ & -10.7 & -3.79 \\
$d^{3} \nu_{y} / d \delta^{3}$ & -94.1 & -78.5 \\
$D_{2}$ & 21.2 & 1.57 \\
$D_{3}$ & 958 & 831 \\
\hline
\end{tabular}

The ring acceptance was also analyzed by calculating the average number of turns that the particles can survive before they are lost by hitting the aperture limits. The histograms of the average survival turns for particles in each momentum and emittance bin are shown in Figure 5.4 and Figure 5.5. Both the pre-optimization and post-optimization lattice tracking results are plotted and compared. It is observed from Figure 5.4 that, the acceptance of on-momentum particles is not $100 \%$ because of the large transverse emittance. There are major stopbands at two $\delta$ locations, $\delta \approx-0.05$ and $\delta \approx 0.03$. When $|\delta|$ is close to 0.1 , the acceptance is further limited 


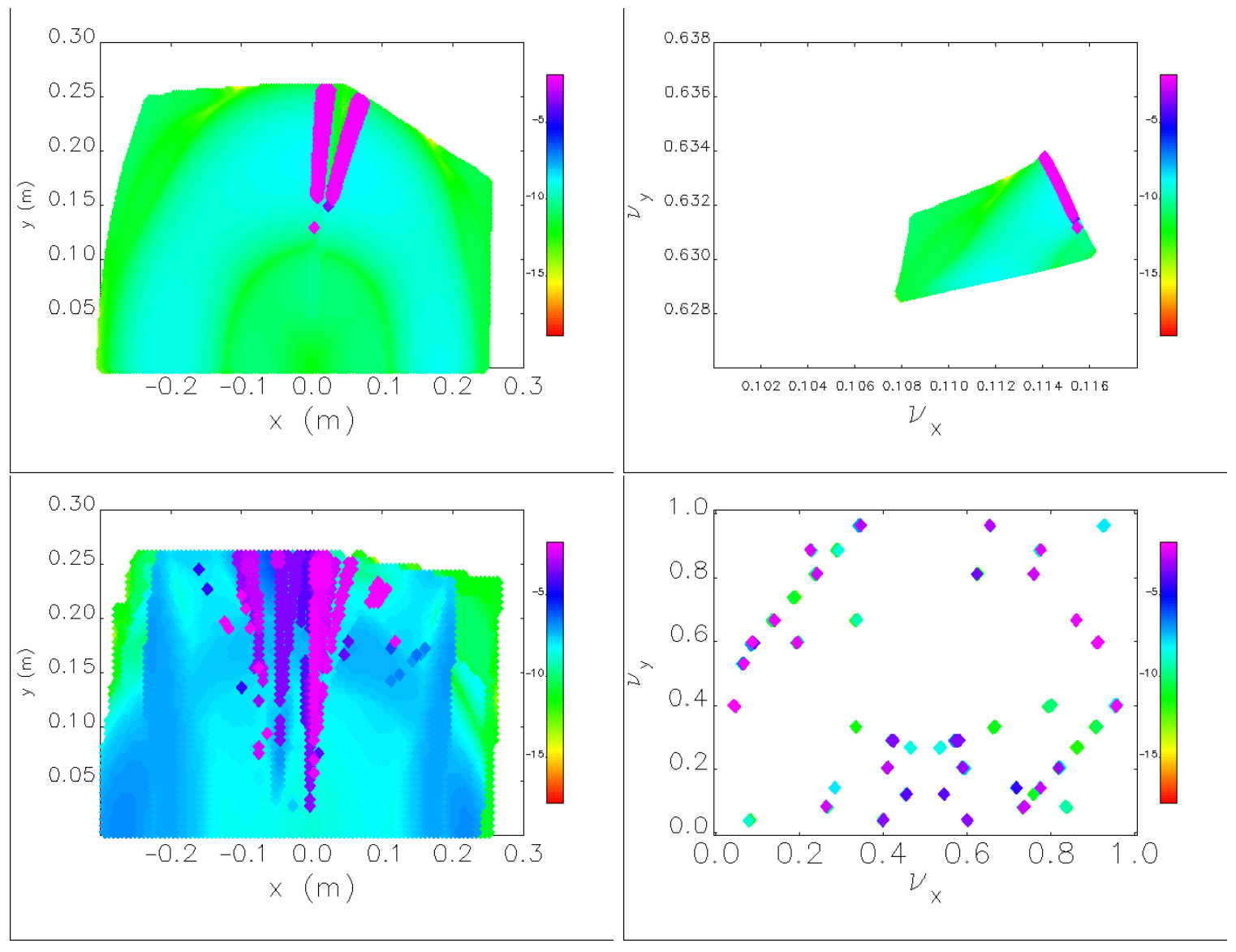

Figure 5.3: The FMA of the post-sextupole optimization ring design with combined function dipoles in the arcs in the $\mathrm{X}-\mathrm{Y}$ space (left) and on the betatron tune plane (right), on-momentum particles (upper) and off-momentum particles within the full range of $\delta= \pm 0.1$ (lower) are analyzed. 

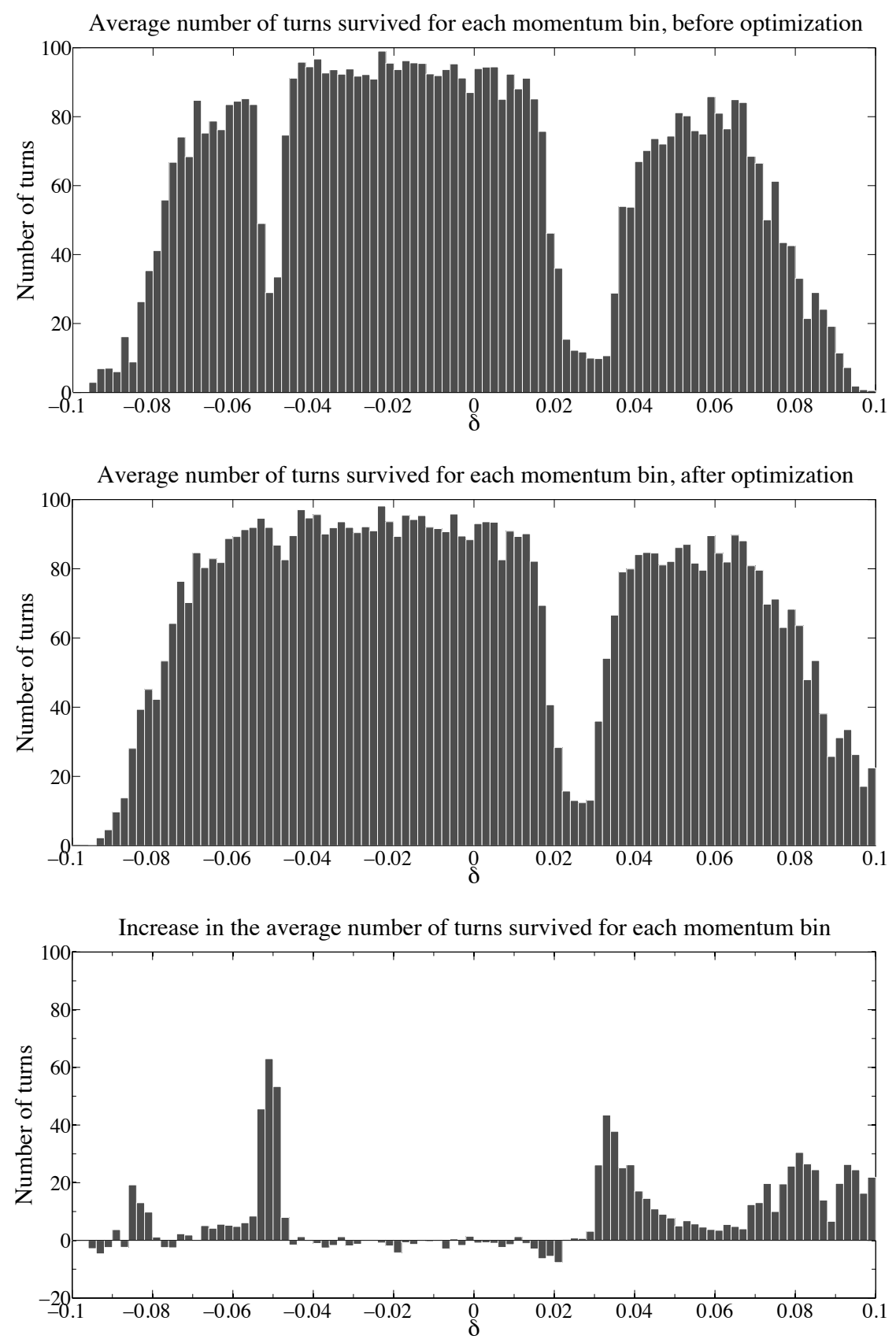

Figure 5.4: The average number of turns that particles in each momentum bin survive. The data were obtained from the tracking of $10^{5}$ particles, with $2 \mathrm{~mm} \cdot \mathrm{rad}$ transverse emittance and a momentum spread of $\delta \in \pm 10 \%$. The upper, middle, and lower plots correspond to data from the pre-optimized lattice, post-optimized lattice, and their difference (post minus pre). 
by higher-order chromatic effects, such as the terms in Table 5.2. The algorithm, while not able to correct all the nonlinear effects, finds the optimum configuration in a global manner.

It is seen from Figure 5.5 that the increase in acceptance for different emittance bins is independent of the emittance. Therefore, the optimization is more limited by the large momentum spread of the beam. The effectiveness of the optimization for muon beams with different admittance and momentum spreads is demonstrated in Figure 5.6. The acceptance of the pre-optimized ring, represented by the survival rate from $10^{4}$ initial particles after 100 turns (different random seeds were used to generate the $10^{4}$ particles), is shown by the upper plot. The acceptance of the postoptimized ring is shown by the lower plot as a comparison. From the lower plot, the optimization is most effective when both the emittance and the momentum spread are large. Moreover, it is more effective for a beam with larger momentum spread (compare the ratio vertically), than for a beam with larger admittance (compare the ratio horizontally). All of the analyses agree on this.

\section{Sextupole correction optimization for other beams}

In order to further understand the heuristic algorithm, another two optimization searches were performed. In these two scenarios, the transverse admittance and the momentum spread of the initial beam were reduced to $1 \mathrm{~mm} \cdot \mathrm{rad}$ and $\pm 5 \%$, respectively. It is observed from these two cases that the way a heuristic algorithm improves the acceptance is by a compromise over many nonlinearities. When the beam has smaller admittance, or narrower momentum spread, it is easier for the algorithm to find a good solution, since there are fewer nonlinearities to be corrected by the sextupoles. It is seen that the optimum correction scheme for either of these two cases 

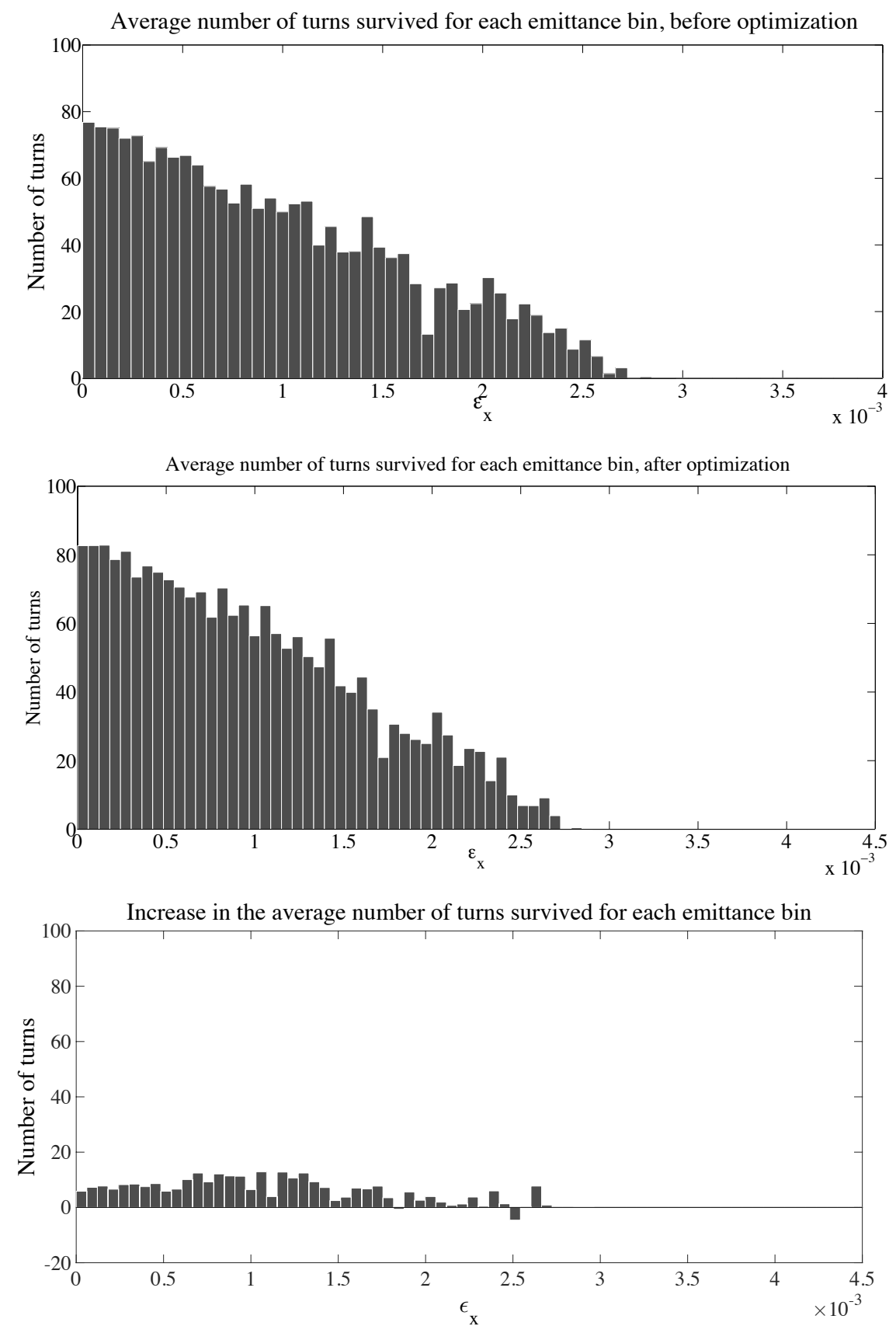

Figure 5.5: The average number of turns that particles survive for each $\epsilon_{x}$ bin. The data were obtained from tracking of $10^{5}$ particles, with $2 \mathrm{~mm} \cdot$ rad transverse admittance and a momentum spread of $\delta \in \pm 10 \%$. The upper, middle, and lower plots correspond to data from the pre-optimized lattice, post-optimized lattice, and their difference (post minus pre). 
Acceptance v.s. admittance and $\delta$, no correction

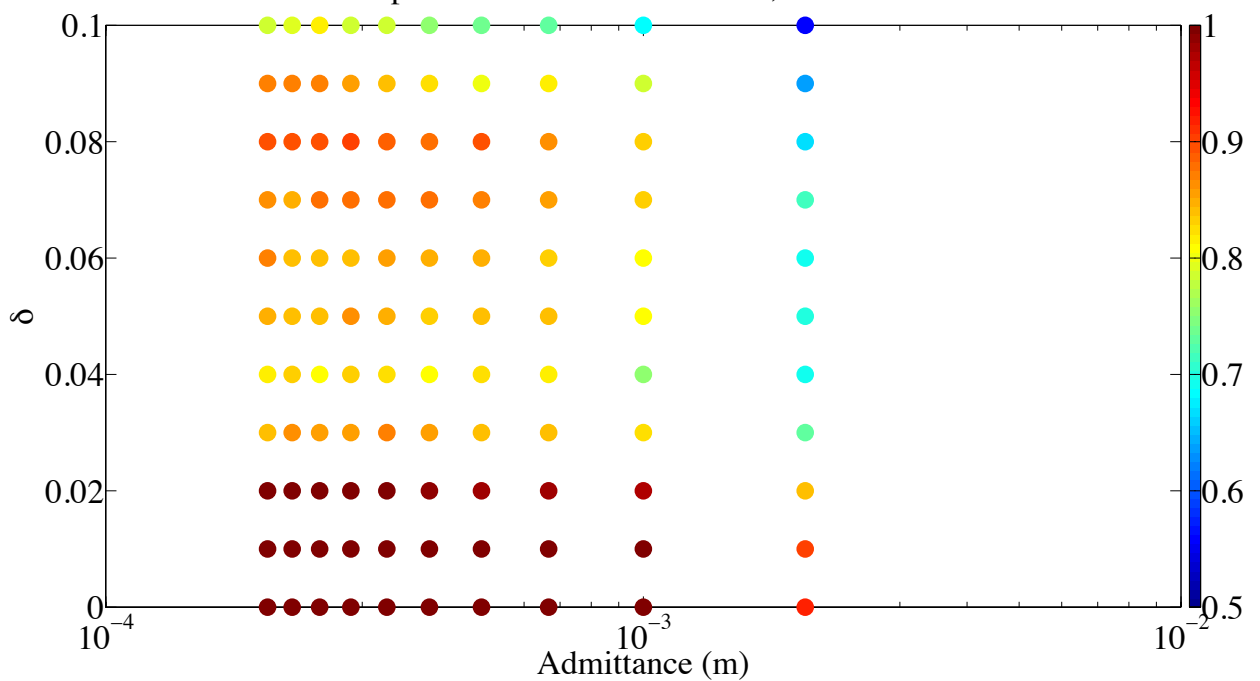

Admittance (m)

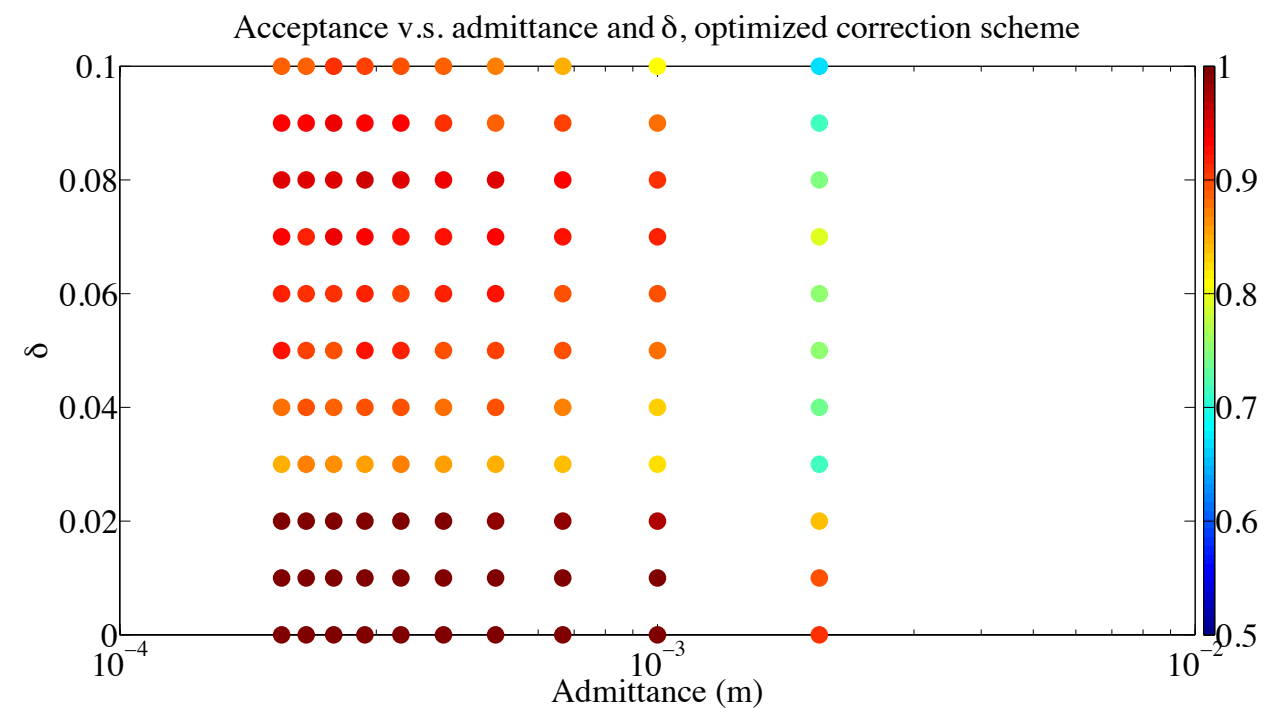

Figure 5.6: The effectiveness of the sextupole correction optimization. Upper: the acceptance of different initial beams without optimization; Lower: the acceptance of different initial beams with an optimized lattice. 
is not necessarily the best for the original case.

For a beam with $1 \mathrm{~mm} \cdot \mathrm{rad}$ transverse admittance, and a $\pm 10 \%$ momentum spread, the optimization was able to increase the acceptance of the beam from $71 \%$ to $86 \%$. The improvement on the average number of survival turns from the optimization is plotted in Figure 5.7. With smaller admittance, the beam has less nonlinearity from particles with large action, and it is easier to focus on the nonlinear effects induced by off-momentum particles. However, the stopbands at $\delta \sim-0.1$ and $\sim 0.03$ still produce strong nonlinearities and are not well corrected due to constraints from other nonlinearity sources. The effect of this correction scheme is also compared with the original correction shown in Figure 5.6 by plotting the ratios of the acceptance of the two corrected lattices to the linear lattice in Figure 5.8

For a beam with $2 \mathrm{~mm} \cdot$ rad transverse admittance, and a $\pm 5 \%$ momentum spread, the optimization search was able to increase the acceptance of the beam from $70 \%$ to $80 \%$. The increases in the average number of turns for the particles in each momentum and emittance bin are shown in Figure 5.9. There is still a stopband at $\delta \sim 0.03$, which suggests that the optimization found a good compromise by correcting other nonlinearities. The comparison of this correction scheme and the original correction scheme is shown in Figure 5.10.

Last but not the least, in order to investigate the momentum stopband at $\delta \sim 0.03$, a special test beam, with $2 \mathrm{~mm} \cdot \mathrm{rad}$ transverse admittance and $\delta \in[0.02,0.04]$ was generated for tracking. The optimization was run again to find the optimum correction scheme specifically for this beam, which indicates whether it is possible for the previous optimizations to avoid the stopbands. The acceptance for this beam was increased from only $16 \%$ to $88 \%$ by the algorithm. As a comparison, the average number of survived turns for particles in each momentum bin, corresponding to 

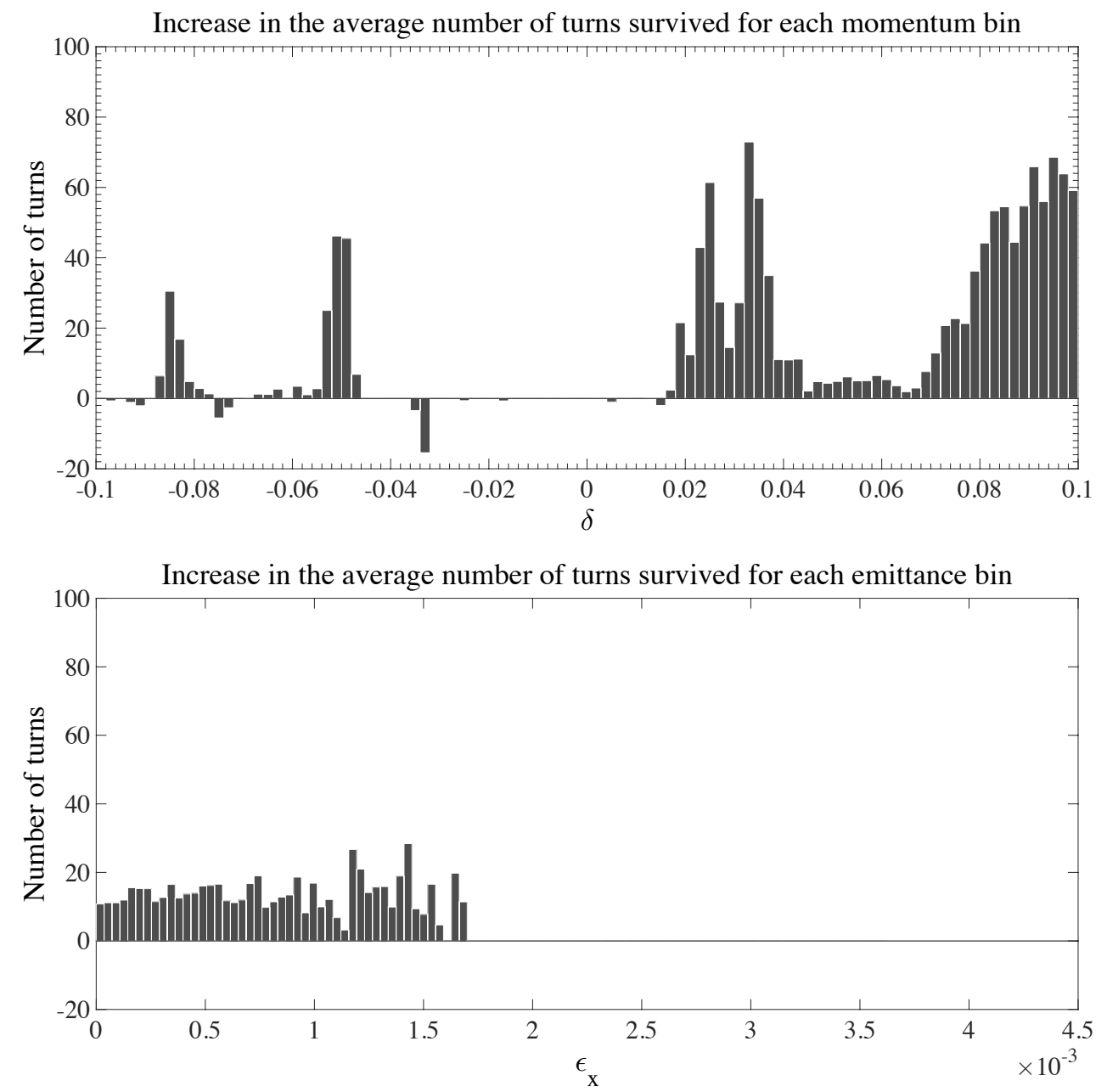

Figure 5.7: The increase in the average number of turns that particles survive for each momentum and $\epsilon_{x}$ bin. The data were obtained from tracking of $10^{5}$ particles, with $1 \mathrm{~mm} \cdot \mathrm{rad}$ transverse admittance and a momentum spread of $\delta \in \pm 10 \%$. 


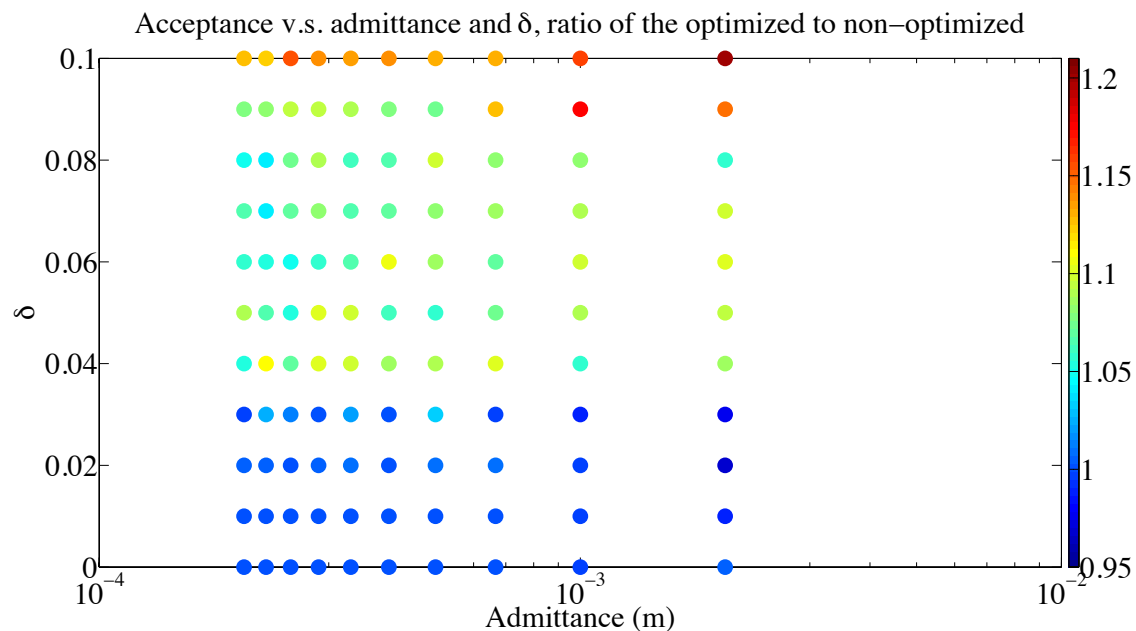

Acceptance v.s. admittance and $\delta$, ratio of the optimized to non-optimized

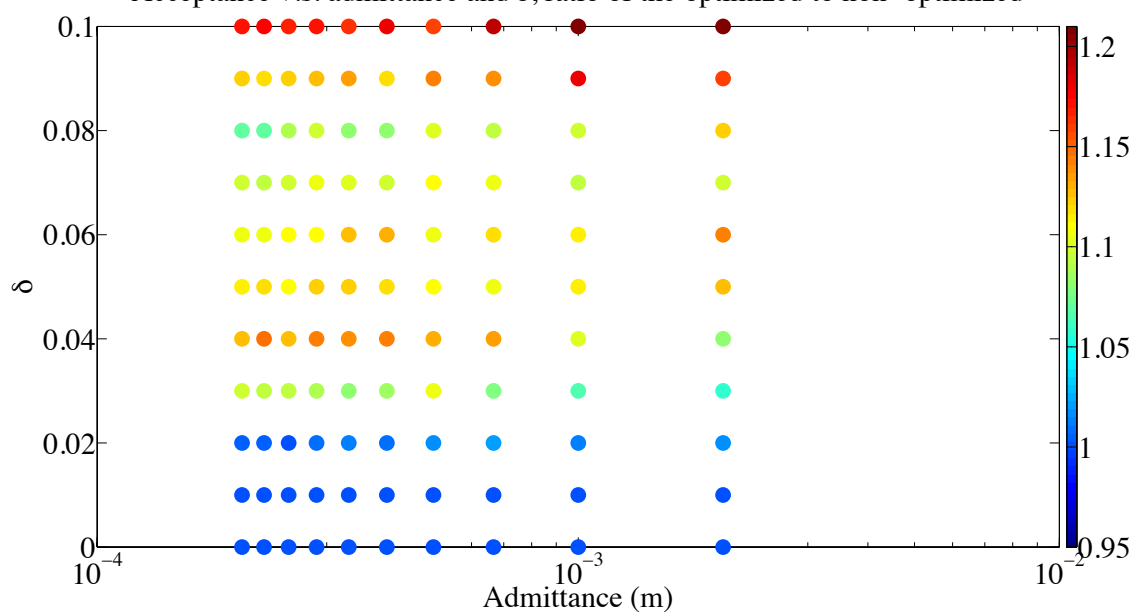

Figure 5.8: The increase in acceptance of different beams, represented by the ratio of the acceptance of the optimized lattices to the linear lattice. Upper: the original optimized lattice; Lower: the optimized lattice for a beam with $1 \mathrm{~mm} \cdot \mathrm{rad}$ transverse admittance and a momentum spread of $\delta \in \pm 10 \%$. 

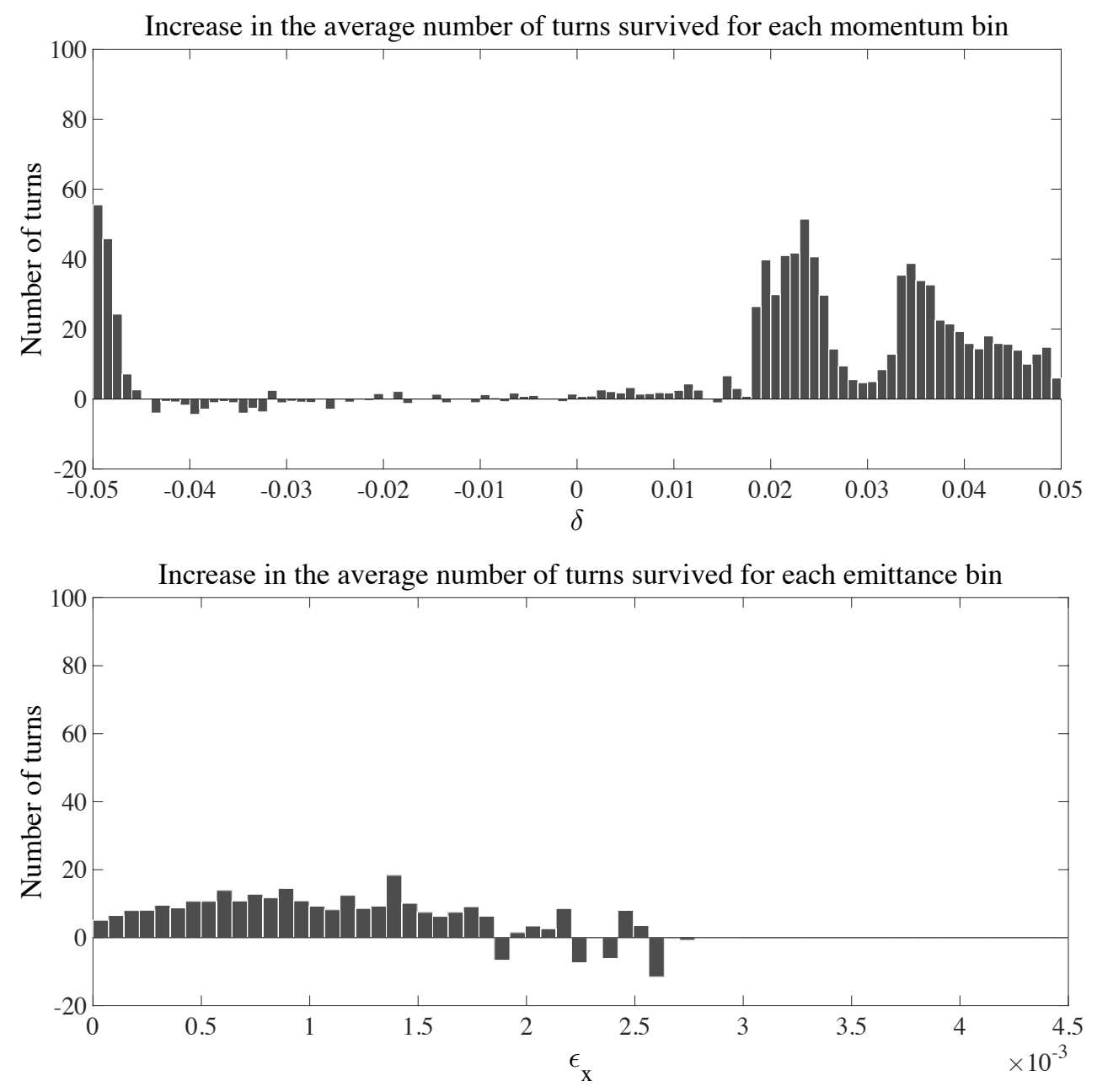

Figure 5.9: The increase in the average number of turns that particles survive for each momentum and $\epsilon_{x}$ bin. The data were obtained from tracking of $10^{5}$ particles, with $2 \mathrm{~mm} \cdot \mathrm{rad}$ transverse admittance and a momentum spread of $\delta \in \pm 5 \%$. 


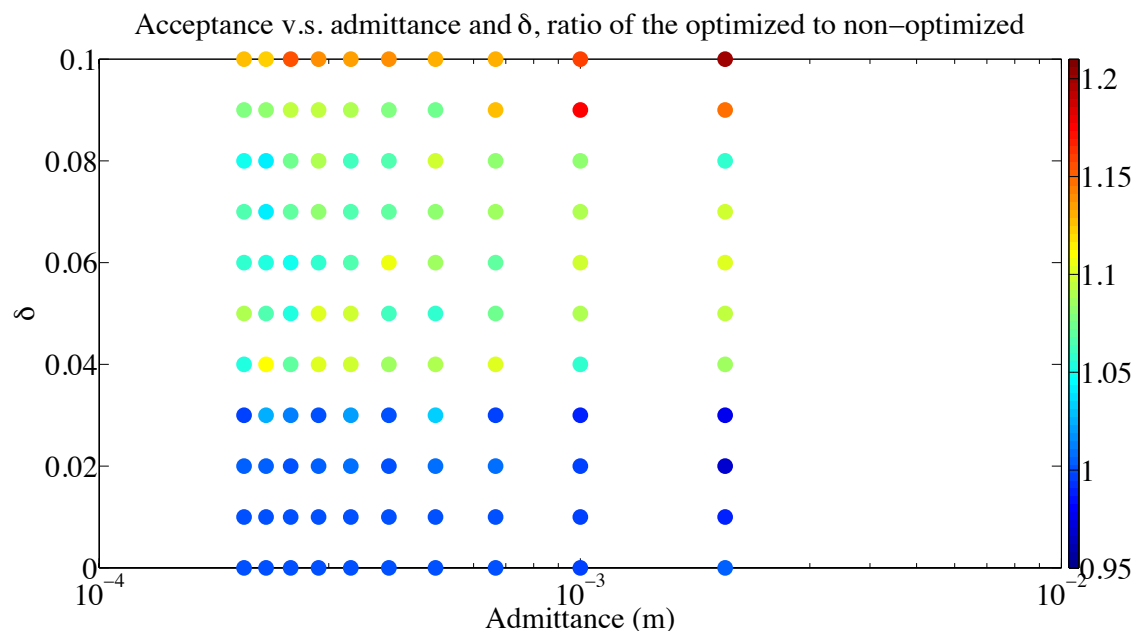

Acceptance v.s. admittance and $\delta$, ratio of the optimized to non-optimized

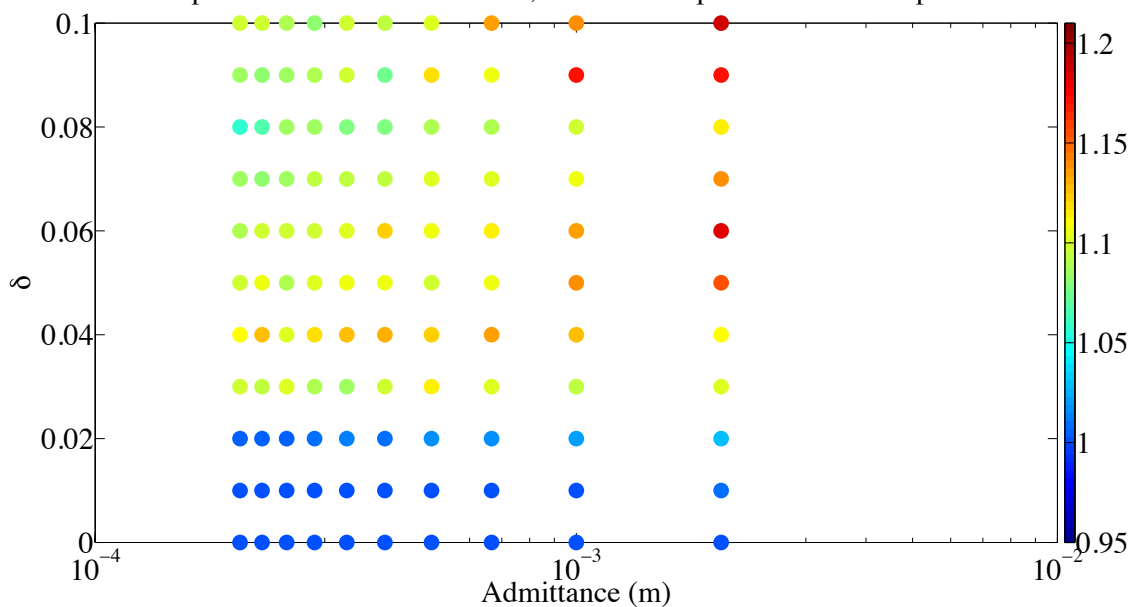

Figure 5.10: The increase in acceptance of different beams, represented by the ratio of the acceptance of the optimized lattices to the linear lattice. Upper: the original optimized lattice; Lower: the optimized lattice for a beam with $2 \mathrm{~mm} \cdot \mathrm{rad}$ transverse admittance and a momentum spread of $\delta \in \pm 5 \%$. 
Figure 5.4, is plotted in Figure 5.11

It can be seen that, the stopband at $\delta=0.03$ was successfully corrected by the optimization. The acceptance of this beam is limited by its large admittance, as the nonlinearities induced by the momentum have been corrected to get a uniform momentum acceptance (the middle plot). However, in order to make this correction, other nonlinearities are increased and reduce the acceptance of the full-sized beam, which is the reason why the optimization shown by Figure 5.11 was not obtained with a full-sized beam. The comparison of this correction scheme and the original correction scheme is shown in Figure 5.12. The low acceptance of the full-sized beam (upper-right points) confirms that the correction of this momentum stopband creates other nonlinearities and thus is not a globally optimum configuration.

\subsubsection{Arc design with conventional, non-superconducting magnets Linear Optics and the FMA}

Another possible design option was proposed to avoid the complexity of introducing cryogenic equipment into the facility. The arc was redesigned to include only conventional, combined-function dipoles. The parameters of the new design option are listed in Table 5.3, and the linear optics are plotted in Figure 5.13.

Compared that with superconducting dipoles in the arcs, the new design has a bigger circumference (536 compared to 488), and requires more combined function dipoles (24 compared to 13). The drift space between magnets is also too small for individuals sextupoles to be placed, therefore, the sextupole correction was added only to the combined-function dipoles to make them multipoles, which still keeps the pole-tip field below 2 Tesla.

The heuristic algorithms were applied again to the sextupole optimization. The 

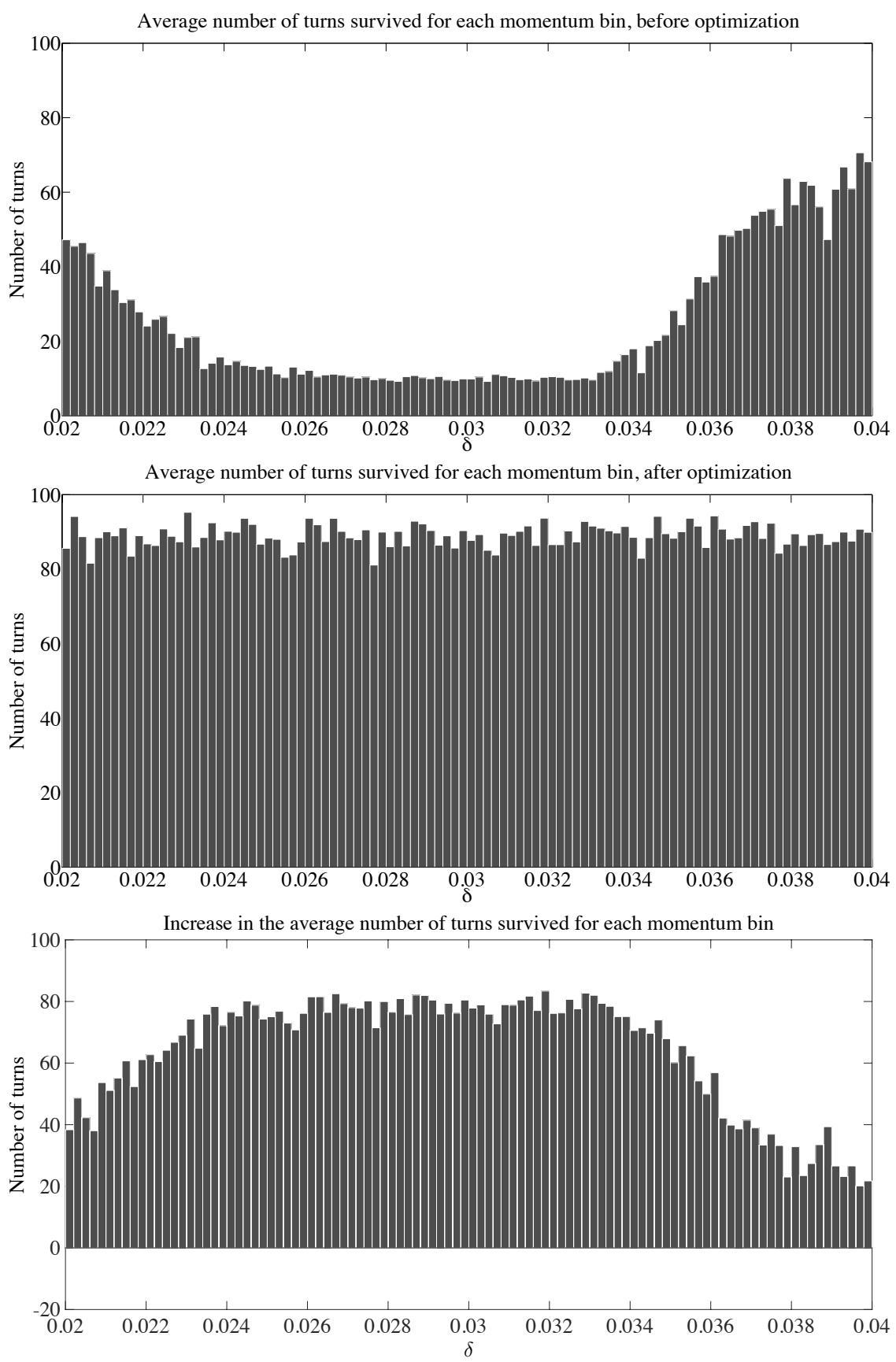

Figure 5.11: The average number of turns that particles in each momentum bin survive. The data were obtained from tracking of $10^{5}$ particles, with $2 \mathrm{~mm} \cdot \mathrm{rad}$ transverse emittance and a momentum spread of $\delta \in[0.02,0.04]$. The upper, middle, and lower 117

plots correspond to data from the pre-optimized lattice, post-optimized lattice, and their difference (post- minus pre-). 

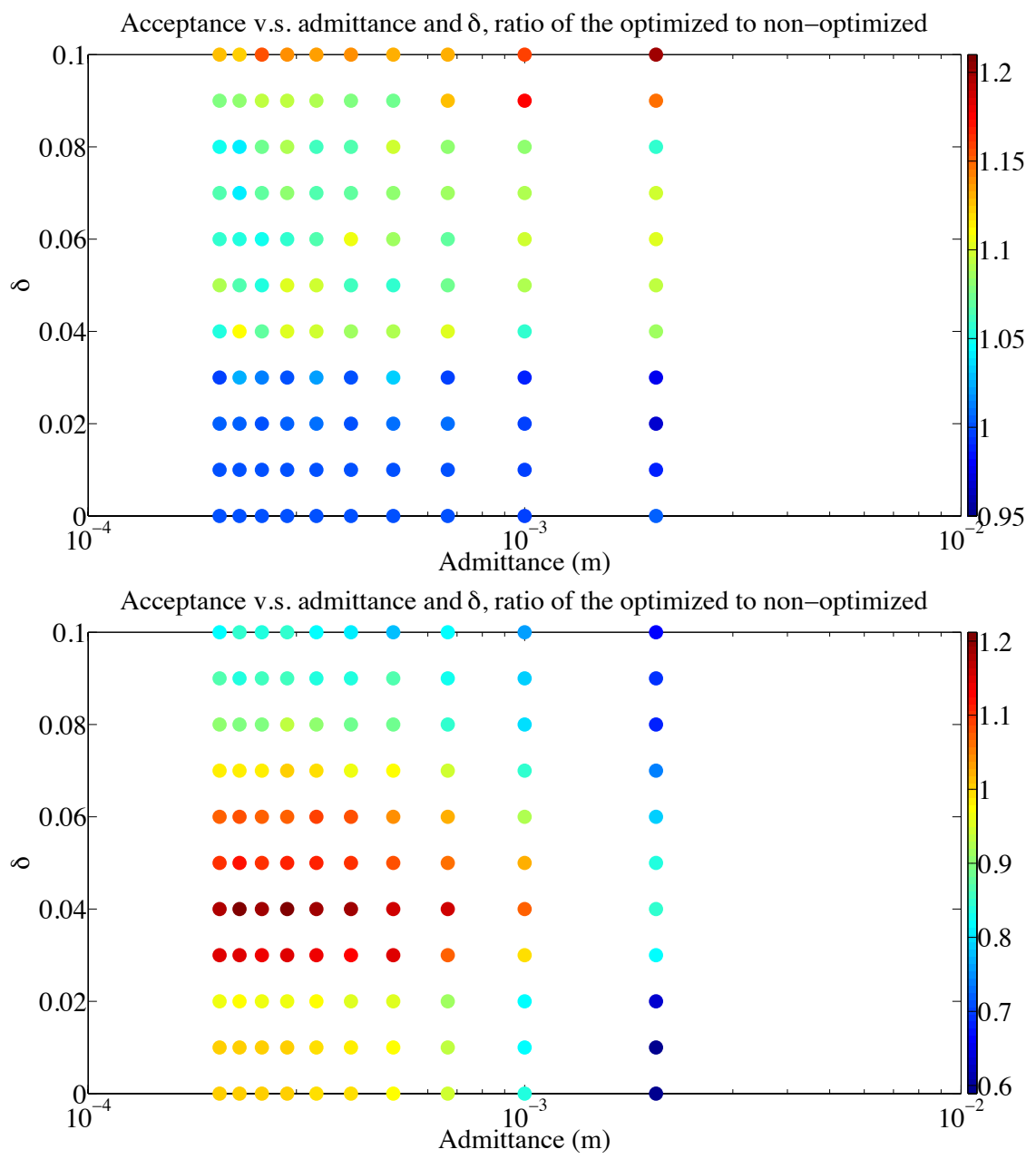

Figure 5.12: The increase in acceptance of different beams, represented by the ratio of the acceptance of the optimized lattices to the linear lattice. Upper: the original optimized lattice; Lower: the optimized lattice for a beam with $2 \mathrm{~mm} \cdot \mathrm{rad}$ transverse admittance and a momentum spread of $\delta \in[0.02,0.04]$. 


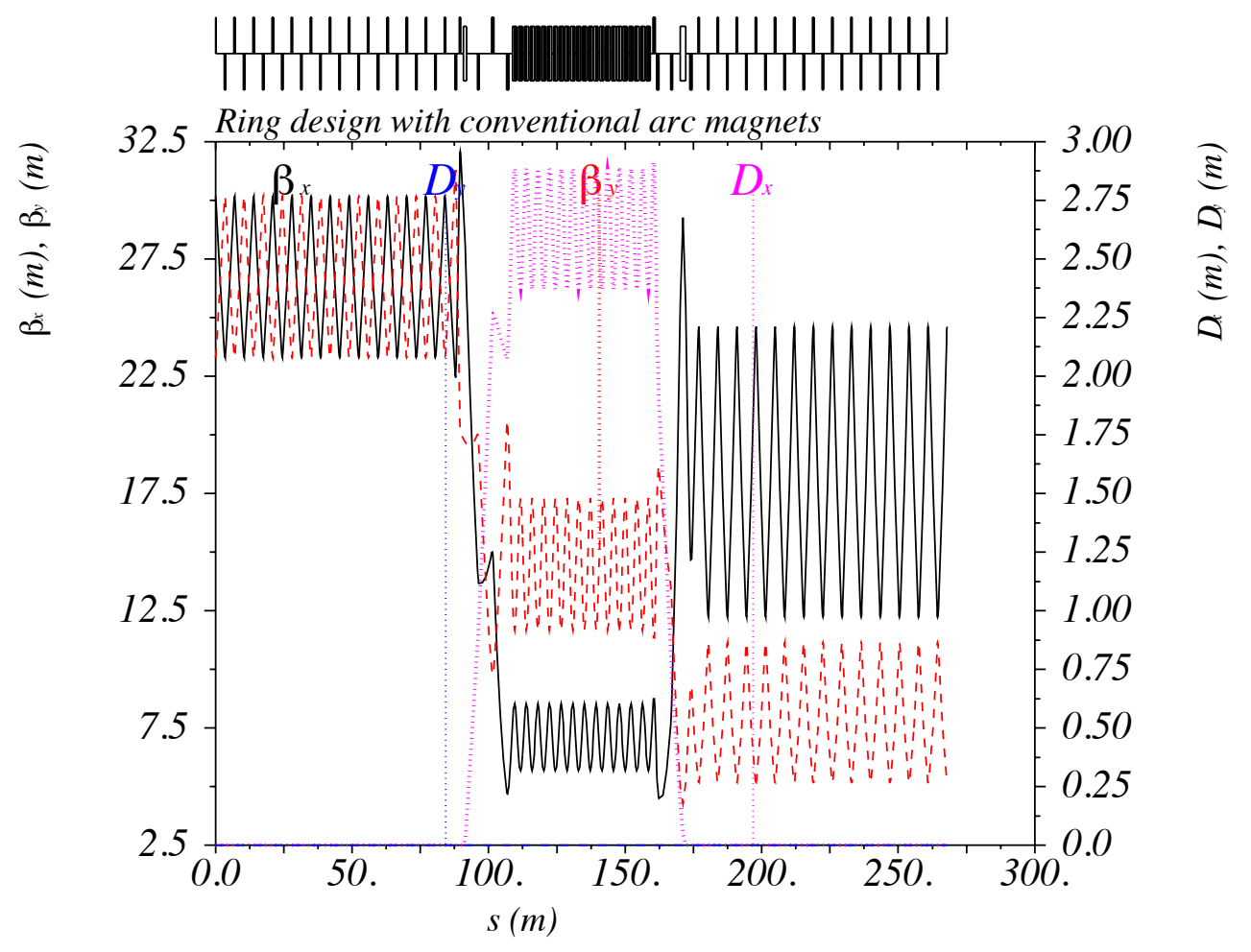

Figure 5.13: The linear optics of a nuSTORM decay ring design with conventional combined function dipoles in the arcs. $\beta_{x}$ : black, $\beta_{y}$ : red, $D_{x}$ : green. 
Table 5.3: Parameters of the decay ring design with non-superconducting arc dipoles

\begin{tabular}{|l|l|}
\hline Parameters & Values $($ units $)$ \\
\hline \hline Central momentum $P_{0, \mu}$ & $3800(\mathrm{MeV} / \mathrm{c})$ \\
Circumference & $535.9(\mathrm{~m})$ \\
Arc length & $86.39(\mathrm{~m})$ \\
Straight length & $181.56(\mathrm{~m})$ \\
$\left(\nu_{x}, \nu_{y}\right)$ & $(6.23,7.21)$ \\
$\left(d \nu_{x} / d \delta, d \nu_{y} / d \delta\right)$ & $(-3.11,-12.73)$ \\
\hline
\end{tabular}

acceptance of a full-sized beam without sextupole correction is 50\%, while the optimized correction configuration increases the acceptance to 57\%. The optimization was run four times with independent initial populations, each time converging to the same optimized acceptance. The average number of turns survived for particles in each momentum bin is plotted in Figure 5.14. The big loss at large $|\delta|$ locations suggest that this design is greatly limited by the chromatic effects, for which the correction is either not possible under the constraints, or will create other nonlinearities that destroy the global acceptance of the full-sized beam.

Other options were also tried in the design, such as a ring with Double Bend Achromat (DBA) arcs, and a "D-shape" ring where there is only one straight section. These designs either yield a very low acceptance of the full-sized beam, even with optimized sextupole correction, or have very large circumferences. Therefore those details are omitted in this dissertation.

\subsection{Neutrino flux at nuSTORM}

In this section, the neutrino beams produced by the nuSTORM facility are described. Since the parent particle distribution can be precisely measured using conventional 

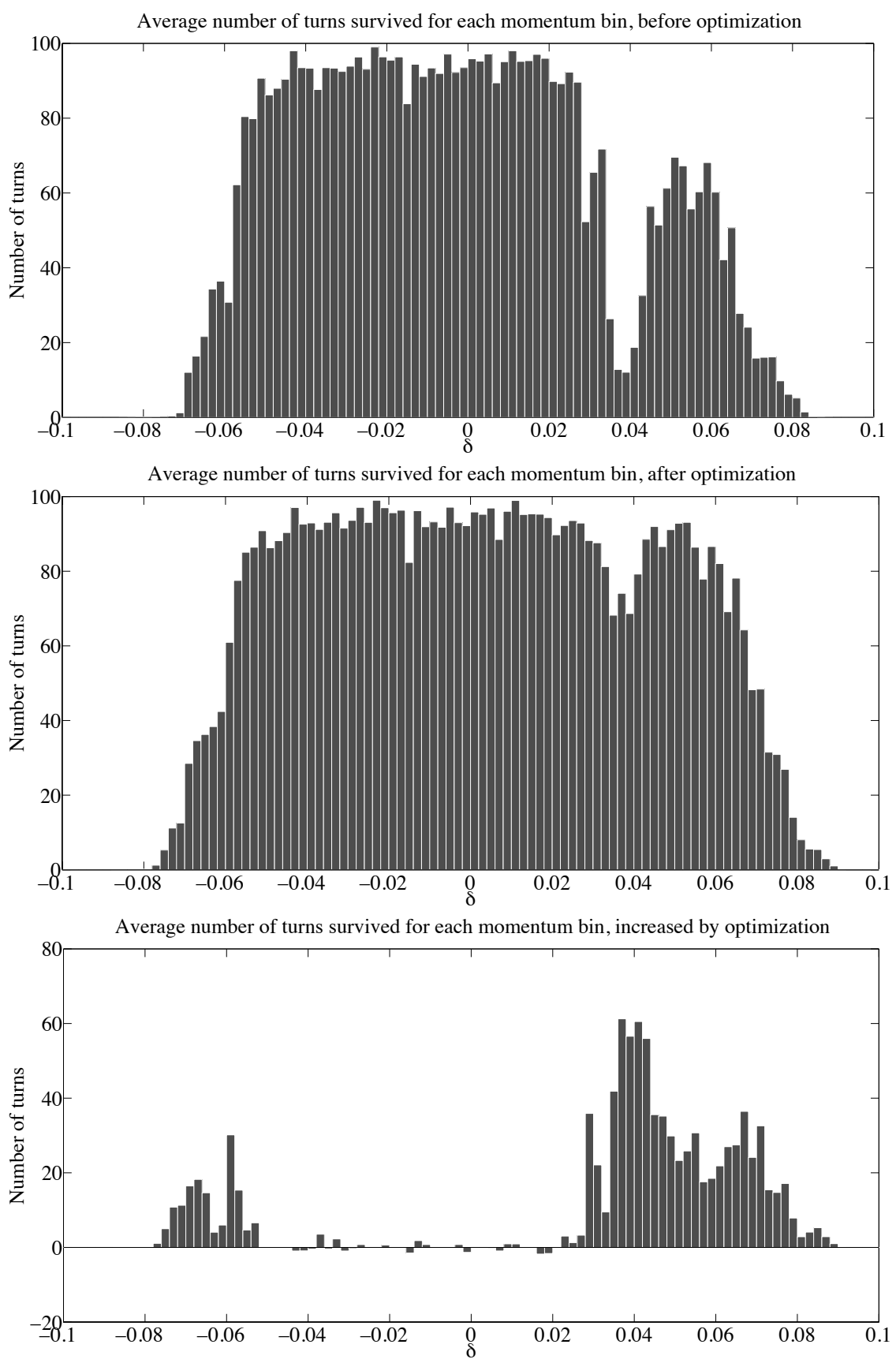

Figure 5.14: The average number of turns that particles in each momentum bin survive. The data were obtained from tracking of $10^{5}$ particles, with $2 \mathrm{~mm} \cdot \mathrm{rad}$ transverse emittance and a momentum spread of $\delta \in \pm 10 \%$. The upper, middle, and lower plots correspond to data from the pre-optimized lattice, post-optimized lattice, and their difference (post minus pre). 
beam diagnostic instrumentation, the neutrino beams can be determined with excellent precision. Although nuSTORM is primarily designed to produce neutrinos from circulating muons, the pion beamline design (Chapter 3) also produces an intense $\overrightarrow{\nu_{\mu}}$ beam from $\pi^{ \pm}$decay in the production straight, during injection. The neutrino flavor composition and the expected bin-to-bin errors are presented in this section.

\subsubsection{Neutrino flux from the pion beam}

To quantitatively investigate the neutrino beams, all the secondary particles obtained at the downstream end of the target (discussed in Section 3.5.2) are tracked in the pion beamline. The ensemble of all particle types are sampled at fifty locations along the production straight to generate an ensemble of particles representative of the beam. As the decay kinematics are well known, the sampled ensemble can be used to calculate the neutrino flux at any arbitrary location. G4BL can also produce the neutrino flux from the beam by forcing the particles to decay instantly, but statistics will be limited by the suppressed decay branches. The sampling is considered to be analogous to the beam information obtained by standard beam diagnostics. The calculated neutrino flux from the sampled ensemble can be seen in Figure 5.15 [3]. The pion beamline is tuned to transport $\pi^{+}$in this simulation. The results are normalized

to $10^{21} 120 \mathrm{GeV}$ POT. The decay branches $K^{+} \rightarrow \nu_{e}, \pi^{-} \rightarrow \bar{\nu}_{\mu}$, and $\pi^{+} \rightarrow \nu_{e}$ are included in the calculation, but their contributions to the flux are too small to be seen on the scale of the plots.

\subsubsection{Neutrino flux from the muon beam}

The muons are treated in a similar manner to get a sampled beam along the production straight for neutrino flux calculations. The neutrino flux at the ND and FD from 

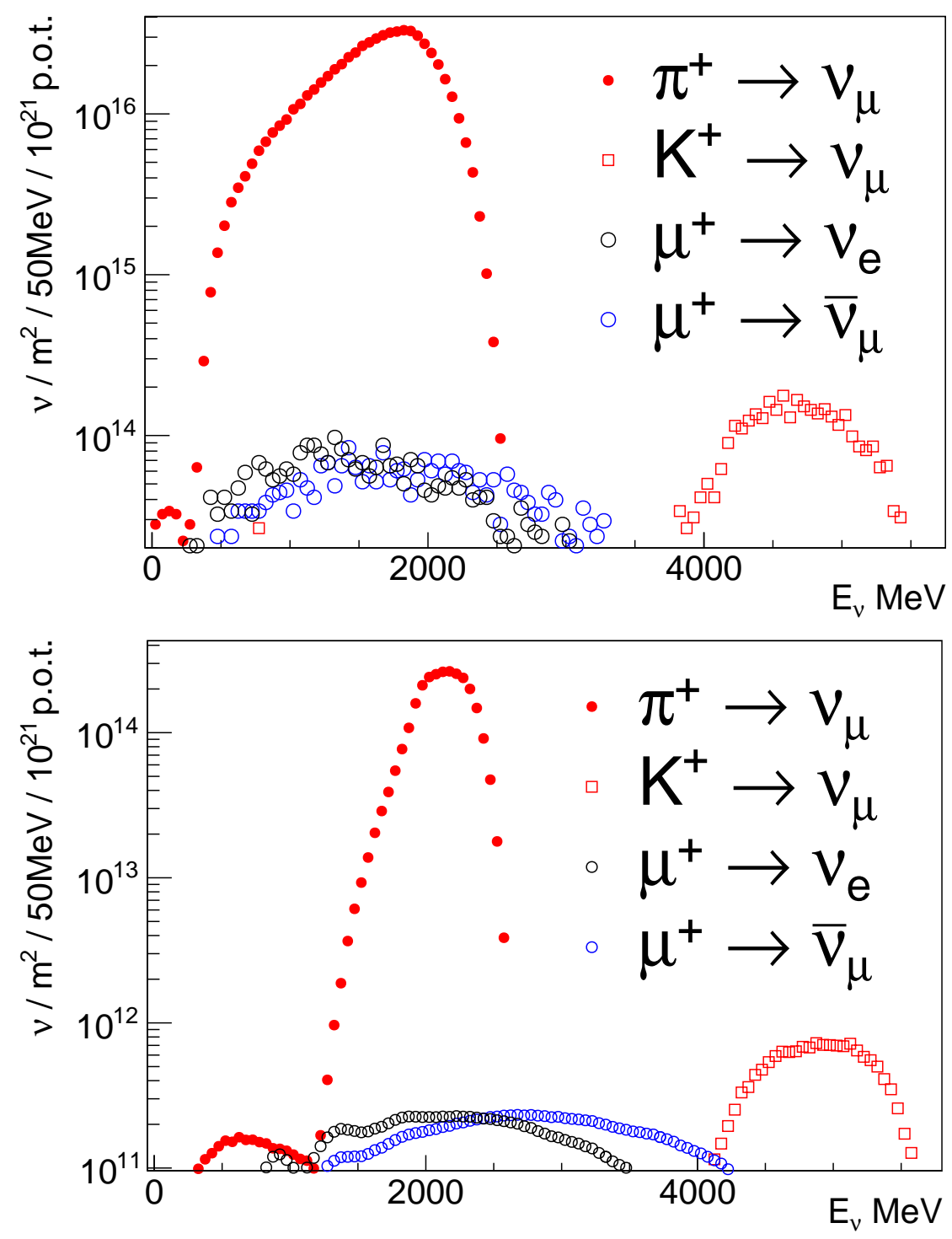

Figure 5.15: Neutrino flux from the sampled injection beam of all particle types, normalized to $10^{21} 120 \mathrm{GeV}$ POT. The top and bottom plots correspond to the flux at the near $(50 \mathrm{~m})$ detector and far $(2000 \mathrm{~m})$ detector, respectively. 
the $\mu^{+}$beam can be seen in Figure 5.16. It is expected that the errors on the binned flux are dependent only on the diagnostics-determined particle trajectories. In order to evaluate the effect of measurement errors on the muon divergence in the ring, the divergence of each particle was inflated by $2 \%$ compared to the nominal value. With this modified beam, the mean flux difference in the $50 \mathrm{MeV}$ neutrino energy bins is determined to be $\approx 0.6 \%$. Beam Current Transformers (BCTs) have the potential to measure the circulating muon intensity to a precision of roughly $0.1 \%$. With all factors considered, the flux bin errors will be below $1 \%$.

\subsection{Summary}

The nuSTORM muon decay ring aims to accept a muon beam with both a large transverse admittance of $2000 \mu \mathrm{m}$ and a large momentum range of $3.8 \pm 10 \% \mathrm{GeV} / \mathrm{c}$. It has been shown that the beam is greatly affected by the nonlinearities of the lattice, including chromatic detuning, geometric detuning, and nonlinear dispersions, etc. Due to the constraints on the ratio of arc section lengths to straight section lengths, the sextupole correction for the nonlinearities is limited by the number of sextupoles or sextupolar fields that can be placed in the ring and their strengths.

Two ring designs, one with arc cells formed by superconducting combined function dipoles and the other one with conventional magnets have been shown and compared. From a multi-particle tracking simulation in MAD-X, in which the beam was normally distributed in the $2 \mathrm{~mm} \cdot \mathrm{rad}$ admittance and uniformly distributed in $3.8 \pm 10 \% \mathrm{GeV} / \mathrm{c}$, $58 \%$ and $50 \%$ of the particles survive after circulating by 100 turns. The sextupole correction scheme for each design was optimized with both the genetic algorithm and the simulated annealing algorithm, for which the results converge for the two heuristic Monte Carlo methods. 
It has been shown that the algorithms can find and react to the dominant nonlinear terms, but may not be able to correct all the nonlinearities within all the constraints, which include the number of sextupolar fields that can be placed, the maximum strength of the fields, and the position of the sextupole corrections, and so forth. With the optimization, the acceptance of the designs is increased to $67 \%$ and $57 \%$, respectively. The efficiency of the optimization was confirmed by more test runs of the algorithm, but with different beams to be accepted by the optimized beamline. It was shown that the heuristic algorithms can find the best balance among all the dominant nonlinearities for each beam, and find the global optimum of the problem. An example was given, in which one momentum stopband was corrected by the optimization, and which shows that the algorithm will not fall into the correction for a single nonlinearity if the correction creates other destructive nonlinearities. Feasibility of correction schemes with higher-order multipoles can be further investigated when the large aperture magnets are fully studied with design and simulations.

The nuSTORM facility is capable of producing intense $\stackrel{\stackrel{c}{\nu}}{\mu}$ beams from pion decay and $\stackrel{\mathrm{\nu}}{\nu}_{e}+\stackrel{\mathrm{c}}{\nu}_{\mu}$ beams from muon decay. It has been shown that the beamline provides bright and flavor-pure neutrino beams that can be precisely determined by the beam diagnostics implemented in the pion beamline and the decay ring. The low background contributes to a high confidence level $(10 \sigma)$ that a $\nu_{\mu}$ appearance measurement alone can provide a definitive statement about the existence of a sterile neutrino in the region where the combination of the fit to the LSND, MiniBooNE, gallium, and reactor data suggest such oscillations exist. 

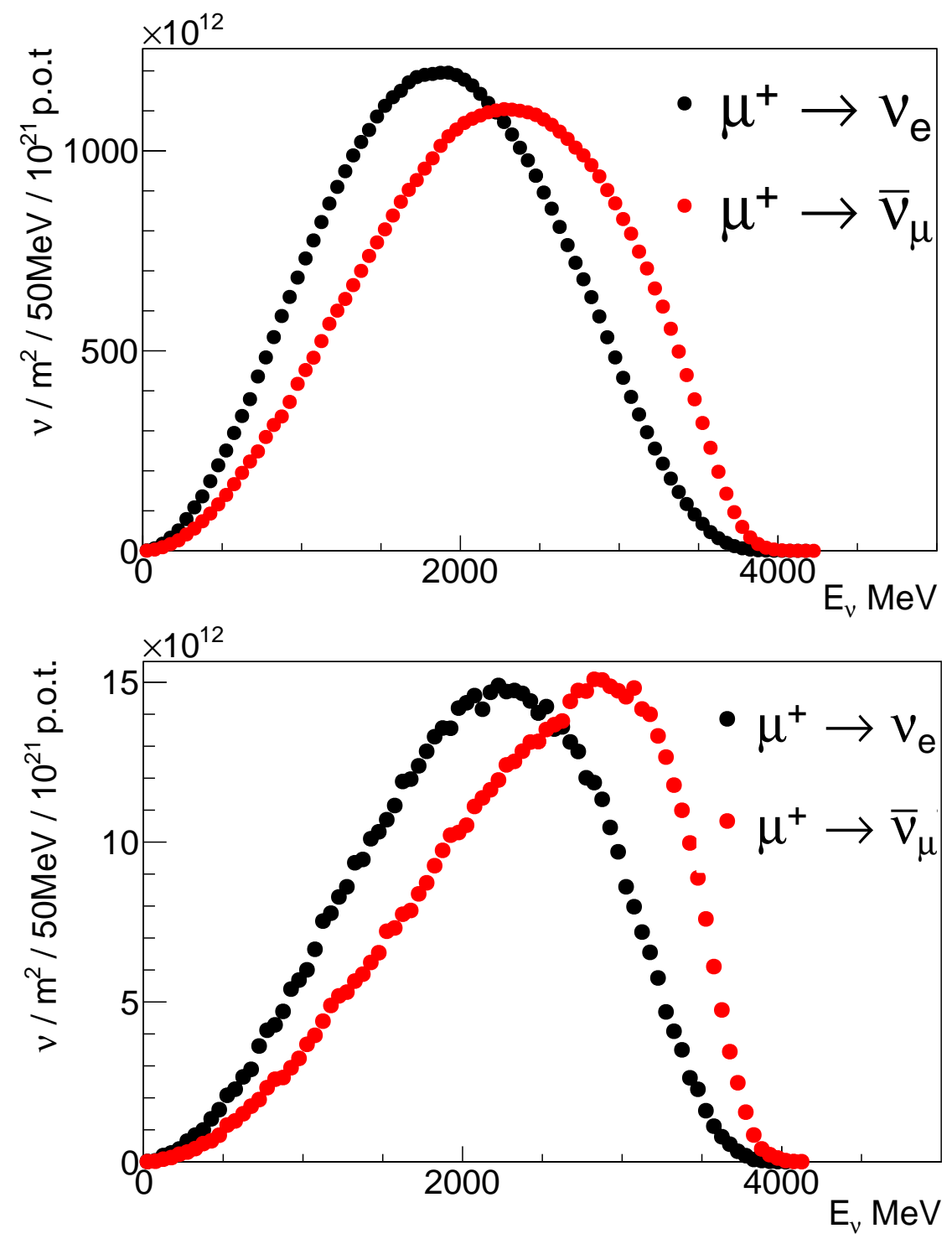

Figure 5.16: Neutrino flux from the sampled muon beam along the production straight, normalized to $10^{21} 120 \mathrm{GeV}$ POT. The top and bottom plots correspond to the flux at the near detector and far detector, respectively for the beam in the first 1.6 


\section{CHAPTER 6}

\section{CONCLUSIONS}

In this dissertation, the design of the nuSTORM facility including the magnetic collection horn, the pion beamline and the muon storage ring is discussed. The design is of essential importance to the demonstration of the stochastic injection scenario and future muon facilities. The dissertation also includes the detailed tracking of the beams produced at nuSTORM with Monte Carlo simulations of particle decays in the beamline. In addition, the design of the horn and the decay ring are optimized by modified and improved heuristic algorithms.

The design of the pion beamline and the stochastic injection scheme is demonstrated. The pion beamline is designed to transport pions from the downstream end of the horn to the end of the production straight of the decay ring, and accept the muons from pion decays in the production straight. The production straight has FODO optics matched to periodic oscillations for both the pion and muon beams, which have different momenta. The optics of the pion beamline is matched backward from the FODO cells to the collection horn. In order to implement the stochastic injection, which avoids the use of a fast kicker and a septum with very large apertures, the Orbit Combination Section (OCS) was designed to combine the two design orbits at injection. This is primarily done by having a large dispersion at the injection 
point, and suppressing it to zero using the combination of a defocusing quadrupole and a dipole. The non-dispersive beam from the horn is matched, to obtain the same optics at the injection point, by the dipoles in the pion transport line. The dipoles in the pion beamline not only match the beam optics, but also select the pions in momentum and charge. The remaining high energy $120 \mathrm{GeV}$ protons that are not transmitted by the first dipole are directed to a beam absorber downstream of the dipole. The performance of the pion beamline is examined by tracking the beam in G4Beamline. Within the desired ring acceptance, 0.011 muons per POT are delivered to the end of the production straight with this design.

In order to increase the muon flux in the ring, the nuSTORM horn was optimized by an enhanced multi-objective genetic algorithm with MPI enabled. The optimization objectives are derived by analyzing the pion decay kinematics and the acceptance of the pion beamline. The conversion from single objective to multi-objective eliminates tracking the beam through the beamline, so that the fitness values for each horn can be calculated readily after the pions are tracked through the magnetic field of the horn. The optimizations are carried out for Inconel targets with different lengths. It is shown from the results that the number of muons in the ring acceptance is increased by $16 \%$ when a $46 \mathrm{~cm}$ Inconel target and the corresponding optimal horn are used, compared to the baseline $38 \mathrm{~cm}$ Inconel target and horn. The application of the technique in this optimization study is not limited to either the nuSTORM facility or muon based facilities in general, but can be used on other neutrino experiments that use magnetic horns as collection devices.

The muon beam in the nuSTORM decay ring occupies a large transverse phase space area, and has a wide momentum spectrum. FODO rings with such a large acceptance have not been fully investigated prior to nuSTORM. Two designs with 
different features are proposed. Sextupoles or sextupolar fields are added to the lattices to correct the chromatic and geometric nonlinearities. Using a simulated annealing algorithm, the acceptance of both of the designs, defined as the percentage of the particles surviving 100 turns in the ring, is increased by more than $15 \%$. Using arc cells with combined function dipoles, we find that $67 \%$ of the muon beam can survive circulating the ring for 100 turns, without considering decays.

The neutrino flux at nuSTORM is calculated by sampling the beams in the production straight from a G4Beamline simulation. The momentum and charge selection in the nuSTORM beamline can reduce the background uncertainty by removing the particles that give undesired neutrino flavors. nuSTORM is capable of delivering flavor-pure and precisely measurable electron and muon neutrino beams, which can provide definitive statements about the existence of a sterile neutrino. 


\section{APPENDIX A \\ SELECTED SIMULATION AND ALGORITHM DETAILS}

\section{A.1 Gauss-Newton Algorithm}

The Gauss-Newton algorithm is used to find the minimum of an objective function,

which is a sum of squares. Therefore, it is useful in doing a nonlinear regression to fit the data to a multivariate function, e.g. to fit to a bivariate Gaussian function. The quantity the algorithm minimizes is

$$
Q(\boldsymbol{\alpha})=\sum_{i=1}^{n} r_{i}^{2}(\boldsymbol{\alpha})
$$

where $\boldsymbol{\alpha}$ is the vector of variables $\left(\alpha_{1}, \alpha_{2}, \ldots, \alpha_{M}\right)$, and $r_{i}$ can be any functions of $\boldsymbol{\alpha}$. If we write the $N$ residuals as a vector,

$$
\boldsymbol{r}(\boldsymbol{\alpha})=\left(\begin{array}{c}
r_{1}(\boldsymbol{\alpha}) \\
r_{2}(\boldsymbol{\alpha}) \\
\ldots \\
r_{N}(\boldsymbol{\alpha})
\end{array}\right)
$$

then the Gauss-Newton iterations are performed by iterationing

$$
\boldsymbol{\alpha}^{(s)}=\boldsymbol{\alpha}^{(s-1)}-\left(\boldsymbol{J}_{\boldsymbol{r}}^{T} \boldsymbol{J}_{\boldsymbol{r}}\right)^{-1} \boldsymbol{J}_{\boldsymbol{r}}^{T} \boldsymbol{r}\left(\boldsymbol{\alpha}^{(s-1)}\right)
$$

starting with $\boldsymbol{\alpha}^{(0)}$ as the initial guess. $\boldsymbol{J}_{\boldsymbol{r}}$ is the Jacobian matrix of $\boldsymbol{r}$ at $\boldsymbol{\alpha}^{(s-1)}$,

$$
\left(\boldsymbol{J}_{\boldsymbol{r}}\right)_{i, j}=\frac{\partial r_{i}\left(\boldsymbol{\alpha}^{(s-1)}\right)}{\partial \alpha_{j}}, \quad(i=1, \ldots, N ; j=1, \ldots, M)
$$




\section{A.1.1 Gauss-Newton in bivariate Gaussian fitting}

For fitting with nonlinear regression, the $r_{i}$ are the residuals defined by

$$
r_{i}(\boldsymbol{\alpha})=z_{i}-f_{i}(\boldsymbol{\alpha}) .
$$

where $f_{i}(\boldsymbol{\alpha})$ is the ith value given by the function and $z_{i}$ is the ith observation value. For bivariate Gaussian fitting, the theoretical probability density function $f_{i}(\boldsymbol{\alpha})$ is defined as,

$$
\begin{aligned}
f_{i}(\boldsymbol{\alpha}) & =f\left(\left(u_{i}, u_{i}^{\prime}\right), \boldsymbol{\alpha}\right) \\
& =\frac{1}{2 \pi \sigma_{u_{i}} \sigma_{u_{i}^{\prime}} \sqrt{1-\rho_{u, u^{\prime}}^{2}}} e^{-\frac{1}{2\left(1-\rho_{u, u^{\prime}}^{2}\right.}\left[\frac{\left(u_{i}-\mu_{u}\right)^{2}}{\sigma_{u}^{u}}-2 \rho_{u, u^{\prime}} \frac{\left(u_{i}-\mu_{u}\right)\left(u_{i}^{\prime}-\mu_{u^{\prime}}\right)}{\sigma_{u} \sigma_{u^{\prime}}}+\frac{\left(u_{i}^{\prime}-\mu_{u^{\prime}}\right)^{2}}{\sigma_{u^{\prime}}^{\prime}}\right]}
\end{aligned}
$$

where $u$ and $u^{\prime}$ represent the transverse phase space coordinates in either $x$ or $y$ direction, $z_{i}=z\left(u_{i}, u_{i}^{\prime}\right)$ is the real bivariate probability density value at $\left(u_{i}, u_{i}^{\prime}\right) . \mu_{u}$

and $\mu_{u^{\prime}}$ are the mean values of $u$ and $u^{\prime}, \sigma_{u}$ and $\sigma_{u^{\prime}}$ are the standard deviation of $u$ and $u^{\prime}$, and $\rho_{u, u^{\prime}}$ is the correlation between $u$ and $u^{\prime}$. Therefore, in this case, $\boldsymbol{\alpha}=\left(\mu_{u}, \mu_{u^{\prime}}, \sigma_{u}, \sigma_{u^{\prime}}, \rho_{u, u^{\prime}}\right)$. In the latter part of this section, we will $\rho_{u, u^{\prime}}$ as $\rho$ to be brief. The Jacobian matrix is thus

$$
\left(\boldsymbol{J}_{\boldsymbol{r}}\right)_{i, j}=\frac{\partial r_{i}}{\partial \alpha_{j}}, \quad i=1, \ldots, n ; \quad j=1, \ldots, 5
$$


The detailed matrix elements are,

$$
\begin{aligned}
& \frac{\partial r_{i}}{\partial \alpha_{1}}=\frac{\partial r_{i}}{\partial \mu_{u}}=-F \cdot\left[-\frac{1}{\left(1-\rho^{2}\right)}\left(-\frac{\left(u_{i}-\mu_{u}\right)}{\sigma_{u}^{2}}+\frac{\rho\left(u_{i}^{\prime}-\mu_{u^{\prime}}\right)}{\sigma_{u} \sigma_{u^{\prime}}}\right)\right] \\
& \frac{\partial r_{i}}{\partial \alpha_{2}}=\frac{\partial r_{i}}{\partial \mu_{u^{\prime}}}=-F \cdot\left[-\frac{1}{\left(1-\rho^{2}\right)}\left(-\frac{\left(u_{i}^{\prime}-\mu_{u^{\prime}}\right)}{\sigma_{u^{\prime}}^{2}}+\frac{\rho\left(u_{i}-\mu_{u}\right)}{\sigma_{u} \sigma_{u^{\prime}}}\right)\right] \\
& \frac{\partial r_{i}}{\partial \alpha_{3}}=\frac{\partial r_{i}}{\partial \sigma_{u}}=-F \cdot\left(-\frac{1}{\sigma_{u}}-\frac{1}{1-\rho^{2}}\left(\frac{\left(u_{i}-\mu_{u}\right)^{2}}{\sigma_{u}^{3}}+\frac{\rho\left(u_{i}-\mu_{u}\right)\left(u_{i}^{\prime}-\mu_{u^{\prime}}\right)}{\sigma_{u^{\prime}} \sigma_{u}^{2}}\right)\right) \\
& \frac{\partial r_{i}}{\partial \alpha_{4}}=\frac{\partial r_{i}}{\partial \sigma_{u^{\prime}}}=-F \cdot\left(-\frac{1}{\sigma_{u^{\prime}}}-\frac{1}{1-\rho^{2}}\left(\frac{\left(u_{i}^{\prime}-\mu_{u^{\prime}}\right)^{2}}{\sigma_{u^{\prime}}^{3}}+\frac{\rho\left(u_{i}-\mu_{u}\right)\left(u_{i}^{\prime}-\mu_{u^{\prime}}\right)}{\sigma_{u} \sigma_{u^{\prime}}^{2}}\right)\right) \\
& \frac{\partial r_{i}}{\partial \alpha_{5}}=\frac{\partial r_{i}}{\partial \rho}=-F \cdot\left(\frac{\rho}{1-\rho^{2}}-\frac{\rho}{\left(1-\rho^{2}\right)^{2}}\left(\frac{\left(u_{i}-\mu_{u}\right)^{2}}{\sigma_{u}^{2}}-2 \rho_{u, u^{\prime}} \frac{\left(u_{i}-\mu_{u}\right)\left(u_{i}^{\prime}-\mu_{u^{\prime}}\right)}{\sigma_{u} \sigma_{u^{\prime}}}+\frac{\left(u_{i}^{\prime}-\mu_{u^{\prime}}\right)^{2}}{\sigma_{u^{\prime}}^{2}}\right)\right. \\
&\left.+\frac{1}{1-\rho^{2}} \frac{\left(u_{i}-\mu_{u}\right)\left(u_{i}^{\prime}-\mu_{u^{\prime}}\right)}{\sigma_{u} \sigma_{u^{\prime}}}\right)
\end{aligned}
$$

The initial guess, $\boldsymbol{\alpha}^{(0)}$ is chosen to be

$$
\boldsymbol{\alpha}^{(0)}=\left(\overline{\boldsymbol{u}}, \overline{\boldsymbol{u}^{\prime}}, \sigma_{\boldsymbol{u}}, \sigma_{\boldsymbol{u}^{\prime}}, \rho_{\boldsymbol{u}, \boldsymbol{u}^{\prime}}\right)
$$

where $\rho_{\boldsymbol{u}, \boldsymbol{u}^{\prime}}$ is the correlation coefficient. The iterative procedure (A.3) is then applied until the aborting criterion

$$
\operatorname{std}\left(\frac{\Delta \boldsymbol{\alpha}}{\boldsymbol{\alpha}}\right) \leq 1 \times 10^{-5}
$$

is satisfied.

\section{A.2 Simulated Binary Crossover Operator}

The Simulated Binary Crossover (SBX) operator was developed by Deb and Agrawal

in 1995 [47]. The method of generating two offspring values $\alpha_{i}^{(1, t+1)}$ and $\alpha_{i}^{(2, t+1)}$ from two parent values $\alpha_{i}^{(1, t)}$ and $\alpha_{i}^{(2, t)}$ where $t$ is the generation number is described as 
follows. First, a spread factor $\beta_{i}$ is calculated based on a random number $u_{i} \in[0,1)$ such that

$$
\beta_{i}= \begin{cases}\left(2 u_{i}\right)^{\frac{1}{\eta_{c}+1}}, & \text { if } \mathrm{u}_{\mathrm{i}} \leq 0.5 \\ \left(\frac{1}{2\left(1-u_{i}\right)}\right)^{\frac{1}{\eta_{c}+1}}, & \text { otherwise }\end{cases}
$$

where $\eta_{c}$ is called the distribution index, which is any non-negative real number. A larger value of $\eta_{c}$ gives a higher probability of creating genes closer to the parents, and a smaller value gives a more wide spread offspring gene over the parameter space (see Figure A.1 for an example of offspring values from parent values=2 and 3).

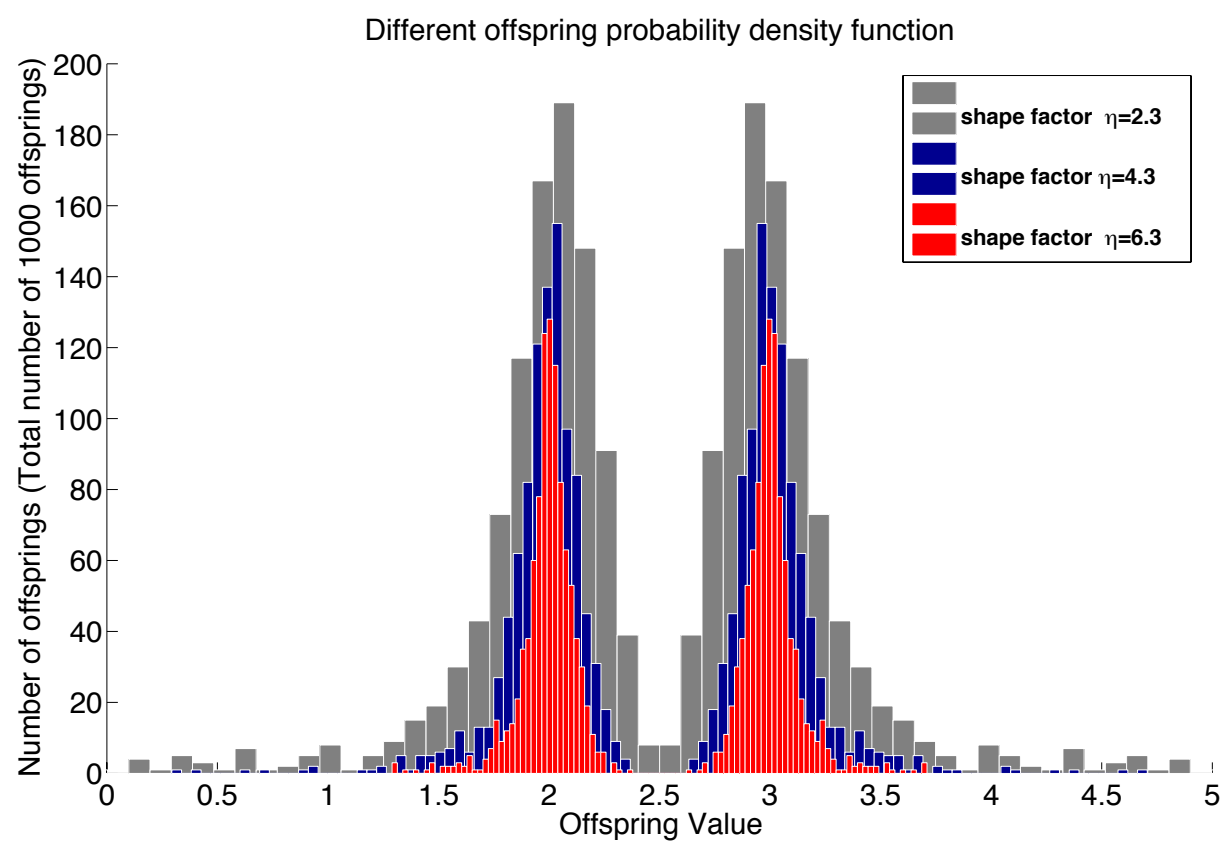

Figure A.1: Distribution of offspring values from two parents 2 and 3 with different $\eta_{c}$. The child generation is closer to the parents with greater $\eta_{c}$. 
After obtaining $\beta_{i}$ from the above formula, the offspring are calculated with

$$
\begin{aligned}
& \alpha_{i}^{(1, t+1)}=0.5\left[\left(1+\beta_{i}\right) \alpha_{i}^{(1, t)}+\left(1-\beta_{i}\right) \alpha_{i}^{(2, t)}\right] \\
& \alpha_{i}^{(2, t+1)}=0.5\left[\left(1-\beta_{i}\right) \alpha_{i}^{(1, t)}+\left(1+\beta_{i}\right) \alpha_{i}^{(2, t)}\right]
\end{aligned}
$$

This procedure is repeated for every $\alpha_{i}$ to form the $(t+1)$ th generation of individuals

$\boldsymbol{\alpha}^{(1, t+1)}, \ldots, \boldsymbol{\alpha}^{(N, t+1)}$ from the selected parents in the $t$ th generation of $\boldsymbol{\alpha}^{(1, t)}, \ldots, \boldsymbol{\alpha}^{(N, t)}$

\section{A.3 Simulated Annealing Algorithm}

The Simulated Annealing (SA) algorithm mimics the process of slowly cooling a metal to reduce the number of defects in it. It is a generic metaheuristic method to find a good approximation to the global optimum in a relatively short period of time. In this algorithm, neighborhood solutions of the current best solution are accepted with a probability controlled by a temperature parameter $T$, if the neighborhood solutions are worse than the current best. At the beginning stage of the algorithm, the $T$ is large enough to have very high probability of accepting poorer solutions, which guarantees a global search for the possible global optimum. As the temperature decreases, the searches are gradually confined in a small neighborhood of the current best until $T$ drops to the critical value set by the algorithm.

Since the probability is determined by the temperature following an exponential function $\exp (-\Delta f / T)$, where $\Delta f$ is the difference between the objective fitness value of the worse neighborhood solution and the current best, choosing appropriate values of $T$ and $\Delta T$ is thus critical for the algorithm to find the global optimum. Generally speaking, choosing a $T$ value that is close to the mean value of $f$ is preferred. This requires a basic understanding of the problem to be examined. The pseudo code of the SA algorithm is shown in Figure A.1. 


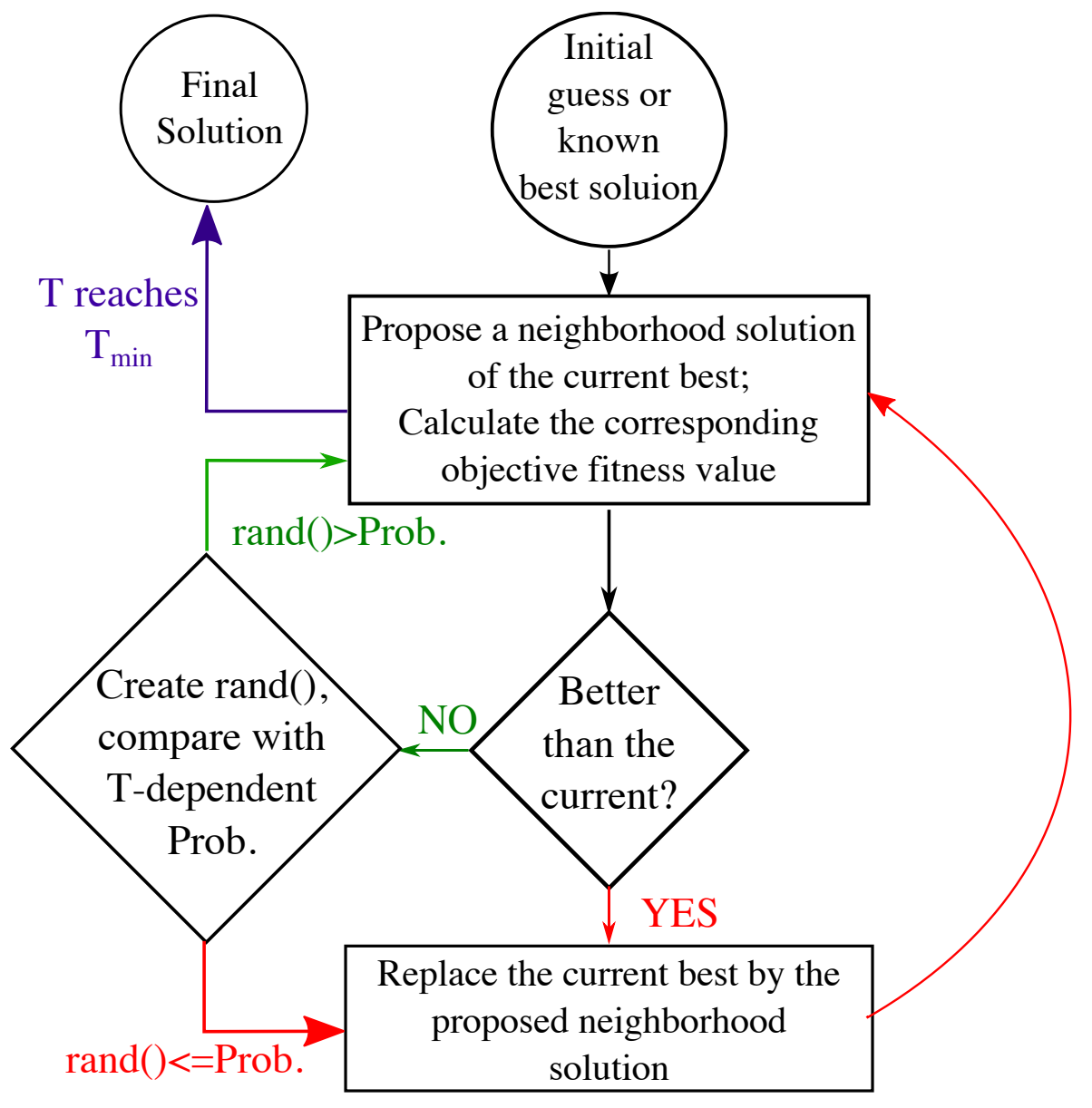

Table A.1: The SA algorithm flowchart. 


\section{A.4 Converting MAD-X outputs to G4BL input}

In this section the parsing algorithm that is used in converting the MAD-X outputs to G4BL input is discussed. The pseudo-code of the Python conversion code "mad2g4bl.py" is shown as a demonstration of the algorithm.

\section{A.4.1 Requirements and Strategies}

The conversion is done sequentially from the very first element of the beamline to the last. Because MAD-X uses different conventions from G4BL in defining the magnets, a parsing is needed. In MAD-X, the strength of each magnet is the relative strength $K_{n}$ defined by $K_{n}=B_{n} / B \rho$, where $B \rho$ is the magnetic rigidity of the reference particle. The information of the reference particle can be obtained by using the "twiss" command in MAD-X. However, the sequence of the beamline in the "twiss" output doesn't contain the physical properties of the magnets. Thus, another file that contains the detailed magnet information is needed, which can be obtained by using the "save,sequence" command in MAD-X. The challenge from this step is to substitute each variable used in describing the magnet properties by its value. For example, a quadrupole can be defined in the following format,

qExample: quadrupole, $1:=1$ Example ,k1:= -1 kExample;

where lExample and kExample are the variables defined before the definition of the quadrupole, by

lExmaple=0.3;

kExample:=kExamplePrime $* 0.5$;

where the $k$ ExamplePrime can be another variable defined earlier. The nesting usage of variable definitions can be multi-layer. Fortunately, variables can only be used after 
their values have been defined in MAD-X, which is also true in the "save, sequence" output. Moreover, the definition of each variable can be recognized immediately through its "varName $=(:=)$ varValueExp". The "mad2g4bl.py" takes advantage of this feature and calculates each sequentially defined variable in advance before starting to parse the magnets. Variables in MAD-X are stored as real variables in Python by using "exec". The positions of the magnets in the saved sequence are counted by their centers. This is also taken into consideration when calculating the drift space lengths between magnets since they are not saved in the sequence. Another feature is added in the "mad2g4bl.py" code that converts the markers in MAD-X to "zntuples" in G4BL. This forces G4BL to record the beam information exactly at the marked location.

The code takes the user's input, in which the particle name, the default beam pipe aperture, and some other options are defined, and starts the conversion. The default input beam can be either a Gaussian beam (built-in to of G4BL's beam command), or left blank for exterior beam files. A "particlefilter" is placed at the very beginning of the beamline, which is reserved to control the tracking for a ring lattice. In other words, the beam can be killed by the particlefilter after circulating in the ring for a certain amount of time. When any of the magnets have a defined aperture size, the beam pipe radius is changed accordingly. At the end of the beamline, a "zntuple" is used to record the beam for the last time.

\section{A.4.2 Pseudo-code of "mad2g4bl.py"}

A short pseudo-code below shows the skeleton of the algorithm.

$$
\begin{aligned}
& \text { particle="particleName" \# User input } \\
& \text { apert=" apertureSize" \#User input }
\end{aligned}
$$




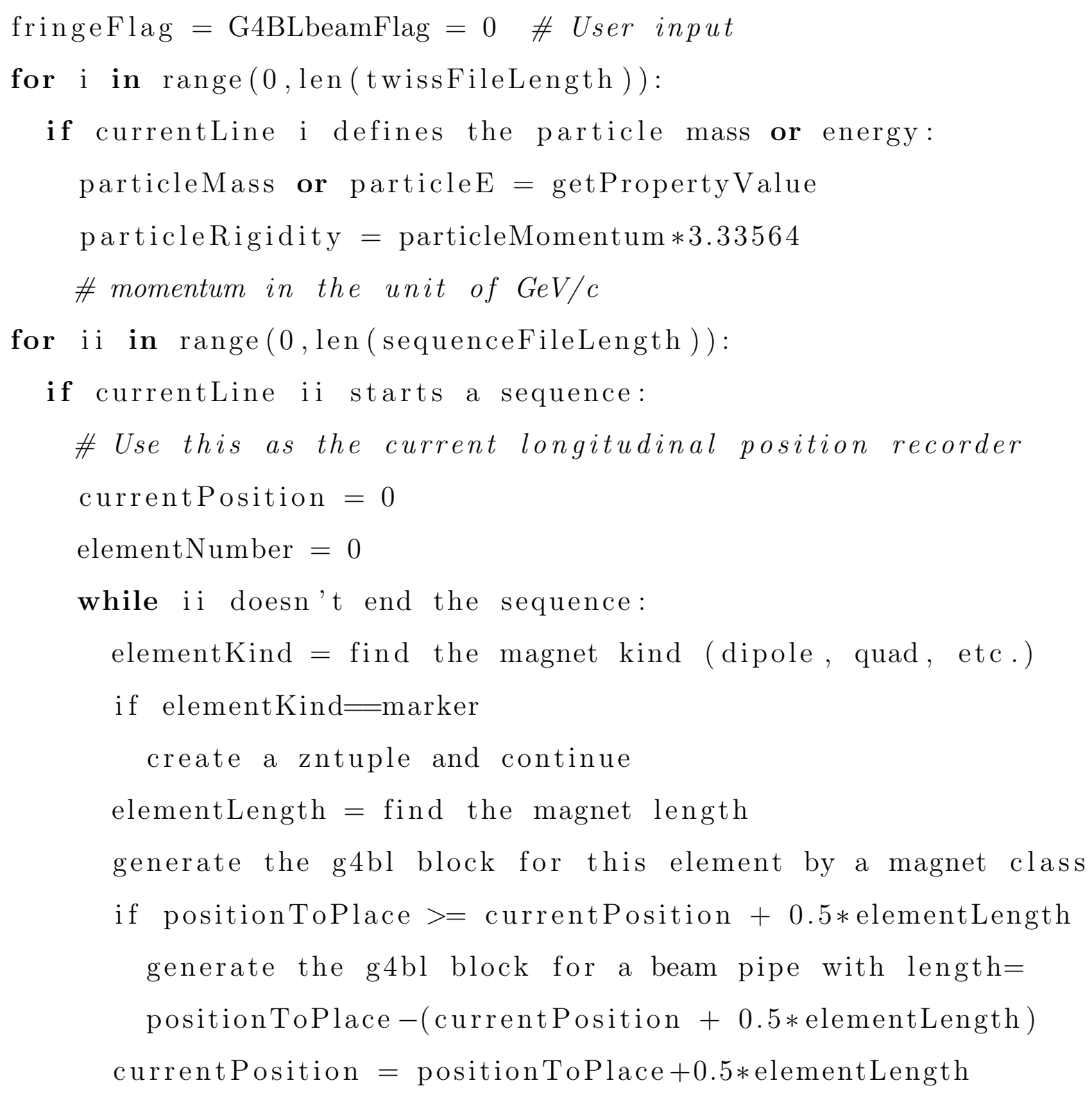

\section{A.5 Definition of the MADX pole-face rotation angles}

There are two types of bending magnets in MADX - SBEND and RBEND, which are the sector bending magnet and the rectangular bending magnet, respectively. The 
pole faces of a SBEND, without pole-face rotation angles, intersect perpendicularly with the ideal orbit of the reference particle, while the RBEND, without pole-face rotation angles, have parallel pole-faces and intersect with the ideal orbit with the same angle at the entrance and exit. Examples of an SBEND and a RBEND without pole-face rotation angles are drawn in blue in Figure A.2. Since the actual pole-faces of the bending magnet can form arbitrary angles from $-90^{\circ}$ to $90^{\circ}$ with respect to the ideal orbit, MADX then defines a convention to describe the shapes of the polefaces and the rotation angles, which are demonstrated by examples in dashed black in Figure A.2.

The rotation angles provide edge focusing or defocusing effects, thus need to be evaluated carefully for the OCS design (Section 3.3). Taking the entrance pole-face rotation angle as an example, the effective transfer matrices of the entrance pole-face can be written as

$$
\mathbf{M}_{\mathbf{e n t}}=\left(\begin{array}{cccc}
1 & 0 & 0 & 0 \\
\frac{\tan \mathrm{e}_{1}}{\rho} & 1 & 0 & 0 \\
0 & 0 & 1 & 0 \\
0 & 0 & -\frac{\tan \mathrm{e}_{1}}{\rho} & 1
\end{array}\right) \text {, corresponding to }\left(\begin{array}{c}
x \\
x^{\prime} \\
y \\
y^{\prime}
\end{array}\right)
$$

where $\rho$ is the bending radius and the small angle approximation that $\mathrm{e}_{1} \approx 0$ has been applied. A positive $e_{1}$ transversely defocuses and vertically focuses the beam, where the vertical focusing term vanishes when the fringe field is not considered.

In contrast, the current version of G4BL does not support bending magnets with pole-face rotation angles. 

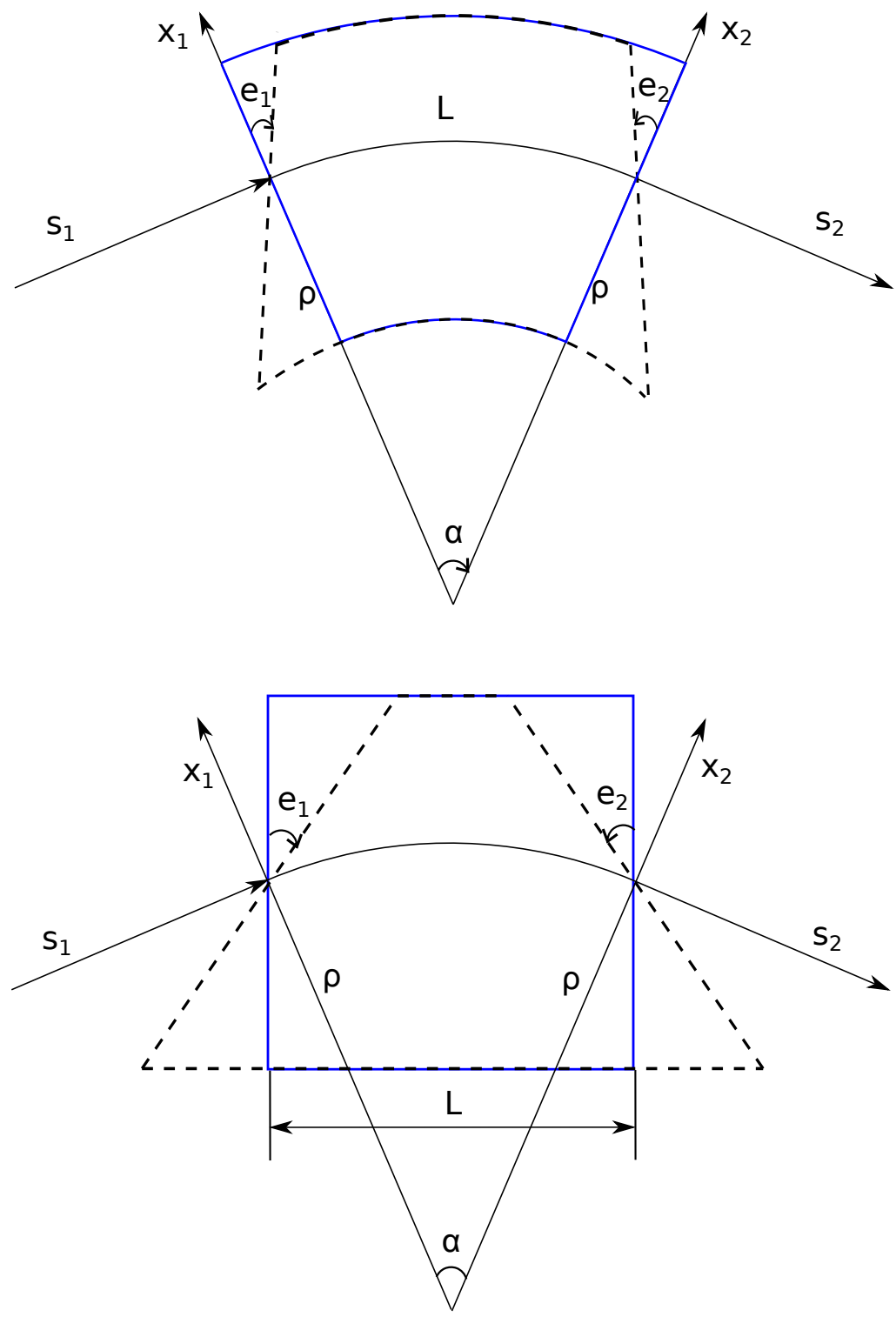

Figure A.2: Examples of bending magnets with (in solid and blue) and without (in dashed and black) pole-face rotation angles. The upper and lower figures represent the SBEND and RBEND configurations, respectively. 


\section{BIBLIOGRAPHY}

[1] C. Albright et al., "The neutrino factory and beta beam experiments and development," arXiv preprint physics/0411123, 2004.

[2] J. S. Berg et al., "Cost-effective design for a neutrino factory," Phys. Rev. ST Accel. Beams, vol. 9, p. 011001, Jan 2006.

[3] D. Adey et al., "nuSTORM and a Path to a Muon Collider." submitted to Annual Reviews,.

[4] S. Lee, "Accelerator physics," 1999.

[5] "Precise determination of the mass of the Higgs boson and studies of the compatibility of its couplings with the standard model," Tech. Rep. CMS-PAS-HIG14-009, CERN, Geneva, 2014.

[6] G. Aad et al., "Measurement of the higgs boson mass from the $H \rightarrow \gamma \gamma$ and $H \rightarrow Z Z^{*} \rightarrow 4 \ell$ channels in $p p$ collisions at center-of-mass energies of 7 and 8 tev with the atlas detector," Phys. Rev. D, vol. 90, p. 052004, Sep 2014.

[7] S. Geer, "Muon colliders and neutrino factories," ARXIV:1202.2140, 2010.

[8] C. D. Tunnell, "Sensitivity to electronvolt-scale sterile neutrinos at a 3.8-GeV/c muon decay ring," 2013. 
[9] C. W. Kim and A. Pevsner, Neutrinos In Physics and Astrophysics. 1993.

[10] D. Forero, M. Tortola, and J. Valle, "Global status of neutrino oscillation parameters after Neutrino-2012," Phys.Rev., vol. D86, p. 073012, 2012.

[11] A. Sousa, "First minos+ data and new results from minos." Proceedings of Neutrino 2014, 2014.

[12] Y. Ashie et al., "Measurement of atmospheric neutrino oscillation parameters by super-kamiokande i," Phys. Rev. D, vol. 71, p. 112005, Jun 2005.

[13] S. Geer, "Neutrino beams from muon storage rings: Characteristics and physics potential," Phys.Rev., vol. D57, pp. 6989-6997, 1998.

[14] P. Kyberd et al., "nuSTORM - Neutrinos from STORed Muons: Letter of Intent to the Fermilab Physics Advisory Committee," 2012.

[15] K. Abazajian, M. Acero, S. Agarwalla, A. Aguilar-Arevalo, C. Albright, et al., "Light Sterile Neutrinos: A White Paper," 2012.

[16] P. Astier et al., "Prediction of neutrino fluxes in the NOMAD experiment," Nucl.Instrum.Meth., vol. A515, pp. 800-828, 2003.

[17] J. Flynn, "Magnetic horn focusing for a low-energy neutrino beam," in $C E R N / E$ F/BEAM 81-5, 1981.

[18] K. Kopp, "The numi neutrino beam at fermilab," in Fermilab-Conf-05-093-AD, 2005.

[19] V. Papadimitriou, R. Andrews, J. Hylen, T. Kobilarcik, A. Marchionni, et al., "Design of the LBNE Beamline," p. TUOAA02, 2014. 
[20] N. Mokhov, "Mars15."

[21] L. Deniau et al., "Mad project main web page." http://mad.web.cern.ch/ $\mathrm{mad} /, 2002-$ Now.

[22] L. Deniau, "MAD-X Progress and Future Plans," p. THAAI3, 2012.

[23] F. Schmidt, "Mad-x ptc integration," in Particle Accelerator Conference, 2005. PAC 2005. Proceedings of the, pp. 1272-1274, May 2005.

[24] P. K. Skowronski, F. Schmidt, and E. Forest, "Advances in mad-x using ptc," in Particle Accelerator Conference, 200\%. PAC. IEEE, pp. 3381-3383, June 2007.

[25] P. K. Skowronski, F. Schmidt, et al., "New developments of mad-x using ptc," in Proceedings of ICAP 2006, 2006.

[26] G. Collaboration, "Geant4 main website." http://geant4.cern.ch/.

[27] A. Sato et al., "G4beamline simulation for the comet solenoid channel," in Proceedings of IPAC'10, Kyoto, Japan, 2010.

[28] T. J. Roberts et al., "G4beamline particle tracking in matter-dominated beam lines," in Proceedings of EPAC08, Genoa, Japan, 2008.

[29] USING GEANT4-BASED TOOLS TO SIMULATE A PROTON EXTRACTION AND TRANSFER LINE, 2009.

[30] K. Beard and M. Inc., "g4beamline and e05115." JLABTN0875.

[31] L. Dalcin, "mpi4py main web page." http://mpi4py.scipy.org/.

[32] "Numpy main web page." http://www. numpy.org/. 
[33] N. E. R. S. C. Center, "http://www.nersc.gov/."

[34] J. J. Back, C. Densham, R. Edgecock, and G. Prior, "Particle production and energy deposition studies for the Neutrino Factory target station," Phys.Rev.ST Accel.Beams, vol. 16, no. 2, p. 021001, 2013.

[35] D. M. Kaplan, "Neutrino Factory R\&D Efforts," 2013.

[36] D. M. Kaplan, "Muon Cooling, Muon Colliders, and the MICE Experiment," 2013.

[37] D. Neuffer, "Design considerations for a muon storage ring," 1980. Telmark Conference on Neutrino Mass, Barger and Cline eds., Telmark, Wisconsin.

[38] S. van der Meer, "A directive device for charged particles and its use in an enhanced neutrino beam," CERN-61-0\%, 1961.

[39] A. Abramov, N. Galyaev, V. Garkusha, J. Hylen, F. Novoskoltsev, A. Ryabov, and V. Zarucheisky, "Beam optics and target conceptual designs for the numi project," Nuclear Instruments and Methods in Physics Research Section A: Accelerators, Spectrometers, Detectors and Associated Equipment, vol. 485, no. 3, pp. $209-227,2002$.

[40] A. Ichikawa, "Design concept of the magnetic horn system for the $\{\mathrm{T} 2 \mathrm{~K}\}$ neutrino beam," Nuclear Instruments and Methods in Physics Research Section A: Accelerators, Spectrometers, Detectors and Associated Equipment, vol. 690, no. 0, pp. $27-33,2012$.

[41] P. Adamson et al., "Search for the disappearance of muon antineutrinos in the NuMI neutrino beam," Phys.Rev., vol. D84, p. 071103, 2011. 
[42] M. Bishai, "Optimizing the Geometry of the Focusing Horn Magnets in the Long-Baseline Neutrino Experiment Beamline," LBNE-doc-6265-v1.

[43] K. Deb, A. Pratap, S. Agarwal, and T. Meyarivan, "A fast and elitist multiobjective genetic algorithm: Nsga-ii," Trans. Evol. Comp, vol. 6, pp. 182-197, Apr. 2002.

[44] Y. Luo, M. Bai, et al., "Off-momentum beta-beat correction in the RHIC proton run," 2012.

[45] J. Bengtsson, "The Sextupole Scheme for the Swiss Light Source (SLS): An Analytic Approach," 1997.

[46] M. Borland, "Advanced photon source, anl." http://www.aps.anl.gov/ Accelerator_Systems_Division/Accelerator_Operations_Physics/ software.shtml\#elegant.

[47] R. B. Agrawal, K. Deb, K. Deb, and R. B. Agrawal, "Simulated binary crossover for continuous search space," tech. rep., 1994. 
Ao Liu

Ph.D.

Curriculum Vitae

\section{PERSONAL INFORMATION}

Year Of Birth 1990

Email aoliu@fnal.gov, aoliu@indiana.edu

Website Www.frankliuao.com

\section{CURRENT RESEARCH INTEREST} Simulations, Novel Algorithms and Coding in Physics, Beam Dynamics, Novel Concepts in Beam Applications

\section{CURRENT POSITION}

Guest Scientist

Fermi National Accelerator Laboratory (Fermilab)

2015-Present

\section{EDUCATION}

Physics Ph.D.

2010-2015

Supervisors: Prof. Shyh-yuan Lee (IU), Dr. Alan Bross, Dr. David Neuffer (Fermilab)

Indiana University and Fermilab Joint Ph.D. Program

U.S. Particle Accelerator School

2011-2014

Classes taken:

Medical Applications of Accelerators, Pulsed Power Engineering, Microwave Measurements 
and Beam Instrumentation Lab, Fundamentals of Detector Physics, Control Theory with Applications to Accelerators and RF Systems, Vacuum Science and Technology for Accelerator Vacuum Systems, Cryogenic Engineering, Vibrational Aspects of Accelerators, Design of Room Temperature Magnets

All classes taken for credits with final grades $\geq \mathrm{A}$

B.S. of Physics

2006-2010

Lanzhou University, China

Degree authenticated by National Theoretical Physicists Base of China

\section{WORK EXPERIENCE}

\section{Research Associate}

2012-Present

Fermilab

Participated in the Fermilab Joint Ph.D Program and started to work on the neutrinos from STORed Muons (nuSTORM) project. Designed the pion beamline and the muon decay ring for nuSTORM. Realized the stochastic injection of pions in a muon decay ring. Studied the beam dynamics and analyze the particles in the nuSTORM beamline using tracking programs. Developed parsing codes to convert Beamline simulation input to multiple platforms. Performed the horn optimization studies for nuSTORM using Python Message Passing Interface (MPI) based Genetic Algorithm.

\section{Research Associate}

2010-2012

Indiana University Bloomington

Worked on the basics of accelerator physics. Worked on the beamline design examples like a Compact Medical Synchrotron (CMS), MATLAB applications in Independent Component Analysis(ICA), beam commissioning and electronics improvement for ALPHA electron storage ring facility of Indiana University. Performed the data measurement and analysis for ALPHA electron facility on campus.

\section{Associate Instructor}

Indiana University Bloomington

Served as the AI for P109 - Basic Acoustic Lab Course. Received top evaluation from the students because of my novelty in teaching.

\section{Research Associate}

2009-2010

Peking University, Intern

Completed B.S graduation thesis on simulations of Separated Function Radio Frequency Quadrupole (SFRFQ) in Peking University. Studied and applied 3D electromagnetic field simulation and 
data analysis software such as CST Microwave Studio and MATLAB.

Undergraduate Intern

2009

Institute of Modern Physics, CAS

\section{PUBLICATIONS}

A. Liu, A. Bross et al. "nuSTORM horn optimization study", Proceedings of IPAC2014, Dresden, Germany, 2014

A. Liu, A. Bross et al. "nuSTORM ring design update", Proceedings of IPAC2014, Dresden, Germany, 2014

A. Liu, A. Bross et al. " $\nu$ STORM FACILITY DESIGN AND SIMULATION", Proceedings of IPAC2013, Shanghai, China, 2013.

A. Bross, S. Geer, A. Liu, et al. "DESIGN CONCEPT FOR NU-STORM: AN INITIAL VERY LOW-ENERGY NEUTRINO FACTORY", Proceedings of IPAC2012, New Orleans, Louisiana, USA, 2012.

\section{CONTRIBUTED AND INVITED TALKS}

- Contributed talk at the 5th High Power Targetery Workshop, Fermilab, Batavia, U.S.A

- Invited talk at the 2014 MAP spring meeting, Fermilab, Batavia, U.S.A

- Contributed Talk at iPAC2013 (international Particle Accelerator Conference 2013), Shanghai, China

HONORS \& AWARDS

iPAC and NA'PAC Student Grants

2013-2014

iPAC and NA'PAC Locations 
Two consecutive years of student grants from iPAC and NA'PAC organizing committee, combined with funding support from APS and IEEE.

USPAS Student Grants

2011-Present

USPAS Locations

Student grants from USPAS (United States Particle Accelerator School) organizing committee.

INSS2013 Student Grants

2013

Beijing, China

Student grant from INSS (International Neutrino Summer School) organizing committee.

Chun-Tsung Scholar

2009

Lanzhou University

Funded by Nobel Physics Prize winner Tsung-Dao Lee and his wife.

JianxianSiqi Model

2009

Lanzhou University

Prize for undergraduate students who set up good examples for schoolmates

Outstanding League Cadre

2008

Lanzhou University

Award for cadres in the League who have done an outstanding job in serving the committee

Scholarship for Three Years

2006-2009

Lanzhou University

\section{SKILLS}

Coding Skills Python, bash, $\mathrm{C}++$, MATLAB, HTML

Software

G4Beamline (Geant4-Based Monte Carlo Simulation Software), MATLAB, MADX, LTEX, CST Microwave Studio, Coda, Photoshop CS6, MS Office, InkScape

Hardware

BPMs, Oscilloscopes, Linac Operations, Circuit Boards

Languages

Mandarin, English

\section{PERSONAL}


I am an enthusiastic person, with a wide range of interest and a very creative mind. In my spare time, I like doing sports, such as working out, fishing, table tennis, badminton, etc. I'm also a fan of good music and High-definition headphones, Chinese poetry, movies, and much more. 